\title{
Stochastic Optimization in Green Wireless Networks and Smart Grids
}

by

Shengrong Bu, B. Eng., M. Eng. (Research)

\author{
A thesis submitted to the \\ Faculty of Graduate and Postdoctoral Affairs \\ in partial fulfillment of the requirements for the degree of
}

Doctor of Philosophy in Electrical and Computer Engineering

Ottawa-Carleton Institute for Electrical and Computer Engineering

Department of Systems and Computer Engineering

Carleton University

Ottawa, Ontario

April 2012

(C) Copyright

Shengrong $\mathrm{Bu}, 2012$ 
Library and Archives

Canada

Published Heritage

Branch

395 Wellington Street

Ottawa ON K1A ON4

Canada
Bibliothèque et

Archives Canada

Direction du

Patrimoine de l'édition

395 , rue Wellington

Ottawa ON K1A ON4

Canada
Your file Votre référence

ISBN: 978-0-494-89324-1

Our file Notre référence

ISBN: $978-0-494-89324-1$

\section{NOTICE:}

The author has granted a nonexclusive license allowing Library and Archives Canada to reproduce, publish, archive, preserve, conserve, communicate to the public by telecommunication or on the Internet, loan, distrbute and sell theses worldwide, for commercial or noncommercial purposes, in microform, paper, electronic and/or any other formats.

The author retains copyright ownership and moral rights in this thesis. Neither the thesis nor substantial extracts from it may be printed or otherwise reproduced without the author's permission.
AVIS:

L'auteur a accordé une licence non exclusive permettant à la Bibliothèque et Archives Canada de reproduire, publier, archiver, sauvegarder, conserver, transmettre au public par télécommunication ou par l'Internet, prêter, distribuer et vendre des thèses partout dans le monde, à des fins commerciales ou autres, sur support microforme, papier, électronique et/ou autres formats.

L'auteur conserve la propriété du droit d'auteur et des droits moraux qui protege cette thèse. $\mathrm{Ni}$ la thèse ni des extraits substantiels de celle-ci ne doivent être imprimés ou autrement reproduits sans son autorisation.
In compliance with the Canadian Privacy Act some supporting forms may have been removed from this thesis.

While these forms may be included in the document page count, their removal does not represent any loss of content from the thesis.
Conformément à la loi canadienne sur la protection de la vie privée, quelques formulaires secondaires ont été enlevés de cette thèse.

Bien que ces formulaires aient inclus dans la pagination, il n'y aura aucun contenu manquant. 
The undersigned hereby recommends to the Faculty of Graduate and Postdoctoral Affairs acceptance of the thesis

\section{Stochastic Optimization in Green Wireless Networks and Smart Grids}

submitted by Shengrong Bu, B. Eng., M. Eng. (Research)

in partial fulfillment of the requirements for the degree of

Doctor of Philosophy in Electrical and Computer Engineering

External Examiner, Professor Yi Qian, Department of Computer and Electronics Engineering, University of Nebraska-Lincoln

Thesis Supervisor, Professor F. Richard Yu

Thesis Supervisor, Professor Peter X. Liu

Chair, Professor Howard Schwartz,

Department of Systems and Computer Engineering

Ottawa-Carleton Institute for Electrical and Computer Engineering

Department of Systems and Computer Engineering

Carleton University

April, 2012 


\section{Abstract}

Information and communications technologies (ICT) have a very significant role to play in addressing climate change globally and facilitating low carbon development. The role of ICT includes not only the emission reduction and energy savings in ICT products and services, but also enabling low carbon emissions in other industries, such as electric power smart grids. This thesis studies stochastic schemes in green wireless cellular networks, mobile ad-hoc networks (MANETs) and smart grids to make them more efficient, effective, and reliable. We present a distributed scheme combining continuous user authentication and intrusion detection in high security green MANETs, taking security posture and energy efficiency into account. A structural results method is proposed to lower the computational complexity of this scheme. Moreover, in smart grids, highly intermittent renewable energy sources and stochastic power demand loads make the balance question complicated to solve. We propose a distributed stochastic unit commitment scheduling scheme and a novel gametheoretical decision-making scheme for electricity retailers in smart grids. Partially observable Markov decision process (POMDP) multi-armed bandit models are used to analyze and solve the above decision problems under environmental uncertainties. Finally, we consider not only energy-efficient communications but also the dynamics of smart grids in operating green wireless cellular networks powered by smart grids, which is modeled as a Stackelberg game. Simulation results are presented to show the effectiveness of the proposed schemes. 


\section{Acknowledgments}

I would like extend my heartfelt thanks and sincere gratitude to my co-supervisors,

Professors F. Richard Yu and Peter X. Liu, for their guidance while I was carrying out this research. They provided a constant source of support and encouragement when I was troubled by uncertainties. This thesis would not have been possible without their invaluable support.

Special thanks are due to the committee members for the detailed reviews and constructive comments, which have helped to improve the quality of this thesis.

An important thank you must go to my co-authors and research collaborators: Helen Tang and Peter Mason from DRDC, Yegui Cai from my group, Peng Zhang from University of Connecticut have all enriched my work and my understanding. Also thanks to Hussein Al-Zubaidy for his advice on research and on life in general.

Ian Scrimger has been understanding and helpful throughout my $\mathrm{PhD}$ study.

Finally, I am especially grateful to my family and my relatives. My father and my mother have been wonderful to me, and have helped me achieve what I have today. 


\section{Table of Contents}

$\begin{array}{ll}\text { Abstract } & \text { iii }\end{array}$

Acknowledgments $\quad$ iv

Table of Contents $\quad$ v

List of Tables $\quad x$

List of Figures $\quad$ xi

List of Acronyms $\quad$ Xv

1 Introduction 1

1.1 Problem Statement . . . . . . . . . . . . . . . . . . 2

1.2 Contributions and Publications . . . . . . . . . . . . 6

1.3 Thesis Organization . . . . . . . . . . . . . . . 10

2 Overview of Green Wireless Networks and Smart Grids 11

2.1 High Security Green MANETs . . . . . . . . . . . . . . . . . . 12

2.1.1 Biometric-Based Continuous User Authentication . . . . . . . 12

2.1.2 Intrusion Detection Systems . . . . . . . . . . . . . 14

2.1.3 Combined Continuous User Authentication and Intrusion Detection ....................... 16

2.2 Smart Grids . . . . . . . . . . . . . . . . . 16

2.2.1 Unit Commitment . . . . . . . . . . . . . . . 17

2.2.2 Demand-Side Management and Supporting Standards in Smart Grids . . . . . . . . . . . . . . . . . 19

2.2.2.1 Demand-Side Management . . . . . . . . 19 
2.2.2.2 Smart Grid Standards Supporting Demand-Side Management . . . . . . . . . . . . . 2 22

2.2.3 The Decision-Making of Electricity Retailers . . . . . . . . 23

2.2.4 Smart Grid Communication Infrastructures . . . . . . . 25

2.2.4.1 Network Architecture for Smart Grids . . . . . . 25

2.2.4.2 Major Requirements for Smart Grid Communication Infrastructures . . . . . . . . . 27

2.3 Green Wireless Cellular Networks . . . . . . . . . . . . . . . . 29

2.3.1 Energy-Efficient Solutions _ . . . . . . . . . . . . . . 29

2.3.1.1 Renewable Energy Resources . . . . . . . . . . . 30

2.3.1.2 Energy-Aware Cooperative BS Power Management . 30

2.3.1.3 Cognitive Heterogeneous Networks . . . . . . . . 31

2.3.2 Key Trade-offs in the Wireless Cellular Networks . . . . . . 33

2.4 Conclusion . . . . . . . . . . . . . . . . . . . . 34

3 Distributed Combined User Authentication and Intrusion Detection in High Security Green Mobile Ad-Hoc Networks $\quad 36$

3.1 Introduction . . . . . . . . . . . . . . 36

3.2 Distributed Combined User Authentication and Intrusion Detection with Data Fusion . . . . . . . . . . . . . . . . . 38

3.2 .1 System Model . . . . . . . . . . . . . . . . 38

3.2.2 Data Fusion of Biometric Sensors and IDSs . . . . . . . . . . 43

3.2.3 Formulation of the Distributed User Authentication and Intrusion Detection Scheduling Problem . . . . . . . . . 47

3.2.3.1 Information State Formulation . . . . . . . . 48

3.2.3.2 Distributed Scheduling Process . . . . . . . . . . 49

3.2.3.3 Discussion of Computational Complexity and Communication Overhead . . . . . . . . . . . 50

3.2.4 Simulation Results and Discussions . . . . . . . . . . . . 51

3.2.4.1 Performance Improvement . . . . . . . . . . . . . 54

3.2.4.2 Network Compromise Probability Improvement . . . 57

3.2.4.3 Network Lifetime Improvement and the Optimal Policy 58

3.3 Structural Results for Combined Continuous User Authentication and Intrusion Detection . . . . . . . . . . . . . . . 60

3.3.1 Monotone Gittins Index in the Structural Results Method . . 62 
3.3.2 Approximations to the Gittins Index $\ldots \ldots \ldots 6$

3.3.3 Computational Complexity and Communication Overhead . . 67

3.3.4 Simulation Results and Discussions . . . . . . . . . . 68

3.3.4.1 Computational Efficiency Comparison . . . . . . 70

3.3.4.2 Policies Derived from the Structural Results . . . . . 70

3.3.4.3 Performance Comparison . . . . . . . . . . 72

3.3.4.4 Network Lifetime Comparison . . . . . . . . . 77

3.4 Conclusion . . . . . . . . . . . . . . . . . . . . 78

\section{Stochastic Scheduling and Decision-Making Schemes in Smart} Grids

4.1 Introduction . . . . . . . . . . . . . . . . 80

4.2 Distributed Unit Commitment Scheduling . . . . . . . . . . . 82

4.2 .1 System Models . . . . . . . . . . . . . . . . . 83

4.2.1.1 Renewable Energy Model . . . . . . . . . . . 84

4.2.1.2 Power Generation Unit State Model . . . . . . . 86

4.2.1.3 Power Demand Model . . . . . . . . . . . . 87

4.2.1.4 Cost Model . . . . . . . . . . . . . . . . . . . 89

4.2.2 Formulation and Solutions to Stochastic Unit Scheduling Problem 91

4.2.2.1 Value Iteration Method . . . . . . . . . . . 91

4.2.2.2 Structural Results . . . . . . . . . . . . . . 93

4.2 .2 .3 Constraints . . . . . . . . . . . . . . 95

4.2.3 Simulation Results and Discussions _. . . . . . . . . . 96

4.2.3.1 Performance Improvement . . . . . . . . . . . . . . 98

4.2.3.2 Pollutant Emission Improvement _... . . . . . . . 101

4.2.3.3 Comparisons of Computational Efficiency and Performance . . . . . . . . . . . . . . 103

4.3 A Game-Theoretical Decision-Making Scheme for Electricity Retailers 104 4.3 .1 System Model . . . . . . . . . . . . . . . . . . . . . 104

4.3.1.1 Real-Time Electricity Demand Model for Customers 105

4.3.1.2 Electricity Supply Sources for Retailers . . . . . . . 107

4.3.1.3 A Four-stage Stackelberg Game Model . . . . . . . 107

4.3.2 Four-Stage Stackelberg Game Analysis . . . . . . . . . . 109

4.3.2.1 Real-Time Electricity Demand in Stage IV . . . . . . 109

4.3.2.2 Optimal Real-Time Pricing Strategy in Stage III _ . 110 
4.3.2.3 Optimal Electricity Procurement Strategy in Stage II 111

4.3.2.4 Optimal Procurement Strategy in Stage I . . . . . 114

4.3.2.5 The Retailer's Realization Profit . . . . . . . . . 118

4.3.3 Simulation Results and Discussions . . . . . . . . . . . 119

4.4 Conclusion . . . . . . . . . . . . . . . . . . . . 126

5 Dynamic Operations of Green Wireless Cellular Networks Powered by Smart Grids $\quad 129$

5.1 Introduction . . . . . . . . . . . . . . . . . . . 129

5.2 Dynamic Base Station Operations in Cellular Networks with CoMP

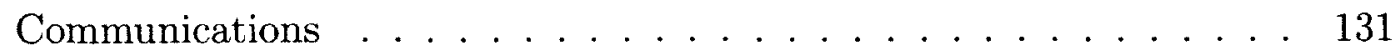

5.2 .1 System Models . . . . . . . . . . . . . . . . . . 132

5.2 .1 .1 CoMP Communications . . . . . . . . . . 132

5.2.1.2 Service Blocking Probability Model in the Cellular Network . . . . . . . . . . . . . . . . 134

5.2.1.3 Electricity Consumption Model for Base Stations . . 135

5.2 .2 Problem Formulation . . . . . . . . . . . . . . . . . 137

5.2.2.1 Cellular Network Level Game . . . . . . . . . . . 137

5.2.2.2 Smart Grid Level Game . . . . . . . . . . . . . 139

5.2.3 Analysis of the Proposed Two-Level Game . . . . . . . . . . . 140

5.2.3.1 Analysis of the Cellular Network Level Game . . . . 140

5.2.3.2 Analysis of the Smart Grid Level Game . . . . . . 142

5.2.3.3 Existence of Stackelberg Equilibrium for the Proposed Two-Level Game . . . . . . . . . . . . . . . . . 143

5.2.3.4 Uniqueness of the Stackelberg Equilibrium for the Proposed Two-Level Game . . . . . . . . . . . . 144

5.2.3.5 Model for Multiple Base Station Clusters . . . . . . . 147

5.2.4 Simulation Results and Discussions . . . . . . . . . . 148

5.3 Dynamic Energy-Efficient Resource Allocation in Cognitive Heterogeneous Wireless Networks . . . . . . . . . . . . . . . 155

5.3 .1 System Models . . . . . . . . . . . . . . . . 156

5.3.1.1 Wireless Heterogeneous Network Model . . . . . . 156

5.3.1.2 Electricity Consumption Model for Base Stations in Heterogeneous Networks . . . . . . . . . . . 158

5.3.1.3 Complete System Model . . . . . . . . . . . . . 158 
5.3 .2 Problem Formulation . . . . . . . . . . . . . . . . . 159

5.3.2.1 Smart Grid Level Game . . . . . . . . . . . . 160

5.3.2.2 MBS Level Game . . . . . . . . . . . . . . . . . 161

$5.3 .2 .3 \quad$ FBS Level Game . . . . . . . . . . . . . . . . 162

5.3.3 Analysis of the Proposed Three-Level Game . . . . . . . . . 163

5.3.3.1 Analysis of Power Allocation Game for FBSs . . . 163

5.3.3.2 Analysis of the MBS Level Game . . . . . . . . . 164

5.3.3.3 Analysis of the Homogeneous Bertrand Game for Electricity Retailers in Smart Grids . . . . . . . . 167

5.3.3.4 The Proposed Iteration Algorithm to Obtain the Stackelberg Equilibrium . . . . . . . . . . 171

5.3.4 Simulation Results and Discussions . . . . . . . . . . . . 173

5.4 Conclusion . . . . . . . . . . . . . . . . . . . . . . 181

6 Conclusions and Future Work $\quad 183$

6.1 Thesis Conclusions . . . . . . . . . . . . . . . . . . . 183

6.2 Future Work . . . . . . . . . . . . . . . . . . . . 185

$\begin{array}{ll}\text { List of References } & 187\end{array}$ 


\section{List of Tables}

3.1 The computation time in the centralized scheme and the proposed distributed scheme. . . . . . . . . . . . . . 71

3.2 Percentage of states not MLR comparable in the first 100 steps. . . . 72

4.1 The computation time of the two methods in the proposed distributed scheme. . . . . . . . . . . . . . . . . . . 103

4.2 Optimal price decision and maximum profit of the retailer in Stage III. 112

4.3 Optimal amount of electricity to procure and maximum profit in Stage II. . . . . . . . . . . . . . . . . . . . . 113

4.4 Optimal procurement strategy and maximum expected profit in Stage I.117

4.5 The retailer's and electricity customers' equilibrium behaviors. . . . . 118 


\section{List of Figures}

2.1 Smart electricity pricing models in smart grids. . . . . . . . . . . . . 20

2.2 Pricing schemes to develop RTP models in smart grids. . . . . . . . . 21

3.1 An example framework for a MANET with biosensors and IDSs. . . . 39

3.2 An example of Markov chain for a single node's state transition. . . . 41

3.3 Cost comparison among the proposed scheme with data fusion, the proposed scheme without data fusion and the existing scheme. . . . . 55

3.4 Information leakage comparison among three schemes. . . . . . . 55

3.5 Cost comparison among three schemes under different transition probabilities. ........................ 56

3.6 Information leakage comparison among three schemes under different transition probabilities. . . . . . . . . . . . . 57

3.7 Cost comparison among three schemes with varying nodes in the network. 58

3.8 Network compromise comparison among three schemes under different transition probabilities. . . . . . . . . . . . 59

3.9 Network lifetime comparison between the proposed scheme and the existing scheme under different energy transition probabilities. . . . 60

3.10 Network average lifetime with varying number of nodes. . . . . . . . . 61

3.11 An example of the policy derived from the proposed scheme without data fusion. (Action =1: The iris sensor is selected; Action $=2$ : The fingerprint sensor is selected; Action $=3$ : The IDS is selected) . . . .

3.12 An example policy derived from the structural results method (1: Iris; 2: Fingerprint; 3: IDS). . . . . . . . . . . . . . . 71

3.13 Average cost comparison of the centralized scheme, the random choice scheme, and the proposed distributed scheme. . . . . . . . . . . 74

3.14 Average information leakage comparison between the two methods. . 74

3.15 Average cost comparison between the two methods with different numbers of nodes. . . . . . . . . . . . . . 76 
3.16 Average information leakage comparison between civilian network and battlefield network with different numbers of nodes. . . . . . 76

3.17 Average cost comparison between the two methods with different state

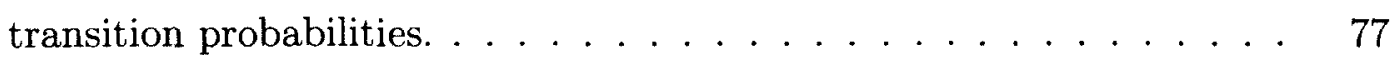

3.18 Average information leakage comparison between the two methods with different state transition probabilities. . . . . . . . . .

3.19 Network average lifetime comparison between civilian network and battlefield network with different numbers of network nodes. . . . . . . .

4.1 The relationships between supply side management, demand-side management and unit commitment. . . . . . . . . . . . 84

4.2 An example of 3-state Markov-modulated Poisson process (MMPP). . $\quad 87$

4.3 Total costs of the different schemes. . . . . . . . . . . . . . 99

4.4 Average costs of three schemes under different transition probabilities. 100

4.5 Average costs of three schemes with varying units in smart grids. . . 101

4.6 Total costs versus hours with various pollutant emission cost levels. . 102

$4.7 \mathrm{CO}_{2}$ emissions versus hours with various pollutant emission cost levels. 102

4.8 Total costs of the two methods in the proposed scheme. . . . . . . 104

4.9 A four-stage Stackelberg game proposed for modeling the interactions between a retailer and its customers. . . . . . . . . . . 108

4.10 The optimal amount of electricity procured from electricity source Option I with the changes of prices $C_{m}$ and $C_{g} \ldots \ldots \ldots \ldots$

4.11 The optimal amount of electricity procured from Option II with the changes of price $C_{g}$, price $C_{m}$, and realization factor $\beta$ when $C_{m}<\frac{C_{g}}{2}$.

4.12 The optimal price offered to customers with the changes of price $C_{g}$, price $C_{m}$, and realization factor $\beta$ when $C_{m}<\frac{C_{g}}{2} \ldots \ldots \ldots$

4.13 The highest expected profit with/without considering Option I with the change of price $C_{m}$ when $C_{m}<\frac{C_{g}}{2} \ldots \ldots \ldots \ldots \ldots$

4.14 The utility of customer $i$ with the change of realization factor $\beta$ when

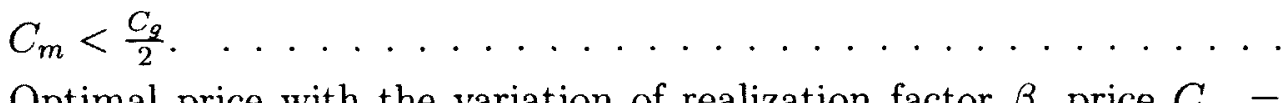

4.15 Optimal price with the variation of realization factor $\beta$, price $C_{m}=$

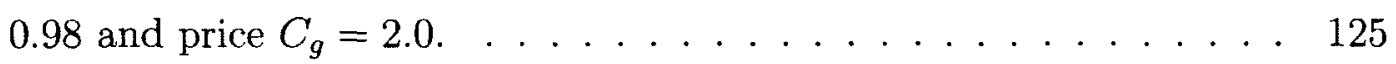

4.16 Optimal price with the variation of realization factor $\beta$, price $C_{m}=$ 0.70 and price $C_{g}=2.0 . \ldots \ldots \ldots \ldots$ 
4.17 Realization profit with the change of price $C_{g}$, price $C_{m}$ and realization factor $\beta$ with/without considering Option I. . . . . . . . 126

4.18 Realization utility of user $i$ with the change of realization factor with/without considering Option I. $X_{i}=3.0 \ldots \ldots \ldots \ldots \ldots$

5.1 A cellular network powered by smart grids. . . . . . . . . . . 132

5.2 Coverage extension and energy saving with CoMP. . . . . . . . 133

5.3 Service blocking probability and amount of electricity consumed as the combination of the base stations in a cluster varies, $\lambda=1.1$ and $\mu=1 / 180$. The numbers on the $x$ axis represent the indices of different elements of the cooperation set $\Theta$. In base station cooperation sets 2 , 3,4 , there is one active base station. In cooperation sets 5,6 and 7 , there are two active base stations. In cooperation set 8 , all three base stations are active.

5.4 Compare the traditional method with the proposed DBSOS with CoMP, in terms of electricity cost and $\mathrm{CO}_{2}$ emission as the traffic arrival rate $\lambda$ varies. . . . . . . . . . . . 150

5.5 Total operational expenditure of base stations. . . . . . . . . . 151

5.6 Utility of Retailer 1 and Retailer 2 as the price offered by Retailer 1 varies. . . . . . . . . . . . . . . . . . . . . . 152

5.7 Optimal prices offered by Retailer 1 and Retailer 2 as the pollutant parameter of Retailer $1\left(\alpha_{1}\right)$ varies. . . . . . . . . . . . 153

5.8 Optimal amount of electricity to procure from Retailer 1 and Retailer 2 as the pollutant parameter of Retailer $1\left(\alpha_{1}\right)$ varies. . . . . . . .

5.9 Utility of the optimal base station combination element $\Psi^{*}$ as the number of retailers varies. . . . . . . . . . . . . . . . . . 154

5.10 Compare these two schemes in terms of $\mathrm{CO}_{2}$ emissions as the pollutant parameter of Retailer $1\left(\alpha_{1}\right)$ varies. . . . . . . . . . . 155

5.11 Heterogeneous networks powered by smart grids. . . . . . . . 156

5.12 Three stage Stackelberg game. . . . . . . . . . . . . . . . . . 160

5.13 Best response functions for homogeneous Bertrand duopoly. . . . . . 169

5.14 Utility function of one FBS versus its power allocation. . . . . . . 175

5.15 Power allocation of the FBS with respect to the interference price of-

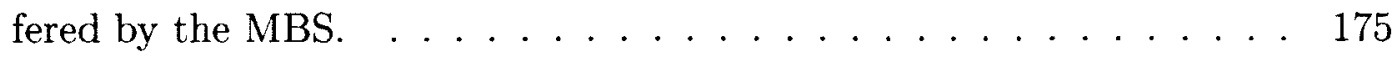


5.16 Utility function of the MBS with respect to the interference price offered by itself. . . . . . . . . . . . . . . . 176

5.17 Power allocation of the MBS with respect to its interference price. . . 177

5.18 Energy-efficient power allocation of the BSs with respect to the lowest prices offered by the retailers. . . . . . . . . . . . . . 178

5.19 Retailer 1's utility in a monoply situation. . . . . . . . . . 178

5.20 Convergence of the Stackelberg equilibrium. . . . . . . . . . . 179

5.21 Total electricity cost with respect to the lowest price offered by the retailers. . . . . . . . . . . . . . . . . . 180

5.22 Total $\mathrm{CO}_{2}$ emissions with respect to the lowest price offered by the retailers. . . . . . . . . . . . . . . . . . . 181 


\section{List of Acronyms}

\begin{tabular}{ll}
\hline Acronym & Definition \\
\hline \hline AMI & Advanced Metering Infrastructure \\
ANN & Artificial Neural Network \\
AP & Access Point \\
ARMA & Autoregressive Moving Average \\
AGWN & Additive Gaussian White Noise \\
BAN & Building Area Network \\
B-BID & Battery-Based Intrusion Detection \\
BPL & Broadband over Power Line \\
BS & Base Station \\
CIC & Customer Interruption Cost \\
CoMP & Coordinated Multipoint \\
CPP & Critical-Peak Pricing \\
CSR & Class Set Reduction \\
CSRR & Class Set Reordering \\
CSS & Cooperative Sensing Scheduling \\
DBSOS & Dynamic Base Station Operation Scheme \\
DE & Deployment Efficiency \\
DLC & Direct Load Control \\
&
\end{tabular}




\begin{tabular}{|c|c|}
\hline DP & Dynamic Programming \\
\hline $\mathrm{DR}$ & Demand Response \\
\hline DSL & Digital Subscriber Line \\
\hline DSM & Demand-Side Management \\
\hline $\mathrm{DT}$ & Decision Tree \\
\hline $\mathrm{EE}$ & Energy Efficiency \\
\hline FA & False Acceptance \\
\hline FAN & Field Area Network \\
\hline FAR & False Acceptance Rate \\
\hline FBS & Femtocell Base Station \\
\hline FFGS & Fossil-fuel Generation System \\
\hline $\mathrm{FN}$ & False Negative \\
\hline FNR & False Negative Rate \\
\hline FP & False Positive \\
\hline FR & False Rejection \\
\hline FRR & False Rejection Rate \\
\hline $\mathrm{HAN}$ & Home Area Network \\
\hline HIDS & Host-Based Intrusion Detection System \\
\hline HMM & Hidden Markov Model \\
\hline HRIDS & Host-Based Real Time Intrusion Detection System \\
\hline IAN & Industrial Area Network \\
\hline IBR & Inclining Block Rate \\
\hline IDS & Intrusion Detection System \\
\hline KKT & Karush-Kuhn-Tucer \\
\hline
\end{tabular}


LR Lagrangian Relaxation

MAB Multi-Armed Bandit

MANET Mobile Ad-Hoc Network

MBS Macro Base Station

MLR Monotone Likelihood Ratio

MMPP Markov-Modulated Poisson Process

MRROC Maximum Realizable ROC

MU Macro User

NAN Neighborhood Area Network

NWP Numerical Weather Prediction

OFDMA Orthogonal Frequency-Division Multiple Access

OpenADR Open Automated Demand Response

PAR Peak-to-Average Ratio

PDF Probability Distribution Function

PEC Pollutant Emission Cost

PLC Power Line Communication

POMDP Partially Observable Markov Decision Process

PVS Photo-Voltaic System

QoS Quality of Service

ROC Receiver Operating Characteristics

RTP Real-Time Pricing

RWP Random Waypoint

SCL Single Class Labels

SE Spectrum Efficiency 


\begin{tabular}{ll} 
SEP & Smart Energy Profile \\
SPE & Subgame Perfect Equilibrium \\
TOUP & Time-of-Use Pricing \\
UC & Unit Commitment \\
WTG & Wind Turbine Generator \\
XML & eXtensible Markup Language \\
\hline
\end{tabular}




\section{Chapter 1}

\section{Introduction}

Increasingly rigid environmental standards and rapidly rising energy prices have led to an increased research interest in reducing $\mathrm{CO}_{2}$ emissions and energy expenditure costs. Information and communications technologies (ICT) have a very significant role to play in addressing climate change globally and facilitating low carbon development. Since the carbon footprint is dominated by electricity consumption, an obvious way to reduce emissions is to increase energy efficiency and decrease energy consumption. In addition, the role of ICT includes not only the emission reduction and energy savings in ICT products and services, but also enabling low carbon emissions in other industries, such as electric power smart grids. This thesis studies stochastic schemes in green wireless cellular networks, green mobile ad-hoc networks (MANETs) and smart grids to make them more efficient, effective, and reliable.

In recent years, the demand for cellular data traffic has grown significantly with the introduction of mobile devices such as iPhone and iPad. Approximately 120,000 new base stations are deployed every year to service 400 million new mobile subscribers around the world [1]. Energy costs of a mobile service provider are almost half of its annual operating expenses [2]. The $\mathrm{CO}_{2}$ emissions produced by wireless cellular networks are equivalent to those from more than 8 million cars [3]. Hence, increasing the energy efficiency of future wireless cellular networks will reduce environmental 
impact and increase operators' profits [3].

Unlike infrastructured wireless networks (e.g., cellular networks), mobile adhoc networks (MANETs) do not rely on a fixed infrastructure for their operation. MANETs offer numerous advantages, such as use in situations where a fixed infrastructure is not available or too expensive. Because of their self-organising capabilities, MANETs can be rapidly deployed with minimum user intervention. Therefore, MANETs can be widely used in various applications. Supporting securitysensitive applications in hostile environments has become an important research area for MANETs. In MANETs, the nodes are often energy-constrained devices, since they are powered by batteries. Therefore, it is also important to improve the energy efficiency of MANETs.

On the other hand, the power grid infrastructure is experiencing a significant shift from the traditional electricity grid to the smart grid. The electricity demand of consumers has sharply increased in recent years. Moreover, there is increasing interest in integrating renewable resources into the power grid, in order to decrease carbon emissions. In addition, demand-side management (DSM), such as dynamic pricing, and demand response (DR) programs should be used to improve the reliability of the grid by dynamically changing or shifting the electricity consumption. These new requirements and aging existing grids make the modernization of the grid infrastructure a necessity.

\subsection{Problem Statement}

In high security green MANETs, network lifetime is a major concern, since most of devices are powered by batteries with limited capacity. In this thesis, network lifetime is defined as the time until all of the chosen nodes in the network are in the low-energy 
state. Security is another major concern for providing trusted communications in a potentially hostile environment. Existing methods try to improve the security performance of networks either using continuous user authentication or intrusion detection. However, these two kinds of methods have their limitations. Therefore, in a tactical environment, continuous user authentication and intrusion detection can be jointly used, in order to maximize the security performance of the MANETs. However, intrusion detection and continuous user authentication may consume a large amount of energy, which is a serious concern for energy-constrained devices in MANETs. Therefore, it is very important to schedule the intrusion detection and user authentication activities at each time slot, taking system security and energy into account. The proposed scheme should have low computational complexity to further reduce the energy consumption of the networks.

Smart grids can optimize electricity generation, transmission, and distribution, reduce peaks in power usage, and sense and prevent power blackouts, by incorporating network and information technologies with intelligent algorithms [4]. Therefore, smart grids have the potential to significantly improve the efficiency and reliability of the power grid. In order to minimize greenhouse gas emissions, it is optimal to always use electricity generated by the renewable energy generation systems. However, most renewable energy sources are highly intermittent in nature and uncontrollable. It is a challenging task to guarantee that the power demand load and power generation remain balanced, which is very important for system reliability. A mismatch between the supply and demand could cause a deviation of zonal frequency from nominal value, and power outages and blackouts may occur in the severe case $[5,6]$.

In smart grids, unit commitment (UC) and demand-side management (DSM) can be used to solve the above balance problem by adjusting the output electricity through scheduling power generation systems, and by controlling the electricity consumptions 
of customers. It is very important to consider the stochastic characteristics of renewable energy sources and the power demand loads in the UC problem in smart grids, which has been ignored in the current research. Appropriate prediction models for renewable energy and power demand used in the UC problem have the potential to improve the performance of the grid. Electricity retailers' procurement and price decisions also affect the reliability of smart grids. Therefore, retailers need to make effective decisions about electricity sources, the electricity amount they procure and the price they offer to their customers. These decisions need to be considered together, since one decision can affect another. However, these decisions are considered independently in the current research. Overall, it is very important to take reliability, the pollutant emissions and operational expenditure into consideration when proposing solutions for the above decision problems in smart grids.

In wireless cellular networks, one of the most important trade-offs is the trade-off between the energy efficiency and quality of service (QoS) for customers. For example, in order to minimize the service blocking probability, the base stations might have to be switched on all the time, which can consume a large amount of electricity. Therefore, it is very important for base stations to decide how many of stations need to be active in each cooperation cluster. In order to improve the energy efficiency of wireless cellular networks, new technologies such as heterogeneous networks and cognitive radios can be also used.

All of the problems mentioned above can be considered as decision problems. Each decision problem can be formulated as one or more optimization problems. It is very difficult to formulate and solve decision problems when the decisions have to be made under environmental uncertainties. Stochastic optimization tries to find solutions for these kinds of decision problems. It is very important to find a reasonable trade-off between the realism of the optimization model and the tractability of the problem [7]. 
In the field of stochastic optimization, the multi-armed bandit (MAB) models are very important, since they can be solved efficiently. MAB models can be used to optimally allocate one or more resources among a number of competing projects in a sequential manner [8]. These models are a powerful framework for solving distributed optimization problems.

In this thesis, we consider partially observable Markov decision process (POMDP) MAB problems (a.k.a. hidden Markov model (HMM) MAB problems), for the following reasons. Due to false acceptance (FA) and false rejection (FR) errors made in biometric authentication process, and false positive (FP) and false negative (FN) errors made in intrusion detection process, the security state of the system may not be observed perfectly. Therefore, we formulate the system as a partially observable system. In smart grids, hidden Markov models are used for modeling the intermittent renewable energy resources. Markov models are an effective approach to characterize the correlation structure of the renewable energy outputs [9-11]. Meteorological instability and complex system dynamics (e.g., wind turbine or solar cells malfunction) make it difficult to fully observe the renewable energy system state, so hidden Markov models are appropriate.

When a wireless cellular network is powered by smart grids, only considering energy efficiency in the cellular network may not be enough. Actually, in smart grids environment, consuming more energy can be better than consuming less energy in some circumstances. This is because large amounts of renewable energy will be integrated in the future smart grids. In addition, shut-down and ramp-up of a power plant can be costly or sometimes not technically viable, and the electricity storage capacities are limited in practice [12-14]. Indeed, power generators may use negative prices (i.e., consumers will be paid for consuming electricity) to encourage consumers 
to consume more energy in some situations $[12,15]$. Therefore, smart grids have a significant impact on the energy efficiency strategies of green wireless cellular networks, which has not been considered in the current research work.

In this thesis, game theory is used to model the significant impact of smart grids on the energy efficiency strategies of green wireless cellular networks. Game theory is a very important mathematical tool for analyzing the strategic interactions (such as conflict and cooperation) among multiple rational decision makers [16]. In recent years, the applications of game theory in wireless networks have been examined closely. One important game theory model used in this thesis is the Stackelberg game. In this kind of game, there are two different kinds of roles: leader and follower. The problem is to find an optimal strategy for each leader in order to maximize each leader's utility (i.e., the level of satisfaction), assuming that the followers maximize their utility given the leaders' actions. It is an appropriate model for the scenarios considered in this thesis: the electricity prices offered by retailers change frequently to reflect variations in the cost of the energy supply. The electricity retailers in smart grids decide the electricity prices offered to the customers first, and the customers then decide the amount of electricity to consume based on the electricity prices.

\subsection{Contributions and Publications}

Based on the work presented in Chapter 3, Chapter 4 and Chapter 5, several articles have been published, accepted or submitted. The contributions of this thesis are as follows:

- A comprehensive literature review on high security green MANETs, smart grids and green wireless cellular networks is provided in Chapter 2.

- We present a distributed scheme combining continuous user authentication and 
intrusion detection in Chapter 3. We use Dempster-Shafer theory for security and trust data fusion in high security green MANETs, which is a novel application of this theory. The proposed scheme can improve network security and also lower computational complexity. The contents of this chapter are published in the following journal papers and conference papers:

- S. Bu, F. R. Yu, P. X. Liu, and H. Tang, "Structural results for combined continuous user authentication and intrusion detection in high security mobile ad-hoc networks," IEEE Trans. on Wireless Comm., vol. 10, pp. 3064-3073, Sept. 2011.

- S. Bu, F. R. Yu, P. X. Liu, , P. Mason, and H. Tang, "Distributed combined authentication and intrusion detection with data fusion in high security mobile ad-hoc networks," IEEE Trans. on Veh. Tech., vol. 60, pp. 1025-1036, Mar. 2011.

- S. Bu, F. R. Yu, P. X. Liu, and H. Tang, "A computationally efficient method for joint authentication and intrusion detection in mobile ad-hoc networks," in Proc. IEEE ICC, (Kyoto, Japan), Jun. 2011.

- S. Bu, F. R. Yu, P. X. Liu, H. Tang, and P. Mason, "Distributed combined authentication and intrusion detection with data fusion in high security mobile ad-hoc networks," in Proc. IEEE Milcom, (San Jose, USA), Oct./ Nov. 2010.

- A distributed stochastic UC scheduling scheme and a novel game-theoretical decision-making scheme for electricity retailers are proposed in Chapter 4. The proposed UC scheduling scheme can significantly reduce the cost and greenhouse gas emissions. Using our proposed decision-making scheme, electricity retailers and customers can achieve a higher profit. The contents of this chapter are 
presented in the following journal papers and conference papers:

- S. Bu, F. R. Yu, and P. X. Liu, "A game-theoretical decision-making scheme in the smart grid with demand-side management: towards a smart cyber-physical power infrastructure," submitted to IEEE Transactions on Parallel and Distributed System (TPDS) SI on Cyber-Physical Systems (under revision), Sept. 2011.

- S. Bu, F. R. Yu, and P. X. Liu, "Distributed unit commitment scheduling in the future smart grid with intermittent renewable energy resources and stochastic power demands," submitted to International Journal of Green Energy (under revision), Oct. 2011.

- S. Bu, F. R. Yu, and P. X. Liu, "A game-theoretical decision-making scheme for electricity retailers in the smart grid with demand-side management," in Proc. IEEE SmartGridComm, (Brussels, Belgium), Oct. 2011.

- S. Bu, F. R. Yu, and P. X. Liu, "Dynamic pricing for demand-side management in the smart grid," in Proc. IEEE Online Conference on Green Communications, Sept. 2011.

- S. Bu, F. R. Yu, P. X. Liu, and P. Zhang, "Distributed stochastic scheduling in smart grid communications with intermittent renewable energy resources," in Proc. IEEE ICC Workshop on Smart Grid Communication, (Kyoto, Japan), Jun. 2011.

- S. Bu, F. R. Yu, and P. X. Liu, "Stochastic unit commitment in smart grid communications," in Proc. IEEE INFOCOM Workshop on Green Communications and Networking, (Shanghai, China), Apr. 2011. 
- A dynamic operation scheme for cellular base stations depending on the traffic, real-time electricity price and the pollutant level associated with electricity generation is proposed in Chapter 5. Heterogeneous networks, cognitive radios, and smart grids have also been combined to improve cellular network energy efficiency in that chapter. Research results show that smart grids have a significant impact on green wireless cellular networks. Our proposed schemes can signficantly reduce operational expenditure and $\mathrm{CO}_{2}$ emissions in green wireless cellular networks. The contents of this chapter are presented in the following journal papers, conference paper and book chapter:

- S. Bu, F. R. Yu, Y. Cai, and P. X. Liu, "When the smart grid meets energy-efficient communications: Green wireless cellular networks powered by the smart grid," IEEE Transactions on Wireless Communications (accepted), Mar. 2012.

- S. Bu, F. R. Yu, and P. X. Liu, "Energy-efficient cognitive heterogeneous wireless networks in the smart grid environment," submitted to IEEE Transactions on Vehicular Technology, Mar. 2012.

- S. Bu, F. R. Yu, Y. Cai, and P. X. Liu, "Energy-efficient cellular networks with CoMP communications and smart grid," to be presented in IEEE ICC-GCN, (Ottawa, Canada), Jun. 2012.

- S. Bu, F. R. Yu and P. X. Liu, "Green wireless cellular networks in the smart grid environment," in Green Communications and Networking (F. R. Yu, X. Zhang, and V. C. M. Leung, eds.), CRC Press, 2012. 


\subsection{Thesis Organization}

The rest of this thesis is organized as follows. In Chapter 2, a comprehensive literature review of high security green MANETs, smart grids and green wireless cellular networks is provided. Research findings in these three areas are described in Chapters 3, 4 and 5, respectively. Finally, Chapter 6 draws the conclusions and discusses future work. 


\section{Chapter 2}

\section{Overview of Green Wireless Networks and Smart Grids}

In high security green MANETs, user authentication is critical in preventing nonauthorized users from accessing or modifying network resources. Intrusion detection is also important in high security green MANETs to effectively identify malicious activities and so that the MANETs may respond appropriately. In a tactical environment, continuous user authentication and intrusion detection might need to be considered jointly to enable the performance of the MANETs to reach an acceptable level. Network lifetime also needs to be considered, since most of the network devices are powered by batteries with limited capacity.

In smart grids, one major challenge is how to increase the reliability of the grid. Most renewable energy sources are highly intermittent in nature and often uncontrollable, which induce significant fluctuations on the supply side of the power grid. The use of smart meters and smart appliances in smart grids can cause uncertainties on the demand side [17]. Therefore it is a challenging task to guarantee that the power demand load and power generation remain balanced, which is very important for system reliability. Two important approaches, unit commitment (UC) and demand-side management (DSM), try to solve the problem by scheduling power generation systems and controlling the electricity consumption of the user side, respectively. 
Another important type of wireless network considered in this thesis are wireless cellular networks. Three important methods (renewable energy resources, energyaware cooperative base station (BS) power management, and cognitive heterogeneous networks) are effective at making cellular networks energy efficient, as they reduce the electricity consumption of the cellular network while maintaining an acceptable level of network performance.

In the rest of this chapter, we will give an overview of these two kinds of green wireless networks, and of smart grids.

\subsection{High Security Green MANETs}

In this section, two important classes of approaches to MANET security (continuous user authentication and intrusion detection) and their related work are presented.

\subsubsection{Biometric-Based Continuous User Authentication}

Most authentication systems do not need to re-authenticate the users for continuous access to the protected resources. However, in hostile environments where the chances of a node falling into hostile hands are high, user authentication is needed not only for the initial login, but also to verify the presence of the authentic user continuously, in order to reduce the vulnerability of the system [18]. The frequency depends on the situation severity and the resource constraints of the network [18].

User authentication can be performed by using one or more types of validation factors: knowledge factors, possession factors and biometric factors. Knowledge factors, such as passwords, and possession factors, such as tokens, are very easy to implement but can make it difficult to distinguish an authentic user from an impostor if there is no direct connection between a user and a password or a token. Biometrics 
technology, such as the recognition of fingerprints, irises, faces, retinas, etc. provides possible solutions to the continuous user authentication problem in MANETs [19], since it has direct connection with user identity. Using this technology, individuals can be automatically and continuously identified or verified by their physiological or behavioral characteristics without user interruption $[19,20]$.

Biometric systems include two kinds of operation models: identification and authentication. In the proposed system, the biometric systems operate in authentication mode (one-to-one match process) to address a common security concern: positive verification (the user is whoever the user claims to be). Based on a comparison of the matching score between the input sample and the enrolled template with a decision threshold, each biometric system outputs a binary decision: accept or reject. In most real-world implementations of biometric systems, biometric templates are usually stored in a location remote to the biometric sensors [21].

In biometric authentication processes, two kinds of errors can be made: false acceptance (FA) and false rejection (FR). FAs result in security breaches, since unauthorized persons are admitted to access the system/network. FRs result in convenience problems, since genuinely enrolled identities are denied access to the system/network and maybe some further checks need to be done. The frequency of false acceptance errors and of false rejection errors are called false acceptance rate (FAR) and false rejection rate (FRR), respectively. The FAR can be used to measure the security characteristics of the biometric systems, since a low FAR implies a low possibility that an intruder is allowed to access the system/network. In tactical MANETs, failure in user authentication might result in serious consequences.

Some research has been done in continuous biometric-based user authentication. Muncaster et al. [22] used dynamic Bayesian networks for authentication. Sim et al. [18] presented the theory, architecture, implementation, and performance of a 
multimodal biometrics verification system, and also proposed new metrics against which they benchmark their system. Azzini et al. [23] presented a biometric method for continuous user authentication using a fuzzy controller. Altinok et al. [24] proposed a multimodal system that performs continuous authentication by integrating information temporally and across modalities. Klosterman et al. [25] presented a novel biometric-enhanced authentication system, which increases desktop security by using a trusted camera, message authentication codes, and continuous authentication.

\subsubsection{Intrusion Detection Systems}

Intrusion detection is a process of monitoring computer networks and systems for violations of security. Intrusion detection can be automatically performed by intrusion detection systems (IDSs). Two main technologies of identifying intrusion detection in IDSs are: misuse detection and anomaly detection [26]. Misuse detection is the most common signature-based technique, where incoming/outgoing traffic is compared against the possible attack signatures/patterns stored in a database. If the system matches the data with an attack pattern, the IDS regards it as an attack and then raises an alarm. The main drawback of misuse detection is that it cannot detect new forms of attacks. Anomaly detection is a behavior-based method, which uses statistical analysis to find changes from baseline behavior. This technology is weaker than misuse detection, but has the benefit of catching the attacks without signature existence [26].

Multiple algorithms have been applied to model attack signatures or normal behavior patterns of systems. Three common algorithms are naive Bayes, artificial neural network (ANN), and decision tree (DT) [27]. A naive Bayes classifier is based on a probabilistic model to assign the most likely class to a given instance. ANN is a pattern recognition technique, with the capacity to adaptively model user or 
system behavior. DT, a useful machine learning technique, is used to organize the attack signatures into a tree structure. Most of the IDSs only use one of the above algorithms.

IDSs can be categorized as follows [28]: (i) Network-based intrusion detection, which runs at the gateway of a network and examines all incoming packets. (ii) Router-based intrusion detection, which is installed on the routers to prevent intruders from entering the network. (iii) Host-based intrusion detection, which receives the necessary audit data from the host's operating system and analyzes the generated events to keep the local node secure. For MANETs, host-based IDSs are suitable since no centralized gateway or router exists in the network [26].

IDSs can make two kinds of errors: false positive (FP) and false negative (FN). FNs result in security breaches, since intrusions are not detected, and therefore no alert is raised. The false negative rate (FNR) can be used to measure the secure characteristics of the IDSs, since a low FNR implies a low possibility that intrusion occurs without detection.

Some research has been done in host-based IDSs. Hu et al. [29] proposed a data pre-processing method to improve a hidden Markov model (HMM) training for hostbased anomaly intrusion detection. Jacoby et al. [30] presented a mobile battery-based intrusion detection (B-BID) method to correlate attack activities with device power consumption patterns. Chari et al. [31] presented their experiences with building BlueBox, a policy-driven host-based intrusion detection system. Leu et al. [32] proposed a host-based real time intrusion detection system (HRIDS), where cooperative attacks submitted by users simultaneously can be discovered by using data mining and forensic techniques. Liu et al. [33] proposed a game-theoretical model to reduce the resource consumption of a host-based intrusion detection system (HIDS) without sacrificing the detection accuracy, by dynamically adjusting the objects a HIDS 
monitors and the monitoring time. Khreich et al. [34] presented a multiple-HMM approach to improve anomaly detection, where each HMM is trained using a different number of hidden states capturing different temporal structures of the data, and the responses are then combined in the receiver operating characteristics (ROC) space according to the maximum realizable ROC (MRROC) technique.

\subsubsection{Combined Continuous User Authentication and Intru- sion Detection}

Continuous user authentication and intrusion detection can be considered jointly to further improve the performance of high security green MANETs. Some research has been done in combining intrusion detection and continuous authentication in MANETs [35]. The framework proposed in [35] is shown to be effective as it combines an important prevention-based security approach and a detection-based approach. However, the scheme proposed in [35] is a centralized scheme, in which the whole network is formulated as a single partially observable Markov decision process (POMDP). Therefore, the scheme is more suitable for a single node rather than a network with distributed nodes. Since the state space of the POMDP in [35] grows exponentially with the number of biometric sensors and IDSs, solving the POMDP can be computationally intractable [8]. Therefore, it is difficult to implement the scheme proposed in [35] for a MANET with distributed nodes.

\subsection{Smart Grids}

In this section, two efficient methods to improve the reliability of smart grids, UC and DSM, are described. The related work is also presented. The major smart grid standards supporting DSM are also described. Following this, we give an overview of 
the decision-making of electricity retailers in the electricity market, which also affects the reliability of smart grids. It is crucial to have two-way communication systems in smart grids. Therefore, in this section, we introduce network architecture for smart grids, and major requirements for smart grid communication infrastructures.

\subsubsection{Unit Commitment}

UC scheduling of power generation systems is an important issue in smart grids to effectively coordinate energy demand and generation in order to minimize cost and greenhouse gas emissions, and to avoid blackouts in the event that the natural phenomena necessary to generate renewable energy do not occur [36].

Many efforts have been made to develop efficient and robust UC algorithms for the traditional power grid by researchers. Ongsakul et al. [37] proposed enhanced adaptive Lagrangian relaxation for UC. Pappala et al. [38] proposed a variable-dimension optimization approach to address the high dimensionality issues in solving the UC problem and implemented the proposed approach in particle swarm optimization algorithm. Simopoulos et al. [39] proposed an enhanced simulated annealing algorithm for solving the UC problem. Chung et al. [40] developed an advanced quantuminspired evolutionary UC algorithm by developing a new priority-list-based initialization method and improved search strategies to improve the algorithm for large-scale $\mathrm{UC}$ problems. In their work, they did not take renewable energy into account during the planning process. The fluctuation of renewable energy sources makes it difficult to optimize the usage of renewable energy. Therefore, a large level of penetration of renewable energy will present many challenges to the unit commitment operation in smart grids.

There is some work related to UC scheduling in renewable energy generation. 
Wang et al. [41] proposed a security-constrained stochastic UC formulation that accounts for wind-power volatility together with an efficient Benders decomposition solution technique. Tuohy et al. [42] reported a detailed closed-loop stochastic UC formulation. Pappala et al. [43] used ANN models to compute forecasts and confidence intervals for the total aggregated power for a set of distributed wind generators. Xie et al. [44] studied model predictive dispatch of wind generation, where the future wind generation is described by an autoregressive moving average (ARMA) model. Methaprayoon et al. [45] used ANN models to forecast wind power, and integrated the forecast results into UC scheduling. Constantinescu et al. [46] presented a computational framework for integrating a numerical weather prediction (NWP) model to perform stochastic unit commitment/economic dispatch studies with wind-power adoption. Bialek et al. [47] proposed risk-limiting dispatch, where the scheduling with known demand and uncertain supply is treated as a multi-stage decision process with recourse.

Although some work has been done about UC in smart grids, most previous work uses the current observed power output from renewable energy resources to make scheduling decisions for subsequent energy generation. Moreover, most existing work assumes the independence of successive wind speeds or solar radiation levels when they use various probability distribution functions to predict the magnitude of the wind speed or solar radiation [48]. In addition, most previous work does not predict the electricity demand or even assumes that it is known in the UC. These assumptions may not be realistic in practice. Therefore, appropriate prediction models of renewable energy and power demand are needed in the UC in the future smart grid with the large adoption of renewable energy.

In order to effectively model renewable energy, the successive-time state transition probabilities need to be known $[9,10]$. Weibull, Rayleigh, lognormal or Gamma 
probability distribution functions (PDFs) are often used to predict the magnitude of the wind speed or solar radiation [49-53]. However, these theoretical PDFs assume independence of successive wind speeds or solar radiation levels [48], which may not be realistic in practice. Finite-state Markov models have been widely accepted in the literature as an effective approach to characterize the correlation structure of the renewable energy outputs [9-11]. Considering Markov models may enable substantial performance improvement over the schemes with memoryless models. Moreover, it is generally assumed that the renewable energy system state is known perfectly in previous work. However, meteorological instability and complex system dynamics (e.g., wind turbine or solar cells malfunction) make it difficult to fully observe the system state. Therefore, HMMs are more efficient in modeling renewable energy systems $[54,55]$.

\subsubsection{Demand-Side Management and Supporting Standards in Smart Grids}

\subsubsection{Demand-Side Management}

DSM, an important mechanism for improving the reliability of smart grids, is a set of programs implemented by utility companies that allow customers a greater role in dynamically changing or shifting the electricity consumption [56]. DSM can help utilities operate more efficiently, reduce emission of greenhouse gases, and also decrease the cost for electricity consumers. DSM programs include demand response (DR) programs, energy efficiency programs, and conservation programs. DR programs transfer customer load during periods of high demand to off-peak periods and can reduce critical peak load or daily peak demand. DR programs can be divided into three kinds of major programs: time-based DR programs (e.g., smart pricing), incentive-based DR 


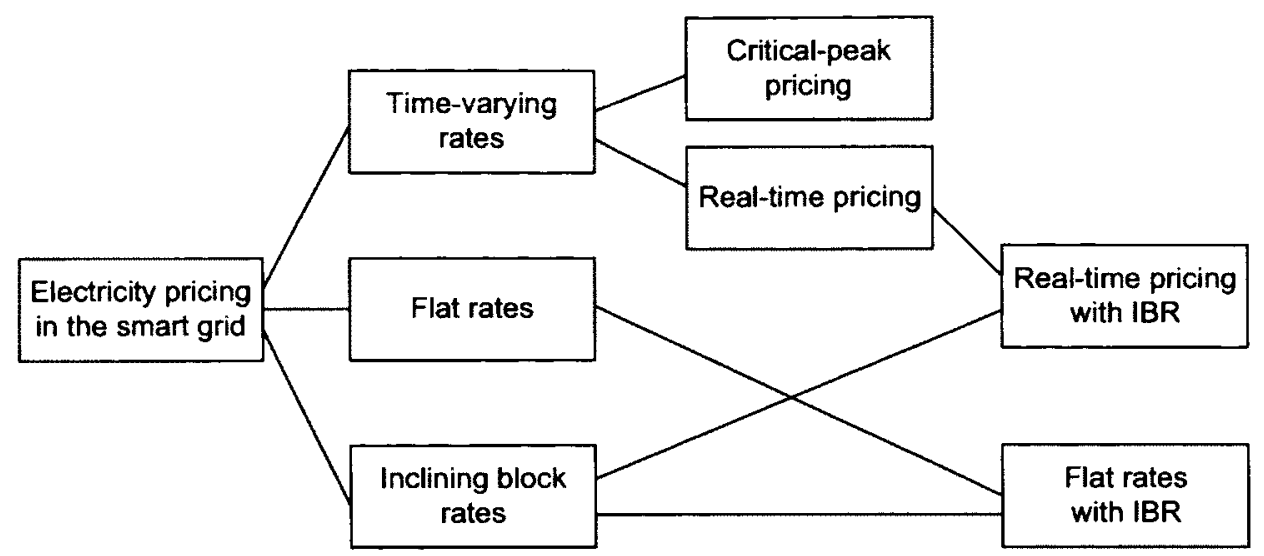

Figure 2.1: Smart electricity pricing models in smart grids.

programs, and load shifting and control programs. Different kinds of programs can be implemented together in real systems to further improve demand management performance. Recently, smart pricing programs (e.g., real-time pricing) have attracted much attention as one of the most important DSM strategies to encourage users to consume electricity more wisely and efficiently [57].

Several smart pricing models have been proposed in smart grids to reduce the users' electricity costs and the peak-to-average ratio (PAR) in the load demand. Inclining block rates (IBRs), critical-peak pricing (CPP) and real-time pricing (RTP) are three important ones. In the IBR model, the electricity price increases to a higher value when the total residential load is beyond a certain threshold [58]. This pricing model has been adopted by some utility companies, such as British Columbia Hydro Company in Canada. In CPP, the price is decided in advance based on the demand hours. For example, peak price, mid-peak price and off-peak price are used by Ontario Hydro Company in Ottawa, Canada [59]. In RTP, the exact price value is calculated in real-time, and announced only at the beginning of each time period. Different smart electricity pricing models in smart grids are illustrated in Fig. 2.1.

Among the various classes of DSM programs, RTP models have the potential to lead to economic and environmental advantages. The pricing schemes used to 


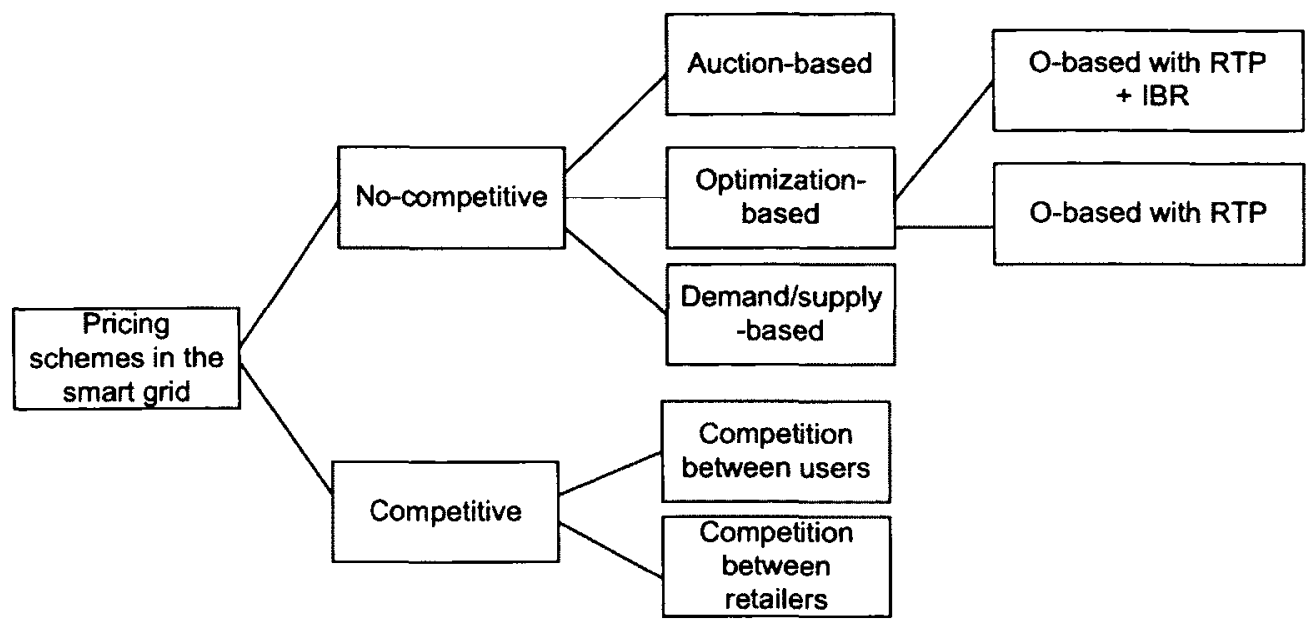

Figure 2.2: Pricing schemes to develop RTP models in smart grids.

develop RTP models in smart grids can be divided into two categories based on the economic environment. In the non-competitive environment, schemes include auction-based, optimization-based, and demand/supply-based. In the competitive environment, schemes are divided based on whether the competition is between users or between retailers. The schemes for RTP models in smart grids are illustrated in Fig. 2.2.

There is some work in the literature about RTP in smart grids. Holland et al. [60] discussed how real-time pricing can potentially reduce the emission levels of $\mathrm{SO}_{2}$, $\mathrm{NO}_{x}$, and $\mathrm{CO}_{2}$ in the United States. Mohsenian-Rad et al. [58] proposed a computationally feasible and automated optimization-based residential load control scheme in a retail electricity market, combining RTP with IBRs, and they also considered the real-time price prediction on the user side. Samadi et al. [57] proposed a RTP algorithm, which can maximize the aggregate utility of all users in the system and minimize the cost imposed to the energy provider while keeping the total power consumption below the generating capacity. Mohsenian-Rad et al. [61] proposed a smart pricing tariff such that the interactions among the users automatically lead to an optimal aggregate load profile at the equilibrium of an energy consumption scheduling 
game.

\subsubsection{Smart Grid Standards Supporting Demand-Side Management}

In this subsection, we will describe the important smart grid standards that are relevant to DSM [62].

Open automated demand response (OpenADR) provides an open standardized DR interface and communication data models that allow utilities to communicate standard DR signals, including price, reliability and event signals, to trigger customers' pre-programmed energy management strategies. DR signals can be sent directly to electric customers using eXtensible Markup Language (XML) and existing communication systems such as the Internet. In its early phases, OpenADR has been developed by Lawrence Berkeley National Laboratory. The specification was released in Apr. 2009. An OpenADR Alliance was formed in Oct. 2010 to accelerate the development, adoption and compliance of OpenADR standards throughout the energy industry and to provide a common language for smart meters. It has low cost and can improve the reliability, robustness and cost-effectiveness of DR in commercial buildings. With OpenADR, utilities can offer electricity pricing programs based on automated DR, which can reduce peak power use [63].

ZigBee/Home Plug Smart Energy Profile 2.0 (SEP 2.0) can be used to transmit data between smart meters and devices in the home over a variety of networks, including ZigBee, Home Plug power line, and Wi-Fi. It enables wireless communication between utility companies and household devices, and improves energy efficiency by allowing consumers to manage their energy consumption with near real-time information. SEP 2.0 is a significant update of the existing Smart Energy Profile 1.0, which only supports ZigBee. However, these two versions are not compatible.

$B A C n e t$ is designed for building automation and control networks. It provides a 
mechanism for building automation devices to exchange information, no matter which kind of building service they perform. BACnet defines a number of data link layers, such as token-passing and point-to-point data link layer, a number of services used for communication between building devices, and a number of objects acted upon by the services. BACnet can be used for very small systems to multi-building operations. It is also a standard protocol supported by different vendors and user communities.

ANSI C12 Metering includes ANSI C12.18, C12.19, C12.21 and C12.22. C12.19 specifies the data structure (tables) for metering. C12.18, C12.21 and C12.22 specify how to transmit meter data over a local optical port, telephone modems and a generic wide-area network, respectively. C12.18 and C12.21 are widely used in commercial and industrial meters, but they are too expensive for meter reading of residential meters. C12.22 provides a mechanism for exchanging the meter data between two nodes without considering the underlying transport. These C12 standards are mature and well-accepted by most of the metering vendors in multiple industries. However, it is complicated to achieve interoperability on the customer side.

LONWorks (ANSI/EIA/CEA 709) is a general purpose local area networking protocol. This standard includes four parts: EIA 709.1-B (the control network protocol), IEC 709.2 (power line carrier physical layer), EIA 709.3 (twisted pair physical layer) and EIA 709.4 (fiber optic physical layer). The protocol can be operated at different rates based on the used media. It is widely used for various applications, including electric meters, home automation and building automation. However, it is only a "de facto" standard and its object model is not structured.

\subsubsection{The Decision-Making of Electricity Retailers}

In an electricity market, retailers procure electricity from various electricity sources (e.g., the pool, electricity derivatives, and self-production units), and then sell it 
to customers [64]. These electricity sources have different characteristics, and these characteristics might change over time. For example, the pool price is uncertain and volatile, and the costs of the other electricity source options are generally higher than the expected pool price $[64,65]$. In smart grids, renewable energy sources are also integrated into the power grid. Therefore, there is a trade-off between different electricity sources. On the other hand, the electricity demand of the customers might vary with time. Therefore, retailers need to make effective decisions about electricity sources, the electricity amount they procure and the price to offer to the customers.

There is some work in the literature related to retail electric power operations. Triki et al. [66] discussed the RTP with an adjustable customer base line. Carrion et al. [67] presented a stochastic programming methodology to determine the optimal retail price based on fixed pricing and the amounts of power procured from the pool and forward contracts. Karandikar et al. [68] used a capital asset pricing model to determine the electricity prices for retailers. Celebi et al. [69] developed a computable equilibrium model to estimate the time-of-use (TOU) rates based on the costs of different power generation. Yusta et al. [70] discussed how different price strategies affect the retailers' profit. Gabriel et al. [71] analyzed a set of strategies available to the retailers to determine the forward loads. Gabriel et al. [72] proposed a stochastic optimization model to determine the optimal forward loads and retail price.

Most of the existing work mainly focuses on one or two decisions, price decision, electricity source decisions or the electricity amount they procure decisions, which need to be made by retailers. These strategies are mainly for the retailers in the traditional power grid. To the best of our knowledge, no work has been done for making optimal decisions about electricity sources and the amount that retailers should procure and the price to offer to the customers in smart grids, especially when RTP DSM is used. It is also very important to analyze how the retailer interacts with 
its customers, and how its decisions affect the customers' satisfaction level.

\subsubsection{Smart Grid Communication Infrastructures}

With a communication infrastructure, smart meters and sensor networks can provide real-time energy consumption and network status. Smart grids can use these communication systems to control intelligent appliances on the electricity users' side. A network operation center can optimize electricity generation and distribution, based on customers' electricity consumption data and online market pricing [73]. Therefore, the communication systems enable saving energy, increasing system reliability and operation transparency, and reducing expense [73].

In smart grids, the two-way communication system usually consists of a high-speed backbone and low-speed access networks. For the backbone, fiber optics, microwave radios, and cellular networks are good candidates. For the access networks, power line communications, twisted-pair lines, and wireless systems may be used.

\subsubsection{Network Architecture for Smart Grids}

Smart grid communications can be divided based on the areas that the network serves: local area networks, neighborhood/field area networks, and wide area networks $[62,74,75]$.

\section{Local Area Networks}

Local area networks include home area networks (HANs), building area networks (BANs) and industrial area networks (IANs). Power consumption devices and the connected smart meter can establish such a network, and the power consumption can be measured and collected by the smart meter. The main standards for local 
area networks include ZigBee, Wi-Fi, Home Plug, and X10. ZigBee (IEEE 802.15.4) is designed for industrial and home automation or security applications. Its power requirement and cost are low, and its scalability is good. However, the data rates are low and the transmission range is limited. Wi-Fi (IEEE 802.11) became a standard for laptops and subsequently phones. However, when using it in utilities, Wi-Fi's power consumption is an issue that needs to be considered carefully. Home Plug is a broadband over power line (BPL) system. It has QoS features and connectivity to home wiring. However, Its standardization level is low. X10 is a popular power line carrier system for home automation. The implementation costs are low if devices already use power lines. However, it is difficult to be used to support other kinds of traffic, such as Internet protocols.

\section{Neighborhood Area Networks or Field Area Networks}

Each neighborhood area network (NAN) is established among smart meters in HANs using short-range communication technologies. Similarly, each field area network (FAN) is composed of BANs or IANs. The main communication protocols include WiMAX, wired Ethernet, and power line communication (PLC) including broadband over power line (BPL) and narrowband PLC. WiMAX does not require expensive wired infrastructures. However, WiMAX has yet to be deployed at scale, which means some risks when applied to utilities. Wired Ethernet (IEEE 802.3) can be used to connect customers' sites to WAN or other networks. The cost is relatively low. However, it is only a local area network technology. BPL makes use of existing power lines, which removes the need to build new infrastructure (especially expensive in rural areas). Narrowband PLC is an international standard. However, PLC is expensive to use, and depends on current on the power lines. 


\section{Wide Area Networks}

Fiber optics, microwave radios, and cellular networks are the main candidates for backhaul networks. Fiber optics provides high scalability and bandwidth, but is expensive. A major advantage of using public cellular networks for smart grids are the reduction of the costs (by not having to build a new network and by leveraging the expertise of the telecom world). However, since public cellular networks are not specialized in machine-to-machine area, some requirements in utilities may not be met by cellular networks.

\subsubsection{Major Requirements for Smart Grid Communication Infrastruc- tures}

In order to design a new communication infrastructure to support smart grids, a lot of effort has to be put in understanding its requirements. In the following, we present the major smart grid communication requirements.

\section{Security}

In smart grids, large amounts of data, which are relevant for billing purposes and grid control, are considered critical for power utilities [76]. Therefore, securely communicating this data is very important. For smart grids, cyber security needs to address deliberate attacks, and non-deliberate attacks, such as equipment failures, user errors and natural disasters, etc. Cyber security has become a critical and challenging issue in smart grids, especially as smart grids become more interconnected. Efficient security mechanisms need to be developed to avoid cyber attacks. 


\section{System Reliability, Robustness and Availability}

The communication infrastructures in smart grids need to be highly reliable, since the supporting power systems have high reliability requirements. Availability of communication infrastructures will have a significant impact on smart grids' performance. The deployment of modern information and communication technology will significantly improve the system reliability and robustness [77]. Therefore, a hybrid communication technology mixed with wired and wireless solutions will be used to strength system reliability, robustness and availability, also taking installation costs into consideration [78].

\section{Scalability}

In smart grids, a large number of devices, such as smart meters, smart sensors and smart data collectors etc., need to join the communication network. Even though the commands and data packets related to each device are usually short, the total data volume transmitted in the network can be significant [79]. Therefore, smart grids need to well deal with the scalability.

\section{Quality-of-Service (QoS)}

In smart grids, communication between electricity suppliers and electricity users is critical. In smart grids, online sensor/meter reading data and power system control signals need to be transmitted in a real-time manner [73]. Therefore, the communication infrastructures should have efficient bandwidth and low latency. Performance degradation, such as delay, jitter or outage might compromise the system stability. The following methods have been proposed to satisfy the QoS requirement in smart 
grids: a QoS routing protocol [78], forecasting load variations [80], multi-hop routing using PLC [81], and smart monitoring using sensors [82].

\section{Interoperability}

Without a framework of interoperable standards for communications, it would be very difficult to make the smart grid system a reality. Since the potential standards landscape is very large and complex, interoperable standards adoption is challenging. Many utilities and regulatory groups are collectively trying to address interoperability issues through workgroups such as the GridWise Architecture Council and Open Smart Grid (Subcommittee of the Utility Communications Architecture International Users Group) as well as through policy action from NIST. In June 2009, National Institute of Standards and Technology (NIST) announced an interoperability project via IEEE P2030, which seeks to define interoperability of energy technology and information technology operations with electric power systems and end-user applications and loads.

\subsection{Green Wireless Cellular Networks}

In this section, three important energy-efficient solutions and the related work are presented. Four different trade-offs are also described, since they have a significant impact on the energy-efficient solutions.

\subsubsection{Energy-Efficient Solutions}

Three important energy-efficient solutions for cellular networks are as follows: renewable energy resources, energy-aware cooperative BS power management and cognitive 
heterogeneous networks.

\subsubsection{Renewable Energy Resources}

Powering BSs with renewable energy resources, especially in off-grid sites, could reduce greenhouse gas emissions and expenditures for cellular companies. Renewable energy sources, such as wind and solar power, are environment-friendly, since they do not produce greenhouse gases in the course of energy generation. They can also reduce the amount of electricity taken from the power grid. In off-grid sites, where expensive diesel powered generators are generally used, renewable energy resources can be a viable option to reduce the overall network expenditure [83]. Moreover, using air cooling and cold climates to cool the electronic devices in the BSs can further reduce the electricity consumption [84]. Therefore, a number of solutions, such as the Nokia Siemens Networks Flexi Multiradio base station and the Huawei green base station, are offered by BS equipment manufactures to reduce electricity consumption and support off-grid BSs with renewable energy resources $[85,86]$. However, the renewable energy sources cannot be the sole electricity source for a BS, since BSs require high reliability, and any power shortage will disturb the network's service provision [87].

\subsubsection{Energy-Aware Cooperative BS Power Management}

The traffic load in a cellular network can have significant spatial and temporal fluctuations due to a number of factors such as user mobility and activities [88]. Therefore, some cells might be under low traffic load, while others may be under heavy traffic load. Since operating a BS consumes a considerable amount of electricity, selectively switching off some BSs or some of the resources of an active BS under low load conditions can save a substantial amount of energy. When some cells are switched off or in a sleep mode, the radio coverage and service provision for these cells can be guaranteed 
by the remaining active cells. Network-level power management is required where multiple BSs coordinate together. Cell zooming is an important technique through which BSs can adjust their cell sizes according to the network or traffic situation, and therefore reduce the energy consumption of the whole network [88].

There are a few studies on saving energy using energy-efficient approaches in the operation of base stations. Marsan et al. [89] and Chiaraviglio et al. [90] analyzed the dynamic energy-efficient management scheme as the traffic varies over time. Zhou et al. [91] proposed BS energy saving algorithms, which can dynamically minimize the number of the active BSs with respect to spatial-time traffic variation. Jardosh et al. [92] proposed the adoption of resource on-demand strategies to power on or off WLAN access points (APs) dynamically based on the volume and the location of users' demand. Cao et al. [93] analyzed the energy saving performance of coordinated multipoint (CoMP) transmission and wireless relaying with an average outage constraint.

\subsubsection{Cognitive Heterogeneous Networks}

The deployment of heterogeneous networks based on smaller cells is an important technique to increase the energy efficiency of wireless cellular networks [83]. In recent years, the demand for cellular data traffic has grown significantly with the introduction of mobile devices. Macrocells have been used to provide large area coverage and to better handle user mobility in cellular networks. However, they are not efficient in providing high data rates. Recently, femtocells have been deployed to provide higher data rates and also enhance in-building coverage. Due to their small coverage area, femtocells require much less transmission power than macrocells, and therefore their BSs are much more energy efficient in providing broadband coverage [83]. However, a large number of femtocell BSs may increase the handoff rates of mobile users 
among adjacent cells, and also degrade the energy efficiency of the whole network [87]. Therefore, a deployment of BSs with different cell sizes is desirable in energy-efficient networks.

Cognitive radio will play an important role in improving energy efficiency for future wireless cellular networks [94]. Cognitive radio technology was originally proposed to improve spectrum efficiency [95]. Cognitive radio is used to collect information on the spectrum usage and to try to access the unused frequency bands intelligently. However, spectrum efficiency does not mean energy efficiency, and Shannon's capacity formula shows the trade-off between the bandwidth and power [96]. Therefore, in order to reduce power, the spectrum should be managed optimally and dynamically [94]. Holland et al. [97] mentioned that up to $50 \%$ of power can be saved if the operator dynamically manages its spectrum. However, cognitive radios are still in their infancy when it comes to improve energy efficiency for cellular networks.

There is some research on energy efficiency in heterogeneous networks or cognitive radio networks. Ashraf et al. [98] proposed an energy saving procedure to completely switch off the femtocell base station's radio transmission and associated processing, when it is not involved in an active call. Hou et al. [99] analyzed the power consumption of a combined cellular and femtocell wireless network architecture and its QoS support levels. In order to achieve energy efficiency in cognitive radio network, Zhang et al. [100] used the framework of POMDP to study the cooperative sensing scheduling (CSS) problem and parameter design problem. Hasan et al. [101] presented a solution to an energy-efficient resource allocation problem for maximizing the cognitive radio link capacity, by taking into account the availability of sub-bands and total allowed inference limit to the adjacent primary user (PU) bands. Ko et al. [102] studied the issues of resource sharing and access control in two-tier orthogonal frequency-division multiple access (OFDMA) femtocell networks. Lasaulce et 
al. [103] analyzed the effect of hierarchy in energy-efficient power control games both on the individual user and overall network performance in wireless networks. Treust et al. [104] studied energy-efficient distributed power control in cognitive radio networks and used a repeated game to formulate the optimal power control problem. Buzzi et al. [105] proposed a noncooperative power control game for maximum energy efficiency with a fairness constraint on the maximum received powers secondary users (SUs).

\subsubsection{Key Trade-offs in the Wireless Cellular Networks}

The following four key trade-offs need to be considered when designing energy-efficient solutions for cellular networks [106].

The trade-off between deployment efficiency and energy efficiency can be used to balance the deployment cost, throughput, and energy consumption in the network as a whole [106]. Deployment efficiency, defined as system throughout per unit of deployment cost, is an important measure of network performance for mobile operators [106]. These two metrics often lead to opposite design criteria for network planning. For example, in order to save expenditures on base station equipment and maintenance, network planning engineers favor making individual cell coverage as wide as possible. However, radio resource management engineers prefer the deployment of small cells in order to minimize energy radiation.

The trade-off between spectrum efficiency and energy efficiency can be used to balance the achievable rate for a given bandwidth and energy consumption of the network [106]. Spectrum efficiency, a measure of the system throughput per unit of bandwidth, is normally used as optimization objective for wireless networks [106]. Spectrum efficiency and energy efficiency are sometimes in conflict. Therefore, it is important to investigate how to balance these two important metrics. 
The trade-off between bandwidth and power can be used to balance the bandwidth utilized and the power needed for transmission for a given data transmission rate [106]. In wireless communications, bandwidth and power are two most important resources, however, they are very limited. The fundamental bandwidth-power relation shows that in order to increase energy efficiency at a given data transmission rate, the transmit power must decrease, and therefore the signal bandwidth needs to undergo a corresponding increase.

The trade-off between delay and power can be used to balance the average endto-end service delay and average power consumed in transmission [106]. Delay, also known as service latency, is used to measure QoS and user experience in the network.

\subsection{Conclusion}

This chapter provides a comprehensive survey of high security green MANETs, smart grids and green wireless cellular networks.

In high security green MANETs, the proposed algorithms and schemes tried to improve the security performance of the MANETs either using biometric user authentication or intrusion detection systems. Since each class of security approach has its limitations, there is a need to consider these two methods jointly to further improve the security performance of high security green MANETs, also taking network lifetime into account.

In smart grids, the proposed schemes for the UC problem either use the current observed power output from renewable energy resources to make scheduling decisions for subsequent energy generation, or do not predict the electricity demand or even assume that it is known in the UC process. These assumptions may not be realistic in practice. Therefore, there is a need to propose a stochastic UC scheduling scheme for 
smart grids considering the intermittent characteristics of renewable energy resources and stochastic power demand loads. In the decision-making schemes for electricity retailers, the existing work mainly focuses on a subset of the decisions made by retailers, and the strategies are mainly for retailers in the traditional power grid. Therefore, there is a need to research the full retailer decision process, especially when RTP DSM is used.

In considering the energy efficiency of wireless cellular networks, there is a need to also consider smart grids, since smart grids have a significant impact. However, this important perspective has been ignored in the energy efficiency research on wireless cellular networks. 


\section{Chapter 3}

\section{Distributed Combined User}

\section{Authentication and Intrusion Detection in High Security Green Mobile Ad-Hoc Networks}

\subsection{Introduction}

One important issue considered in this chapter is how to combine continuous user authentication and intrusion detection systems (IDSs) in a distributed manner in high security green mobile ad-hoc networks (MANETs). Continuous user authentication is an important prevention-based approach to protect high security MANETs. IDSs are also important in MANETs to effectively identify malicious activities. Considering these two approaches jointly is effective in optimal security design taking into account system security requirements and resource constraints in MANETs.

The highlights of the research contribution in this chapter are as follows:

- User authentication or IDSs are scheduled in a distributed manner considering both the security situations and resources (e.g., node energy) in MANETs. Since a biometric authentication process require a large amount of computation, the energy consumption is significant. Moreover, due to the dynamic wireless 
channels in MANETs, the energy consumption for data transmissions is changing dynamically (e.g., because of power control). Therefore, in the proposed scheme, energy consumption is also considered in order to improve the network lifetime. The proposed scheme does not require centralized controllers, and it is more generic and flexible than a centralized scheme in MANETs.

- Multimodal biometrics are deployed to alleviate the shortcomings of unimodal biometric systems. Since each device in the network has measurement and estimation limitations, more than one device can be chosen, and their observations can be fused to increase observation accuracy. Dempster-Shafer theory [107] is used for data fusion.

- The distributed continuous user authentication and intrusion detection scheduling problem is formulated as a partially observable Markov decision process (POMDP) multi-armed bandit problem.

- We first present a value iteration-based solution for obtaining the scheduling policy. For a large network with a variety of nodes, the value iteration-based solution can become computationally intractable [8]. We then present a structural results method for solving the scheduling problem in a large network. We show that, under reasonable conditions on MANETs, structural results can be derived for the combined continuous user authentication and intrusion detection problem, which are trivial to implement and make the solution useful in practice.

- Simulation results are presented to show the effectiveness and the performance of the proposed scheme.

The work presented in this chapter has resulted in two journal papers $[108,109]$ 
and two conference papers [110,111]. Possible future work in this area is provided in Chapter 6.

\subsection{Distributed Combined User Authentication and Intrusion Detection with Data Fusion}

This section studies distributed, combined user authentication and intrusion detection with data fusion in high security green MANETs. This section is organized as follows. Section 3.2.1 describes the system model. Section 3.2.2 shows how to use DempsterShafer theory for the fusion of IDSs and biometric sensors. The integrated system is formulated in Section 3.2.3. Section 3.2.4 shows some simulation results.

\subsubsection{System Model}

Assume that a MANET has a continuous biometric-based authentication system with $N-W$ biosensors and $W$ IDSs, which have the ability to detect intrusions. The IDSs are also modeled as sensors bringing the total number of sensors to $N$. Without loss of generality, we assume that some nodes have one or more biosensors, and some do not have any biosensor due to the heterogeneity of network nodes in the MANET. Similarly, some nodes are equipped with the IDS, and some are not equipped with the IDS. The total number of network nodes in the MANET is not directly related to the number of sensors. An example framework for the MANET with biosensors and IDSs is illustrated in Fig. 3.1.

The system can perform two kinds of operations: intrusion detection and user authentication. The IDSs can continuously monitor the system. Authentication may be 


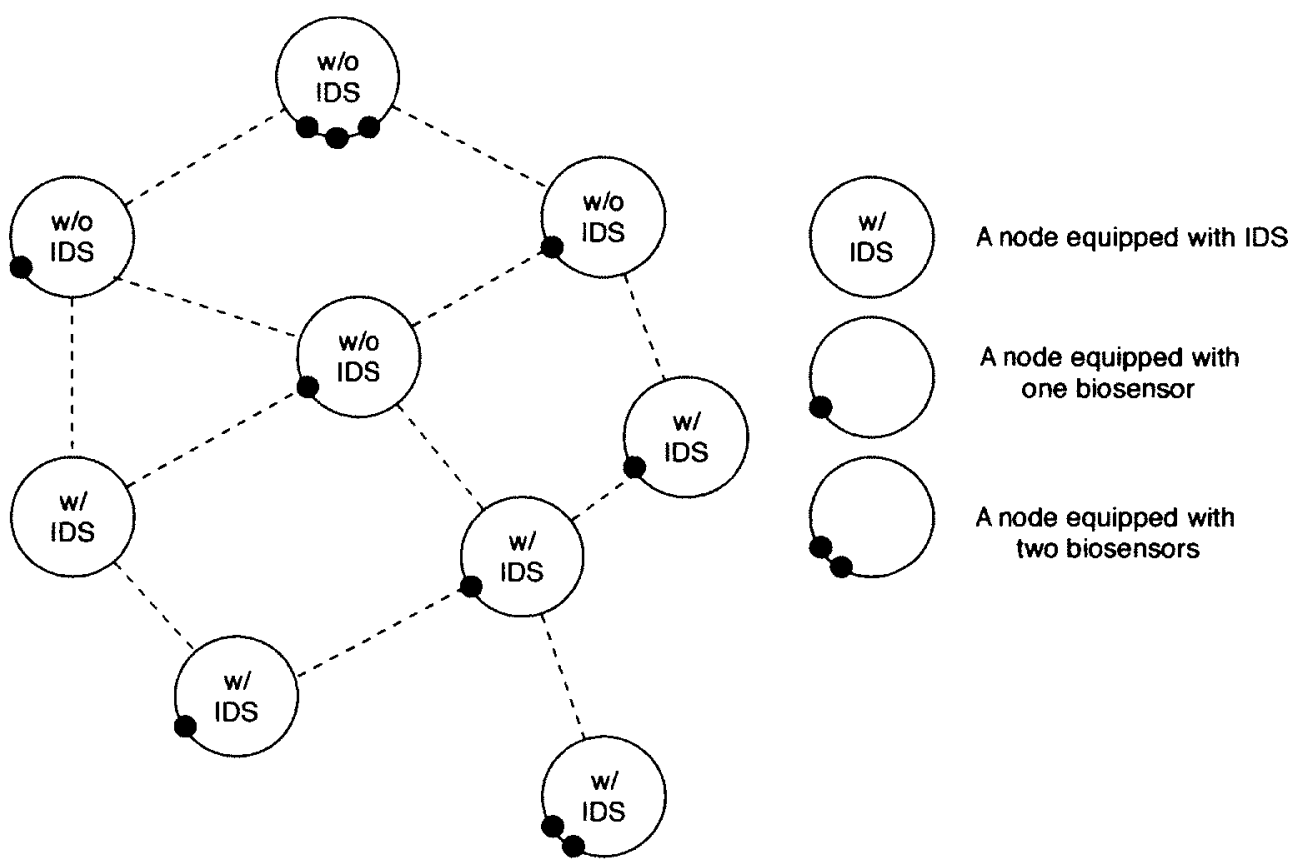

Figure 3.1: An example framework for a MANET with biosensors and IDSs.

executed at every time instant as well. However, intrusion detection and authentication may consume a large amount of energy, which is a concern for energy-constrained devices in MANETs. Moreover, performing authentication and intrusion detection may lead to security information leakage to an adversary monitoring communications and network behavior. Therefore, it is critical for the system to optimally schedule the intrusion detection and authentication activities for each time slot, taking system security and energy into account.

In the proposed scheme, since each sensor (biosensor or IDS) has measurement and estimation limitation, more than one biosensor and IDS (assume $L$ devices) are chosen for detecting the security states of the system. Each sensor monitors its local environment, not other sensors'. Then, their observations can be fused to increase observation accuracy using Dempster-Shafer theory. The number of sensors chosen is determined by the required level of network performance.

In the proposed scheme, a Markov model is used. Let the state of an arbitrary 
sensor $n, n \in\{1,2, \ldots, N\}$, be $x^{(n)}(t)$ at time $t$, which includes the sensor security and energy states $\left[s^{(n)}(t), e^{(n)}(t)\right]$. Each state represents the security condition and the residual battery energy level of sensor $n$ at time $t$. For example, security state space $I$ can include two security levels, namely $\{$ safe,compromised $\}$. The residual battery energy of each sensor can be divided into $h$ discrete levels. Therefore, the residual energy state space $\mathcal{E}$ includes the energy states $\left\{e_{1}, \ldots, e_{h}\right\}$ [112]. For example, the residual energy state space $\mathcal{E}$ can include two energy levels, namely $\{$ High, Low $\}$.

We consider that the time axis is divided into slots with equal duration, which corresponds to the time interval between two continuous user authentications. The chosen length of each time slot depends on the security requirement and energy states of the system. For example, the time interval for the system used in a tactic environment could be shorter than that used in a safer environment.

Let $x_{k}^{(n)}, s_{k}^{(n)}$ and $e_{k}^{(n)}$ denote the state of sensor $n$, its security state, and its residual energy state, respectively, at discrete time $k=0,1, \ldots$ The states $s_{k}^{(n)}$ and $e_{k}^{(n)}$ evolve based on $I$-state and $\mathcal{E}$-state Markov chains with transition probability matrix $U^{(n)}$ and $V^{(n)}$, respectively, if sensor $n$ is used at time $k$, which are described as follows:

$$
\begin{aligned}
& U^{(n)}=\left(\phi_{i j}^{(n)}\right)_{i, j \in I}, \text { where } \phi_{i j}^{(n)}=P\left(s_{k+1}^{(n)}=j \mid s_{k}^{(n)}=i\right) . \\
& V^{(n)}=\left(\psi_{i j}^{(n)}\right)_{i, j \in \mathcal{E}}, \text { where } \psi_{i j}^{(n)}=P\left(e_{k+1}^{(n)}=j \mid e_{k}^{(n)}=i\right) .
\end{aligned}
$$

The states of other idle sensors are unchanged, i.e., $s_{k+1}^{(n)}=s_{k}^{(n)}$ and $e_{k+1}^{(n)}=e_{k}^{(n)}$, if sensor $n$ is idle at time $k$. Hence, the state of sensor $n$ transitions with probability matrix $T^{(n)}=\left[U^{(n)} \otimes V^{(n)}\right]$, where $\otimes$ denotes the Kronecker product. For example, if there are two security states and two energy states, the state transition probability 


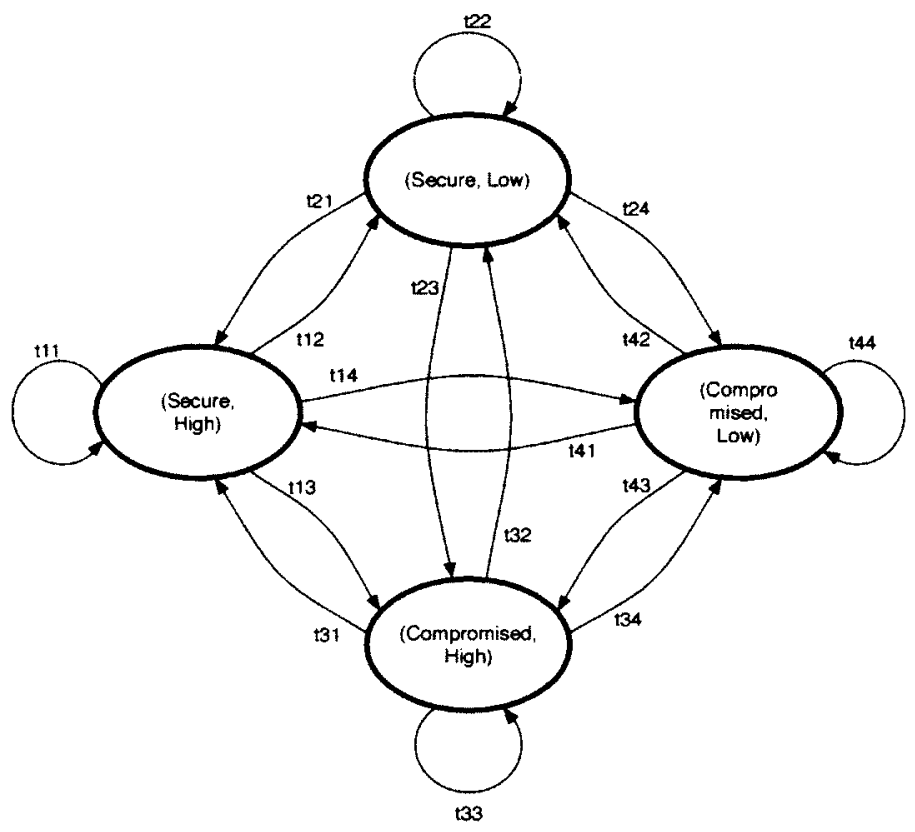

Figure 3.2: An example of Markov chain for a single node's state transition.

matrix of each sensor is a $4 * 4$ matrix, whose Markov chain is illustrated in Fig. 3.2.

Security-related and energy-related costs are considered in our scheme, since transmitted biometric information may be detected by adversaries ${ }^{1}$, and energy is certainly consumed when a sensor is used. For example, when cryptographically encoded data (i.e., using public key infrastructure) are sent from biometric sensors to other parts of the biometric systems, an adversary can perform a replay attack: the adversary intercepts the transmitted encrypted data when a genuine user is interacting with the system. The adversary then sends the captured data to the desired biometric parts whenever he wants to break into the system [113].

Let $a_{k} \in\{1, \ldots, N\}$ denote one chosen sensor at time $k$. Its corresponding information leakage cost at time $k$ is defined as $c_{s}\left(s_{k}^{\left(a_{k}\right)}, a_{k}\right)$, which is a function of the security state of the chosen sensor and action at that time. The corresponding energy

\footnotetext{
${ }^{1}$ Even if this information is encrypted, the fact that it is being transmitted conveys information to an adversary.
} 
cost at time $k$ is defined as $c_{e}\left(e_{k}^{\left(a_{k}\right)}, a_{k}\right)$, which is a function of the energy state of the chosen sensor and action at that time. If sensor $n$ is used at time $k$, an instantaneous cost $\beta^{k} c\left(x_{k}^{(n)}, n\right)$ is accrued, where $c\left(x_{k}^{(n)}, n\right)=(1-\lambda) c_{s}\left(s_{k}^{(n)}, n\right)+\lambda c_{e}\left(e_{k}^{(n)}, n\right)$, where $\lambda \in(0,1)$ is the weight factor for these two kinds of costs, which can be set differently in different applications. For example, in a battlefield MANET, the weight factor $\lambda$ can be set to a value close to 0 , which reflects the fact that information leakage is more important than energy loss in the battlefield network. By contrast, in a civilian MANET, $\lambda$ can be set to a larger value, which reflects the fact that energy loss is more important than information leakage in the civilian network. $\beta$ $(0 \leq \beta<1)$ denotes the discount factor, which is needed to make the infinite sum converge mathematically in $(3.4)[114,115]$. The discount factor can model the fact that future cost is worth less than immediate cost because the future is less certain. In the proposed scheme, the total instantaneous cost $C_{k}$ at time $k$ is determined as follows: define a set $S L \subseteq\{1, \ldots, N\},|S L|=L$.

$$
C_{k}=\beta^{k} \times \sum_{n=1}^{L} c\left(x_{k}^{(n)}, n\right), n \in S L
$$

The total expected discounted cost over an infinite-time horizon is given by

$$
J_{\mu}=E\left[\sum_{k=0}^{\infty} C_{k}\right]
$$

The optimization objective is to find the optimal stationary policy $\mu^{*}=\arg \min _{\mu \in \eta} J_{\mu}$ to minimize the cost in (3.4). 


\subsubsection{Data Fusion of Biometric Sensors and IDSs}

In the proposed scheme, $L$ sensors are chosen for authentication and intrusion detection at each time slot to observe the security state of the network (How to optimally schedule the $L$ sensors is presented in Section 3.2.3). In order to obtain the security state of the network, these observation values are combined and a decision about the security state of the network is made. However, since there is some probability that a given sensor might either be in a compromised state, or have made an inaccurate assessment, it is possible that this sensor has contributed an unreliable observation. It can be quite difficult to ascertain which observers are compromised. Therefore, choosing an appropriate fusion method is critical for the proposed scheme.

Existing fusion methods can be classified as follows based on the output information level of the base classifiers [116]: Type I classifiers output single class labels (SCL). Majority voting and behavior-knowledge space are two most representative methods for fusing SCL classifiers. Majority voting can operate under the assumption that most of the observing nodes are trustworthy. Type II classifiers output class rankings. Two major fusion methods of type II classifiers' outputs are based on either a class set reduction (CSR) or a class set reordering (CSRR). CSR methods try to find the minimal reduced class set, in which the true class is still represented. CSRR methods try to increase the true class ranking as high as possible. Type III classifiers produce so-called soft outputs, which are real values in the range $[0,1]$. Fusion methods for type III classifiers try to reduce the uncertain level and maximize suitable measurements of evidence. Fusion methods include Bayesian fusion methods, fuzzy integrals, Dempster-Shafer combination, fuzzy templates, product of experts and artificial neural networks.

The Dempster-Shafer evidence theory was originated by Arthur Dempster and later revised by Glenn Shafer. Its essential idea is that an observer can obtain degrees 
of belief about a proposition from a related proposition's subjective probabilities. The motivation for selecting Dempster-Shafer theory to solve the fusion problem in our proposed scheme is as follows [107]:

- It has a relatively high degree of theoretical development for handling uncertainty or ignorance.

- It provides a convenient numerical procedure for combining disparate data obtained from multiple sources.

- It is widely used in various applications.

In a Dempster-Shafer reasoning system, a set of mutually exclusive and exhaustive possibilities are enumerated in the frame of discernment, denoted by $\Omega$ [117]. In this section, two security states for each node, $\{$ secure, compromised $\}$, are used to demonstrate how to use Dempster-Shafer theory in the fusion of biometric sensors and IDSs. Note that the theory can be applied for nodes with more than two security states. In the proposed scheme, the frame of discernment consists of two possibilities concerning the security state of an arbitrary node $a$, namely, $\Omega=$ secure,compromised $\}$, which represents that node $a$ has two security states: secure state and compromised state. Any hypothesis $H$ refers to a subset of $\Omega$ for which the neighboring biometric sensors and IDSs can present evidence. The set of all possible subsets of $\Omega$, including itself and the null set, is called a power set and designated as $2^{\Omega}$ [117]. For $\Omega$ in the proposed scheme, the power set has three focal elements, which are the three non-null subsets: hypothesis $H=\{$ secure $\}$, hypothesis $\bar{H}=\{$ compromised $\}$, and hypothesis $U=\Omega$, which means that the observed sensor $a$ is either in the secure state or the compromised state. Each biometric sensor and IDS contributes its observation by assigning its beliefs over $\Omega$. 
If a sensor is trustworthy, then the sensor always provides accurate observation data. Any chosen node could be untrustworthy due to its current compromised state or inaccurate detection. The chosen node $n$ is trustworthy for an arbitrary observed node $a$ at the time slot $k+1$ when it is in the secure state, and detects accurately. The trustworthy probability $t p_{k+1}^{(n)}$ of node $n$ at time $k+1$ is equal to $P\left(s_{k+1}^{(n)}=\right.$ secure $) \times P\left(y_{k+1}^{(n)}=s_{k+1}^{(a)}\right)$, where $y_{k+1}^{(n)}$ is the observation of $a$ 's security state obtained from node $n$. In our scheme, $P\left(y_{k+1}^{(n)}=\right.$ secure $\mid s_{k+1}^{(a)}=$ secure $)$ and $P\left(y_{k+1}^{(n)}=\right.$ compromised $\mid s_{k+1}^{(a)}=$ compromised $)$ are equal to $1-F A R$ and $1-F R R$, respectively. $F A R$ and $F R R$ are the frequency of false acceptance errors and of false rejection errors for node $n$, respectively. Otherwise, node $n$ is not trustworthy with probability $1-t p_{k+1}^{(n)}$. Suppose that node $n$ states that node $a$ is secure. If node $n$ is trustworthy, then its claim is accurate. If $n$ is not trustworthy, its claim is not necessarily inaccurate.

Basic probability assignment reflects the evidence's strength of support [117]. For example, for node $n$, the basic probability number $m_{n}(H)$ is defined as the portion of total belief assigned to hypothesis $H$ [117]. When $n^{\prime} s$ observation data $y_{k+1}^{(n)}$ for the security state of node $a$ at time $k+1$ is equal to secure, its basic probability assignment can be calculated as follows [117]:

$$
\begin{aligned}
& m_{n}(H)=P\left(s_{k+1}^{(n)}=\text { secure }\right) \times P\left(y_{k+1}^{(n)}=\text { secure } \mid s_{k+1}^{(a)}=\text { secure }\right) \\
& m_{n}(\bar{H})=P\left(s_{k+1}^{(n)}=\text { secure }\right) \times P\left(y_{k+1}^{(n)}=\text { secure } \mid s_{k+1}^{(a)}=\text { compromised }\right) \\
& m_{n}(U)=P\left(s_{k+1}^{(n)}=\text { compromised }\right) .
\end{aligned}
$$

If node $n$ claims that node $a$ is compromised, its basic probability assignment can be 
calculated as follows [117]:

$$
\begin{aligned}
& m_{n}(H)=P\left(s_{k+1}^{(n)}=\text { secure }\right) \times P\left(y_{k+1}^{(n)}=\text { compromised } \mid s_{k+1}^{(a)}=\text { secure }\right) \\
& m_{n}(\bar{H})=P\left(s_{k+1}^{(n)}=\text { secure }\right) \times P\left(y_{k+1}^{(n)}=\text { compromised } \mid s_{k+1}^{(a)}=\text { compromised }\right), \\
& m_{n}(U)=P\left(s_{k+1}^{(n)}=\text { compromised }\right) .
\end{aligned}
$$

In the remainder of this section, two biometric sensors $b$ and $c$ are used to demonstrate how to apply Dempster-Shafer theory in combining the belief $m_{b}$ of sensor $b$ and the belief $m_{c}$ of sensor $c$ in the hypotheses. When these two biometric sensors have the same accuracy estimations or they are in situations where their probability assignments over the frame of discernment can quantitatively reflect the ignorance of each other's observations, the equally trusting approach is used in Dempster-Shafer evidence combination [117]. The combined belief of sensors $b$ and $c$ can be calculated as follows [117]:

$$
\begin{aligned}
m_{b}(H) \oplus m_{c}(H) & =\frac{1}{K}\left[m_{b}(H) m_{c}(H)+m_{b}(H) m_{c}(U)+m_{b}(U) m_{c}(H)\right] \\
m_{b}(\bar{H}) \oplus m_{c}(\bar{H}) & =\frac{1}{K}\left[m_{b}(\bar{H}) m_{c}(\bar{H})+m_{b}(\bar{H}) m_{c}(U)+m_{b}(U) m_{c}(\bar{H})\right] \\
m_{b}(U) \oplus m_{c}(U) & =\frac{1}{K} m_{b}(U) m_{c}(U)
\end{aligned}
$$

where

$$
\begin{aligned}
K= & m_{b}(H) m_{c}(H)+m_{b}(H) m_{c}(U)+m_{b}(U) m_{c}(U)+m_{b}(U) m_{c}(H)+ \\
& m_{b}(U) m_{c}(\bar{H})+m_{b}(\bar{H}) m_{c}(\bar{H})+m_{b}(\bar{H}) m_{c}(U) .
\end{aligned}
$$

If these biometric sensors observe with different accuracy, the weighted DempsterShafer evidence combining rule is used in Dempster-Shafer evidence combination 
[107]. Based on the historical performances of the sensors in similar situations, their corresponding correctness rates are used as the references to decide how much the sensors' current estimations should be trusted from their current observation. Let $w_{b}$ and $w_{c}$ be the corresponding estimation correctness rates in history for $b$ and $c$, respectively. Then, the combined belief of biometric sensors $b$ and $c$ can be calculated as follows [107]:

$$
\begin{aligned}
m_{b}(H) \oplus m_{c}(H)= & \frac{1}{K}\left[w_{b} m_{b}(H) w_{c} m_{c}(H)+w_{b} m_{b}(H) w_{c} m_{c}(U)\right. \\
& \left.+w_{b} m_{b}(U) w_{c} m_{c}(H)\right], \\
m_{b}(\bar{H}) \oplus m_{c}(\bar{H})= & \frac{1}{K}\left[w_{b} m_{b}(\bar{H}) w_{c} m_{c}(\bar{H})+w_{b} m_{b}(\bar{H}) w_{c} m_{c}(U)\right. \\
& \left.+w_{b} m_{b}(U) w_{c} m_{c}(\bar{H})\right], \\
m_{b}(U) \oplus m_{c}(U)= & \frac{1}{K} w_{b} m_{b}(U) w_{c} m_{c}(U),
\end{aligned}
$$

where

$$
K=1-w_{a} w_{b}\left[m_{a}(H) m_{b}(\bar{H})+m_{a}(\bar{H}) m_{b}(H)\right] .
$$

If more than two sensors are chosen at each time slot, the evidence can be computed by combining any pair of arguments and then combining the results with the remaining arguments. Since inaccurate detection is the main characteristic of untrustworthy sensors, only detection errors are considered in the proposed scheme.

\subsubsection{Formulation of the Distributed User Authentication and Intrusion Detection Scheduling Problem}

In this section, we formulate the distributed authentication and intrusion detection scheduling problem as a POMDP multi-armed bandit problem $[8,114]$. 


\subsubsection{Information State Formulation}

The decision about which sensors are chosen should not totally depend on the current observation values, since the sensors' states are only partially observable. Therefore, all the actions and observations in the history should be counted as a basis for decision-making under environmental uncertainties. To this end, information state is developed to derive sufficient statistical information for the past history, including all the actions and observations. The information state of a sensor refers to a probability distribution over the sensor's states. The entire probability space (the set of all possible probability distributions) is referred to as the information space.

For an arbitrary sensor $n$, the information state at time slot $k$ is denoted as $\pi_{k}^{(n)}$ :

$$
\begin{array}{r}
\pi_{k}^{(n)}=\left(\pi_{k}^{(n)}(i)\right), i=1, \ldots, \zeta_{n} \\
\text { where } \pi_{k}^{(n)}(i)=P\left(x_{k}^{(n)}=i \mid Y_{k}, A_{k-1}\right), \text { and } 1_{\zeta_{n}}^{\prime} \pi^{(n)}=1 .
\end{array}
$$

In the above equation, $Y_{k}=\left(y_{1}^{\left(a_{0}\right)}, \ldots, y_{k}^{\left(a_{k-1}\right)}\right)$ and $A_{k-1}=\left(a_{0}, \ldots, a_{k-1}\right)$ denote the observation and action (sensor selection) history, respectively, at time $k .1_{\zeta_{n}}$ is an $\zeta_{n}$-dimensional column vector of ones.

If sensor $n$ is chosen at time $k$, a new observation $y_{k+1}^{(n)}$ is obtained at time $k+1$. Furthermore, an essential part of information state is that it can be updated after each corresponding action in order to incorporate one additional step of information into the history. Specifically, the information state at that time $\pi_{k+1}^{(n)}$ can be recursively updated by the hidden Markov model (HMM) state filter known as the forward algorithm with the new observation [8]:

$$
\pi_{k+1}^{(n)}=\frac{B^{(n)}\left(y_{k+1}^{(n)}\right) T^{(n)^{\prime}} \pi_{k}^{(n)}}{1_{\zeta_{n}}^{\prime} B^{(n)}\left(y_{k+1}^{(n)}\right) T^{(n)^{\prime}} \pi_{k}^{(n)}}
$$


where $B^{(n)}$ denotes the observation probability matrix of node $n$, which denotes the probabilities of the observation $y_{k+1}^{(n)}$ acquired when the sensor $n$ is picked at time $k+1$ given each state of the Markov chain. When $L$ nodes are used, the observation probability matrix is obtained by using the above Dempster-Shafer evidence combining rules. Since the other $N-L$ sensors are not used at time $k$, their information states remain unchanged at time $k+1$.

The above POMDP multi-armed bandit problem can be re-expressed as a fully observable multi-armed bandit problem in terms of the information state, which means optimal sensors can be chosen based on the information state [115]. This is because although the state of each sensor is not totally observable, its information state space is totally observable. Generally, we need to solve N-POMDP with large computational complexity. However, it is proved that an indexable property exists that can dramatically simplify the computation and implementation of the optimal policy, meaning that the optimal policy can be found according to the Gittins indices [115] of the sensors. Sensors' Gittins indices $\gamma^{(n)}\left(\pi_{k}^{(n)}\right)(n=1, \ldots, N)$ are used to choose the appropriate sensors at time $k$ [115]. Therefore, the optimal policy at time $k$ is that the $L$ sensors with the largest Gittins indices at that time should be selected. The above problem can be transformed to compute the Gittins index of each sensor. A finite-dimensional value iteration algorithm proposed in [8] is used to compute the Gittins index for each sensor.

\subsubsection{Distributed Scheduling Process}

To reduce the computational complexity of the proposed scheme, the distributed multimodal biometrics authentication and intrusion detection scheduling process can be divided into off-line and on-line parts.

a). Off-line Computation of Gittins Index 
As with any dynamic programming formulation, the computation of the Gittins index for each sensor can be done off-line. For an arbitrary sensor $n$, a set of vectors $\Lambda_{k}^{(n)}$ at each iteration $k$ are computed in advance based on the following parameters: state transition probability matrix $T^{(n)}$, observation probability matrix $B^{(n)}$, reward vector $R^{(n)}$, initial information state $\pi_{0}^{(n)}$, horizon length $H$, and discount factor $\beta$.

b). Real-time Sensor Selection over Horizon $H$

At time $k$, each sensor stores the sensors current Gittins indices into an $N$ dimensional vector. The real-time sensor selection includes the following steps:

1. Select $L$ sensors with the highest Gittins indices at time $k$. For these $L$ sensors, perform steps 1 to 5 .

2. Get new sensor observations $y_{k+1}^{(n)}$ at time $k+1$.

3. Update the information states of the $L$ chosen sensors using the corresponding HMM filters.

4. Compute the Gittins index $\gamma_{H}^{(n)}\left(\pi_{k+1}^{(n)}\right)$ for each of these $L$ sensors only.

5. Broadcast the new Gittins indices to the other sensors.

6. On receiving the messages, all the sensors update their Gittins indices. Go to step 1.

\subsubsection{Discussion of Computational Complexity and Communication Overhead}

In the proposed scheme, the optimal policy can be found by a Gittins index rule, which means that the scheduling problem only needs to solve the individual POMDPs for each sensor. Therefore, the computational complexity of the proposed scheme is dramatically decreased. For the on-line real time scheduling of different sensors, each 
sensor just looks up the pre-built index table to find the index value corresponding to the current state. A look-up table can be designed with little computational complexity. In addition, several computationally efficient algorithms can be found in [118] to further reduce the computational complexity of the proposed scheme. For example, based on Lovejoy's suboptimal algorithm, the value function can be upper and lower bounded, and efficient suboptimal solutions can be developed [119].

In the proposed scheme, communication overhead is mainly due to multicasting the following two types of messages in the real-time scheduling process:

- INTIAL-SENSOR-INDICES (ISIND), 8 bytes, which is sent at the beginning of the authentication and intrusion detection process, so that each sensor knows the others' Gittins indices.

- SENSOR-INDICES (SIND), 8 bytes, which is sent at the beginning of each time slot by the $L$ nodes active in the previous time slot.

Any network layer multicast algorithm for ad-hoc networks can be used in the scheme. The proposed scheme's total communication overhead is proportional to $8 N \times(N-$ 1) bytes, plus $8 L \times(N-1)$ bytes per time slot. Overall, the proposed scheme's communication overhead is similar to that of the centralized scheme, as they are both bounded by $O(L N)$.

\subsubsection{Simulation Results and Discussions}

In this section, we use computer simulations to evaluate the performance of the proposed scheme with and without using data fusion. We consider the following simulation scenario. A MANET is equipped with two biosensors for continuous authentication, iris sensor and fingerprint sensor. Each sensor includes two security states, safe and compromised, and two energy states, high and low, which means that there are 
4 states for each sensor. The iris sensor is the more expensive, and also provides the more accurate authentication. The fingerprint sensor provides intermediate security authentication, and has intermediate energy cost. There is an IDS in the MANET, which uses the least energy, and has the least accuracy in detecting the security state. The following defined matrices are based on the above assumptions.

The security state transition matrices of the iris sensor, fingerprint sensor and IDS, when they are active, are defined as follows:

$$
U^{(1)}=\left(\begin{array}{cc}
0.95 & 0.05 \\
0.30 & 0.70
\end{array}\right), U^{(2)}=\left(\begin{array}{cc}
0.80 & 0.20 \\
0.10 & 0.90
\end{array}\right), U^{(3)}=\left(\begin{array}{ll}
0.98 & 0.02 \\
0.02 & 0.98
\end{array}\right)
$$

From these state transition matrices, we can see the probability of changing from one state to another. For example, the iris sensor could be compromised with probability 0.05 , and retrieved back to the safe state with probability 0.30 . The corresponding energy state transition matrices are defined as:

$$
V^{(1)}=\left(\begin{array}{cc}
0.96 & 0.04 \\
0 & 1
\end{array}\right), V^{(2)}=\left(\begin{array}{cc}
0.98 & 0.02 \\
0 & 1
\end{array}\right), V^{(3)}=\left(\begin{array}{cc}
0.99 & 0.01 \\
0 & 1
\end{array}\right) .
$$

Which means that when the battery residual energy is low, it cannot transition back to the high-energy level. The observation matrices for the security state and energy state are respectively defined as:

$$
B_{s}^{(1)}=\left(\begin{array}{ll}
0.97 & 0.03 \\
0.03 & 0.97
\end{array}\right), B_{s}^{(2)}=\left(\begin{array}{cc}
0.90 & 0.10 \\
0.10 & 0.90
\end{array}\right), B_{s}^{(3)}=\left(\begin{array}{ll}
0.80 & 0.20 \\
0.20 & 0.80
\end{array}\right), B_{e}=
$$

$\left(\begin{array}{cc}0.90 & 0.10 \\ 0.10 & 0.90\end{array}\right)$

The cost matrices are defined as: $C(1)=(3,8,25,27), C(2)=(2,7,20,26), C(3)=$ $(1,4,27,31)$. Since there is more potential for information leakage when a node is in 
the compromised state than in the safe state, the information leakage cost of selecting a safe node is lower than that of selecting a compromised node ${ }^{2}$. When the network is compromised, an extra cost $C_{\text {net }}$ is applied into the total cost. In our simulation, $C_{n e t}$ is set to 30 based on the nodes' cost matrices, although different values could be chosen without affecting the results of our comparisons.

It is a non-trivial task to setup transition matrices and cost matrices for the proposed scheme. We assume that most nodes' properties can be made known when constructing these matrices, which should be realistic particularly for tactical MANETs where initial device management and planning is an a priori requirement. By "node properties" we mean the information and states that are used as inputs to the transition and cost matrices. However, in a dynamic environment, where heterogeneous nodes may join the network, it may not be realistic to assume knowledge of all nodes' properties. In these circumstances, we should be able to predict and learn the nodes' properties from the history of observations and actions.

We used 'pomdp-solve', a program in $\mathrm{C}++$ from [120], to compute the set of vectors $\wedge_{H}$ required for the algorithm in [8]. In pomdp-solve, we chose the incremental pruning algorithm developed in the artificial intelligence community by Cassandra et al. [118], since it is one of the fastest algorithms for solving POMDPs [8]. We implemented the computation of the Gittins indices in Matlab. All simulations are run and policy vectors computed on a computer equipped with Windows 7 , Intel Core 2 Duo P8400 CPU (2.26Ghz), 4GB memory. In the simulations, the initial state for each node is high-energy and secure. We adopt the hybrid Manhattan and RWP mobility model [121] to simulate the nodes moving. Block Rayleigh flat-fading wireless channel model [122] is used.

\footnotetext{
${ }^{2}$ For example, a compromised node could intentionally introduce collisions in a cryptographic protocol.
} 


\subsubsection{Performance Improvement}

We run simulations to compare the cost of three approaches: the proposed scheme with data fusion, the proposed scheme without data fusion and an existing scheme that does not consider optimal scheduling (that is, a scheme that makes selections randomly). Each cost value is the averaged result of 10000 simulations. Fig. 3.3 illustrates the average cost for the first 100 steps (i.e., time slots) of the simulation. Fig. 3.4 illustrates the relative information leakage, which is defined as the information leakage of the selected nodes divided by the information leakage when the nodes are in the worst state. The results show that the proposed scheme with data fusion and the proposed scheme without data fusion have lower cost and less information leakage than the existing scheme. Thus through optimal node selection, the system can be more secure and energy-efficient. From these figures, we can observe that data fusion can improve performance. The reason why the fusion scheme has better performance is that data fusion using Dempster-Shafer theory increases the observation accuracy by combining the observations from multiple sensors.

Various transition probabilities are also used in the simulations to evaluate the dynamic stability of the proposed scheme. Fig. 3.5 shows the average cost of these three schemes when the first component in the security transition probability matrix varies from 0.7 to 1.0 , where high transition probability means that the system is more secure. The results still show that the proposed scheme with data fusion has the lowest cost among these three schemes. From Fig. 3.5, we can also see that the cost decreases when the system becomes more secure. This is because the information leakage is smaller when the system becomes more secure, therefore the cost is smaller. When the security transition probability reaches 1 , the proposed schemes lose their advantages since all of the nodes always stay in the secure states - that is, there is no added value in trying to optimize security in an infallible network. Fig. 3.6 shows the 


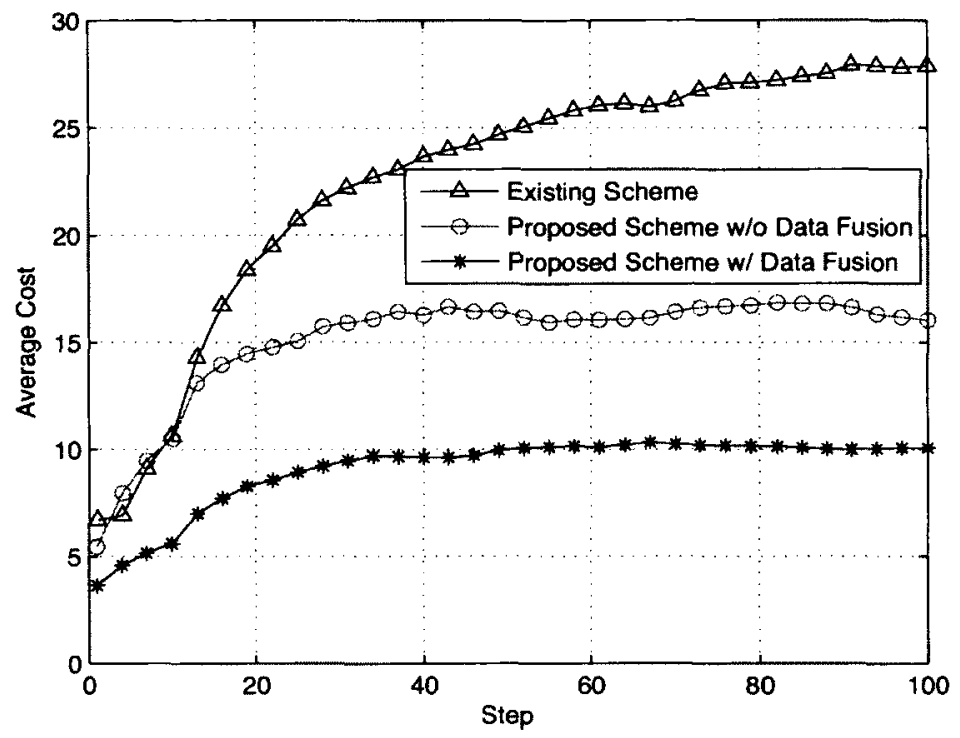

Figure 3.3: Cost comparison among the proposed scheme with data fusion, the proposed scheme without data fusion and the existing scheme.

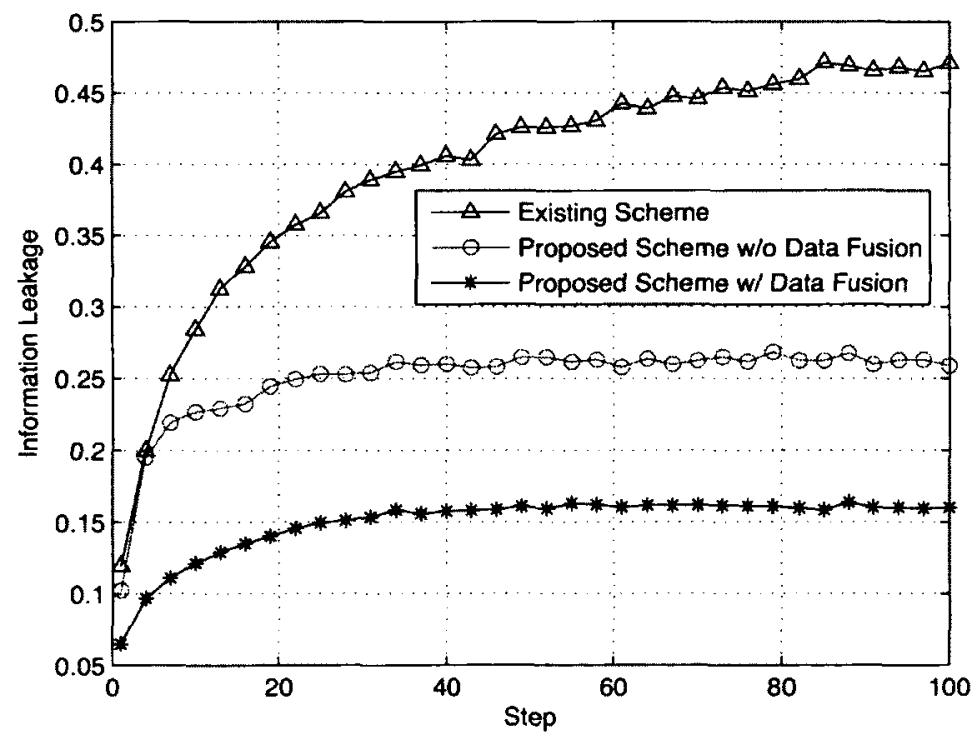

Figure 3.4: Information leakage comparison among three schemes. 


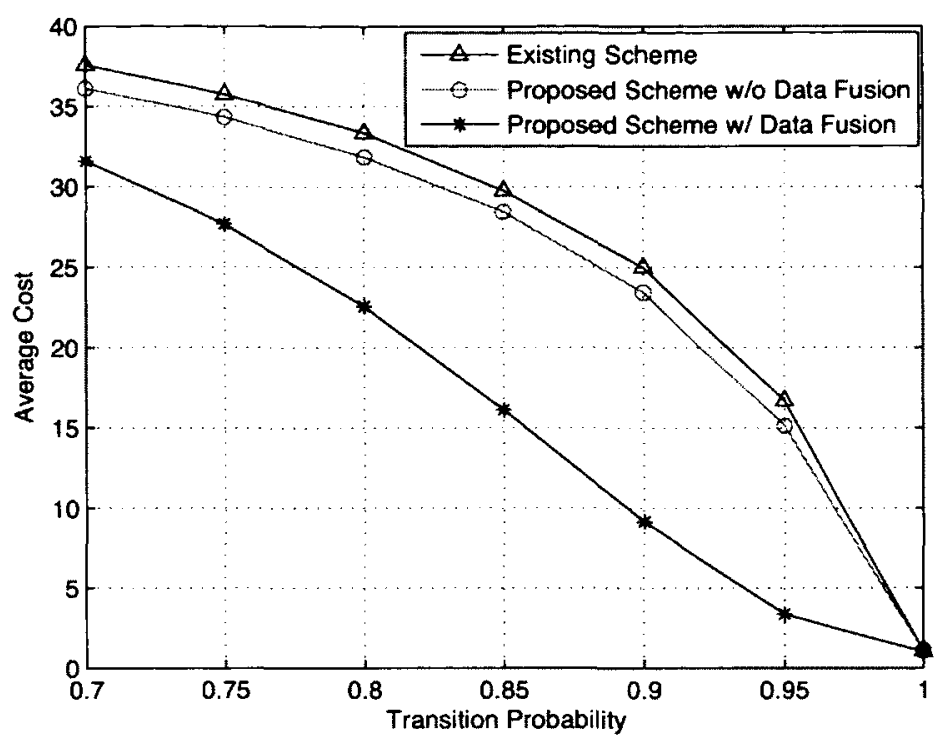

Figure 3.5: Cost comparison among three schemes under different transition probabilities.

average information leakage within the first 40 steps when the first component in the security transition probability matrix of the IDS varies from 0.65 to 0.95 and all other probability values remain constant. The results show the information leakage of the proposed schemes remain low and stable. The reason for this is that the proposed schemes avoid choosing the compromised nodes, and therefore decrease information leakage and network compromise probability.

Different numbers of nodes are also used in the simulations to verify the scalability of the proposed scheme. Fig. 3.7 shows the average cost within the first 100 steps of the simulations of networks of different sizes. In these simulations, we use the same three kinds of nodes mentioned earlier. The results show that the proposed scheme with data fusion has the lowest cost compared to the existing scheme and the proposed scheme without data fusion. The results also show that the cost of the proposed schemes and existing scheme decreases when the number of available nodes in the network increases from 3 to 18 . The reason is that there are more nodes that 


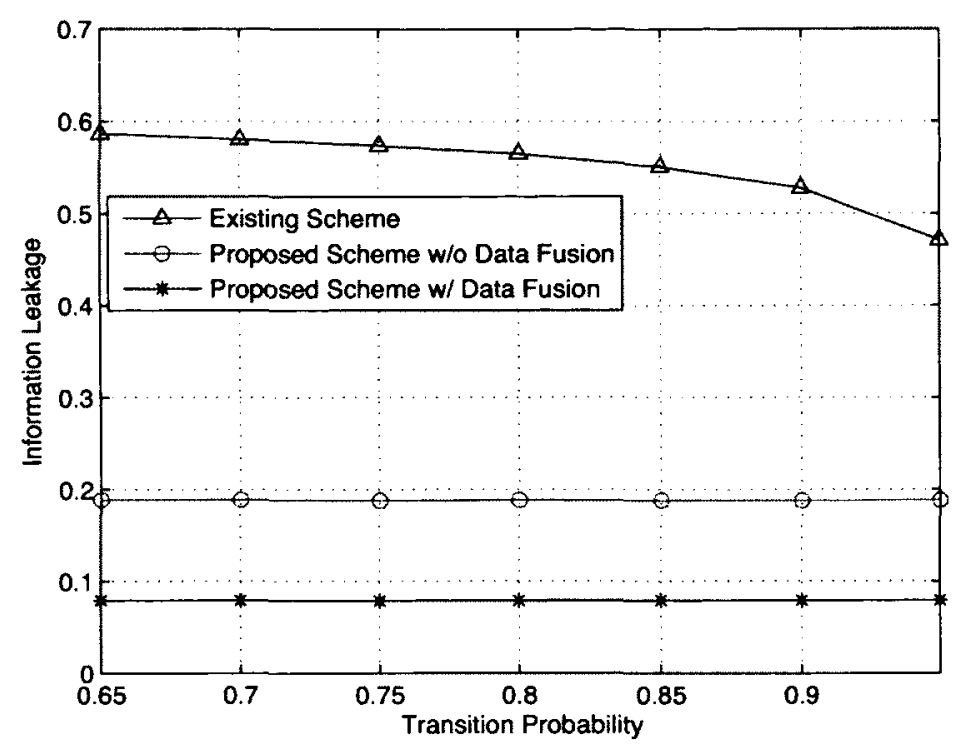

Figure 3.6: Information leakage comparison among three schemes under different transition probabilities.

can be selected for authentication and intrusion detection, so compromised nodes or low-energy nodes can be avoided. When the number of nodes increases to certain level, where most of nodes are in secure states, the average cost of the proposed schemes approaches the same low value.

\subsubsection{Network Compromise Probability Improvement}

In these simulations, we investigate the network compromise probability of the proposed scheme. In our simulations, the network is compromised when all of the chosen nodes are in the compromised states. For easy comparison of the network compromise probability in these three schemes, the energy transition probability of each node is set to 1 , so that the network dies from being compromised rather than energy exhaustion. Fig. 3.8 shows the average network compromised probability within the first 40 steps when the first component in the security transition probability matrix of the IDS varies from 0.65 to 0.95 and all other probability values remain constant. The 


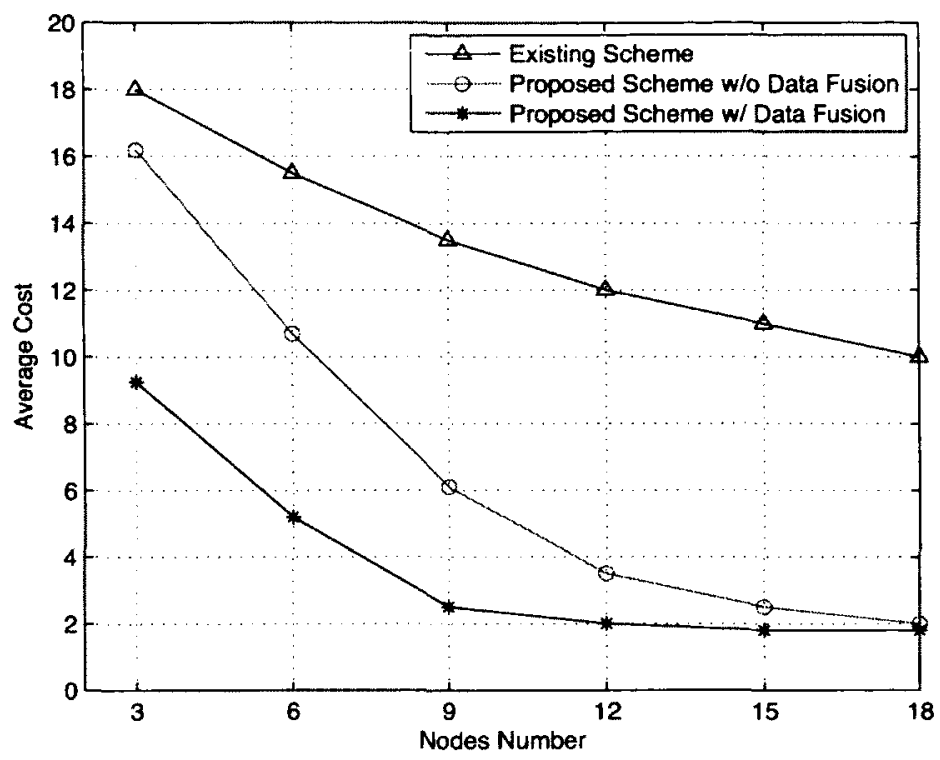

Figure 3.7: Cost comparison among three schemes with varying nodes in the network.

results show the network compromise probability of two proposed schemes keep low and stable. When the security transition probability is low, meaning that the IDS is easily compromised, the proposed schemes outperform the existing scheme by an even greater amount than the case when the IDS is more secure. The reason is that the proposed schemes avoid choosing the compromised nodes, and therefore decreasing the probability of the whole network becoming compromised.

\subsubsection{Network Lifetime Improvement and the Optimal Policy}

Network lifetime performance has been evaluated for the proposed scheme, which is illustrated in Fig. 3.9. Network lifetime is defined as the number of time slots until all of the chosen nodes in the network are in the low-energy state. In order to simplify our scheme, data fusion is not applied to the energy state of the nodes. For easy comparison of the network lifetime in different schemes, each node's security transition probability is set to 1 , so that the network dies from energy exhaustion in 


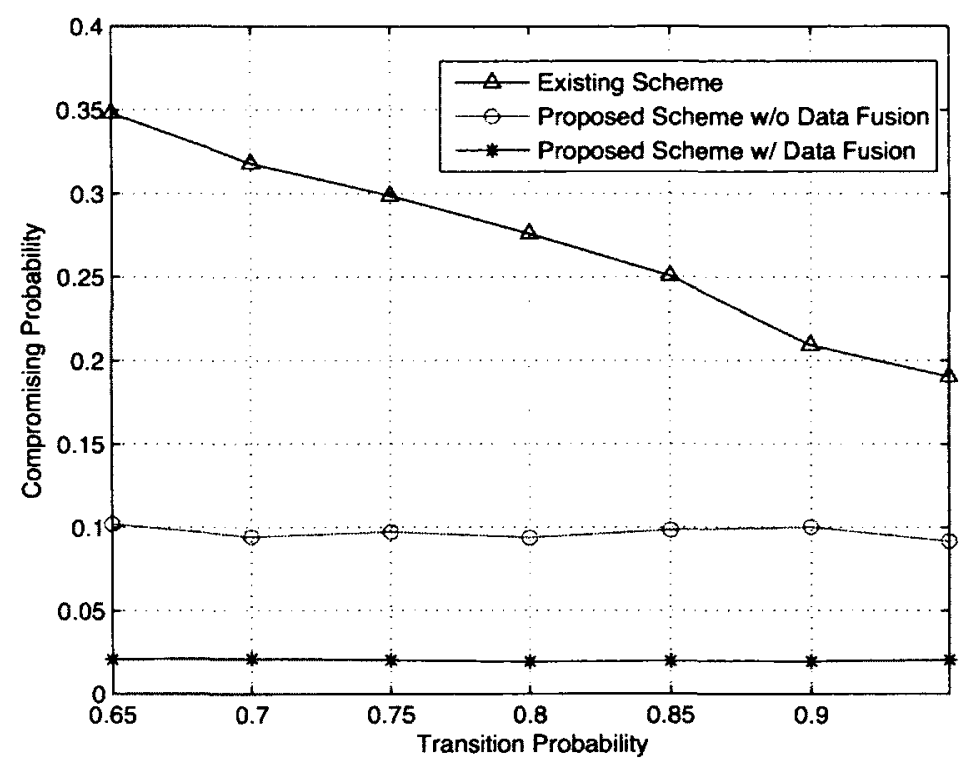

Figure 3.8: Network compromise comparison among three schemes under different transition probabilities.

all cases rather than becoming compromised.

We first check the performance when different energy transition probabilities are used. In the simulations, the first element in energy state transition probability matrix of the IDS varies from 0.65 to 0.9 , and all other probability values remain constant. Fig. 3.9 shows the proposed scheme has longer network lifetime than the existing scheme, since the proposed scheme selects the nodes based on their energy states, and only selects the expensive energy-consuming sensors when necessary. Hence, in the proposed scheme, the energy can be used more efficiently. In addition, as we can see from Fig. 3.9, the average network lifetime in the existing scheme is increased when the energy transition probability is increased. The reason is that, when the energy transition probability is increased, it is more likely that the system stays in high energy state. The results also show that the network lifetime of the proposed scheme remains high and stable. The network lifetime is also compared in Fig. 3.10, showing that the network lifetime increases with the total number of nodes, and 


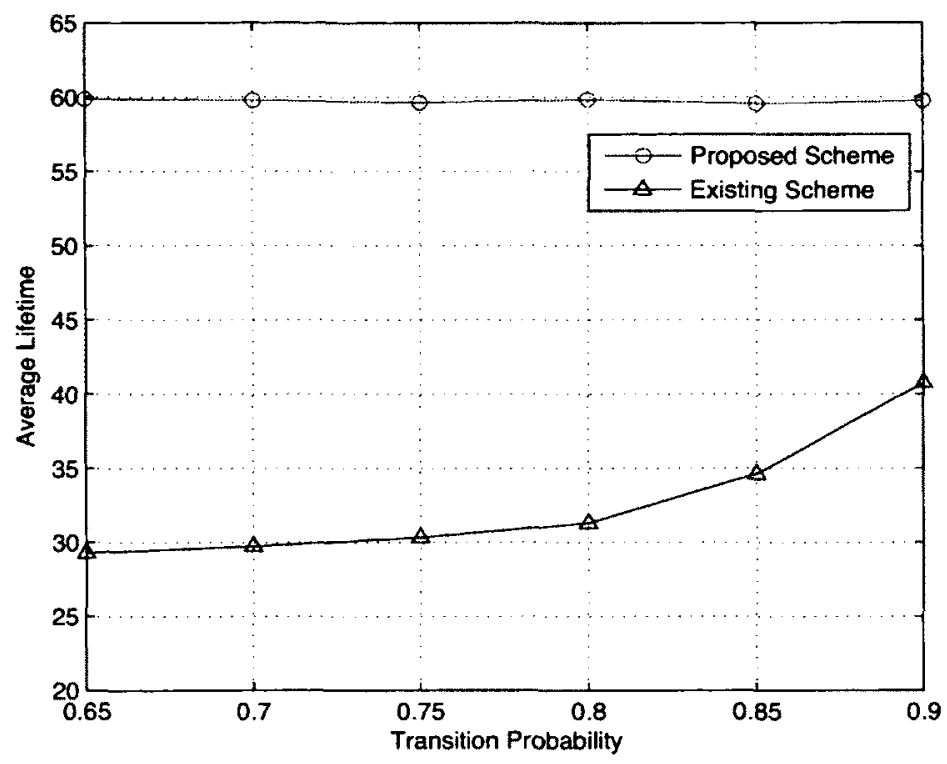

Figure 3.9: Network lifetime comparison between the proposed scheme and the existing scheme under different energy transition probabilities.

the proposed scheme show significant improvement over the existing scheme. The proposed scheme provides longer network lifetime through optimal scheduling.

Fig. 3.11 shows an example of the policy for optimal scheduling in the proposed scheme without data fusion. We can see that different sensors, iris, fingerprint, or IDS, are scheduled dynamically as the simulation runs to minimize the information leakage and maximize MANET's network lifetime.

\subsection{Structural Results for Combined Continuous User Authentication and Intrusion Detection}

In this section, we present a structural results method to calculate the Gittins index of each node in a large network with a variety of nodes, in order to further reduce computational complexity. 


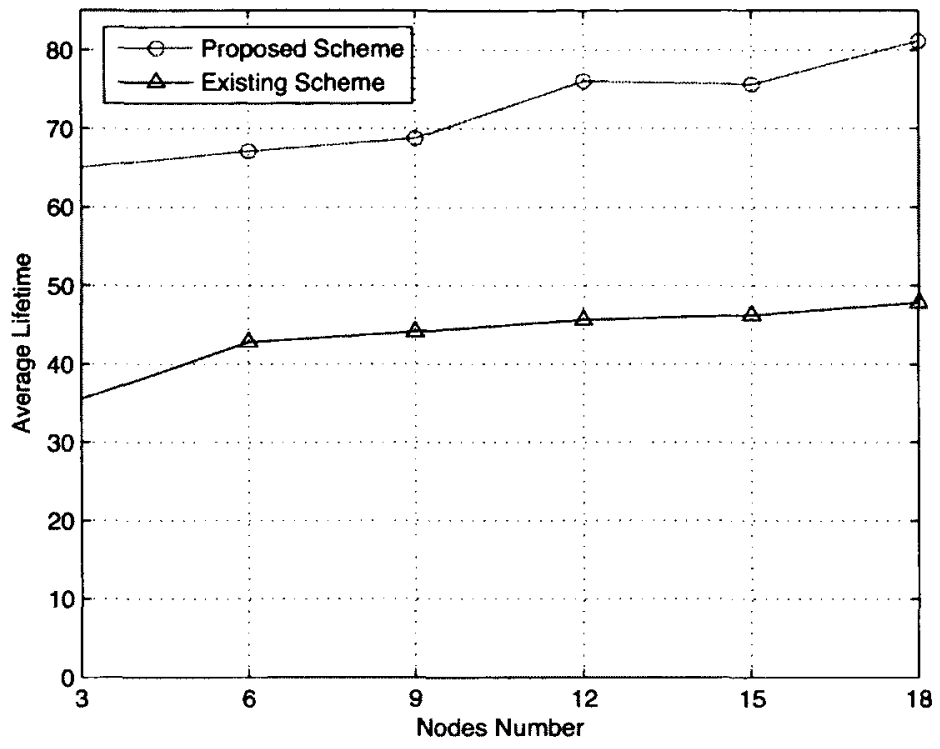

Figure 3.10: Network average lifetime with varying number of nodes.

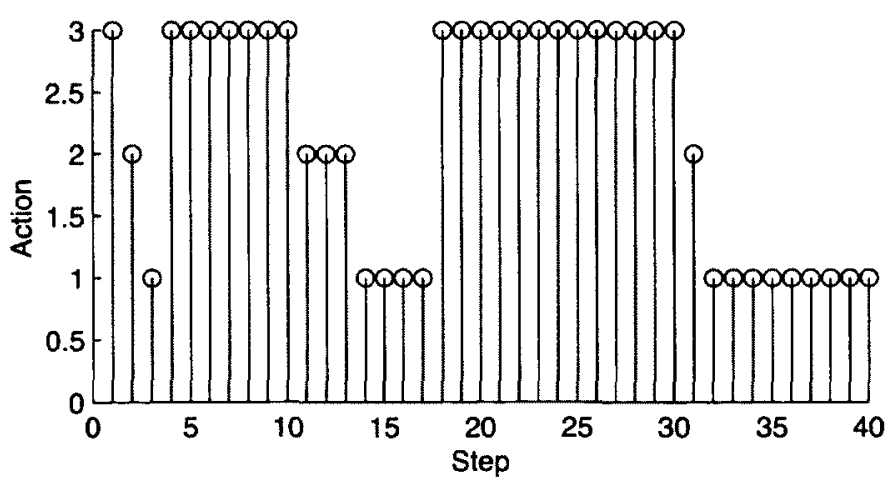

Figure 3.11: An example of the policy derived from the proposed scheme without data fusion. (Action $=1$ : The iris sensor is selected; Action $=2$ : The fingerprint sensor is selected; Action $=3$ : The IDS is selected) 


\subsubsection{Monotone Gittins Index in the Structural Results Method}

We show that, under reasonable conditions on the cost vector $C$, state transition probability matrix $T$ and observation probability matrix $B$ of each node in MANETs, the Gittins index in our problem can be monotone increasing in the information state (with respect to the monotone likelihood ratio (MLR) ordering [8]). This means that if the information states of these $N$ sensors at a given time instant are MLR comparable, the optimal policy is to pick the authentication sensor or the intrusion detection system with the smallest information state with respect to the MLR ordering. Namely, the sensor with the higher probability of being in the better state has a higher possibility of being chosen at that time slot, if the information states are MLR comparable. The definition of MLR ordering is described as follows.

\section{Definition 3.3.1 MLR Ordering.}

Assume that each sensor includes the same number of security and energy levels. Namely, $X_{1}, \ldots, X_{N}$ are equal to $X$.

1. Let $\pi_{1}$ and $\pi_{2}$ be two information state vectors. Then, $\pi_{1}$ is less than $\pi_{2}$ with respect to the $M L R$ ordering - denoted as $\pi_{1} \leq_{r} \pi_{2}$ if $\pi_{1}(i) \pi_{2}(j) \geq \pi_{2}(i) \pi_{1}(j), i<$ $j, i, j \in\{1, \ldots, X\}$. For example, $\pi_{1}=\left[\begin{array}{ll}0.3 & 0.20 .5\end{array}\right]$ and $\pi_{2}=\left[\begin{array}{ll}0.1 & 0.20 .7\end{array}\right]$, then $\pi_{1} \leq_{r} \pi_{2}$

2. A function $f(\cdot)$ is $M L R$ increasing if for all $\pi_{1}, \pi_{2} \in \Psi$ (information state space), $\pi_{1} \leq \pi_{2}$ implies $f\left(\pi_{1}\right) \leq f\left(\pi_{2}\right)$.

3. Let $\pi^{(1)}, \pi^{(2)}, \ldots, \pi^{(N)}$ denote the information states of $N$ sensors. Then they are said to be MLR comparable if for any $n, \widetilde{n} \in\{1, \ldots, N\}$, either $\pi^{(n)} \leq_{r} \pi^{(\widetilde{n})}$ or $\pi^{(n)} \geq_{r} \pi^{(\tilde{n})}$. 
4. Given MLR comparable information states of these $N$ sensors, denote the smallest information state (with respect to $M L R$ ordering) as $\min \left\{\pi^{(1)}, \ldots, \pi^{(N)}\right\}$ with index $\arg \min \left\{\pi^{(1)}, \ldots, \pi^{(N)}\right\}$.

In the following, we present the conditions on the parameters $C, T$ and $B$ of an arbitrary sensor, where its Gittins index $\gamma(\pi)$ is monotone in information state $\pi$ with respect to the MLR ordering.

Theorem 1 Consider the following assumptions for each sensor:

Assumption 1 Costs satisfy $C(i) \leq C(i+1)$.

Assumption 2 State transition probability matrix $T$ is totally positive of order 2 (TPQ), i.e., all its second order minors are non-negative. That is, determinants

$$
\left|\begin{array}{cc}
t_{i_{1} j_{1}} & t_{i_{1} j_{2}} \\
t_{i_{2} j_{1}} & t_{i_{2} j_{2}}
\end{array}\right| \geq 0 \text { for } i_{2} \geq i_{1}, j_{2} \geq j_{1}
$$

Assumption 3 Symbol probabilities satisfy $\left(b_{i, m}\right)_{m \in M} \leq_{r}\left(b_{i+1, m}\right)_{m \in M}$ for $i=$ $1, \ldots, X-1$.

Then the Gittins index $\gamma(\pi)$ of each sensor is MLR increasing. Therefore, if the information states of the $N$ sensors are MLR comparable, then the optimal policy $\mu^{*}$ is to pick the sensor with the smallest information state with respect to $M L R$ ordering at each time slot, namely, $a_{k}=\mu^{*}\left(\pi_{k}^{(1)}, \ldots, \pi_{k}^{(N)}\right)=\arg \min \left(\pi_{k}^{(n)}\right), n \in\{1, \ldots, N\}$.

The above theorem says that if the information states of the sensors are MLR comparable, then the optimal policy is a greedy policy. We give the following examples to explain the optimal policies in different scenarios. In a battlefield MANET, assume 
that information leakage is far more important than energy loss. In this case, we can consider two states, \{secure, compromised\}, for each sensor, and all information states are MLR comparable. The optimal policy in this case is to choose the sensor with a higher probability of being in the secure state at each time slot. Similarly, in a civilian MANET, where energy loss is more important than information leakage, we can consider two states, $\{$ high-energy, low-energy\}, for each sensor, and all information states are MLR comparable. The optimal policy in this case is to choose the sensor with a higher probability of being the high-energy state at each time slot. In Simulation Results and Discussions, we have some simulation results in Figs. 6 and 9 about battlefield MANETs and civilian MANETs.

In the following, we show that, under reasonable conditions on the matrices of each node in MANETs, the distributed continuous user authentication and intrusion detection system meets the above three assumptions. Assumption 1 shows that for an arbitrary sensor, the cost in state $i$ is less than or equal to that in state $i+1$. In the distributed continuous user authentication and intrusion detection scheduling problem, the cost vector of each sensor always meets this assumption, since there is more information leakage when a chosen node is in a more dangerous state than when it is in a safer state. Therefore, the cost of selecting a node in the more secure and higher energy state is lower than that of selecting a more compromised and lower energy node.

Assumption 2 holds in the following situation. Due to continuity arguments, if the state of a sensor is $x_{i}, 1 \leq i \leq X$ at time $k$, then at time $k+1$, it is reasonable to assume that it is either still in state $x_{i}$, or, with a lower probability, in the neighboring states $x_{i+1}$ or $x_{i-1}$. Therefore, in our proposed scheme, each sensor can be modeled as a $X$-state Markov chain with diagonally dominant tridiagonal transition probability matrix $T$, where $t_{i j}=0$ for $j \geq i+2$ and $j \leq i-2$. The following matrix is an 
example of a diagonally dominant tridiagonal matrix.

$$
\left(\begin{array}{cccc}
0.9 & 0.1 & 0 & 0 \\
0.1 & 0.8 & 0.1 & 0 \\
0 & 0.1 & 0.8 & 0.1 \\
0 & 0 & 0.1 & 0.9
\end{array}\right) .
$$

According to [123], a necessary and sufficient condition for tridiagonal matrix $T$ to meet Assumption 2 is that $t_{i, i} t_{i+1, i+1} \geq t_{i, i+1} t_{i+1, i}$.

Several common observation probability models for MANETs that satisfy Assumption 3 are listed as follows:

1. Each sensor measures the target in quantized Gaussian noise.

2. Observation probabilities die geometrically fast with the error between the reported observation $y$ and the real state $x$.

3. The value the sensor reports is never more than one discrete value away from the true value. Therefore, $B$ matrix is the following $X \times X$ tridiagonal matrix:

$$
\left(\begin{array}{ccccc}
p_{1} & 1-p_{1} & 0 & 0 & \ldots \\
\frac{1-p_{2}}{2} & p_{2} & \frac{1-p_{2}}{2} & 0 & \ldots \\
0 & \frac{1-p_{3}}{2} & p_{3} & \frac{1-p_{3}}{2} & \ldots \\
0 & 0 & 1-p_{4} & p_{4} & \ldots \\
\vdots & \vdots & \vdots & \vdots & \ddots
\end{array}\right)
$$


In the following, we assume that, in our proposed scheme, vector $C$, matrix $T$, and matrix $B$ of each sensor meet all of the three assumptions in Theorem 1. The Gittins index $\gamma(\pi)$ is monotone increasing. Therefore, if the information states of the $N$ sensors are MLR comparable, the optimal policy is to pick the authentication or intrusion detection sensor with the smallest information state with respect to MLR ordering.

\subsubsection{Approximations to the Gittins Index}

When there are only 2 states for the $N$ sensors, namely $\{($ secure, high_energy), (compromised, low_energy)\}, their information states are always MLR comparable, and the optimal policy will be the same as above. For more states than 2, when the information states are MLR comparable, the optimal policy can be used directly. However, since for $\geq 3$, MLR is a partial ordering, the $N$ information states $\pi^{(1)}, \ldots, \pi^{(N)}$ are not necessarily MLR comparable at each time instant. When the trajectories of the information states are not MLR comparable, the following approximations can be used, by projecting the non-MLR comparable information state onto the nearest MLR comparable state.

In the proposed scheme, each sensor starts from the (secure, high_energy) state, which means that the information state equal to $(1,0, \ldots, 0)$, where all elements equal to zero, except the first. Therefore, the information states of all $N$ sensors are identical. Assume at time instant $k$, the information states of all $N$ sensors are MLR comparable. Let $\sigma(1), \ldots, \sigma(N)$ denote the permutation of $(1, \ldots, N)$, so that $\pi_{k}^{\sigma(1)} \leq_{r} \pi_{k}^{\sigma(2)} \leq_{r} \cdots \leq_{r} \pi_{k}^{\sigma(N)}$. From the above theorem, the optimal action is $a_{k}=\sigma(1)$. But at the time $k+1$, the updated information state $\pi_{k+1}^{\sigma(1)}$ may not be MLR comparable with the other $N-1$ information states. When the updated information state is not MLR comparable with the other information states, it can be 
projected to the nearest information state, denoted $\bar{\pi}$, in the simplex $\Psi$ that is MLR comparable with the other $N-1$ information states. That is, at time $k+1$, we solve the following $N$ optimization problems:

$$
\begin{aligned}
G\left(\bar{\pi}^{(1)}\right)= & \min _{\bar{\pi} \in \Psi}\left\|\bar{\pi}-\pi_{k+1}^{\sigma(1)}\right\| \text { subject to } \bar{\pi} \leq_{r} \pi_{k}^{\sigma(2)} \\
G\left(\bar{\pi}^{(n)}\right)= & \min _{\bar{\pi} \in \Psi}\left\|\bar{\pi}-\pi_{k+1}^{\sigma(1)}\right\| \text { subject to } \\
& \pi_{k}^{\sigma(n)} \leq_{r} \bar{\pi} \leq_{r} \pi_{k}^{\sigma(n+1)}, n=2, \ldots, N-1 \\
G\left(\bar{\pi}^{(N)}\right)= & \min _{\bar{\pi} \in \Psi}\left\|\bar{\pi}-\pi_{k+1}^{\sigma(1)}\right\| \text { subject to } \pi_{k}^{\sigma(N)} \leq_{r} \bar{\pi} .
\end{aligned}
$$

Here, $\|$.$\| denotes some norm, and the 2-norm is used in our simulation. In the equa-$ tions, $G$ and $\bar{\pi}_{n}$ denote the minimum value and minimum solution for each equation, respectively. Finally, set $\pi_{k+1}^{\sigma(1)}=\arg \min _{\bar{\pi}_{n}} G\left(\bar{\pi}_{n}\right)$. The above $N$ problems are straightforwardly shown to be convex optimization problems and can be solved efficiently in real time. Thus, all the information states at time $k+1$ are now MLR comparable, and the optimal action $a_{k+1}$ is choosing the node with the smallest information state with respect to MLR ordering.

\subsubsection{Computational Complexity and Communication Over- head}

In the centralized scheme [35], the whole network is formulated as a single POMDP, and a centralized controller is needed to schedule authentication and intrusion detection in the whole network. Since the state space of the POMDP in the centralized scheme in [35] grows exponentially with the number of scheduled sensors, solving the POMDP can be computationally intractable [8]. By contrast, in the proposed scheme, the optimal policy can be found by a Gittins index rule, which means that 
the scheduling problem only needs to solve the individual POMDPs for each sensor. Therefore, the computational complexity of the proposed distributed scheme is dramatically decreased. The computational complexity can be further reduced in the structural results. The corresponding simulation results about computational complexity will be presented in Subsection 3.3.4.1.

There are some trade-offs within the proposed scheme. For example, in the realtime sensor selection process, each sensor can broadcast the new Gittins index to the other sensors only in certain time slots in order to further decrease the communication overhead. However, the cost becomes higher since the other sensors have to make decision based on out-of-date Gittins indices. In the extreme case, if sensors do not broadcast their Gittins indices to the other sensors at all, sensors have to be chosen randomly at each time slot. The results in Fig. 3.13 show that the average cost of this random scheme is higher than that in the proposed scheme.

\subsubsection{Simulation Results and Discussions}

In this section, we use computer simulations to compare the performance of the centralized scheme and the proposed distributed scheme, and the performance of the two methods (the value iteration algorithm and the structural results method) used in the proposed scheme. Examples of the state transition probability matrices of the 
iris sensor, fingerprint sensor and IDS, when they are active, are defined as follows:

$$
\begin{aligned}
T^{(1)}= & ((0.912,0.088,0.0,0.0),(0.025,0.950,0.025,0.0), \\
& (0.0,0.044,0.912,0.044),(0.0,0.0,0.05,0.95)), \\
T^{(2)}= & ((0.784,0.216,0.0,0.0),(0.1,0.8,0.1,0.0), \\
& (0.0,0.059,0.882,0.059),(0.0,0.0,0.1,0.9)), \\
T^{(3)}= & ((0.9702,0.0298,0.0,0.0),(0.01,0.98,0.01,0.0), \\
& (0.0,0.0149,0.9702,0.0149),(0.0,0.0,0.02,0.98)) .
\end{aligned}
$$

The observation probability matrix of each node is identical to its state transition probability matrix. The cost vectors are defined as: $C^{(1)}=(3,8,20,40), C^{(2)}=$ $(2,7,22,45), C^{(3)}=(1,4,25,50)$. These specific values are used in all simulations. In the simulations presented in Table 4.1, these matricies were modified to create a variety of similar node types. Since there is more potential for information leakage when a node is in the compromised state than in the secure state, the information leakage cost of selecting a secure node is lower than that of selecting a compromised node $^{3}$. The above matrices vary in different applications. For example, the cost value of a sensor in a more compromised state needs to be set much higher than that in a more secure state especially in a battlefield network, so a more secure sensor can be chosen at each time slot. However, in a civilian network, especially for a energyconcerned network, the cost value of a sensor in a lower energy state needs to be set much higher than that in a higher energy state, so a higher energy sensor can be chosen at each time slot.

\footnotetext{
${ }^{3}$ For example, a compromised node could intentionally introduce collisions in a cryptographic protocol.
} 


\subsubsection{Computational Efficiency Comparison}

Simulations are performed to compare the computational efficiency in the centralized scheme [35] and the proposed distributed scheme. Table 3.1 shows the computation time spent in the centralized scheme and the proposed distributed scheme in the offline and on-line parts, as the total number of node types in the MANET varies from 2 to 50. For the value iteration algorithm, the on-line computation time is of the same level as that of the structural results method. The table also shows that the off-line time is the dominant part for the value iteration algorithm. The computation time dramatically increase when the number of node types changes from 2 to 4 : from 0.03 seconds to more than 8 hours. In the structural results method, a quicksort algorithm with MLR ordering is used to sort the sensors by current information states. Each value is the averaged result of 1000 simulations. The off-line computation time for the structural results method is always equal to 0 , since the method is only used for on-line sensor scheduling. The computation time of the structural results method slightly increase with the increasing type of the nodes in the network. This shows that the structural results are practically useful in real MANETs.

\subsubsection{Policies Derived from the Structural Results}

Fig. 3.12 shows an example of the policy for optimal scheduling in the proposed scheme using the structural results method. In this example, there are three sensors, one iris, one fingerprint, and one IDS. From Fig. 3.12, we can see that authentication and IDS are scheduled dynamically as the simulation runs to minimize the information leakage and maximize the network lifetime in the MANET.

We also investigate how the initial states of the sensors affect the MLR noncomparable percentage between the chosen sensor and the idle sensors using the structural results method, which affects whether or not the structural results method 
Table 3.1: The computation time in the centralized scheme and the proposed distributed scheme.

\begin{tabular}{|c|c|c|c|c|c|}
\hline Scheme & Method & $\begin{array}{l}2 \text { node } \\
\text { types }\end{array}$ & $\begin{array}{l}4 \text { node } \\
\text { types }\end{array}$ & $\begin{array}{l}20 \text { node } \\
\text { types }\end{array}$ & $\begin{array}{l}50 \text { node } \\
\text { types }\end{array}$ \\
\hline $\begin{array}{l}\text { Centralized } \\
\text { scheme }\end{array}$ & $\begin{array}{l}\text { Incremental prun- } \\
\text { ing algorithm }\end{array}$ & $2 \mathrm{~h} 32 \mathrm{~m} 10 \mathrm{~s}$ & unfeasible & unfeasible & unfeasible \\
\hline \multirow{4}{*}{$\begin{array}{l}\text { Proposed } \\
\text { distributed } \\
\text { scheme }\end{array}$} & $\begin{array}{l}\text { Structural results } \\
\text { (off-line) }\end{array}$ & 0 & 0 & 0 & 0 \\
\hline & $\begin{array}{l}\text { Structural results } \\
\text { (on-line) }\end{array}$ & $0.0427 \mathrm{~s}$ & $0.0576 \mathrm{~s}$ & $0.2337 \mathrm{~s}$ & $0.5960 \mathrm{~s}$ \\
\hline & $\begin{array}{l}\text { Value iteration al- } \\
\text { gorithm (off-line) }\end{array}$ & $0.03 \mathrm{~s}$ & $8 \mathrm{~h} 1 \mathrm{~m} 22 \mathrm{~s}$ & unfeasible & unfeasible \\
\hline & $\begin{array}{l}\text { Value iteration al- } \\
\text { gorithm (on-line) }\end{array}$ & $0.0379 \mathrm{~s}$ & $0.0531 \mathrm{~s}$ & - & - \\
\hline
\end{tabular}
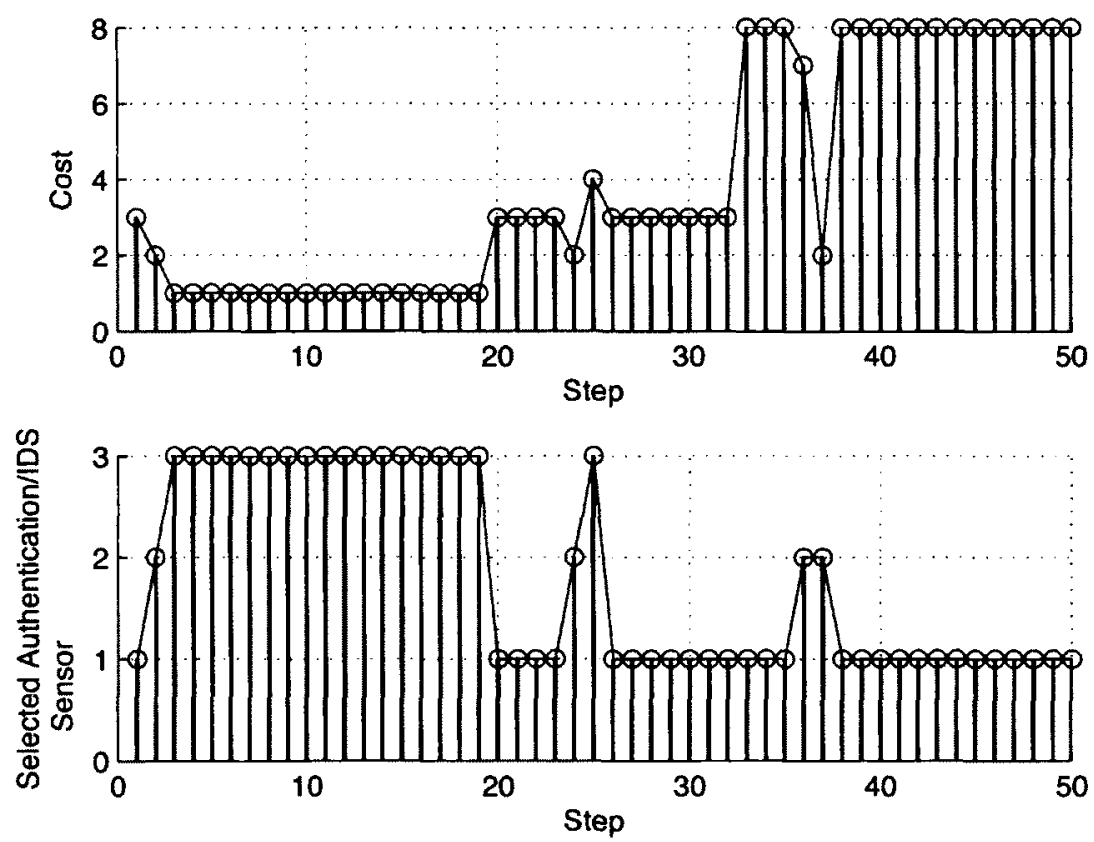

Figure 3.12: An example policy derived from the structural results method (1: Iris; 2: Fingerprint; 3: IDS). 
Table 3.2: Percentage of states not MLR comparable in the first 100 steps.

\begin{tabular}{|l|c|c|c|c|c|}
\hline$\pi_{0}^{(n)}$ & $e_{1}$ & $e_{2}$ & $e_{3}$ & $e_{4}$ & $\pi_{0}^{(n)}(i)=1 / 4$ \\
\hline $\begin{array}{l}\text { Percentage of states not } \\
\text { MLR comparable }\end{array}$ & 36.36 & 99.50 & 49.01 & $1.4000 \mathrm{e}-004$ & 63.25 \\
\hline
\end{tabular}

can be directly used in that time slot. Each value is the averaged result of 1000 simulations. Table 3.2 shows the percentage of sensor choices in the first 100 steps where the updated information state of the chosen sensor is not MLR comparable, for different choices of initial sensor state. The table shows that the percentage of choices leading to non-MLR-comparable states is near to 0 when the sensors start in the fourth state, since the sensors have a high probability of remaining in the fourth state.

\subsubsection{Performance Comparison}

We perform simulations to compare the performance of the centralized scheme, the random choice scheme and the proposed distributed scheme, and the performance of the two methods used in the proposed scheme. Fig. 3.13 illustrates the average cost for the first 100 steps of the simulation. Each cost value is the averaged result of 1000 simulations. The figure shows that the average cost of this random scheme is the highest among the three schemes. The figure also shows that the average cost from the proposed distributed scheme is higher than that from the centralized scheme, since the nodes in the centralized scheme can make better decisions as they have complete information. Since the nodes all start from (secure, high-energy), the average costs using of different schemes all begin at a low level, and diverge as over time as the network changes states. The results show that the average cost from the value iteration algorithm and that from the structural results method are very 
close to each other. Fig. 3.14 illustrates the relative information leakage, which is defined as the information leakage of the selected node divided by the information leakage when the node is in the worst state. The same observation is true with the information leakage in Fig. 3.14. This shows the effectiveness of these two methods, both of which can provide optimal policies that minimize the information leakage and maximize the network lifetime. The reasons why the result from the value iteration algorithm is not exactly the same as that from the structural results method are as follows:

1. For the value iteration algorithm, the finite-horizon Gittins index approximation can be made arbitrarily accurate by choosing a sufficiently large horizon $H$, which is a trade-off between performance and computation time.

2. For the structural results method, the updated information state of the chosen sensor has to project to the nearest MLR comparable information state when the updated information state is not MLR comparable to those of the other sensors, therefore some errors are generated. Nevertheless, the errors are small, which make the results from the structural results method very close to those from the value iteration algorithm.

Figs. 3.13 and 3.14 also show that the results from the two methods are quite different in the early steps. The reason is that the sensors all start in the first state, which means their information states are $(1,0,0,0)$. Therefore, they are identical, and one of the sensors is chosen randomly in the structural results method. However, for the value iteration algorithm, the sensor that can minimize the total expected discounted cost is chosen at each time slot. After about 10 steps, the results are very close in these two methods. 


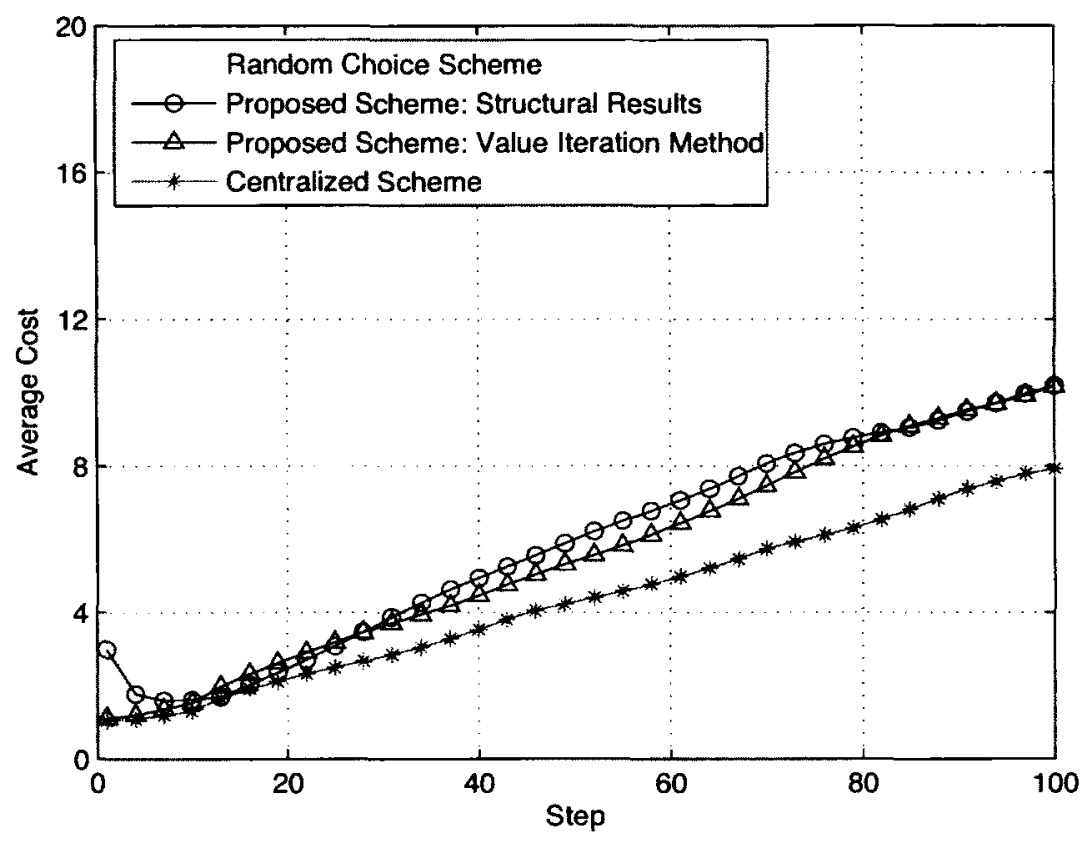

Figure 3.13: Average cost comparison of the centralized scheme, the random choice scheme, and the proposed distributed scheme.

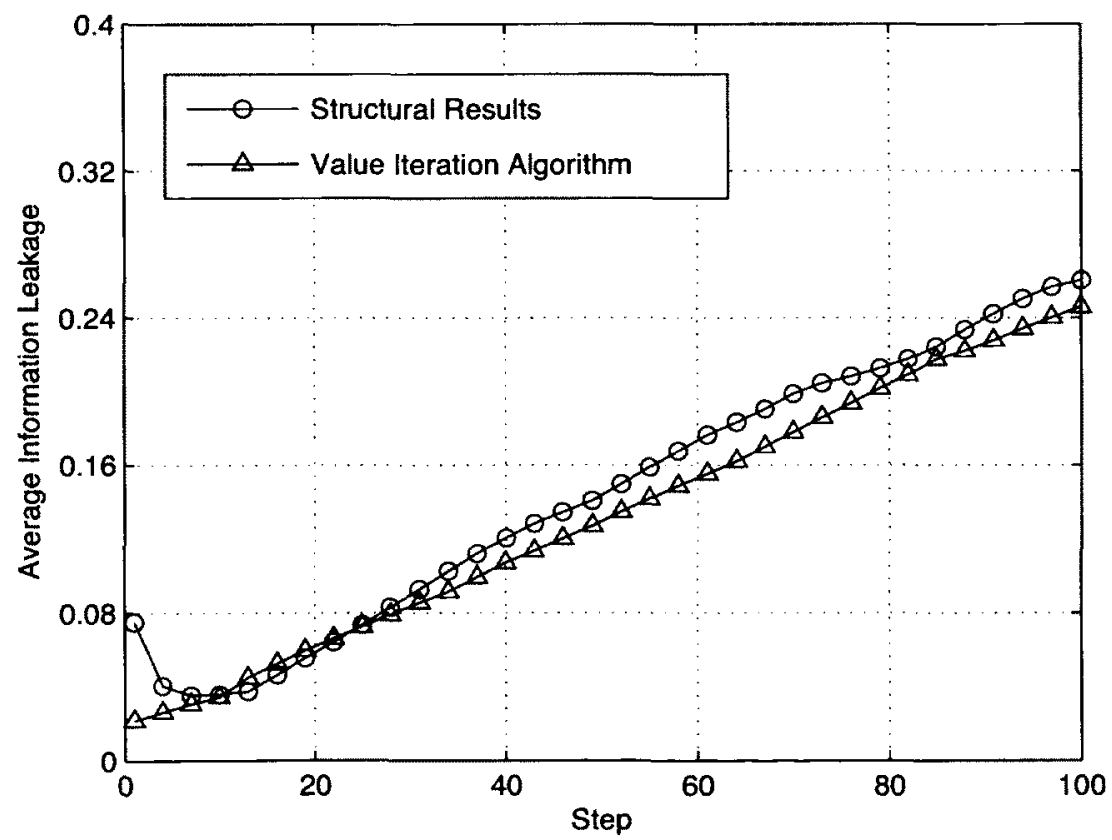

Figure 3.14: Average information leakage comparison between the two methods. 
Different numbers of nodes are also used in the simulations to verify the scalability of the proposed scheme using each method in the battlefield network and civilian network. Fig. 3.15 and Fig. 3.16 show the average cost and the average information leakage within the first 100 steps of the simulation of networks of different sizes. In these simulations, we use the same three types of nodes mentioned earlier. The figures show that the average information leakage in the battlefield network is much smaller than that in the civilian network, since the more secure sensors in the battlefield network are the optimal choices rather than the higher-energy ones in the civilian network. The figures show that the average cost and the average information leakage results for the value iteration algorithm and the structural results method in the battlefield network are similar. The results also show that the average cost and the average information leakage decrease when the number of available nodes in the network increases from 3 to 18 . The reason is that there are more nodes that can be selected for authentication and intrusion detection, so compromised and low-energy nodes can be avoided.

Various state transition probabilities are also used in the simulations to evaluate the dynamic stability of the proposed scheme using each method. Fig. 3.17 shows the average cost using these two methods as the first component in the state transition probability matrix varies from 0.7 to 1.0 , where high state transition probability means that the system is more secure. The results show that the average costs using these two methods are similar. From Fig. 3.17, we can observe that the cost decreases when the system becomes more secure. This is because the information leakage is smaller when the system becomes more secure, therefore the cost is smaller. When the state transition probability reaches 1 , the average cost of the two methods is quite different. The reason is that when the state transition probability reaches one, the information states do not change, and therefore a sensor is randomly chosen at 


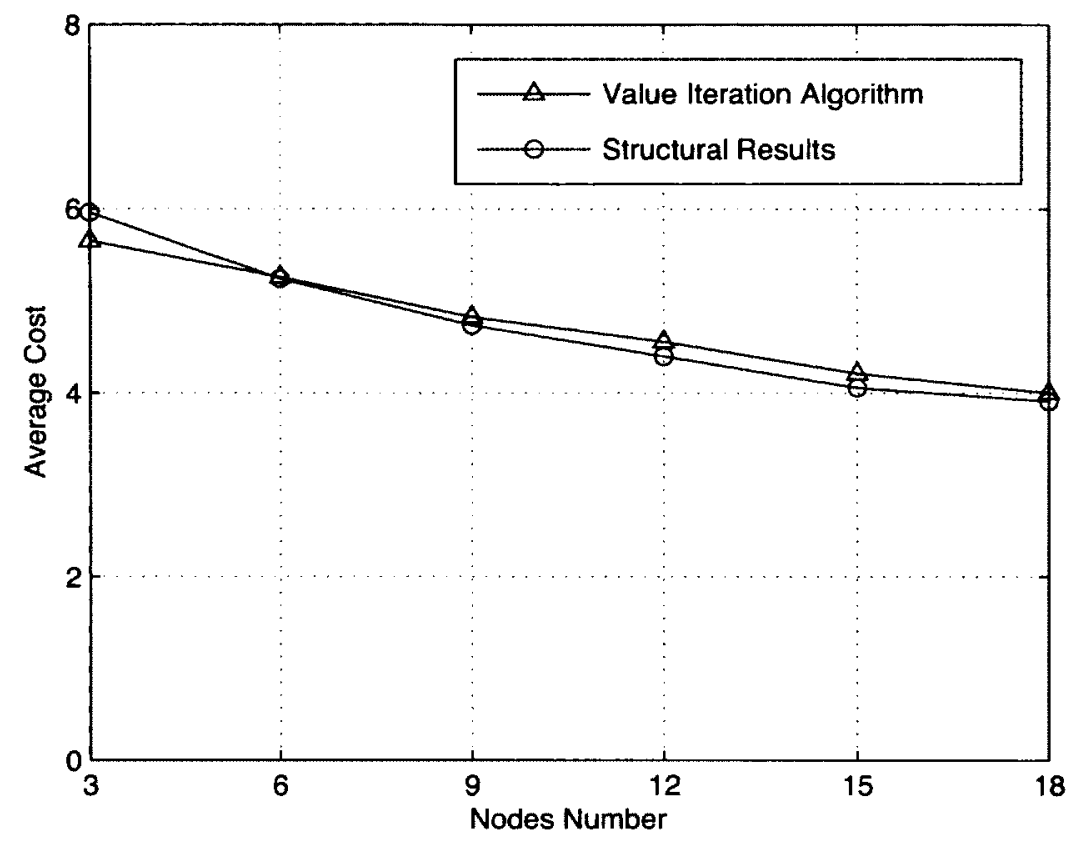

Figure 3.15: Average cost comparison between the two methods with different numbers of nodes.

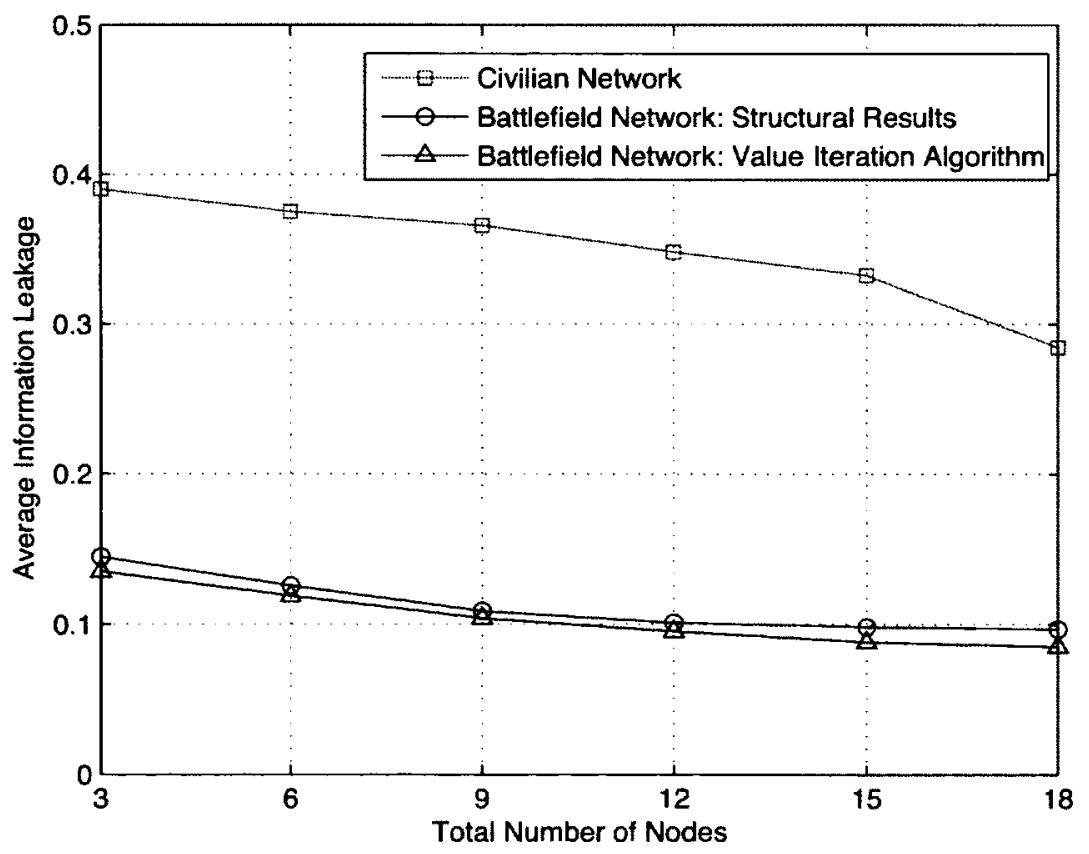

Figure 3.16: Average information leakage comparison between civilian network and battlefield network with different numbers of nodes. 


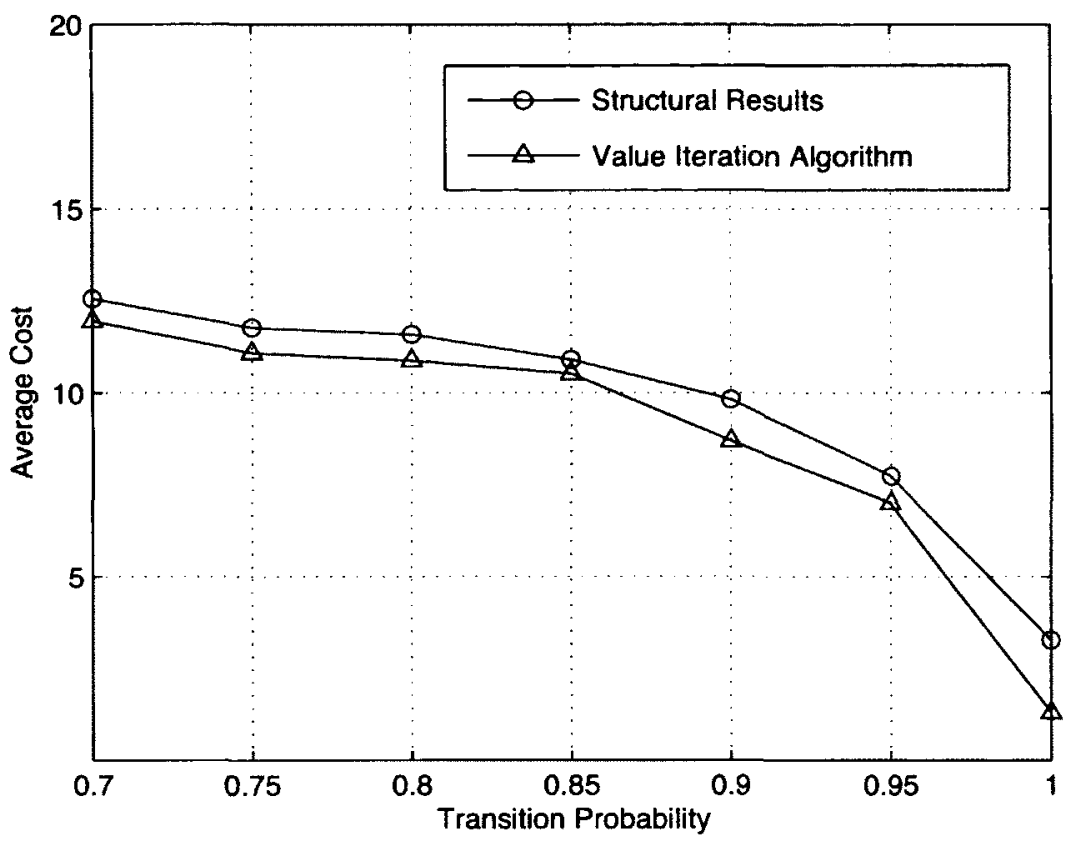

Figure 3.17: Average cost comparison between the two methods with different state transition probabilities.

each time slot. Fig. 3.18 shows the average information leakage within the first 100 steps when the first component in the state transition probability matrix of the IDS varies from 0.7 to 1.0 and all other probability values remain constant. The average information leakage using these two methods are still close. The results show that information leakage remains stable for the first four probabilities, and decreases when the system becomes more secure. The reason for this is that the proposed schemes avoid choosing the compromised nodes. When the state transition probability further increases, the average information leakage decreases.

\subsubsection{Network Lifetime Comparison}

Network lifetime performance has been evaluated for the proposed scheme using each method, which is illustrated in Fig. 3.19. The results show that the network lifetime increases with the total number of nodes. The average network lifetime is much higher 


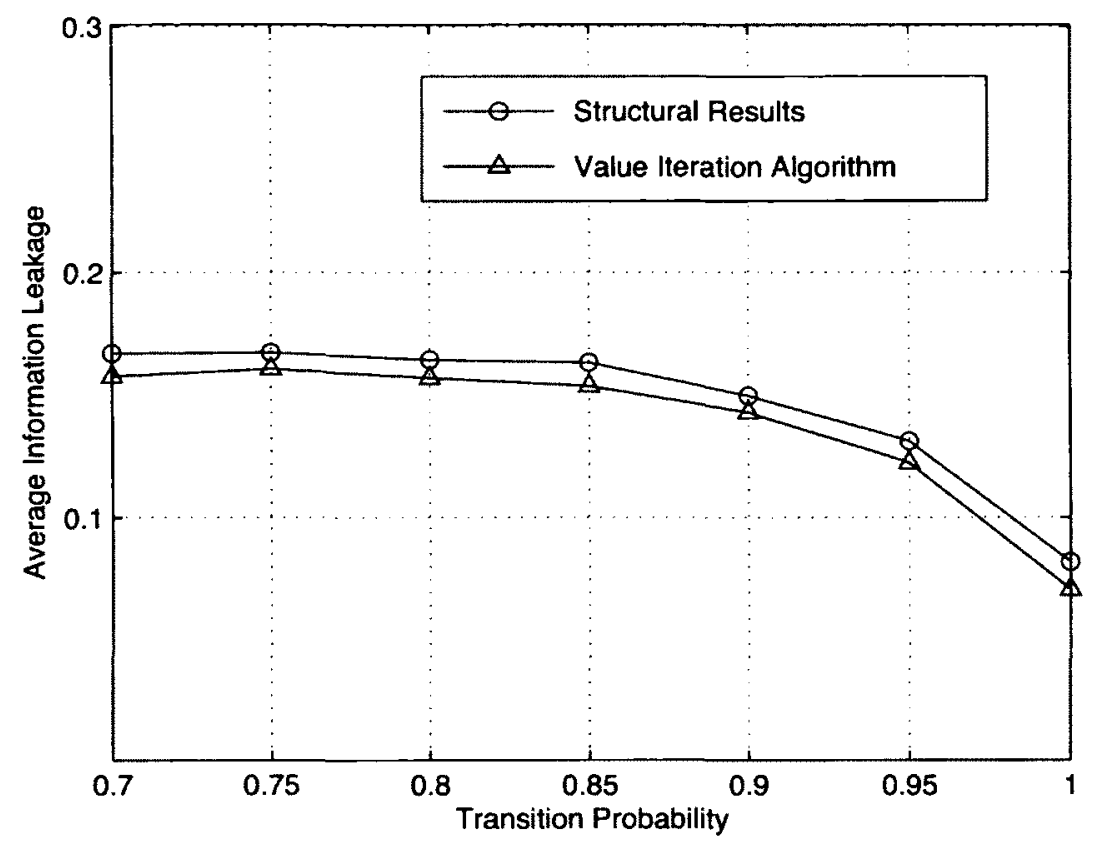

Figure 3.18: Average information leakage comparison between the two methods with different state transition probabilities.

in the civilian network compared to that of the battlefield network, since the higherenergy sensor is chosen at each time slot, with the trade-off being greater information leakage. In the battlefield network, the results using the value iteration algorithm and the structural results method are very close.

\subsection{Conclusion}

Combining continuous authentication and intrusion detection can be an effective approach to improve the security performance in high security green MANETs. We presented a distributed scheme combining authentication and intrusion detection. In the proposed scheme, the most suitable biosensors for authentication or IDSs are dynamically selected based on the current security posture and energy states in different applications. To improve upon this concept, Dempster-Shafer theory was used 


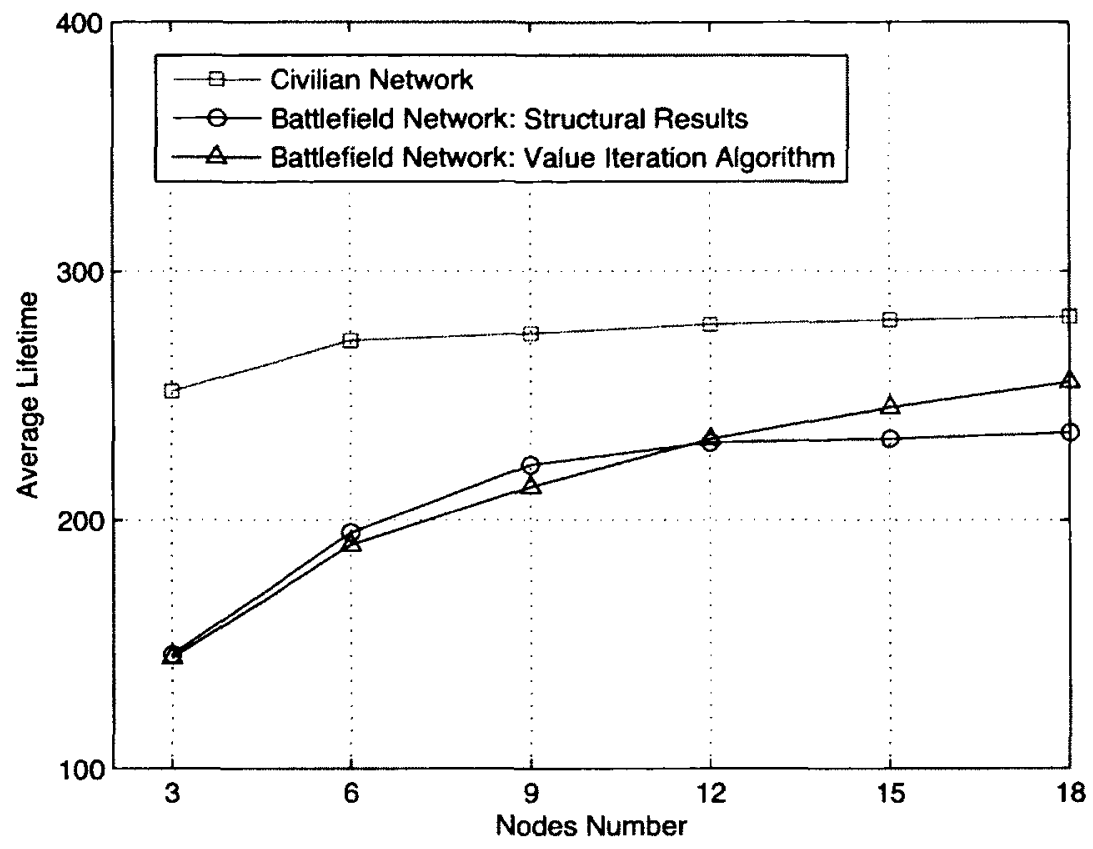

Figure 3.19: Network average lifetime comparison between civilian network and battlefield network with different numbers of network nodes.

for IDS and sensor fusion, when more than one device is used at each time slot. The problem was formulated as a stochastic multi-armed bandit problem, and its optimal policy can be chosen using Gittins indices. We presented a value-iteration algorithm and a structural results method for calculating the Gittins indices of the sensors. Simulation results were presented to show that the proposed scheme can improve network security and increase network lifetime. The system performance of the structural results method is very similar to that of the value iteration algorithm, but with much lower computational complexity. 


\section{Chapter 4}

\section{Stochastic Scheduling and Decision-Making Schemes in Smart Grids}

\subsection{Introduction}

In this chapter, two important issues in smart grids are considered: the unit commitment (UC) scheduling problem in Section 4.2, and the decision-making problem for electricity retailers in Section 4.3. UC scheduling of power generation systems is an important issue in smart grids to effectively coordinate energy demand and generation in order to minimize cost and greenhouse gas emissions, and to avoid blackouts in the event that the natural phenomena necessary to generate renewable energy do not occur [36]. In a retail electricity market, retailers need to procure electricity from various sources with different characteristics and then sell it to customers. The electricity demand of the customers might vary with time. Therefore, retailers need to make effective decisions about electricity sources, the electricity amount they procure and the price to offer to the customers.

The highlights of the research contribution in Section 4.2 are as follows:

- Hidden Markov models are used for modeling the intermittent renewable energy resources. Markov models are an effective approach to characterize the 
correlation structure of the renewable energy outputs [9-11]. Meteorological instability and complex system dynamics (e.g., wind turbine or solar cells malfunction) make it difficult to fully observe the renewable energy system state, so hidden Markov models are appropriate. A Markov-modulated Poisson process (MMPP) model is used to predict the stochastic power demand loads.

- The unit commitment scheduling problem is formulated as a partially observable Markov decision process (POMDP) multi-armed bandit system $[8,114]$, which is a powerful stochastic control modeling framework and has been successfully used in distributed project selection $[114,124]$ and sensor scheduling [123], among others. Based on this formulation, a fully distributed and scalable unit commitment scheduling scheme can be derived.

- A value iteration method is presented to solve the problem. This method works well for a smart grid with a small number of power generation units. However, for a large grid with a variety of units, the value iteration method can become computationally intractable. Therefore, a structural results method is also presented for this problem. The structural results method is easy to implement, which makes the solution practical for the future smart grid with intermittent renewable energy resources and stochastic power demands.

- Simulation results are presented to show the effectiveness of the proposed scheme. It is shown the proposed scheme can significantly reduce the cost and greenhouse gas emissions compared to the schemes that do not consider hidden Markov models for intermittent renewable energy resources and stochastic power demands.

The highlights of the research contribution in Section 4.3 are as follows: 
- We use various utility functions based on concepts from microeconomics to model the electricity customers' preferences and electricity consumption patterns.

- A real-time demand response scheme is used by the customers to adjust their electricity demand to maximize their individual utility.

- We model and analyze the interactions between the retailer and electricity customers as a four-stage Stackelberg game [125]. The first three stages of the game analyze how the retailers should make decisions on which electricity sources to procure electricity from, how much electricity to procure, and what would be the optimal retail price to offer to the customers, in order to maximize profit. The fourth stage of the game shows how customers dynamically adjust their electricity demand with the offered retail price to maximize individual utility.

- Simulation results show the retailer and customers can achieve a higher profit and higher utility using our proposed decision-making scheme and how the system parameters affect the procurement and price decisions.

The work presented in this chapter has resulted in two journal papers $[126,127]$ (under revision) and four conference papers [128-131]. Possible future work in this area is provided in Chapter 6 .

\subsection{Distributed Unit Commitment Scheduling}

In this section, a distributed stochastic UC scheduling scheme is proposed in smart grids with intermittent renewable energy resources and stochastic power demand loads. This section is organized as follows. Section 4.2.1 introduces the system models. Section 4.2.2 describes the formulation and solutions to the scheduling problem. 
Section 4.2 .3 presents the simulation results.

\subsubsection{System Models}

In the future, solar panels, wind turbines and traditional fossil fuel power generators are integrated into smart grids. Wind turbines and solar panels might be located far from demand points or each other in order to improve the reliability and to lower variability of the system [132]. Since both power resources highly depend on weather conditions, it is possible that power fluctuations occur. Therefore, fossil fuel power generators are also used in order to mitigate or even cancel out the fluctuations. Realtime pricing DSM is used in smart grids, since it is an effective tool to influence the electricity consumption behavior of customers and also reflects variations in the cost of the energy supply. Two kinds of energy users exist in smart grids: traditional energy users, and opportunistic energy users, who introduce uncertainties on the demand side.

The relationships between supply side management and unit commitment, as well as demand-side management and unit commitment are illustrated in Fig. 4.1. Both the status of the generators and the status of the demand side determine which units will be committed. In turn, unit commitment affects demand, which produces a feedback loop, and is different from the traditional one-way effect that the demand side has on unit commitment. The power generation units need to be integrated together using smart grid communications in order to serve the stochastic demand loads while satisfying economic, reliability and environmental criteria. Therefore, it is critical to optimally schedule these units, considering stochastic demand loads, costs, reliability and pollutant emissions. 


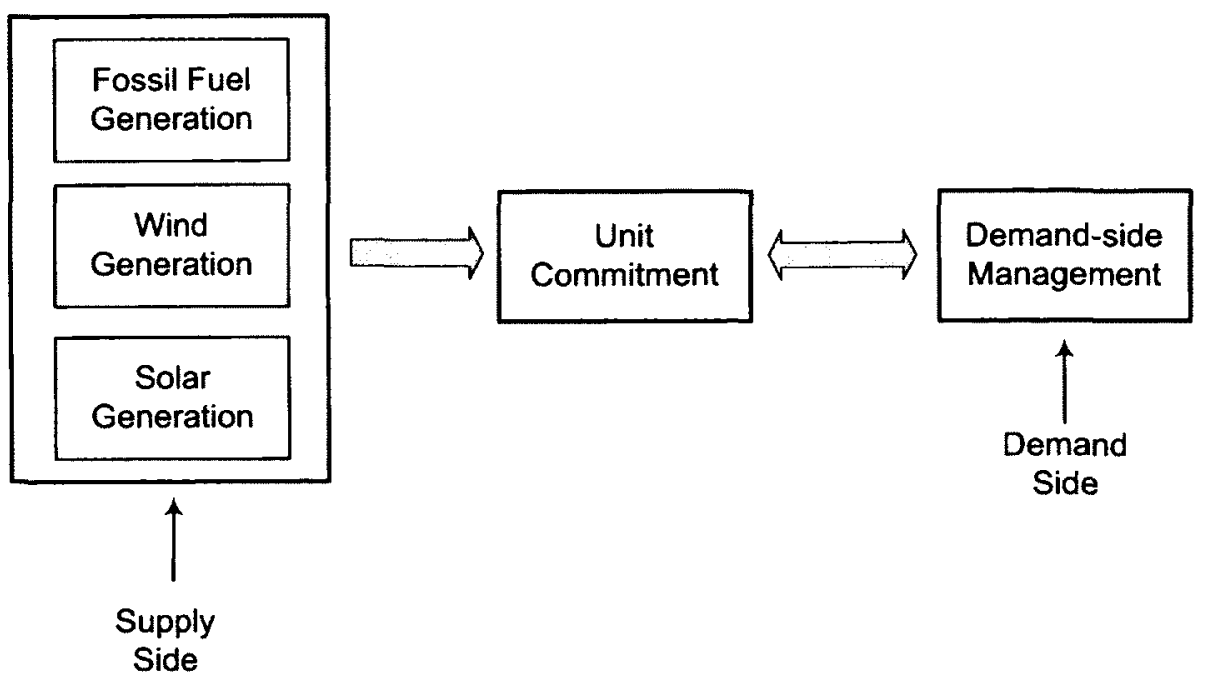

Figure 4.1: The relationships between supply side management, demand-side management and unit commitment.

\subsubsection{Renewable Energy Model}

In this section, wind power and solar power mean the electric power generated by wind turbines or solar panels rather than the input wind power or solar radiation power. Wind turbine and solar panel may mean an individual energy generation unit or a cluster of such units as a farm, depending on the context under consideration. 


\section{Wind Power Model}

The energy produced by a wind turbine per unit time when it is available is as follows [133]:

$$
P_{w}=\left\{\begin{aligned}
0, & v<v_{\text {cutin }} \\
F v^{3}, & v_{\text {cutin }} \leq v \leq \sqrt[3]{P_{\max } / F} \\
P_{\max }, & \sqrt[3]{P_{\max } / F}<v<v_{\text {cutout }} \\
0, & v_{\text {cutout }} \leq v
\end{aligned}\right.
$$

In the above equation, $v_{\text {cutin }}$ and $v_{\text {cutout }}$ are wind speeds at which the turbine starts to generate power, and the turbine must be turned away from the wind to protect the blades, respectively. $P_{\max }$ represents the maximal power the wind turbine can produce. $F=1 / 2 \zeta \rho \pi R^{2}$, where $\zeta$ is the efficiency of the turbine. $\rho$ represents the air density which is roughly $1.23 \mathrm{~kg}$ per cubic meter at sea level [134].

\section{Solar Power Model}

For each photovoltaic solar cell, the amount of generated current $I_{c}$ depends on the intensity of solar radiation $\theta$. Given by a diode model, the relationship between its voltage $V_{c}$ and current $I_{c}$ can be described as follows [135]:

$$
I_{c}(\theta)=\left(D_{0}+D_{1} T_{c}\right) \theta-I_{0}(\cdot)-\frac{\left(V_{c}-I_{c} R_{s}\right)}{R_{s h}}
$$

where $D_{0}$ and $D_{1}$ are constant coefficients, $T_{c}$ is the temperature of the solar cell, $I_{0}(\cdot)$ is a function of diode saturated current, and $R_{s}$ and $R_{s h}$ are series and shunt resistances, respectively. 


\subsubsection{Power Generation Unit State Model}

Assume that there are $G_{w}$ wind turbines, $G_{s}$ solar panels and $G_{f}$ fossil fuel power generation units in smart grids, for $N$ units total. Assume that the time axis is divided into equal time slots, which correspond to the time intervals between two scheduling decisions.

Let the state of a generator $n, n \in\{1,2, \ldots, N\}$, be $s_{k}^{(n)}$ at time slot $k$. Each state represents the power supply level of generator $n$. The power supply of each generator can be divided into $L$ discrete levels. Therefore, the power supply state space $\mathbb{S}$ includes all the power supply states $\left\{s_{1}, \ldots, s_{L}\right\}$. The state of generator $n$ evolves according to a $L$-state first order Markov chain with state transition probability matrix $J^{(n)}$, which is described as follows:

$$
J^{(n)}=\left(s_{i j}^{(n)}\right)_{i, j \in \mathbb{S}}, \text { where } s_{i j}^{(n)}=P\left(s_{k+1}^{(n)}=j \mid s_{k}^{(n)}=i\right)
$$

Since meteorological instability and complex system dynamics (e.g., wind turbine or solar cells malfunction) make it difficult to fully observe the system state $[54,55]$, hidden Markov models are used to describe intermittent renewable energy units. The observation of selected generator $n$ 's power level state is denoted as $y_{s k}^{(n)}$ at time slot $k$, which belongs to a finite set $\mathbb{M}_{s n}$, and $\left|\mathbb{M}_{s n}\right|$ denotes the number of possible observations of the generator $n$ 's power generation level state. If generator $n$ is picked at time $k$, and the system state $s_{k}^{(n)}$ equals to $i$, the probability of observation $m$ will be obtained from generator $n$ is denoted as:

$$
b_{i}\left(a_{k}=n, y_{s k}^{(n)}=m\right)=P\left(y_{s k}^{(n)}=m \mid s_{k}^{(n)}=i, a_{k}=n\right)
$$




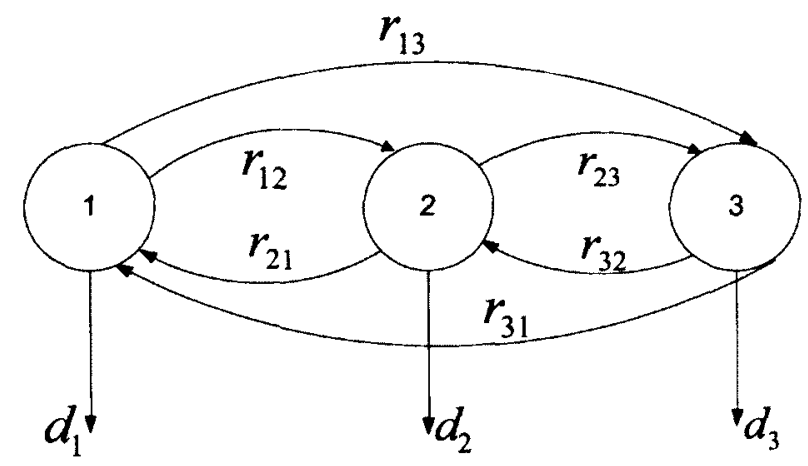

Figure 4.2: An example of 3-state Markov-modulated Poisson process (MMPP).

where $i \in \mathbb{S}$, and $m \in \mathbb{M}_{s n}$. Define the observation matrix of generator $n$ as:

$$
B_{s}^{(n)}\left(y_{s k}^{(n)}=m\right)=\operatorname{diag}\left[b_{1}\left(a_{k}, m\right), \ldots, b_{L}\left(a_{k}, m\right)\right]
$$

\subsubsection{Power Demand Model}

A MMPP is used to model the stochastic power demands. MMPPs have been extensively used for modeling the processes in various applications, such as communications $[136,137]$. MMPP can qualitatively model the time-varying arrival rate and capture some of the important correlations between the inter-arrival times while still remain analytically tractable [136].

The MMPP for modeling power demands consists of a $Q$-state Markov chain. Let the state of the demand arrival rate be $d_{q}, d_{q} \in \mathbb{D}=\left\{D_{1}, D_{2}, \ldots, D_{Q}\right\}$, where $Q$ is the number of demand state levels. The arrival power demand resides in state $d_{q}$ for a mean time of $1 / r_{q}$ with rate $d_{q}$. An example of 3 -state MMPP is presented in Fig. 4.2 .

In the proposed scheme, the MMPP is specified by the infinitesimal generator matrix $G M$ as follows [138]: 


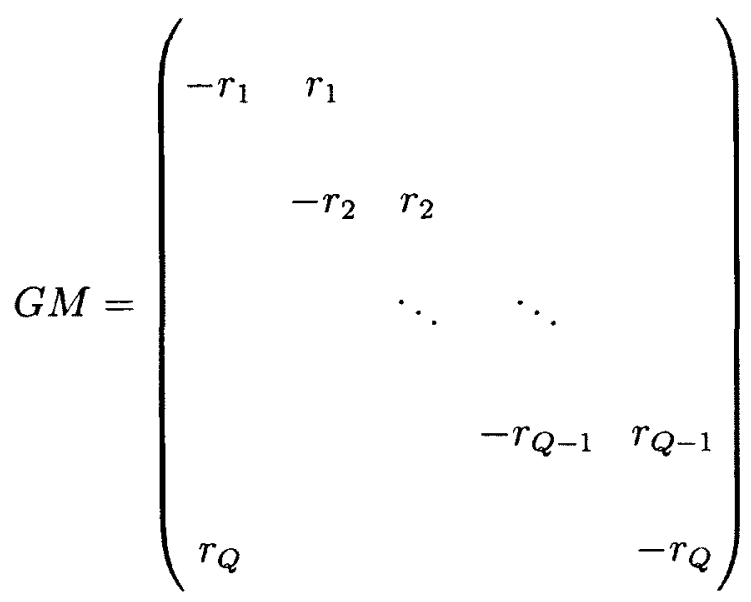

Since matrix GM models a continuous-time Markov chain, uniformization [139] is used to obtain the equivalent discrete-time transition probability matrix $T$ for the stochastic power demand as follows [10]:

$$
T=\frac{G M}{V+I}
$$

where $\vee \geq \min _{q}\left(\left|[G M]_{q, q}\right|\right)$ (i.e., $\vee$ is greater than or equal to the absolute value of the minimum diagonal element in $G M$ ) and $I$ denotes an identity matrix. The observation probability matrix $B_{t}$ of the hidden Markov chain for power demand state can be obtained based on the conditional probability function of the actual demand states and observation states. $B^{(n)}$ denotes the probabilities of the observation $y_{k+1}^{(n)}$ acquired when generator $n$ is picked at time $k+1$ given each state of the Markov chain. Matrix $B^{(n)}$ can be computed based on matrix $B_{s}^{(n)}$ and matrix $B_{t}, B^{(n)}=B_{s}^{(n)} \otimes B_{t}$, where $\otimes$ denotes the Kronecker product. Matrix $U^{(n)}$ can be computed based on matrix $J^{(n)}$ and matrix $T, U^{(n)}=J^{(n)} \otimes T$. 


\subsubsection{Cost Model}

In order to make a good trade-off among electricity cost, customer interruption, and green gas emissions, we define utility cost, customer interruption cost (CIC) and pollutant emission cost (PEC) in the cost function as follows.

At time slot $k$, let unit $n$ be selected, based on the history information $Y_{k}^{(n)}$. The instantaneous cost $C\left(x_{k}^{(n)}, n\right)$ incurred at time $k$ is as follows $[140,141]$ :

$$
C\left(x_{k}^{(n)}, n\right)=w_{U} \underbrace{U C_{k}^{(n)}\left(x_{k}^{(n)}\right)}_{\text {utility }}+w_{C} \underbrace{C I C_{k}^{(n)}\left(x_{k}^{(n)}\right)}_{\text {interruption }}+w_{P} \underbrace{P E C_{k}^{(n)}\left(x_{k}^{(n)}\right)}_{\text {pollution }},
$$

where $w_{U}, w_{C}$ and $w_{P}$ are the weights associated with $U C_{k}^{(n)}, C I C_{k}^{(n)}$ and $P E C_{k}^{(n)}$, respectively. The wight factors indicate the relative importance of each cost, which can be set differently in different scenarios. For example, in most developing countries, pollutant emission are not considered. In this case, $w_{P}$ will be set to $0 . x_{k}^{(n)}=$ $\left(s_{k}^{(n)}, d_{k}\right)$, which includes the state of generation unit $n$ and the demand state at time $k$. The state space of $x_{k}^{(n)}$ is $\mathbb{X}_{n}$.

Utility cost $U C_{k}^{(n)}$ includes the average costs associated with the required investments and operations of the chosen generator $n$ during time slot $k$, when a renewable energy generator is chosen. If a fuel generator is chosen, the utility cost for purchasing power at time $k$ can be calculated as: $U C_{k}^{(n)}\left(s_{k}^{(n)}, d_{k}\right)=p_{k} \times \varphi\left(p_{k}\right)$, where $p_{k}$ is the power purchased at time $k$, and $\varphi\left(p_{k}\right)$ is the power price. Function $\varphi(\cdot)$ is an increasing, differentiable convex function, which reflects that each unit of additional power needed to satisfy increasing demand becomes more expensive to obtain and make available to the consumers. Therefore, $U C_{k}^{(n)}\left(s_{k}^{(n)}, d_{k}\right)$ is an increasing, differentiable convex function.

Customer interruption cost $C I C_{k}^{(n)}$ is directly related to the type of customers 
and the duration of interruptions (i.e. the length of time slot $k$ ), which is defined as follows:

$$
C I C_{k}^{(n)}=\left\{\begin{array}{rc}
F(\Delta), & \Delta>0 \\
0, & \Delta \leq 0
\end{array}\right.
$$

where $\Delta$ equals to the power demand subtracting to the power generated from generator $n$ at time slot $k . F(\Delta)=\sum_{z=1}^{Z} f_{z}\left(\Delta_{z}\right)$, where $\Delta=\Delta_{1}+\cdots+\Delta_{z}$. Function $f_{z}(\cdot)$ is the CIC in customer sector $z$, and $Z$ is the number of customer sectors. Function $f_{z}(\cdot)$ can be obtained through surveying different customer groups [142].

With the increasing concerns about environmental protection, stricter regulations on pollutant emissions have been introduced, often including financial penalties associated with emissions (e.g., carbon credits), as well as non-financial costs associated with environmental damage, which is represented as financial penalties as well in our model. There are no pollutant emissions for renewable energy generation units. The amount power generators must pay depends on fuel consumptions $p_{k}$, which can be calculated as follows [140]:

$$
P E C_{k}^{(n)}=\alpha+\varepsilon \times p_{k}+\gamma \times p_{k}^{2},
$$

where $\alpha, \varepsilon$, and $\gamma$ are the coefficients approximating the generator emission characteristics.

In the proposed scheme, the number of scheduled units at each time slot is determined by the renewable energy generation situation and the total power demand loads. Assume that $G$ generation units are chosen at each time slot. Define a set $S G \subset\{1, \ldots, N\},|S G|=G$. Therefore, the total expected discounted cost over an 
infinite-time horizon is given by:

$$
J_{\mu}=E\left[\sum_{k=0}^{\infty}\left(\beta^{k} \times \sum_{n=1}^{G} C\left(x_{k}^{(n)}, n\right)\right)\right], n \in S G,
$$

where $\beta(0 \leq \beta<1)$ is the discounted factor, which models the fact future cost is worth less than immediate cost, because the future is less certain. The objective is to find the optimal stationary policy $\mu^{*}=\arg \min _{\mu \in \eta} J_{\mu}$ to minimize the cost in (4.9).

\subsubsection{Formulation and Solutions to Stochastic Unit Schedul- ing Problem}

The above scheduling problem is formulated as a POMDP multi-armed bandit problem $[8,114]$, which is a powerful stochastic control modeling framework that has been successfully used in distributed project selection $[114,124]$ and sensor scheduling [123], among other areas. Based on this formulation, a fully distributed and scalable unit commitment scheduling scheme can be derived. In addition, a value iteration method and a structural results method are presented as follows as possible solutions to this problem.

\subsubsection{Value Iteration Method}

A value iteration method can be used to compute the Gittins index. Define two positive real numbers $\bar{M}^{(n)}$ and $M^{(n)}$ for an arbitrary generation unit $n$ as

$$
\bar{M}^{(n)} \triangleq \max _{i \in \mathbb{X}_{n}} \frac{R\left(x_{k}^{(n)}=i, a_{k}=n\right)}{1-\beta}, 0 \leq M^{(n)} \leq \bar{M}^{(n)},
$$

where $R(\cdot)$ is the corresponding reward column vector of cost vector $C(\cdot)$. For simplification, the superscript $n$ in $\bar{M}^{(n)}$ and $M^{(n)}$ is omitted in the following calculation. 
The Gittins index of unit $n$ can be defined as:

$$
\gamma^{(n)}\left(\pi^{(n)}\right) \triangleq \min \left\{M: V^{(n)}\left(\pi^{(n)}, M\right)=M\right\}
$$

where $M$ denotes the parameterized retirement reward, and $V^{(n)}\left(\pi^{(n)}, M\right)$ satisfies the functional Bellman's recursion. The $H$-th order approximation of $V^{(n)}\left(\pi^{(n)}, M\right)$ is obtained using the following value iteration algorithm $\rho=1, \ldots, H$ :

$$
\begin{array}{r}
V_{\rho+1}^{(n)}\left(\pi^{(n)}, M\right)=\max \left\{M, R^{\prime}(n) \pi^{(n)}+\right. \\
\left.\beta \sum_{m=1}^{\left|M_{n}\right|} V_{\rho}^{(n)}\left(\frac{B^{(n)}(m) U^{(n)^{\prime}} \pi^{(n)}}{1_{\left|\mathbf{X}_{n}\right|}^{\prime} B^{(n)}(m) U^{(n)^{\prime}} \pi^{(n)}}, M\right) 1_{\left|\mathbb{X}_{n}\right|}^{\prime} B^{(n)}(m) U^{(n)^{\prime}} \pi^{(n)}\right\} .
\end{array}
$$

Here, $V_{H}\left(\pi^{(n)}, M\right)$ is the value function of an $H$-horizon dynamic programming recursion. Let $\gamma_{H}^{(n)}\left(\pi^{(n)}\right)$ denote the approximation Gittins index computed via the value iteration algorithm as follows:

$$
\gamma_{H}^{(n)}\left(\pi^{(n)}\right) \triangleq \min \left\{M: V_{H}^{(n)}\left(\pi^{(n)}, M\right)=M\right\}
$$

The finite-horizon Gittins index approximation $\gamma_{H}^{(n)}\left(\pi^{(n)}\right)$ of the above equation can be made arbitrarily accurate by choosing a sufficiently large horizon $H$. However, the value iteration recursion is not a directly practical solution methodology, since each iteration $V_{\rho}^{(n)}\left(\pi^{(n)}, M\right)$ needs to be computed over an uncountable set.

For any finite horizon, the value function of a POMDP is piecewise linear and converges $[8,143]$. Therefore, an elementary coordinate transformation is introduced to transform $V_{\rho}^{(n)}\left(\pi^{(n)}, M\right)$ to the value function of a standard POMDP. Each corresponding POMDP includes $2\left(\left|\mathbb{X}_{n}\right|+1\right)$ states, $\left(\left|\mathbb{M}_{n}\right|+1\right)$ observation states, and two actions $\{$ continue, retire $\}$. The near-optimal Gittins index $\gamma_{H}^{(n)}\left(\pi^{(n)}\right)$ for an arbitrary 
generation unit $n$ is given by the finite-dimensional representation [8]:

$$
\begin{aligned}
& \gamma_{H}^{(n)}\left(\pi^{(n)}\right)=\left\{M_{j}: \max _{\substack{\lambda_{i, H} \in \Lambda_{H}^{(n)}-\left\{\lambda_{1, H}\right\} \\
\lambda_{i, H}^{\prime}}}\left[\begin{array}{c}
\left(M_{j} / \bar{M}\right) \bar{\pi}^{(n)} \\
\left(1-M_{j} / \bar{M}\right) \bar{\pi}^{(n)}
\end{array}\right]-M_{j}=0\right\}, \\
& \text { where } \bar{\pi}^{(n)}=\left[\begin{array}{c}
\pi^{(n)} \\
0
\end{array}\right] \text {, and } \\
& M_{j}=\frac{\bar{M} \lambda_{j, H}^{\prime}(3) \pi^{(n)}}{\bar{M}+\left(\lambda_{j, H}^{\prime}(3)-\lambda_{j, H}^{\prime}(1)\right)^{\prime} \pi^{(n)}}, j=2, \ldots,\left|\wedge_{H}^{(n)}\right| \text {. }
\end{aligned}
$$

In the equation, $\lambda=\left[\begin{array}{llll}\lambda_{i, k}^{\prime}(1) & \lambda_{i, k}(2) & \lambda_{i, k}^{\prime}(3) & \lambda_{i, k}(4)\end{array}\right]^{\prime}$, where $\lambda_{i, k}(1), \lambda_{i, k}(3) \in \mathbb{R}^{\left|\mathbf{X}_{n}\right|}$, $\lambda_{i, k}(2), \lambda_{i, k}(4) \in \mathbb{R}$. The $2\left(\left|\mathbb{X}_{n}\right|+1\right)$-dimensional vectors $\lambda_{i, H}$ belong to pre-computable finite set of vectors $\Lambda_{H}^{(n)}$. Given the finite-dimensional representation of the Gittins index, several linear programming-based algorithms, such as Sondik's algorithm, Monahan's algorithm, Cheng's algorithm, and the Witness algorithm, can be used to compute vectors $\Lambda_{H}^{(n)}$.

However, the value iteration-based solution for computing the Gittins index only works for smart grids with a small number of generation units. For a large smart grid with a variety of generation units and associated power demand, the value iterationbased solution can become computationally intractable [8].

\subsubsection{Structural Results}

This subsection shows that for each generation unit and associated power demand in smart grids, with reasonable conditions on the cost vector $C$, the state transition probability matrix $U$ and the observation probability matrix $B$, the Gittins index can be monotone increasing in the information state (with respect to the monotone 
likelihood ratio (MLR) ordering [8]). This means that if the information states of these $N$ units and associated power demand at a given time instant are MLR comparable, the optimal policy is to pick the generation unit with the smallest information state with respect to the MLR ordering.

The following presents the conditions on the parameters $C, U$ and $B$ of an arbitrary generation unit and associated power demand, where its Gittins index $\gamma(\pi)$ is monotone in information state $\pi$ with respect to the MLR ordering. Assume that each generation unit and associated power demand includes the same states. Namely, $\mathbb{X}_{1}, \ldots, \mathbb{X}_{N}$ are equal to $\mathbb{X}$, and $\mathbb{M}_{1}, \ldots, \mathbb{M}_{N}$ are equal to $\mathbb{M}$.

Theorem 2 Consider the following assumptions for each generation unit and associated with power demand:

Assumption 4 Costs satisfy $C(i) \leq C(i+1)$.

Assumption 5 State transition probability matrix $U$ is totally positive of order 2 (TP2), i.e., all its second order minors are non-negative. That is, determinants

$$
\left|\begin{array}{ll}
u_{i_{1} j_{1}} & u_{i_{1} j_{2}} \\
u_{i_{2} j_{1}} & u_{i_{2} j_{2}}
\end{array}\right| \geq 0 \text { for } i_{2} \geq i_{1}, j_{2} \geq j_{1} .
$$

Assumption 6 Symbol probabilities satisfy $\left(b_{i, m}\right)_{m \in \mathbb{M}} \leq_{r}\left(b_{i+1, m}\right)_{m \in \mathbb{M}}$ for $i=$ $1, \ldots,|\mathbb{X}|-1$

Then the Gittins index $\gamma(\pi)$ of each unit is MLR increasing. Therefore, if the information states of the $N$ units combined with power demand are MLR comparable, then the optimal policy $\mu^{*}$ is to pick the unit with the smallest information state with respect to $M L R$ ordering at each time slot, namely, $a_{k}=\mu^{*}\left(\pi_{k}^{(1)}, \ldots, \pi_{k}^{(N)}\right)=$ $\arg \min \left(\pi_{k}^{(n)}\right), n \in\{1, \ldots, N\}$. 
When the structural results method is used to solve the above scheduling problem, off-line computation of Gittins index is not needed. Instead, only the information state of the chosen unit and associated power demand is calculated at each time slot, and is compared to other information states with respect to MLR ordering. The structural results method dramatically decreases the computation time, especially when there are a variety of units in smart grids.

\subsubsection{Constraints}

In the above, the scheduling problem is solved without considering constraints, such as security and transmission constraints. The requested power may overload critical transmission lines if the resulting schedule ignores these requirements. Therefore, the proposed scheme for unit commitment scheduling needs to consider various constrains during the scheduling process, such as transmission constraints, generator constraints and reserve constraints. Appropriate generation units are scheduled using the following algorithm. 
Algorithm 1: Unit commitment scheduling

1: Initialization.

2: Get each Gittins index of each generation unit at time $k$

3: Repeat:

4: Check each unit in the order of the increasing Gittins index to see if it meets the constraints

5: $\quad$ If yes, schedule the unit $j=j+1$;

6: Until $G$ units have been scheduled $(j=G)$.

\subsubsection{Simulation Results and Discussions}

In this section, computer simulations are used to evaluate the performance of the proposed scheme. The following simulation scenarios are considered. There are wind turbine generators (WTGs), solar power systems farm (SPSFs), and conventional fossil-fuel generation systems (FFGS) in smart grids. For WTGs and SPSFs, $100 \mathrm{~kW}$ generators are used to meet the power demand. For example, one of the options can be a Northwind 100 with rated electrical power of $100 \mathrm{~kW}$ at the wind speed $15 \mathrm{~m} / \mathrm{s}$. Its cut-in wind speed is $4 \mathrm{~m} / \mathrm{s}$ and the shut-down speed is $25 \mathrm{~m} / \mathrm{s}$. Each generator system includes two power supply level states $\{$ high, low $\}$, and there are two demand load states \{peak, off-peak\}. The state transition probability matrices for wind speed and cloud coverage have the following common characteristics [10, 144, 145]:

1. The highest probability occurs on the diagonal of the matrix. Thus, if the current average hourly wind speed or cloud coverage is known, the next hourly 
wind speed or cloud coverage will have as higher probability to remain in the same state.

2. All of the transition probabilities are around the diagonal. This means that transitions to a more distant state are scarce.

The wind speed state transition probability matrix, taken from [144], was calculated from the average frequency of transitions of real wind speed data gathered at Tangiers, Morocco. The state transition probability matrix of cloud coverage is defined following to $[10,146]$. Therefore, the power supply level state transition probability matrices of the WTG, SPSF and FFGS are as follows:

$$
J^{(1)}=\left(\begin{array}{cc}
0.756 & 0.244 \\
0.279 & 0.721
\end{array}\right), J^{(2)}=\left(\begin{array}{ll}
0.850 & 0.150 \\
0.150 & 0.850
\end{array}\right), J^{(3)}=\left(\begin{array}{cc}
1.000 & 0.000 \\
0.000 & 1.000
\end{array}\right) .
$$

The above probability matrices might be different depending on the locations of the generators and the associated weather conditions. Power demand loads are determined by two Poisson processes, one for peak hours and another for off-peak hours, where the inter-arrival times between two requests are exponentially distributed with a mean of 1 hour and 12 hours, respectively [147]. Assume the demand in each request is $100 \mathrm{kWh} / \mathrm{h}$. The corresponding power demand load state transition matrix is defined as:

$$
T=\left(\begin{array}{cc}
0.700 & 0.300 \\
0.400 & 0.600
\end{array}\right) \text {. }
$$

These demand load state transition matrices might be different depending on the power demand of these generators. Their observation matrics are defined as:

$$
B_{s}^{(n)}=B_{t}=\left(\begin{array}{cc}
0.900 & 0.100 \\
0.100 & 0.900
\end{array}\right) \text {, where } n=1,2,3 .
$$


The price of electricity differs for peak rate 9.9 cents $/ \mathrm{kWh}$ and off-peak rate 5.1 cents/kWh (from www.hydroottawa.com) and that of solar power is 80.2 cents/kWh (from fit.powerauthority.on.ca) in Ottawa, Canada. The price of wind power is set to 20.5 cents $/ \mathrm{kWh}$. The cost matrices are as follows in $\$ / \mathrm{h}: \quad C^{(1)}=(20.5,25.5,890.5,1.7), \quad C^{(2)}=(80.2,85.2,950.2,6.7), C^{(3)}=$ $(309.9,314.9,1075.1,26.1)$, which is the total cost in each state calculated using the cost model in subsection 4.2.1.4. The data shows that customer interruption cost has the most significant impact on the total cost. It means that renewable energy is a good option when the generated power can meet the power demand, since renewable energy can minimize greenhouse gas emissions. When the generated power from renewable energy is not enough, the FFGSs have to be scheduled in order to avoid blackouts. Cost matrices can be adjusted for different scenarios. For example, in most developing countries, pollutant emission costs are not considered.

In the simulation results, each cost element includes utility cost, customer interruption cost and pollutant emission cost, and each cost value is the averaged result of 1000 simulations.

\subsubsection{Performance Improvement}

Simulations are run to compare the costs of the proposed scheme, with and without considering the dynamic power demand loads, and an existing scheme in [148] that does not consider (hidden) Markov models for modeling renewable energy resources. Fig. 4.3 illustrates the total costs in dollars for the first 20 simulated hours (though the same process can be used to simulate days and years). The results show that the proposed scheme has a lower cost than the existing scheme. In the proposed scheme, considering the dynamic power demand load leads to a lower cost than not considering it. Since the state of a renewable energy resource is not fully observable 


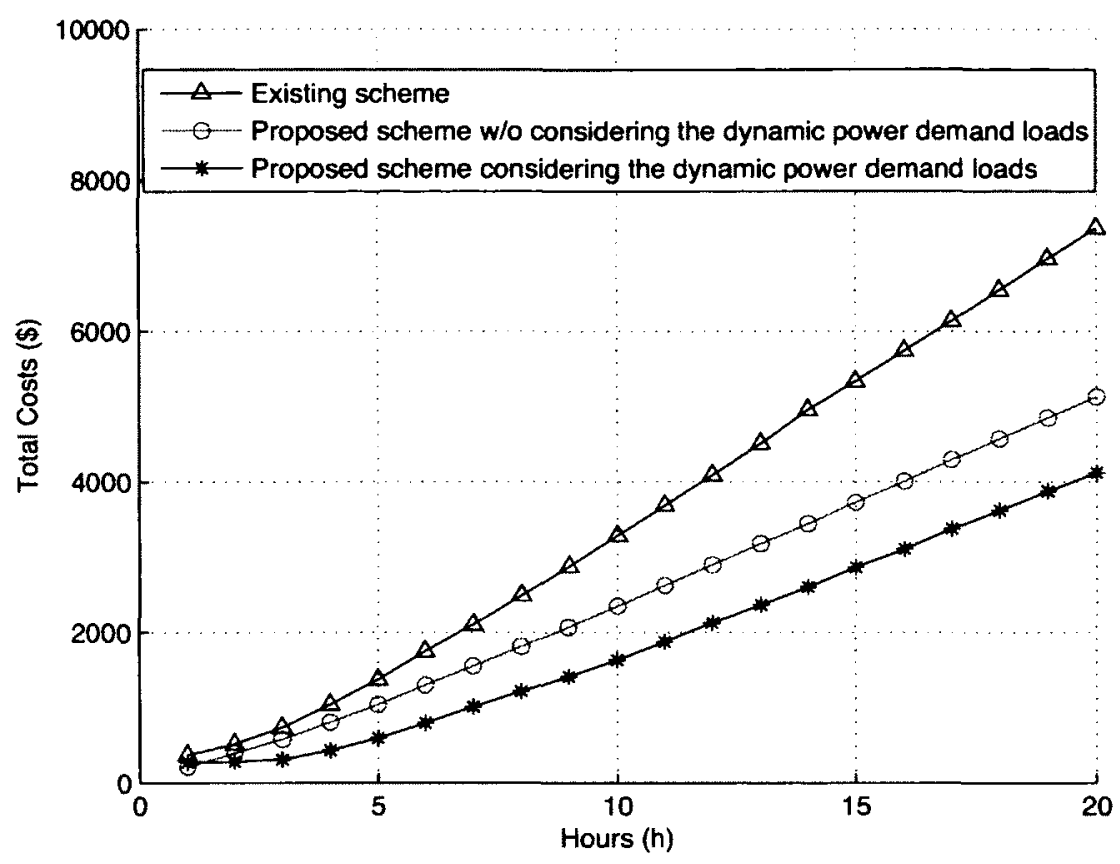

Figure 4.3: Total costs of the different schemes.

due to meteorological instability and complex system dynamics, a scheduler can make wrong decisions when hidden Markov models are not used. In our POMDP-based scheme, wrong decisions can be minimized by optimally scheduling these units, thus decreasing the total costs. The figure also shows that these three schemes perform similarly in the early stages, since the units and associated power demand are in a (high, peak) state, where the scheduling does not make a significant difference.

Various transition probabilities are also used in the simulations to evaluate the dynamic stability of the proposed scheme. Fig. 4.4 shows the average cost of these three schemes when the first component in the generation transition probability matrix varies from 0.7 to 1.0 . The results still show that the proposed scheme has the lowest cost among these three schemes. Fig. 4.4 also shows that the average cost decreases as the probability of each generator staying in the high state increases. When the generation transition probability reaches 1 , the average costs of these three 


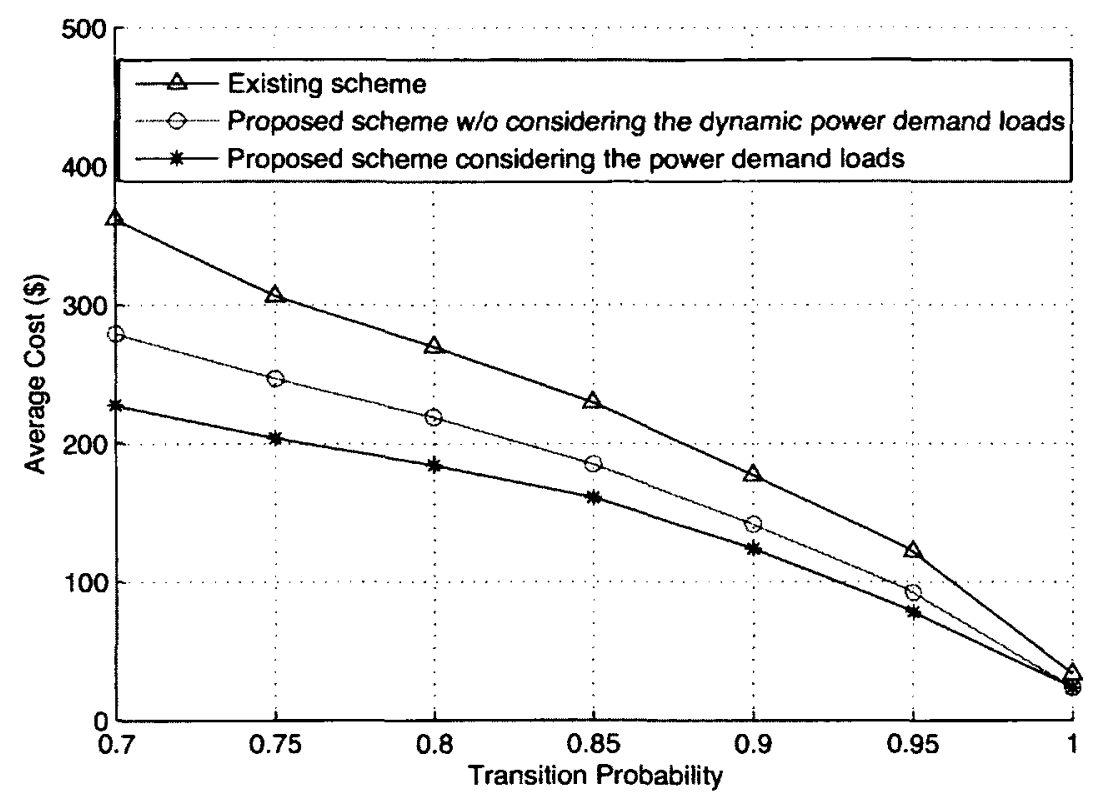

Figure 4.4: Average costs of three schemes under different transition probabilities.

schemes are very close, because all of the generator units stay in the high state, which means blackouts do not occur.

Different numbers of units are also used in the simulations to verify the scalability of the proposed scheme. Fig. 4.5 shows the average cost within the first 20 hours of the simulations of smart grids of different sizes. The same three kinds of units mentioned earlier are used in these simulations. The results show that the proposed scheme has the lowest cost compared to the existing scheme and the proposed scheme without considering the demand loads. The results also show that the cost of the proposed schemes and existing scheme decreases when the number of available units in smart grids increases from 3 to 18 . The reason is that there are more units that can be selected, so low-energy units can be avoided when demand is high. 


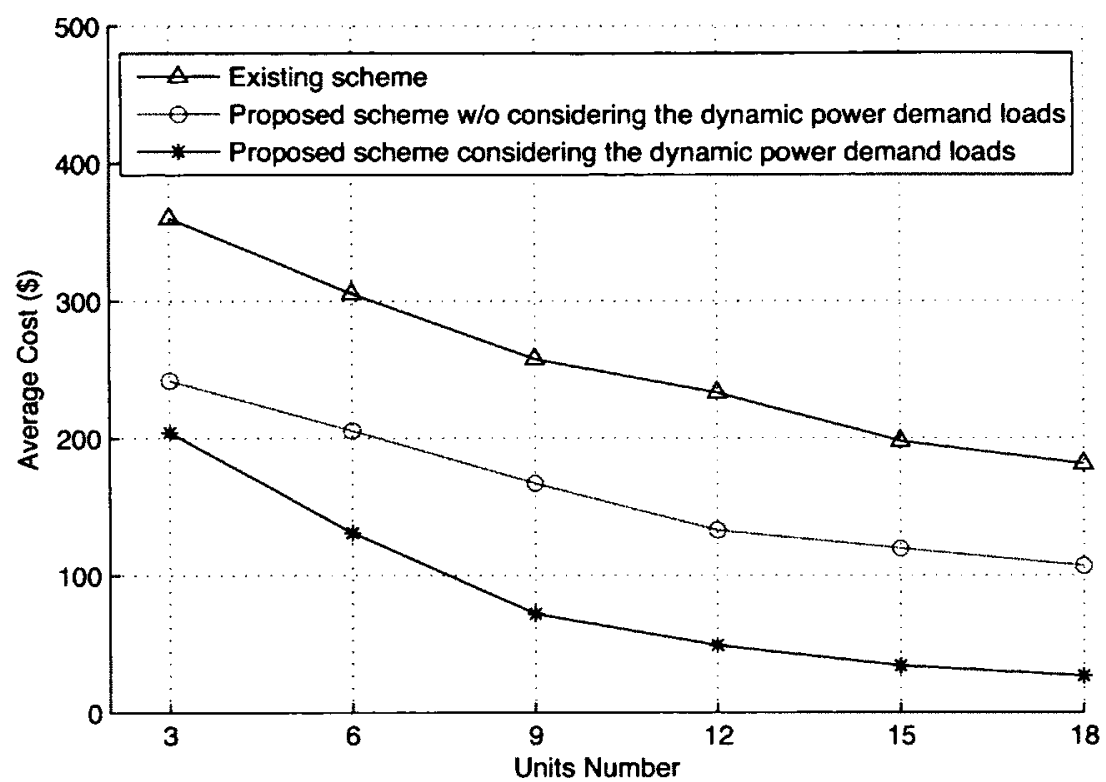

Figure 4.5: Average costs of three schemes with varying units in smart grids.

\subsubsection{Pollutant Emission Improvement}

Different values of pollutant emission costs are also used in the simulations to analyze how they affect the total costs and $\mathrm{CO}_{2}$ emissions in smart grids, since reducing pollutant emissions is the main motivation for employing renewable energy generation. The results are illustrated in Fig. 4.6, which shows that the total costs increase with the pollutant emission cost. When the pollutant emission cost is $\$ 0 / \mathrm{kWh}$, only conventional generation systems are scheduled in the system. The higher the pollutant emission cost, the greater the chance that renewable energy generators are scheduled. The levels of emissions resulting from the units are also compared in our simulation. The $\mathrm{CO}_{2}$ emission is set with $0.6999 \mathrm{~kg} / \mathrm{kWh}$ [149]. The results are shown in Fig. 4.7. The figure shows that $\mathrm{CO}_{2}$ emissions increase with the reduction of the pollutant emission costs. 


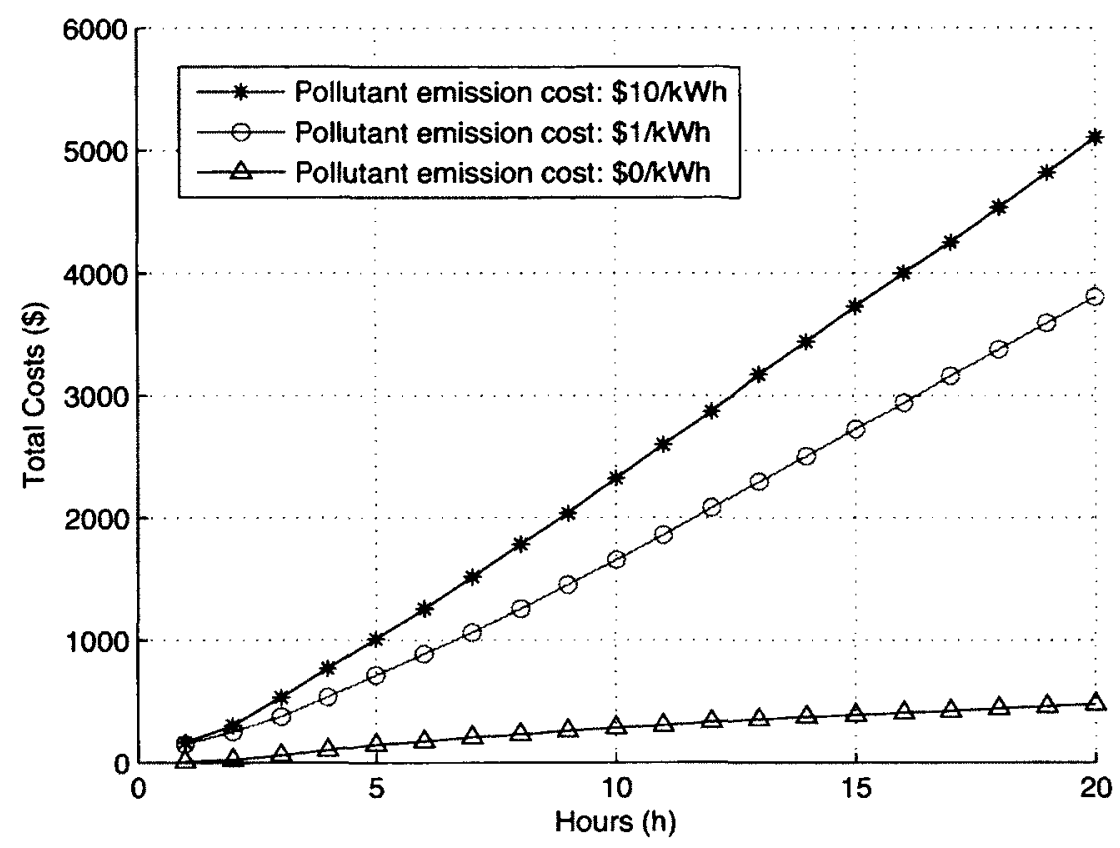

Figure 4.6: Total costs versus hours with various pollutant emission cost levels.

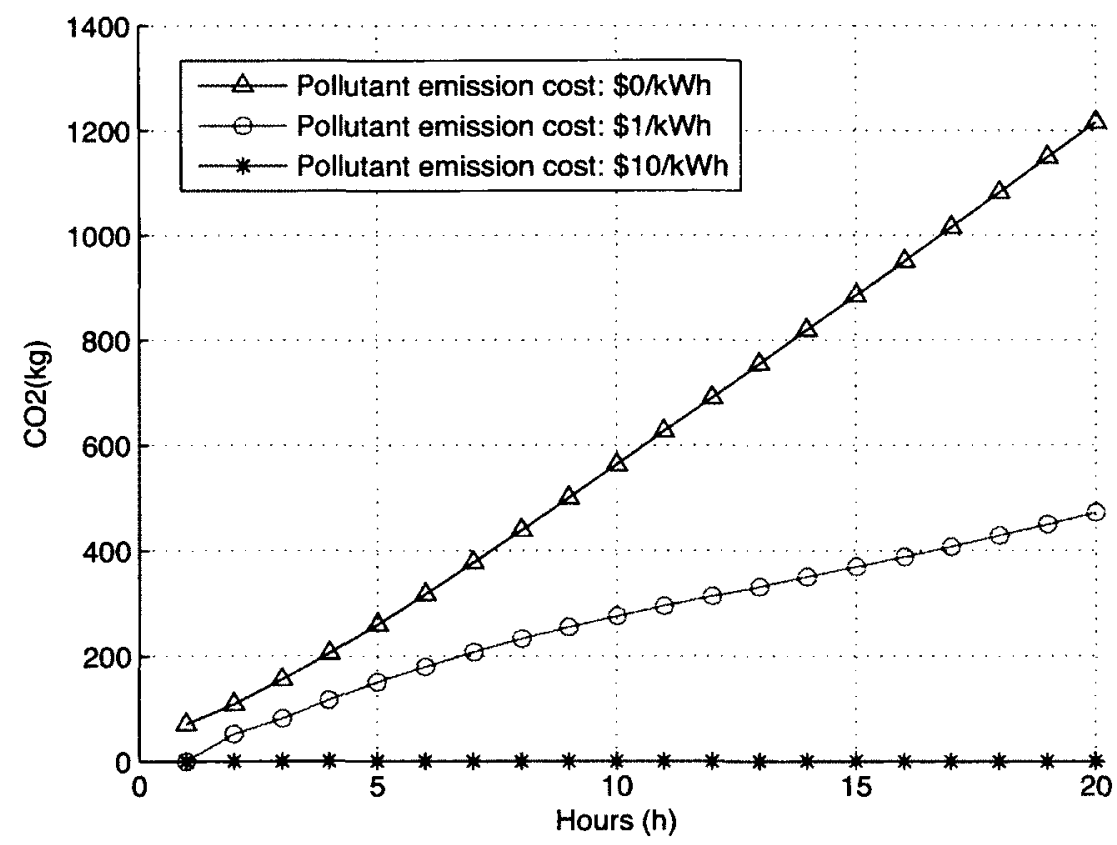

Figure 4.7: $\mathrm{CO}_{2}$ emissions versus hours with various pollutant emission cost levels. 
CHAPTER 4. STOCHASTIC SCHEDULING AND DECISION-MAKING . . 103

Table 4.1: The computation time of the two methods in the proposed distributed scheme.

\begin{tabular}{|c|l|l|l|l|}
\hline Method & $\begin{array}{l}2 \text { unit } \\
\text { types }\end{array}$ & $\begin{array}{l}4 \text { unit } \\
\text { types }\end{array}$ & $\begin{array}{l}20 \text { unit } \\
\text { types }\end{array}$ & $\begin{array}{l}50 \text { unit } \\
\text { types }\end{array}$ \\
\hline Structural results (off-line) & 0 & 0 & 0 & 0 \\
\hline Structural results (on-line) & $0.0427 \mathrm{~s}$ & $0.0576 \mathrm{~s}$ & $0.2337 \mathrm{~s}$ & $0.5960 \mathrm{~s}$ \\
\hline Value iteration algorithm (off-line) & $0.03 \mathrm{~s}$ & $8 \mathrm{~h} 1 \mathrm{~m} 22 \mathrm{~s}$ & unfeasible & unfeasible \\
\hline Value iteration algorithm (on-line) & $0.0379 \mathrm{~s}$ & $0.0531 \mathrm{~s}$ & - & - \\
\hline
\end{tabular}

\subsubsection{Comparisons of Computational Efficiency and Performance}

Simulations are performed to compare the computational efficiency in the proposed scheme using the two different methods. Table 4.1 shows the computation time spent in the proposed scheme in the off-line and on-line parts, as the total number of unit types in smart grids varies from 2 to 50 . For the value iteration algorithm, the on-line computation time is of the same level as that of the structural results method. The table also shows that the off-line time is the dominant part for the value iteration algorithm. The computation time dramatically increase when the number of unit types changes from 2 to 4 : from 0.03 seconds to more than 8 hours. In the structural results method, a quicksort algorithm with MLR ordering is used to sort the units by current information states. The off-line computation time for the structural results method is always equal to 0 , since the method is only used for on-line unit scheduling. The computation time of the structural results method slightly increase with the increasing type of the units in smart grids. This shows that the structural results are useful in practice in smart grids.

Simulations are also performed to compare the performance of the two methods. Fig. 4.8 shows that the total costs using the value iteration algorithm and those using the structural results method are very close to each other. 


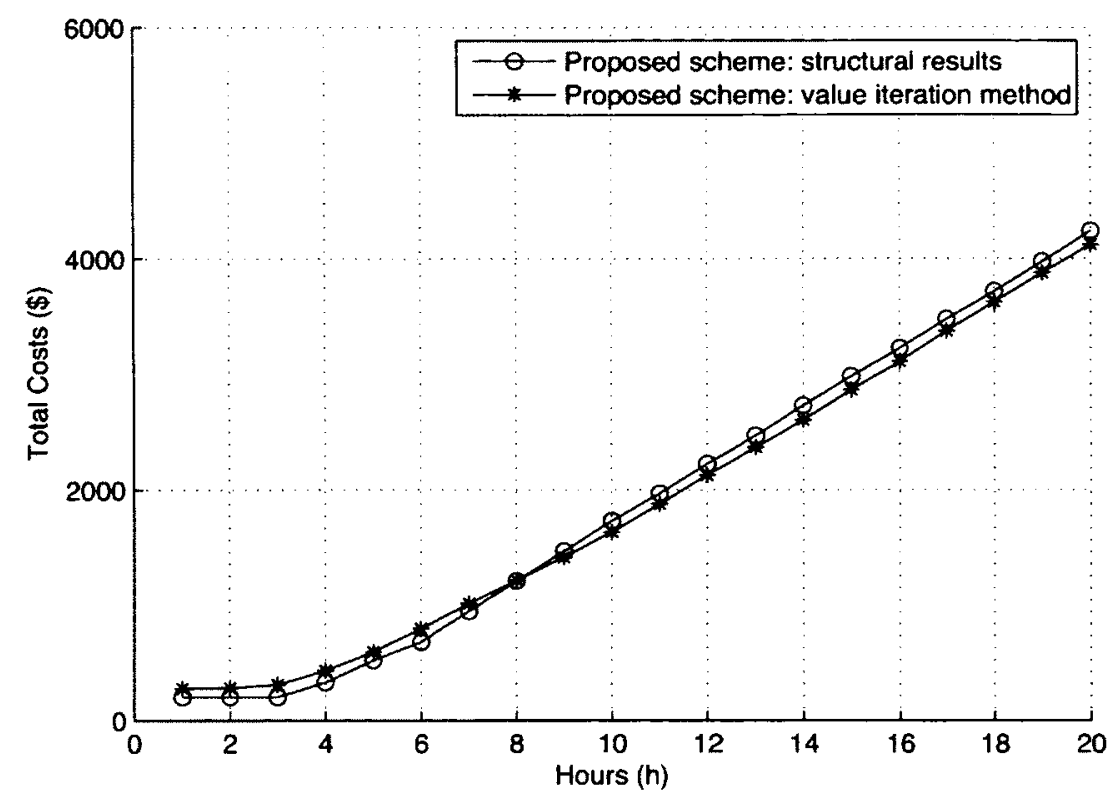

Figure 4.8: Total costs of the two methods in the proposed scheme.

\subsection{A Game-Theoretical Decision-Making Scheme for Electricity Retailers}

In this section, we propose a novel game-theoretical decision-making scheme for electricity retailers with real-time DSM in smart grids. This section is organized as follows. We model the interactions between the retailer and the customers as a fourstage Stackelberg game in Section 4.3.1. The Stackelberg game is analyzed through backward induction in Section 4.3.2. Simulation results are presented and discussed in Section 4.3.3.

\subsubsection{System Model}

In smart grids, an advanced metering infrastructure (AMI) and smart meters are needed to provide two-way real-time communications between retailers and customers. 
There are two major types of information flows in the network: control data is exchanged between the retailers and the customers, and monitoring and metering data is transmitted from the customers to the retailers.

In order to make efficient decisions, each retailer needs to learn customers' preferences and electricity consumption patterns. Therefore, each retailer can be equipped with several software tools: a database, a data-mining engine and a decision-making tool. A database stores the historical electricity consumption value of each customer. The data-mining engine is used to forecast the future consumption of each customer through his/her historical consumption data. The decision-making tool helps retailers to make market decisions. Various incentive programs can also be used by the retailers to encourage customers to provide their accurate electricity demand values and their electricity preferences in advance.

\subsubsection{Real-Time Electricity Demand Model for Customers}

In an electricity market, different electricity consumers require different levels of electricity, and might feel different level of satisfaction with the same price and amount of consumed electricity. In addition, a customer's satisfaction with the same level of electricity consumption can vary with time. The dynamic behaviors of customers can be accurately modeled by utility functions [150]. A utility function $u: \mathcal{X} \rightarrow \mathcal{R}$ represents a preference relation $\preceq$ on $\mathcal{X}$ iff for every $x, y \in \mathcal{X}, u(x)<u(y)$ implies $x \prec y$ ( $y$ is preferred to $x)$; $u(x)=u(y)$ implies $x \sim y$ ( $x$ and $y$ are equally preferred). The utility of a customer can be modeled by a quadratic function with the following two requirements [57]: First, it is a concave function of the electricity consumption. Second, the corresponding electricity demand function of each customer needs to be linear, in order to simplify the analysis of pricing. Therefore, the net utility function 
CHAPTER 4. STOCHASTIC SCHEDULING AND DECISION-MAKING ... 106

of an arbitrary user $i$ is defined as follows [57]:

$$
\mathcal{U}_{i}\left(p, d_{i}\right)=X_{i} d_{i}-\frac{\alpha_{i}}{2} d_{i}^{2}-p d_{i}
$$

where $X_{i}$ is a parameter that may vary among customers and at different times of the day, $d_{i}$ denotes the electricity consumption level of customer $i, \alpha_{i}$ is a pre-determined parameter, and $p$ is the price provided by the retailer.

Real-time pricing DSM is used by retailers, since it is an effective tool to guide and influence the electricity consumption behavior of customers [151]. Each customer adjusts his/her electricity consumption level in response to real-time electricity prices offered by the retailers to maximize its utility. The electricity consumption level of each customer can be calculated based on his/her utility function. We differentiate $\mathcal{U}_{i}\left(p, d_{i}\right)$ with respect to $d_{i}$ to attain his/her consumption function. Therefore, The electricity consumption function $\mathcal{D}_{i}(p)$ of customer $i$ can be expressed as follows:

$$
\mathcal{D}_{i}(p)=\frac{X_{i}-p}{\alpha_{i}} .
$$

If there are $|\mathcal{I}|$ users served by a retailer, where $|\mathcal{I}|$ is the cardinality of customer set $\mathcal{I}$, the total electricity demand is as follows:

$$
\sum_{i \in \mathcal{I}} \mathcal{D}_{i}(p)=\sum_{i \in \mathcal{I}}\left(\frac{X_{i}-p}{\alpha_{i}}\right)=F-G p
$$

In smart grids, bi-directional communications between a retailer and its customers make it possible to implement this real-time demand response. 


\subsubsection{Electricity Supply Sources for Retailers}

There are various electricity supply sources in an electricity market. Retailers need to make decisions about which kinds of electricity sources and how much electricity they procure. Electricity sources are divided into two types: Option I: cheaper but uncertain, and Option II: more expensive but certain. Without loss of generality, the cost of electricity from the energy sources is modeled by a linear function of the procured electricity. In Option I, the cost function can be defined as:

$$
\mathcal{C}(x)=C_{m} x,
$$

where $x$ denotes the electricity procured by the retailer and $C_{m}$ is the price for a unit of electricity. We assume that only $x \beta$ electricity received by the retailer, where $\beta$ $(0 \leq \beta \leq 1)$ is realization factor. This cost function can be used for modeling various electricity sources, such as self-production microgrids. In smart grids, various methods (such as electricity storage) have been used in the renewable energy generation or wholesale sides to increase the value of realization factor $\beta$. The cost function of Option II can be defined as:

$$
\mathcal{C}(x)=C_{g} x,
$$

where $x$ denotes the electricity procured by the retailer, and $C_{g}$ is the price for a unit of electricity. The above two cost functions are quite general and can be extended to the situations where the actual usage follows other empirical distribution functions.

\subsubsection{A Four-stage Stackelberg Game Model}

Each electricity retailer needs to make decisions about how much electricity to procure, from which sources, and what price to offer to customers in order to maximize 


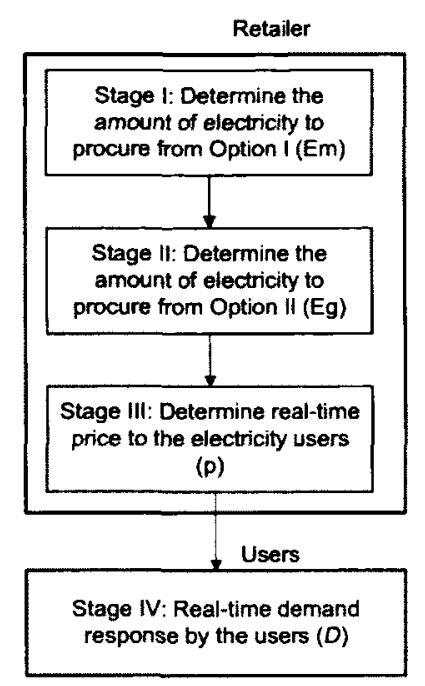

Figure 4.9: A four-stage Stackelberg game proposed for modeling the interactions between a retailer and its customers.

its profit. The customers adjust their electricity demands with the price offered by the retailer. We model and analyze the interactions between a retailer and its customers as a four-stage Stackelberg game illustrated in Fig. 4.9 as follows:

- Stage I: The electricity retailer, as the Stackelberg leader, first decides the amount of electricity $E_{m}$ procured from electricity source Option I with realization factor $\beta$.

- Stage II: The retailer then decides the amount of electricity $E_{g}$ procured from electricity source Option II, based on the received electricity level in stage I.

- Stage III: The retailer decides the real-time price $p$ to offer to the customers based on the total electricity supply.

- Stage IV: The customers, who are the followers in the Stackelberg game, adjust their individual electricity demand to maximize their individual utility.

It is optimal for the retailer to procure electricity first from electricity source Option I and then from electricity source Option II to maximize its profit. Therefore, 
the retailer should procure additional electricity from Option II, only if the electricity from Option I is not sufficient. This Stackelberg game model can be easily adjusted for different situations. We assume that the realization factor $\beta$ follows a uniform distribution in $[0,1]$. However, the main engineering insights are still held with arbitrary distributions of realization factor $\beta$.

In the following, we will analyze this four-stage Stackelberg game, and show how various system parameters affect the decisions.

\subsubsection{Four-Stage Stackelberg Game Analysis}

A common solution concept for a multi-stage Stackelberg game is the subgame perfect equilibrium (SPE) [152]. Backward induction, which captures the sequential dependence of the decisions in the stages of the game, is a general method to determine the SPE $[152,153]$. We first analyze how the customers adjust their individual electricity demand to maximize their utility based on the price offered by the retailer in stage IV. Then we analyze how the retailer makes real-time price decisions in stage III. We finally analyze the retailer's procurement decisions in stage II and in stage I.

\subsubsection{Real-Time Electricity Demand in Stage IV}

In this stage, customers determine their electricity demands given the unit price $p$ announced by the retailer in stage III, which has been explained in Subsection 4.3.1.1. We assume that the retailer knows individual customers' consumption patterns and preferences. 


\subsubsection{Optimal Real-Time Pricing Strategy in Stage III}

The retailer can only satisfy the electricity demand up to its supply. Therefore, the retailer's profit can be calculated as follows:

$$
\mathcal{R}\left(E_{m}, \beta, E_{g}, p\right)=\min \left(p \sum_{i \in \mathcal{I}} \mathcal{D}_{i}(p), p\left(E_{m} \beta+E_{g}\right)\right)-\left(E_{m} C_{m}+E_{g} C_{g}\right)
$$

In this stage, the retailer needs to determine the optimal price to offer the customers, considering the total demand in (4.17) and the total electricity supply received in stages I and II, in order to maximize the profit. Therefore, the largest possible profit in stage III can be calculated as follows:

$$
\mathcal{R}_{I I I}\left(E_{m}, \beta, E_{g}\right)=\max _{p \geq 0} \mathcal{R}\left(E_{m}, \beta, E_{g}, p\right)
$$

Maximizing the retailer's profit is the same as maximizing its revenue, since the amounts of electricity $E_{m}$ and $E_{g}$ are given and therefore the total cost $C_{m} E_{m}+C_{g} E_{g}$ is already fixed in this stage. Therefore, the optimal price should meet the following requirement:

$$
\max _{p \geq 0} \min \left(p \sum_{i \in \mathcal{I}} \mathcal{D}_{i}(p), p\left(E_{m} \beta+E_{g}\right)\right) .
$$

Let us define $D(p)=p \sum_{i \in \mathcal{I}} \mathcal{D}_{i}(p)$ and $S(p)=p\left(E_{m} \beta+E_{g}\right)$. There are three possible relationships between $D(p)$ and $S(p)$, depending on the total electricity supply $E_{m} \beta+E_{g}$. Three possible choices of $S(p)$ are described as $S_{j}(p)(j=1,2,3)$. The relationships are as follows:

1. $S_{1}(p)$ does not have intersection with $D(p)$, which is called an excessive supply regime.

2. $S_{2}(p)$ has only one intersection with $D(p)$, where $D(p)$ has a non-negative slope. 
This is also called an excessive supply regime.

3. $S_{3}(p)$ has only one intersection with $D(p)$, where $D(p)$ has a negative slope. This is called a conservative supply regime.

In the excessive supply regime, $\max _{p \geq 0} \min (S(p), D(p))=\max _{p \geq 0} D(p)$. In this regime, the total elect supply is higher than the total electricity demand at the optimal price. In the conservative supply regime, the revenue of the retailer is maximized at the unique intersection point of $D(p)$ and $S(p)$.

Theorem 3 The optimal electricity price and the corresponding maximum profit of the retailer in Stage III can be summarized in Table 4.2.

Proof 1 Taking the second derivative of $D(p)$, we get

$$
\frac{\partial^{2} D(p)}{\partial p^{2}}=-2 p<0
$$

Therefore, $D(p)$ is a concave function over $p$, and is maximized when $p=\frac{F}{2 G}$. $S(p)$ is linearly increasing function over $p$.

When $E_{m} \beta+E_{g}<\frac{F}{2}, S(p)$ intersects with $D(p) \cdot \min (D(p), S(p))$ is maximized at the intersection point, where price $p=\frac{F-E_{m} \beta-E_{g}}{G}$.

When $\frac{F}{2} \leq E_{m} \beta+E_{g} \leq F, S(p)$ intersects with $D(p) . \min (D(p), S(p))$ is maximized at the maximum value of $D(p)$, where price $p=\frac{F}{2 G}$.

When $E_{m} \beta+E_{g}>F, S(p)$ does not intersect with $D(p) \cdot \min (D(p), S(p))$ is maximized at the maximum value of $D(p)$, where price $p=\frac{F}{2 G}$.

\subsubsection{Optimal Electricity Procurement Strategy in Stage II}

In this stage, the retailer decides the amount of electricity $E_{g}$ procured from Option II given the amount of electricity $E_{m} \beta$ obtained in stage I to maximize its profit, 
Table 4.2: Optimal price decision and maximum profit of the retailer in Stage III.

\begin{tabular}{|l|l|l|}
\hline $\begin{array}{l}\text { Total Amount of } \\
\text { Electricity Obtained } \\
\text { in Stages I\&II }\end{array}$ & $\begin{array}{l}\text { Optimal Price } p * \\
\left(E_{m}, \beta, E_{g}\right)\end{array}$ & $\begin{array}{l}\text { Maximum Profit } \\
R_{I I I}\left(E_{m}, \beta, E_{g}\right)\end{array}$ \\
\hline $\begin{array}{l}\text { Excessive Supply: } \\
E_{m} \beta+E_{g} \geq \frac{F}{2}\end{array}$ & $p^{E S=\frac{F}{2 G}}$ & $\begin{array}{l}R_{I I}^{E S}\left(E_{m}, \beta, E_{g}\right)= \\
\frac{F^{2}}{4 G}-E_{g} C_{g}-E_{m} C_{m}\end{array}$ \\
\hline $\begin{array}{l}\text { Conservative Supply: } \\
E_{m} \beta+E_{g}<\frac{F}{2}\end{array}$ & $p^{C S=\frac{F-E_{m} \beta-E_{g}}{G}}$ & $\begin{array}{l}R_{I I I}^{C S}\left(E_{m}, \beta, E_{g}\right)= \\
\frac{\left(F-E_{m} \beta-E_{g}\right)\left(E_{m} \beta+E_{g}\right)}{G}- \\
E_{g} C_{g}-E_{m} C_{m}\end{array}$ \\
\hline
\end{tabular}

which can be described as follows:

$$
R_{I I}\left(E_{m}, \beta\right)=\max _{E_{g} \geq 0} R_{I I I}\left(E_{m}, \beta, E_{g}\right)
$$

The above problem can be decomposed into two following subproblems based on the two supply regimes in Table 4.2. The first subproblem is to choose $E_{g}$ such that the total electricity supply falls into the excessive supply regime in stage III, which can be described as follows:

$$
R_{I I}^{E S}\left(E_{m}, \beta\right)=\max _{E_{g} \geq \max \left\{\frac{F}{2}-E_{m} \beta, 0\right\}} R_{I I I}^{E S}\left(E_{m}, \beta, E_{g}\right)
$$

The second subproblem is to choose $E_{g}$ such that the total electricity supply falls into the conservative supply regime in stage III, which can be described as follows:

$$
R_{I I}^{C S}\left(E_{m}, \beta\right)=\max _{0 \leq E_{g} \leq \frac{F}{2}-E_{m} \beta} R_{I I}^{C S}\left(E_{m}, \beta, E_{g}\right)
$$

Theorem 4 The optimal amount of electricity to procure and the corresponding maximum profit of the retailer in stage II can be summarized in Table 4.3. 
CHAPTER 4. STOCHASTIC SCHEDULING AND DECISION-MAKING . . 113

Table 4.3: Optimal amount of electricity to procure and maximum profit in Stage II.

\begin{tabular}{|l|l|l|}
\hline Given $E_{m} \beta$ After Stage I & $\begin{array}{l}\text { Optimal Amount of } \\
\text { Electricity } E_{g}^{*} \text { Pro- } \\
\text { cured from Option II }\end{array}$ & Maximum Profit $R_{I I}\left(E_{m}, \beta\right)$ \\
\hline$(\mathrm{CS} 1) E_{m} \beta \leq \frac{F-G C_{g}}{2}$ & $E_{g}^{C S 1}=\frac{F-G C_{g}-2 E_{m} \beta}{2}$ & $\begin{array}{l}R_{I I}^{C S 1}\left(E_{m}, \beta\right)=\frac{F^{2}}{4 G}+\frac{G C_{g}^{2}}{4}- \\
\frac{F C_{g}}{2}+C_{g} E_{m} \beta-E_{m} C_{m}\end{array}$ \\
\hline$(\mathrm{CS} 2) E_{m} \beta \in\left(\frac{F-G C_{g}}{2}, \frac{F}{2}\right]$ & $E_{g}^{C S 2}=0$ & $\begin{array}{l}R_{I I}^{C S 2}\left(E_{m}, \beta\right)=\frac{\left(F-E_{m} \beta\right) E_{m} \beta}{G}- \\
E_{m} C_{m}\end{array}$ \\
\hline$(\mathrm{ES} 3) E_{m} \beta>\frac{F}{2}$ & $E_{g}^{E S 3}=0$ & $R_{I I}^{E S 3}\left(E_{m}, \beta\right)=\frac{F^{2}}{4 G}-E_{m} C_{m}$ \\
\hline
\end{tabular}

Proof $2 R_{I I I}^{E S}$ in (4.25) linearly decreases with $E_{g}$. Therefore, $R_{I I I}^{E S}$ is maximized at the lower bound of the set (i.e., $E_{g}^{*}=\max \left\{\frac{F}{2}-E_{m} \beta, 0\right\}$ ).

If $E_{m} \beta>\frac{F}{2}$, the obtained electricity from Option I at the first stage is already in the excessive supply regime. Therefore, it is optimal to not procure any electricity at this stage.

If $0 \leq E_{m} \beta \leq \frac{F}{2}$, the optimal profit in (4.26) is always greater than or equal to that in (4.25), and it is enough to only consider the conservative supply regime. Since

$$
\begin{aligned}
\frac{\partial R_{I I I}^{C S}\left(E_{m}, \beta, E_{g}\right)}{\partial E_{g}} & =\frac{F-2 E_{m} \beta-2 E_{g}}{G}-C_{g}, \\
\frac{\partial^{2} R_{I I I}^{C S}\left(E_{m}, \beta, E_{g}\right)}{\partial E_{g}{ }^{2}} & =-\frac{2}{G}<0 .
\end{aligned}
$$

Therefore, $R_{I I I}^{C S}$ is a concave function over $E_{g}$. If $0 \leq E_{m} \beta \leq \frac{F-G C_{g}}{2}$, the optimal amount of electricity procured from Option II is $E_{g}^{*}=\frac{F-G C_{g}-2 E_{m} \beta}{2}$. If $\frac{F-G C_{g}}{2} \leq$ $E_{m} \beta \leq \frac{F}{2}, E_{g}^{*}=0$.

Table 4.3 includes case CS1, case CS2, and case ES3 with different value sections of $E_{m} \beta$. The first two cases involve solving subproblem (4.26) in the conservative supply regime. The third case corresponds to the solution of subproblem (4.25) in 
the excessive supply regime. Although the procurement decisions of the retailer in cases CS2 and ES3 are the same, these two cases are listed separately since the retailer's profit in these two cases are different.

\subsubsection{Optimal Procurement Strategy in Stage I}

In this stage, the retailer needs to decide optimal amount of electricity $E_{m}$ to procure from Option I in order to maximize its expected profit by taking realization factor $\beta$ into account, which can be described as follows:

$$
R_{I}=\max _{E_{m} \geq 0} R_{I I}\left(E_{m}\right)
$$

where $R_{I I}\left(E_{m}\right)$ is the expected profit of the retailer in stage II. This above problem can be decomposed into the following three cases, based on the value of $E_{m}$.

In the first case (corresponds to case CS1 in Table 4.3), $E_{m}$ is less than or equal to $\frac{F-G C_{g}}{2}$, therefore $E_{m} \beta$ is always less than or equal to $\frac{F-G C_{g}}{2}$ for any value $\beta$. The expected profit of the retailer can be calculated as follows:

$$
\begin{aligned}
R_{I I}^{1}\left(E_{m}\right) & =E_{\beta \in[0,1]}\left[R_{I I}^{C S 1}\left(E_{m}, \beta\right)\right] \\
& =\frac{F^{2}}{4 G}+\frac{G C_{g}^{2}}{4}-\frac{F C_{g}}{2}+\left(\frac{C_{g}}{2}-C_{m}\right) E_{m}
\end{aligned}
$$

which is a linear function of $E_{m}$. If $C_{m}>\frac{C_{g}}{2}$, the expected profit linearly decreases with $E_{m}$. If $C_{m}<\frac{C_{g}}{2}$, the expected profit linearly increases with $E_{m}$.

In the second case, $E_{m}$ is within the set $\left(\frac{F-G C_{g}}{2}, \frac{F}{2}\right]$. Therefore, $E_{m} \beta$ can be in either case CS1 or CS2 in Table 4.3, depending on the value of $\beta$. The expected profit 
of the retailer can be calculated as follows:

$$
\begin{aligned}
R_{I I}^{2}\left(E_{m}\right) & =E_{\beta \in\left[0, \frac{F-G C_{g}}{2 E_{m}}\right]}\left[R_{I I}^{C S 1}\left(E_{m}, \beta\right)\right]+E_{\beta \in\left[\frac{F-G C_{g}}{\left.2 E_{m}, 1\right]}\right.}\left[R_{I I}^{C S 2}\left(E_{m}, \beta\right)\right] \\
& =\frac{\left(F-G C_{g}\right)^{3}}{24 G E_{m}}+\left(\frac{F}{2 G}-C_{m}\right) E_{m}-\frac{E_{m}^{2}}{3 G}
\end{aligned}
$$

The expected profit function in this case is a concave function, since its second-order derivative

$$
\frac{\partial^{2} R_{I I}^{2}\left(E_{m}\right)}{\partial E_{m}^{2}}=\frac{\left(F-G C_{g}\right)^{3}}{12 G E_{m}^{3}}-\frac{2}{3 G}<0
$$

as $E_{m}>\frac{F-G C_{g}}{2}$.

In the third case, $E_{m}$ is greater than $\frac{F}{2}$. Therefore, $E_{m} \beta$ can be in any of the three cases in Table 4.3, depending on the value of $\beta$. The expected profit of the retailer can be calculated as follows:

$$
\begin{aligned}
R_{I I}^{3}\left(E_{m}\right)= & E_{\beta \in\left[0, \frac{F-G C_{g}}{2 E_{m}}\right]}\left[R_{I I}^{C S 1}\left(E_{m}, \beta\right)\right]+E_{\beta \in\left[\frac{F-G C_{g}}{2 E_{m}}, \frac{F}{2 E_{m}}\right]}\left[R_{I I}^{C S 2}\left(E_{m}, \beta\right)\right] \\
& +E_{\beta \in\left[\frac{F}{2 E_{m}}, 1\right]}\left[R_{I I}^{E S 3}\left(E_{m}, \beta\right)\right] \\
= & \frac{-3 F^{2} C_{g}+3 F G C_{g}^{2}-G^{2} C_{g}^{3}}{24 E_{m}}+\frac{F^{2}}{4 G}-E_{m} C_{m} .
\end{aligned}
$$

The expected profit function in this case is a decreasing function, since the first-order derivative

$$
\frac{\partial R_{I I}^{3}\left(E_{m}\right)}{\partial E_{m}}=\frac{3 F^{2} C_{g}-3 F G C_{g}^{2}+G^{2} C_{g}^{3}}{24 E_{m}^{2}}-C_{m}<0,
$$

as $E_{m}>\frac{F}{2}$. Therefore, the expected profit function achieves maximum value when $E_{m}=\frac{F}{2}$. 
The expected profit of the retailer can be summarized as follows:

$$
R_{I I}\left(E_{m}\right)= \begin{cases}R_{I I}^{1}\left(E_{m}\right), & 0 \leq E_{m} \leq \frac{F-G C_{g}}{2} \\ R_{I I}^{2}\left(E_{m}\right), & \frac{F-G C_{g}}{2}<E_{m} \leq \frac{F}{2} \\ R_{I I}^{3}\left(E_{m}\right), & E_{m}>\frac{F}{2}\end{cases}
$$

Since the maximum value of the expected profit in the second case is always greater than the maximum profit in the third case, there is no need to consider the third case. This means that the retailer is either in case CS1 or case CS2 in stage II, and in the conservative supply regime in Stage III.

Theorem 5 The optimal procurement strategy of the retailer and its corresponding maximum expected profit in stage I can be summarized in Table 4.4 .

Proof 3 Table 4.4 includes two cases with different values of $C_{m}$ and $C_{g}$.

When $C_{m}>\frac{C_{g}}{2}$, the expected profit $R_{I I}\left(E_{m}\right)$ reaches its maximum when $E_{m}=0$, which means that it is optimal for the retailer not to procure any electricity from Option I in this stage. In this situation, the retailer's maximal expected profit $R_{I}^{H}$ only depends on the price of Option II.

When $C_{m} \leq \frac{C_{g}}{2}$, the highest expected profit $R_{I}^{L}$ can be calculated as follows:

$$
R_{I}=\max \left(\max \left(R_{I I}^{1}\left(E_{m}\right)\right), \max \left(R_{I I}^{2}\left(E_{m}\right)\right)\right)
$$

Since $R_{I I}^{1}\left(E_{m}\right)$ is an increasing linear function over $E_{m}, \max \left(R_{I I}^{1}\left(E_{m}\right)\right)$ is achieved when $E_{m}$ is equal to $\frac{F-G C_{g}}{2}$. For $R_{I I}^{2}\left(E_{m}\right)$, the optimal amount $E_{m}^{L *}$ is the solution to 
Table 4.4: Optimal procurement strategy and maximum expected profit in Stage I.

\begin{tabular}{|l|l|l|}
\hline & $\begin{array}{l}\text { Optimal Amount of } \\
\text { Electricity to Procure } \\
E_{m}^{*}\end{array}$ & $\begin{array}{l}\text { Maximum Expected } \\
\text { Profit } R_{I}\end{array}$ \\
\hline $\begin{array}{l}\text { High Electricity Price of } \\
\text { Option I: } C_{m}>\frac{C_{g}}{2}\end{array}$ & $E_{m}^{*}=0$ & $R_{I}^{H}=\frac{F^{2}}{4 G}+\frac{G C_{g}^{2}}{4}-\frac{F C_{g}}{2}$ \\
\hline $\begin{array}{l}\text { Low Electricity Price of Op- } \\
\text { tion I: } C_{m} \leq \frac{C_{g}}{2}\end{array}$ & $E_{m}^{*}=E_{m}^{L *}$ & $R_{I}^{L}$ in $(4.38)$ \\
\hline
\end{tabular}

the following equation:

$$
\frac{\left(G C_{g}-F\right)^{3}}{24 G E_{m}^{2}}+\frac{F}{2 G}-C_{m}-\frac{2 E_{m}}{3 G}=0
$$

if $E_{m}^{L *}$ lies in the interval of $\left[\frac{F-G C_{g}}{2}, \frac{F}{2}\right]$. Otherwise, $E_{m}^{L *}$ equals $\frac{F-G C_{g}}{2}$ or $\frac{F}{2}$, depending on the value of the corresponding maximum expected profit. Therefore, the retailer's maximum expected profit $R_{I}^{L}$ can be calculated as follows:

$$
R_{I}^{L}=\frac{\left(F-G C_{g}\right)^{3}}{24 G E_{m}^{L *}}+\left(\frac{F}{2 G}-C_{m}\right) E_{m}^{L *}-\frac{E_{m}^{L *} \times E_{m}^{L *}}{3 G} .
$$

We summarize the retailer's equilibrium decisions from stage I to stage III and the equilibrium electricity demand of the customers in Table 4.5. Several interesting observations about Table 4.5 are described as follows.

Observation 4.3.1 The optimal pricing $p^{*}$ is a non-increasing function in realization factor $\beta$.

When price $C_{m} \geq \frac{C_{g}}{2}$, optimal price $p^{*}$ is constant and independent of realization factor $\beta$. When $C_{m} \leq \frac{C_{g}}{2}$ and $\beta \leq \frac{F-G C_{g}}{2 E_{m}^{L^{*}}}$, optimal price $p^{*}$ is also constant and independent of realization factor $\beta$. When $C_{m} \leq \frac{C_{g}}{2}$ and $\beta>\frac{F-G C_{g}}{2 E_{m}^{L *}}$, optimal price decreases with the increase of realization factor $\beta$. 
Table 4.5: The retailer's and electricity customers' equilibrium behaviors.

\begin{tabular}{|l|c|c|c|}
\hline & High Price $C_{m} \geq \frac{C_{g}}{2}$ & \multicolumn{2}{|c|}{ Low Price: $C_{m} \leq \frac{C_{g}}{2}$} \\
\hline $\begin{array}{l}\text { Optimal Amount to } \\
\text { Procure from Option I } \\
E_{m}^{*}\end{array}$ & 0 & $E_{m}^{L *}$ solution to $(4.37)$ \\
\hline Realization Factor $\beta$ & $0 \leq \beta \leq 1$ & $0 \leq \beta \leq \frac{F-G C_{g}}{2 E_{m}^{L *}}$ & $\beta>\frac{F-G C_{g}}{2 E_{m}^{L *}}$ \\
\hline $\begin{array}{l}\text { Optimal Amount to } \\
\text { Procure from Option } \\
\text { II } E_{g}^{*}\end{array}$ & $\frac{F-G C_{g}}{2}$ & $\frac{F-G C_{g}}{2}-E_{m}^{L *} \beta$ & 0 \\
\hline $\begin{array}{l}\text { Optimal Pricing } p * \\
\text { Highest Expected } \\
\text { Profit } R_{I}\end{array}$ & $R_{I}^{H}=\frac{F^{2}}{4 G}+\frac{G C_{g}^{2}}{4}-\frac{F C_{g}}{2}$ & $\frac{R_{I}^{L} \text { in }(4.38)}{2 G}$ & $R_{I}^{L}$ in $(4.38)$ \\
\hline Customer $i$ 's Utility & $\frac{\left(2 G X_{i}-F-G C_{g}\right)^{2}}{8 G^{2} \alpha_{i}}$ & $\frac{\left(2 G X_{i}-F-G C_{g}\right)^{2}}{8 G^{2} \alpha_{i}}$ & $\frac{\left(G X_{i}-F+\beta E_{m}^{L *}\right)^{2}}{2 G^{2} \alpha_{i}}$ \\
\hline
\end{tabular}

Observation 4.3.2 The retailer will procure electricity from Option I only if price $C_{m}$ is lower than a threshold. Furthermore, the retailer will procure electricity from Option II only if the obtained electricity from Option I is below a threshold.

Observation 4.3.3 The retailer's highest expected profit always benefits from the availability of Option I when its electricity price is low, i.e. $C_{m} \leq \frac{C_{g}}{2}$.

\subsubsection{The Retailer's Realization Profit}

The retailer's profit, for a given realization factor $\beta$, is defined as realization profit. When the price provided by Option I is low (i.e., $C_{m} \leq \frac{C_{g}}{2}$ ), the retailer's realization profit can be obtained base on the value of $\beta$. When the price of electricity Option I is high (i.e., $C_{m}>\frac{C_{g}}{2}$ ), the realization profit equals its expected profit $R_{I}^{H}$ in Table 4.5 , since no electricity will be procured from Option I.

Theorem 6 The retailer's realization profit is a strictly increasing function in realization factor $\beta$ in the low cost regime. 
Proof 4 When $\beta$ is less than or equal to $\frac{F-G C_{g}}{2 E_{m}^{L_{*}^{*}}}$, the realization profit can be calculated as follows:

$$
R_{I I}^{C S 1}(\beta)=\frac{F^{2}}{4 G}+\frac{G C_{g}^{2}}{4}-\frac{F C_{g}}{2}-E_{m}^{L *} C_{m}+C_{g} \beta E_{m}^{L *}
$$

which is linearly increasing with $\beta$. When $\beta$ is greater than $\frac{F-G C_{g}}{2 E_{m^{*}}}$, the realization profit can be calculated as follows:

$$
R_{I I}^{C S 2}(\beta)=\frac{F E_{m}^{L *} \beta}{G}-\frac{\left(E_{m}^{L *}\right)^{2} \beta^{2}}{G}-E_{m}^{L *} C_{m}
$$

which is increasing with $\beta$, since the first-order derivative of the profit function is:

$$
\frac{\partial R_{I I}^{C S 2}(\beta)}{\partial \beta}=\frac{F E_{m}^{L *}}{G}-\frac{2\left(E_{m}^{L *}\right)^{2} \beta}{G}>0
$$

as $E_{m}^{L *}<\frac{F}{2}$.

\subsubsection{Simulation Results and Discussions}

In this section, we present simulation results to show the effectiveness of the proposed game-theoretical decision-making scheme and how the system parameters affect the decisions. In the simulations, there is one retailer serving electricity to ten customers. The parameters of the utility functions for these customers are set as follows: $X_{i}=$ $1(i \in\{1,2\}), X_{i}=2(i \in\{3,4,5\}), X_{i}=3(i \in\{6,7,8\}), X_{i}=4(i \in\{9,10\})$, and $\alpha_{i}(i \in[1,10])=2.5$.

We first vary the price of electricity in Option I and Option II, and study how this affects the decisions of the retailer in stage I. Fig. 4.10 shows that for a given price $C_{g}$ in Option II, the optimal amount $E_{m}^{*}$ is constant at the beginning and then decreases as price $C_{m}$ in Option I becomes higher, and drops to zero when $C_{m} \geq \frac{C_{g}}{2}$. Fig. 4.10 also shows that for a given price $C_{m}$, the optimal amount $E_{m}^{*}$ is non-decreasing with 


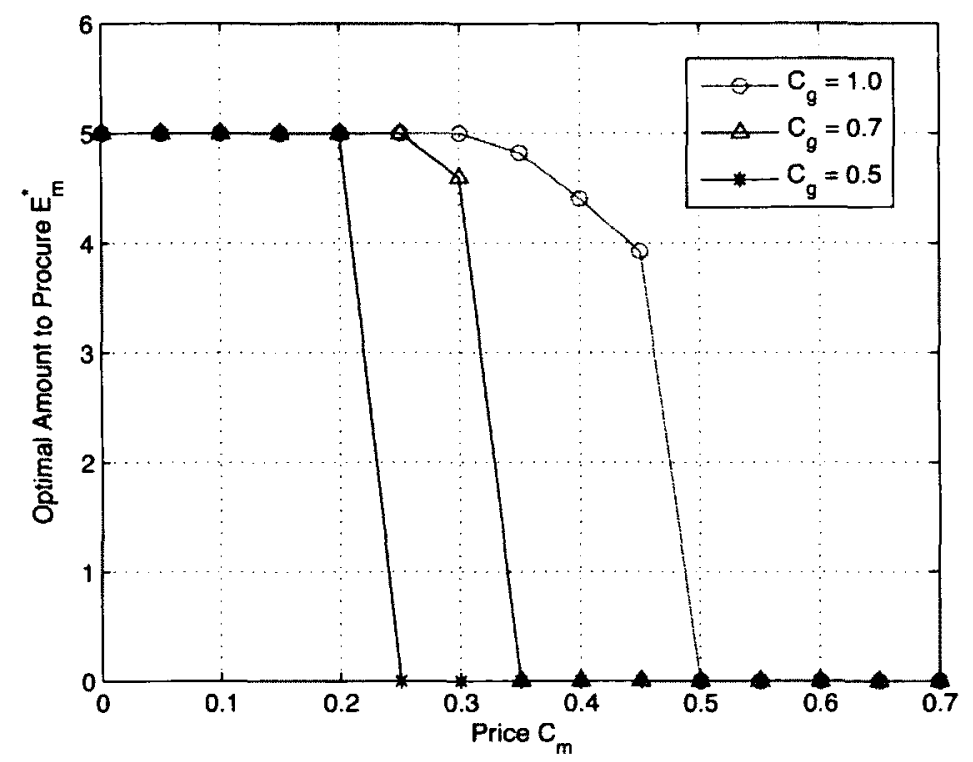

Figure 4.10: The optimal amount of electricity procured from electricity source Option I with the changes of prices $C_{m}$ and $C_{g}$.

the increase of price $C_{g}$, in which case procuring electricity from Option I becomes more attractive.

Next, we study how the decisions of the retailer in stage II are affected by price $C_{g}$ in Option II, and price $C_{m}$ and realization factor $\beta$ in Option I. When price $C_{m}$ is greater than or equal to half of price $C_{g}$, the optimal amount to procure from Option II only depends on price $C_{g}$. Therefore, we only simulate the situation when price $C_{m}$ is less than half of price $C_{g}$, corresponding to the low electricity price of Option I in Table III (denoted by "L"). Fig. 4.11 shows that the optimal amount $E_{g}^{*}$ procured from Option II decreases with the increase of realization factor $\beta$. The reason for this is that a higher value $\beta$ means more electricity is obtained from Option $I$, therefore there is a less need to procure electricity from Option II. The figure also shows that the optimal amount $E_{g}^{*}$ increases with the increase of price $C_{m}$ or the decrease of price $C_{g}$, in which case procuring electricity from Option II becomes more attractive.

We also investigate how the retailer's decisions in stage III change with price 


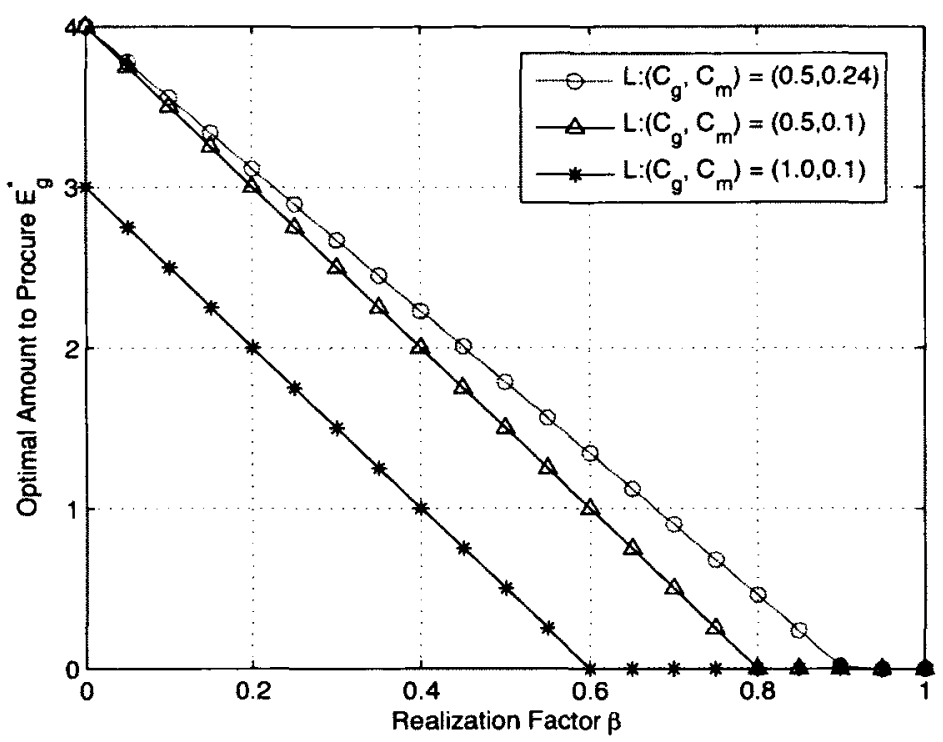

Figure 4.11: The optimal amount of electricity procured from Option II with the changes of price $C_{g}$, price $C_{m}$, and realization factor $\beta$ when $C_{m}<\frac{C_{g}}{2}$.

$C_{g}$, price $C_{m}$, and realization factor $\beta$. When price $C_{m}$ is greater than or equal to half of the price $C_{g}$, optimal price only depends on price $C_{g}$. Fig. 4.12 shows the simulation results when price $C_{m}$ is less than half of the price $C_{g}$. The figure shows that optimal price $p^{*}$ is a constant (i.e., $\frac{F+G C_{g}}{2 G}$ ) at the beginning, since the total amount of electricity obtained is $\frac{F-G C_{g}}{2}$. This constant increases with price $C_{g}$. When $\beta$ is larger than a threshold $\frac{F-G C_{g}}{2 E_{m}^{*}}$, optimal price $p^{*}$ decreases with the increase of the realization factor $\beta$. The threshold decreases with the increase of price $C_{g}$ or the decrease of price $C_{m}$.

We then study how the retailer's procurement decisions with/without considering Option I affect its highest expected profit $R_{I}$ with the change of price $C_{m}$. When $C_{m} \geq \frac{C_{g}}{2}$, expected profit does not depend on price $C_{m}$. Fig. 4.13 shows that when $C_{m}<\frac{C_{g}}{2}$, using our proposed decision-making scheme, the retailer achieves a higher expected profit than when only considering Option II. Fig. 4.13 shows the proposed decision-making scheme leads to a $1200 \%$ increase in profit when $C_{m}=0.2$. The 


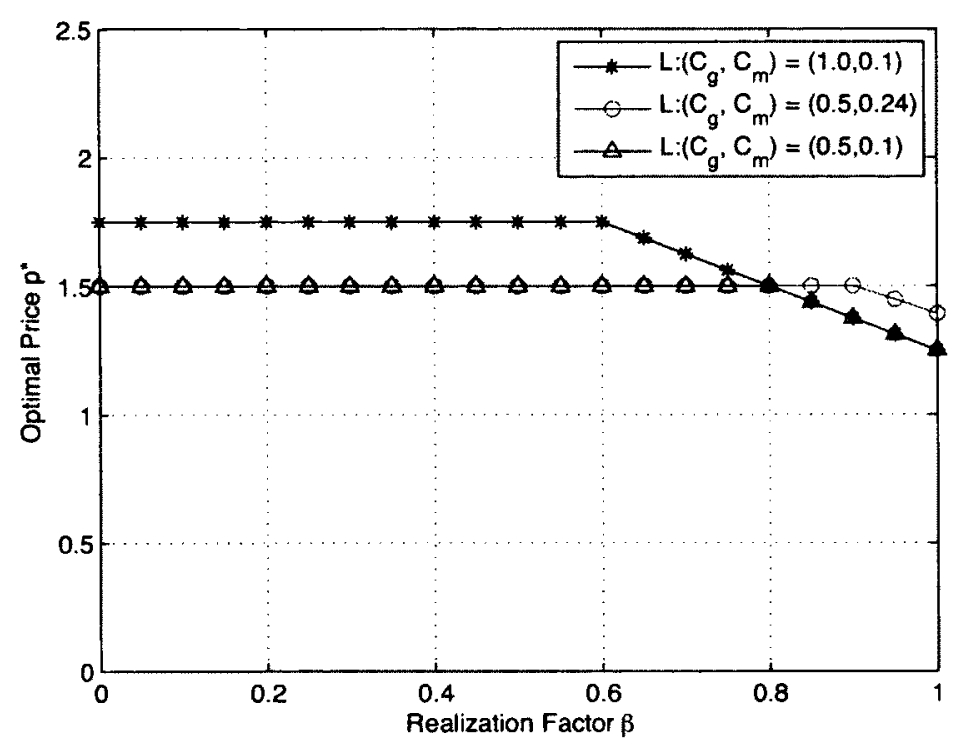

Figure 4.12: The optimal price offered to customers with the changes of price $C_{g}$, price $C_{m}$, and realization factor $\beta$ when $C_{m}<\frac{C_{g}}{2}$.

figure shows that the highest expected profit decreases with the increase of price $C_{m}$. When price $C_{m}$ equals half of price $C_{g}$, the retailer decides not to procure electricity from this option, and then the expected profit becomes the same as the profit without considering Option I.

We study how each user's utility is affected by price $C_{g}$, price $C_{m}$, realization factor $\beta$ and its characteristic parameters. When price $C_{m} \geq \frac{C_{g}}{2}$, each user's utility is constant with the increase of realization factor. Fig. 4.14 shows that for a user, the utility is constant at the beginning with the increase of realization factor $\beta$ when price $C_{m}<\frac{C_{g}}{2}$. The constant value decreases with price $C_{g}$ (i.e., the increase of optimal price $p *$ offered to the user). When $\beta$ is larger than $\frac{F-G C_{g}}{2 E_{m}^{L^{*}}}$ (decreasing with the value of price $C_{g}$ ), the user's utility increases, since optimal price $p^{*}$ offered by the retailer decreases with the increase of the realization factor $\beta$. The crossing situation shows that realization factor has more influence on users' utility when price $C_{m}$ is lower, and therefore a greater amount of electricity is procured from Option I. The figure 


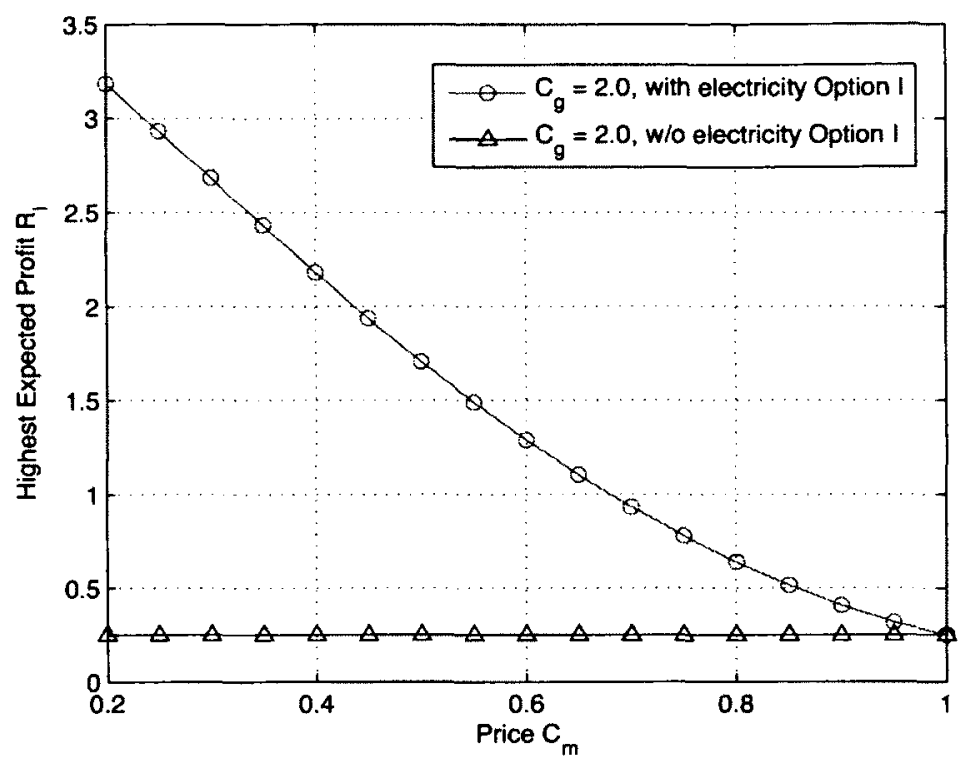

Figure 4.13: The highest expected profit with/without considering Option I with the change of price $C_{m}$ when $C_{m}<\frac{C_{g}}{2}$.

also shows that a user's utility also increases with the characteristic parameter $X_{i}$ in its utility function.

We also investigate how the variation of realization factor $\beta$ with time affects the optimal price $p^{*}$ offered by the retailer when price $C_{m}$ is less than half of price $C_{g}$. Fig. 4.15 and Fig. 4.16 show that even though the realization factor changes frequently over time, the corresponding optimal price is not necessarily changing. This result shows that the retailer does not need to change the price offered to the users at every moment, which makes it possible for the proposed decision-making scheme to be implemented in the real-world applications. Fig. 4.15 shows that the optimal price only changes in 12 out 50 time slots, with cost $C_{m}=0.98$, and cost $C_{g}=2.0$. The reason is that since cost $C_{m}$ is higher, the retailer does not procure large amount of electricity from Option I. As a result, the variability of $\beta$ has very small impact on the optimal price. Fig. 4.16 corresponds to the case where $C_{m}=0.70$, and $C_{g}=2.0$. As price $C_{m}$ is lower in Fig. 4.16, the retailer procures more electricity from Option 


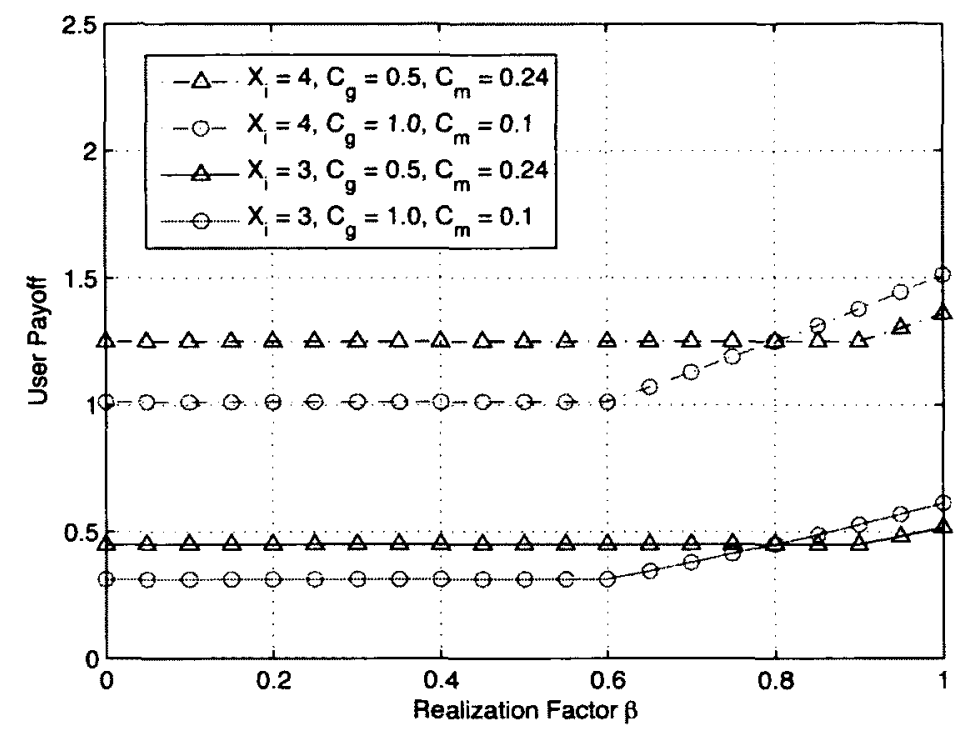

Figure 4.14: The utility of customer $i$ with the change of realization factor $\beta$ when $C_{m}<\frac{C_{g}}{2}$.

I, and the impact of $\beta$ on price is higher. Fig. 4.16 shows that the price changes in 28 out of 50 time slots.

We also check how optimal realization profit is affected by price $C_{g}$, price $C_{m}$ and realization factor $\beta$. Fig. 4.17 shows that the realization profit increases with $\beta$, if price $C_{m}$ is less than half of price $C_{g}$. The crossing feature of the two increasing curves shows the realization factor $\beta$ has a larger impact on the realization profit when price $C_{m}$ is low. When Option I is not considered in the procurement, the realization profit of the retailer is constant with the change of realization factor $\beta$. The figure also shows that using our proposed decision-making scheme, the retailer can achieve a higher realized profit than when only considering Option II when $\beta$ is higher than a certain level.

We finally investigate how the realization factor $\beta$ and prices $C_{g}$ and $C_{m}$ affect the realization utility of each user. Fig. 4.18 shows that the realization utility of a user is constant at the beginning, and then increases with $\beta$ since the optimal price offered 

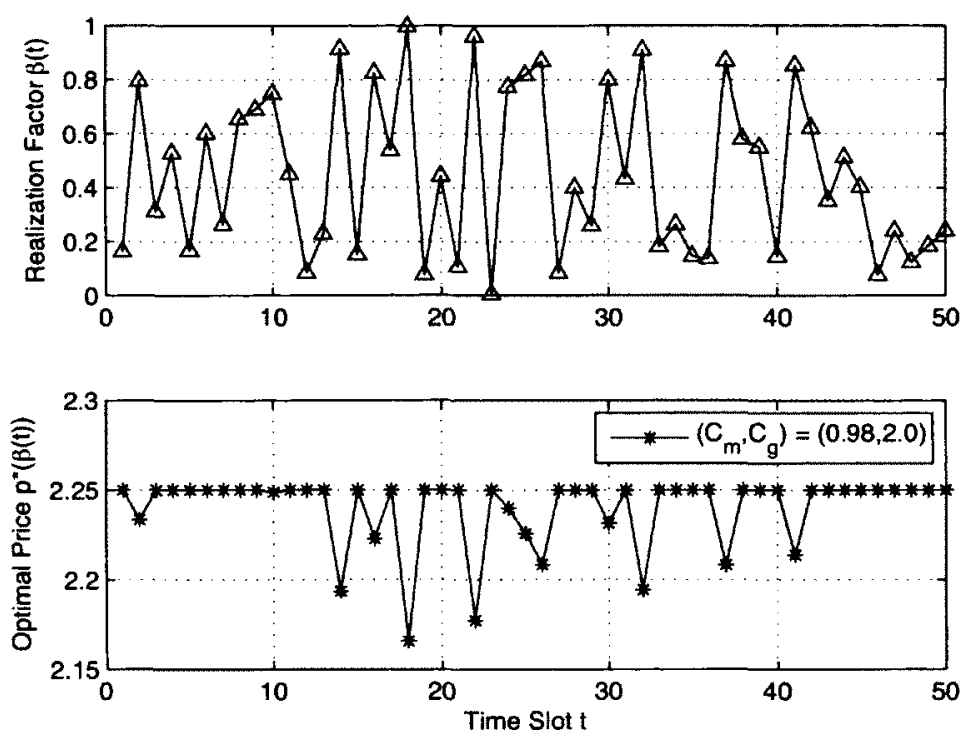

Figure 4.15: Optimal price with the variation of realization factor $\beta$, price $C_{m}=0.98$ and price $C_{g}=2.0$.
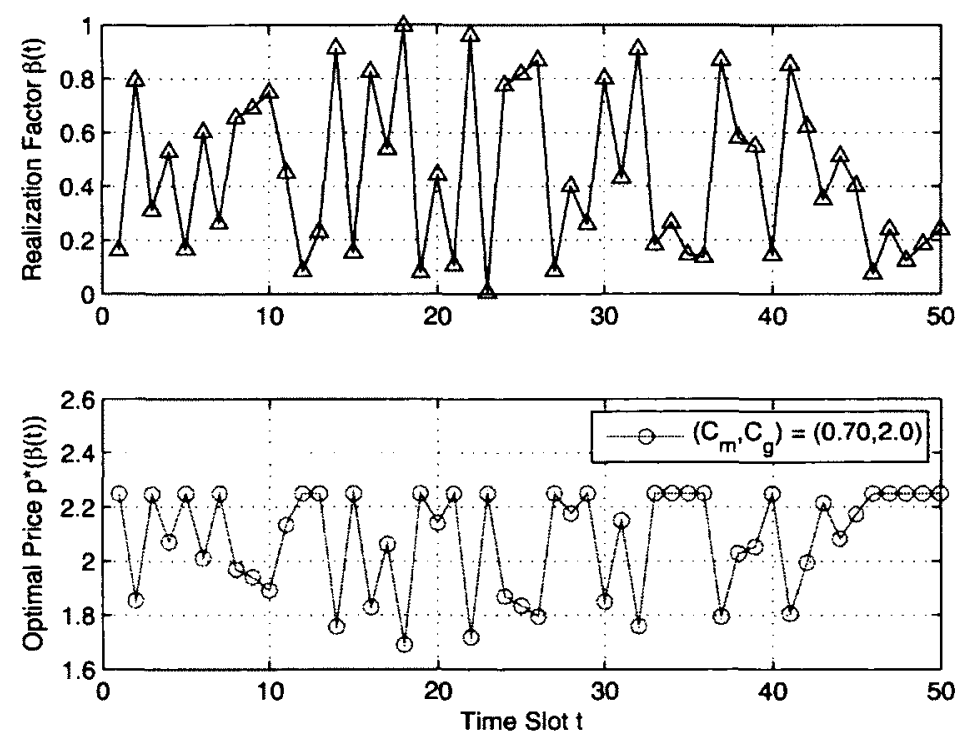

Figure 4.16: Optimal price with the variation of realization factor $\beta$, price $C_{m}=0.70$ and price $C_{g}=2.0$. 


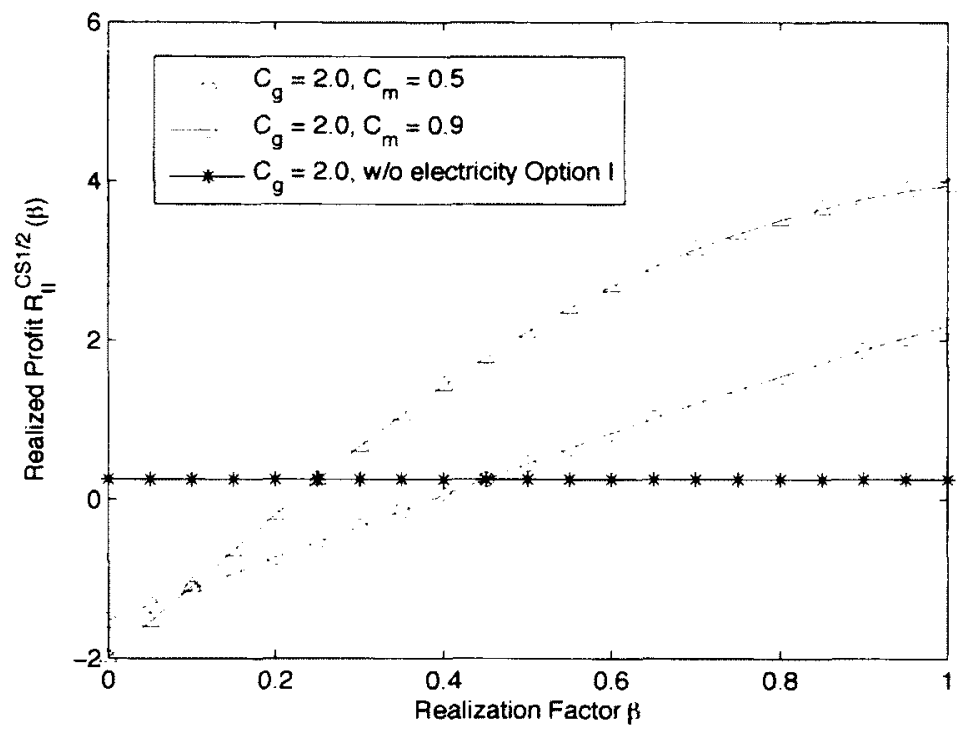

Figure 4.17: Realization profit with the change of price $C_{g}$, price $C_{m}$ and realization factor $\beta$ with/without considering Option I.

by the retailer starts to decrease. A lower $C_{m}$ provides a higher realization utility when the realized factor is high. The figure also shows the proposed decision-making scheme leads to a $430 \%$ increase in the user's realization utility when $C_{g}=2.0$ and $C_{m}=0.5$, compared to the scheme that does not consider Option I.

\subsection{Conclusion}

In this chapter, we have considered two important issues in smart grids: the UC scheduling problem, and the decision-making problem of electricity retailers. We have proposed a distributed stochastic UC scheduling scheme and a novel game-theoretical decision-making scheme for electricity retailers. In the UC scheduling scheme, the most suitable generation units are dynamically scheduled based on the intermittent renewable energy power generation situations, stochastic power demand loads, utility costs, reliability and pollution emissions of the generation units. Hidden Markov 


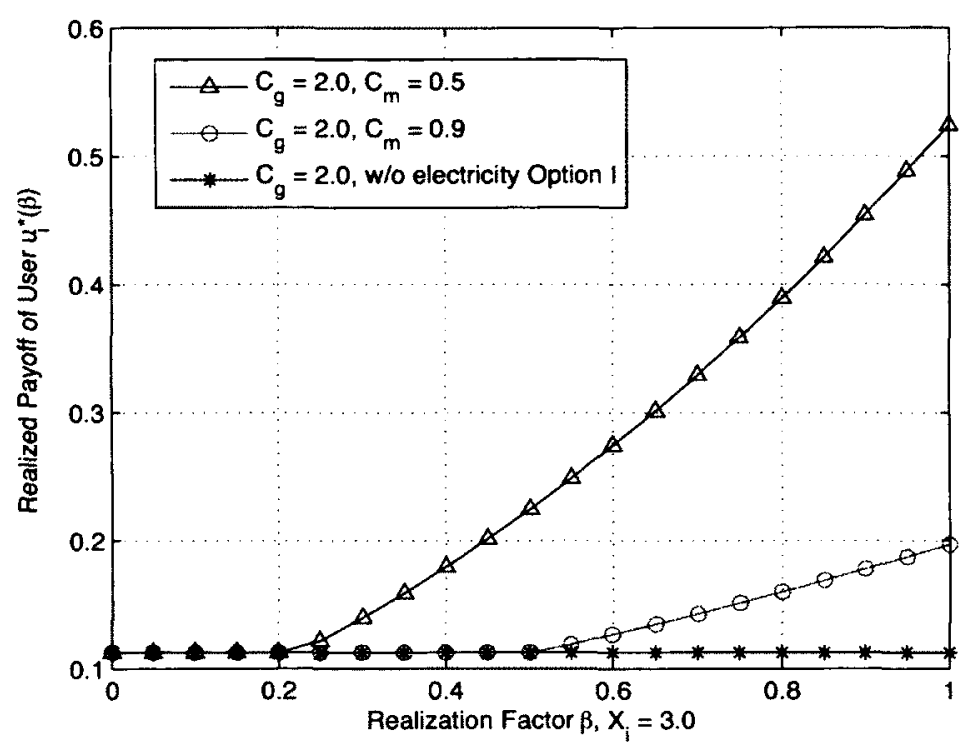

Figure 4.18: Realization utility of user $i$ with the change of realization factor with/without considering Option I. $X_{i}=3.0$.

models and a Markov-modulated Poisson process were used in modeling renewable energy resources and the power demand loads, respectively. The UC scheduling problem was formulated as a POMDP multi-armed bandit problem, and its optimal policy can be chosen using Gittins indices. A value iteration method and a structural results method were presented as possible solutions to the problem. In the game-theoretical decision-making scheme, the interaction between a retailer and its customers has been modeled as a four-stage Stackelberg game. The first three stages of the game analyze how the retailer should make optimal procurement and price decisions in order to maximize its profit. The fourth stage of the game shows how customers dynamically adjust their electricity demands with the price offered by the retailer to maximize their individual utility. Backward induction is used to determine the SPE of the fourstage Stackelberg game, since it captures the sequential dependence of the decisions in the stages of the game. Simulation results show that the proposed UC scheduling scheme can decrease the cost and greenhouse gas emissions. Using the proposed 
CHAPTER 4. STOCHASTIC SCHEDULING AND DECISION-MAKING _. 128

decision-making scheme, the retailer and customers can achieve a higher profit and higher utility, respectively. 


\section{Chapter 5}

\section{Dynamic Operations of Green Wireless Cellular Networks Powered by Smart Grids}

\subsection{Introduction}

In cellular networks, a base station consumes more than $90 \%$ of its peak energy even when there is little or no traffic [3]. To improve energy efficiency, base stations can dynamically coordinate, switching off redundant base stations during periods of low traffic. The deployment of heterogeneous networks based on smaller cells is an important technique to increase the energy efficiency of wireless cellular networks [83]. Cognitive radio will play an important role in improving energy efficiency for the future wireless cellular networks [94]. These two important techniques are combined to improve the energy efficiency of cellular networks. Since BSs are power hungry elements, absorbing them in smart grids can significantly increase power efficiency [83]. Therefore, smart grids are also considered in our proposed schemes in order to realize a "green" cellular network.

The highlights of the research contribution in Section 5.2 are as follows: 
- The dynamic operation of cellular base stations depends on the traffic, realtime price provided by smart grids, and the pollutant level associated with the generation of the electricity.

- Coordinated multipoint (CoMP) communication is used to ensure acceptable service quality in the cells whose base stations have been shut down to save energy.

- The active base stations decide on which retailers to procure electricity from and how much electricity to procure considering the pollutant level of each retailer and the price offered by the retailer.

- We formulate the system as a Stackelberg game, which has two levels: a cellular network level and a smart grid level. The close-form solutions to the proposed scheme are given.

The highlights of the research contribution in Section 5.3 are as follows:

- Real-time pricing demand-side management (DSM) is used in smart grids. Therefore, multiple retailers provide the real-time electricity prices to the heterogeneous network, and the femtocell base stations (FBSs) and the macro base station (MBS) adjust the amount of electricity they consume by performing energy-efficient power allocation. We assume that the electricity cost for each retailer includes the electricity procurement cost, and other costs, such as $\mathrm{CO}_{2}$ tax fees if the procured electricity is produced by non-renewable energy generators. We use a homogeneous Bertrand game with asymmetric costs to model price decisions made by the electricity retailers.

- Femtocells and macrocells share spectrums, and the cross-tier interference greatly restrict the network performance. To mitigate the interference effect 
and guarantee the quality of service (QoS), an interference price is proposed to allow the MBS to protect itself by charging the femtocell users.

- We formulate the problems of electricity price decision, energy-efficient power allocation and interference price in smart grids and the heterogeneous network as a three-level Stackelberg game. Stackelberg game has been successfully used in relay selection and power allocation problems in cooperative communication networks [154], among other areas. A backward induction method is used to analyze this Stackelberg game. Then an iteration algorithm is proposed to obtain the Stackelberg equilibrium solution.

Simulation results show that smart grids have an significant impact on green wireless cellular networks, and our proposed schemes can significantly reduce operational expenditure and $\mathrm{CO}_{2}$ emissions in green wireless cellular networks.

The work presented in this chapter has resulted in two journal papers $[155,156]$ (one accepted and another submitted), one conference paper [157] and one book chapter [158]. Possible future work in this area is provided in Chapter 6.

\subsection{Dynamic Base Station Operations in Cellular Networks with CoMP Communications}

In this section, we will discuss how the dynamic operation of cellular base stations based on the traffic arrival rate, real-time price provided by smart grids, and pollutant levels of electricity retailers can reduce operational expenditure and $\mathrm{CO}_{2}$ emissions in green wireless cellular networks. This section is organized as follows. We describe the system models in Section 5.2.1. The problem is formulated as a two-level Stackelberg game in Section 5.2.2. The proposed game is analyzed in Section 5.2.3. Simulation 


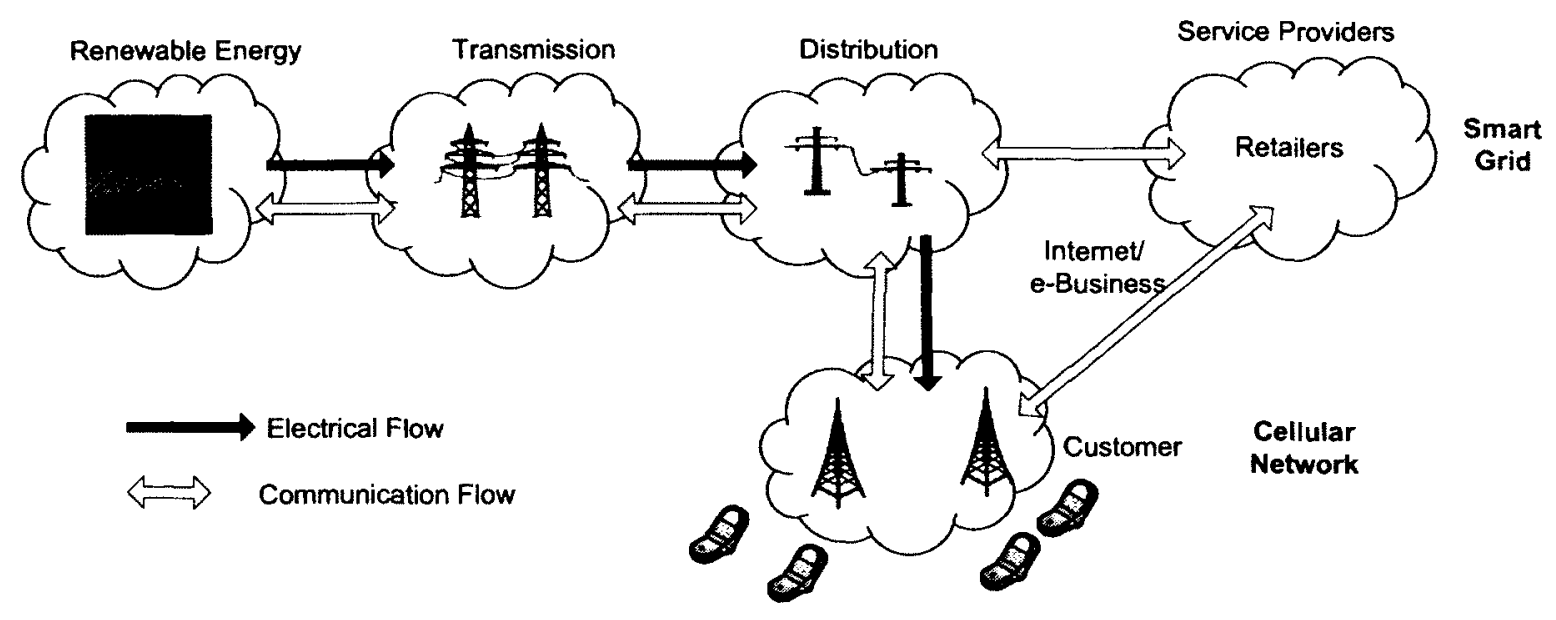

Figure 5.1: A cellular network powered by smart grids.

results are presented and discussed in Section 5.2.4.

\subsubsection{System Models}

We consider a cellular network powered by smart grids, as shown in Fig. 5.1. In this section, we first introduce CoMP communication. We then present service blocking probability model. Finally, we present the electricity consumption model for base stations.

\subsubsection{CoMP Communications}

In cellular networks, interference exists between inter-cells, which affects spectral efficiency, especially in urban cellular systems. CoMP, which is originally proposed to overcome this limitation, has been selected as a key technology for LTE-A [159]. CoMP can significantly improve average spectral efficiency and also increase the cell edge and average data rates.

In our system, redundant base stations are turned off when the traffic is low, the real-time price is too high, or the pollutant level of the electricity retailer is 

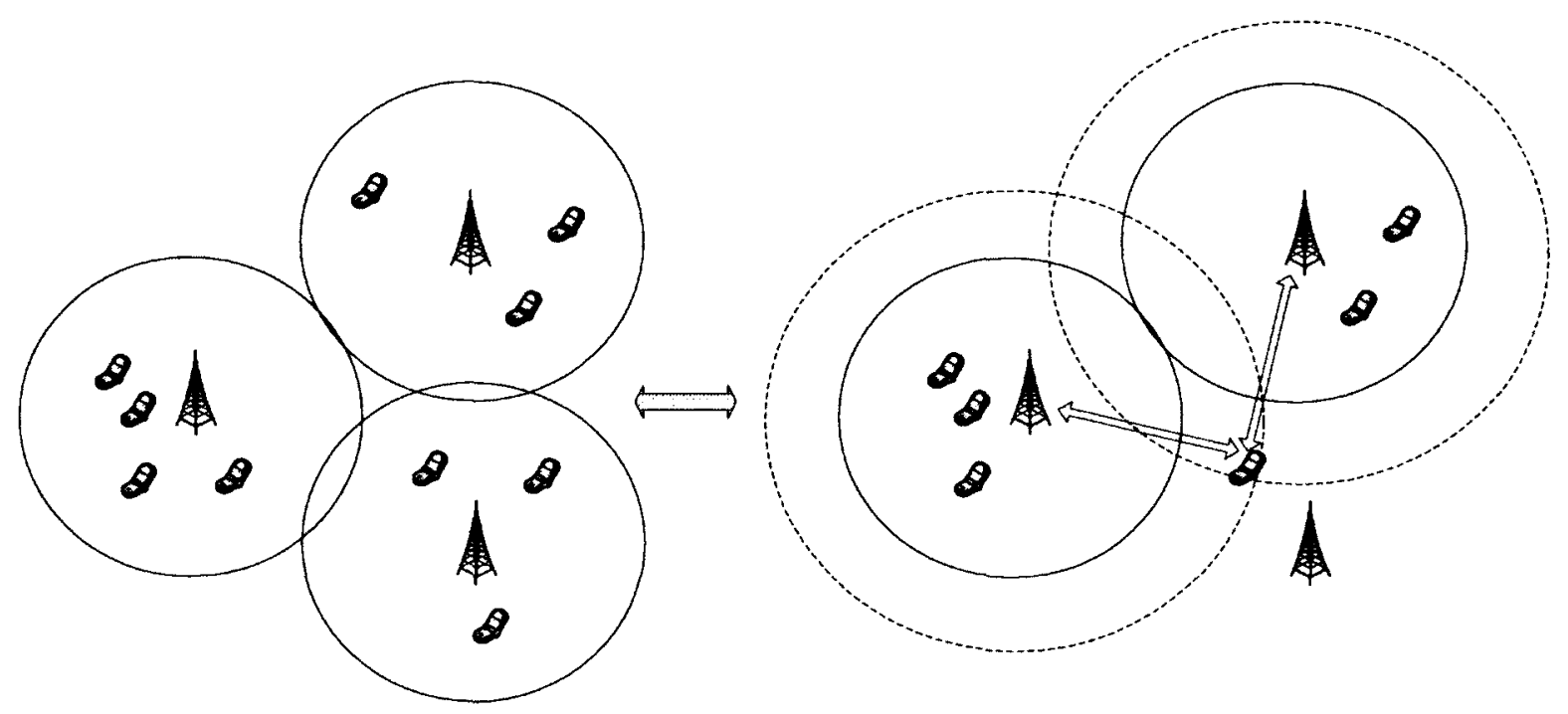

Figure 5.2: Coverage extension and energy saving with CoMP.

too high. CoMP is used among the active base stations to guarantee the coverage requirements [159]. In the downlink, the neighboring active base stations transmit cooperatively, and thus their coverage increases. In the uplink, multiple active base stations receive in coordination, which effectively reduces the requirement of received signal power at each individual base station. Base station coverage extension using CoMP is illustrated in Fig. 5.2. Together, CoMP can provide coverage for mobile users in nearby cells whose base station has been shut down during low activity period, and ensure the service blocking probability is within certain acceptable level.

In order to achieve the theoretical maximum capacity, all of the base stations in the network should cooperate with each other in each transmission or reception process. However, the introduced complexity is not acceptable in real systems. Therefore, standards have specified the maximal number of BSs that may cooperate with each other [160]. We assume that there are $i$ BSs in each CoMP cooperation cluster. All the possible combinations of the BSs in the cluster are denoted as the coordination set $\Theta$, whose cardinality is $2^{i}$. The base stations in the cluster can be switched on in 
any combination, where each combination set $\Psi$ is an element of set $\Theta$.

\subsubsection{Service Blocking Probability Model in the Cellular Network}

For an arbitrary element $\Psi \in \Theta$, the uplink sum-capacity for the cluster $C(\Psi)$ can be calculated as follows [161]:

$$
C(\Psi)=\log _{2} \operatorname{det}\left(\mathbf{I}_{|\Psi|}+P \mathbf{H H}^{\dagger}\right)
$$

where $\mathbf{I}_{|\Psi|}$ denotes a $|\Psi| \times|\Psi|$ identity matrix, $P$ denotes the transmission power of each user terminal, $\mathbf{H} \in \mathbb{C}^{|\Psi| \times|\Psi|}$ denotes the channel matrix. The number of channels available in the cluster $m$ can then be calculated as follows:

$$
m=\left\lfloor\frac{B C(\Psi)}{B_{0}}\right\rfloor
$$

where $B$ denotes the bandwidth allocated for each CoMP cluster, $B_{0}$ denotes the effective bandwidth required for a service. We assume that traffic characteristics, the desired packet-level QoS (e.g., packet delay and loss) guarantees, and the scheduling can together be represented by this effective bandwidth. Techniques for computing this effective bandwidth for different QoS requirements and traffic characteristics can be found in $[162,163]$. Therefore, the service blocking probability in the cluster $\mathcal{P}_{b}$ can be calculated according to Erlang-B formula [164]:

$$
\mathcal{P}_{b}=\frac{\frac{E^{m}}{m !}}{\sum_{j=0}^{m} \frac{E^{j}}{j !}}
$$

where $E=\lambda / \mu$. Here, $\lambda$ denotes the traffic arrival rate in the cluster, and $\mu$ denotes the average service rate. We define the relationship between the service blocking 
probability and a combination set as: $\mathcal{P}_{b}=\mathcal{D}(\Psi)$, where $\mathcal{D}(\Psi)$ is constructed by combining (5.1), (5.2), and (5.3).

\subsubsection{Electricity Consumption Model for Base Stations}

The energy consumption of each active cellular base station $P_{B S}$ includes three parts: electricity consumption due to the transmission power, signal processing, and backhauling, which can be denoted as follows [165]:

$$
P_{B S}=a P_{t x}+b P_{s p}+c P_{b h}
$$

where $P_{t x}, P_{s p}, P_{b h}$ denote the radiated power per base station, the signal processing power per base station, and the power due to backhauling per base station, respectively. $a, b$ and $c$ denote the scale factor associated with the corresponding power type, such as cooling and battery backup.

The transmit power of each base station depends on the path loss in the propagation channel. Therefore, the average transmit power per base station can be denoted as follows [165]:

$$
\log \left(P_{t x}\right)=\log P_{\min }-\log K+\phi \log (D / 2)
$$

where $P_{\min }$ denotes minimum receiving power required by user terminal, $K$ is a parameter accounting for effects including BS antenna settings, carrier frequency and propagation environment, $\phi$ is the path loss exponent, and $D$ denotes the inter site distance.

The energy consumption due to signal processing operations is partly determined by the employed air interface as well as the amount of cooperation between base stations. The uplink channel estimation operation consumes around $10 \%$ of the overall signal processing power [165]. The amount of power increases linearly with $\Psi$ due 
to the increase in the number of estimated links. Around $3 \%$ of signal processing power is spent on uplink and downlink MIMO processing [165]. Assuming an MMSE filter operation, the total MIMO processing within each CoMP cooperation cluster requires $\Psi^{3}$ operations [165]. Therefore, the average MIMO processing per base station only increases quadratically with $\Psi$, and the energy consumption due to the signal processing for each base station can be calculated as follows [165]:

$$
P_{s p}=p_{s p}\left(0.87+0.1|\Psi|+0.03|\Psi|^{2}\right)
$$

where $p_{s p}$ denotes a base line signal processing power per base station.

Backhaul is modeled as a collection of wireless micro wave links, matching the state-of-the-art in most cellular networks. Each link has a capacity of $100 \mathrm{Mbit}$ per second and spends $50 \mathrm{~W}$ power. Thus for a given average backhaul requirement $c_{b h}$ per base station, the backhaul power can be calculated [165]:

$$
P_{b h}=\frac{50 c_{b h}}{10^{8}}
$$

Substituting (5.5), (5.6) and (5.7) into (5.4), we can obtain:

$$
P_{B S}=\frac{a P_{\min }\left(\frac{D}{2}\right)^{\phi}}{K}+b p_{s p}\left(0.87+0.1|\Psi|+0.03|\Psi|^{2}\right)+\frac{50 c c_{b h}}{10^{8}}
$$

Namely,

$$
P_{B S}=U+V|\Psi|+W|\Psi|^{2}
$$

with $U=\frac{a P_{\min }\left(\frac{D}{2}\right)^{\phi}}{K}+0.87 b p_{s p}+\frac{50 c c_{b h}}{10^{8}}, V=0.1 b p_{s p}$, and $W=0.03 b p_{s p}$ 


\subsubsection{Problem Formulation}

Each retailer in smart grids provides the real-time price to the base stations in each cluster, and then each cluster will decide how many base stations need to be turned on, and how much electricity needs to be procured from each retailer in order to provide enough electricity for all of the active base stations in the cluster. The system is illustrated in Fig. 5.1. Each retailer and each cluster of base stations make decisions to maximize respective utility.

We employ a Stackelberg game-theoretical scheme to jointly consider the utility of the retailers and base stations in the cluster. Our proposed Stackelberg game can be considered a generalized Stackelberg game in which there are multiple leaders and one or more followers. According to the behaviors of the base stations in each cluster and those of the retailers, the proposed Stackelberg game is divided into two levels: a cellular network level game and a smart grid level game. The base stations in one cluster, as a Stackelberg follower, play a cellular network level game. On the other hand, each retailer, as a Stackelberg leader, plays a smart grid level game. The prices and the information about how much electricity to procure need to be exchanged between the retailers and the base stations in the cluster. The proposed two-level game-theoretical approach can be implemented in a distributed manner.

\subsubsection{Cellular Network Level Game}

The base stations in a cluster aim to obtain the lowest service blocking probability with the least possible costs. There is a trade-off between the blocking probability and the costs for the base stations. Therefore, it is very important for the base stations to decide how many base stations will be active in a cluster, and how much electricity is procured from each retailer in order to maximize utility.

With the increasing concerns about environmental protection, stricter regulations 
on pollutant emissions have been introduced, often including financial penalties associated with emissions (e.g., carbon credits), as well as non-financial costs associated with environmental damage, which is represented as financial penalties as well in our model. The appropriately designed pollutant emission costs make it possible for the base stations to procure electricity from retailers with renewable energy, thus reducing $\mathrm{CO}_{2}$ emissions. We assume this cost is passed to the electricity users.

Therefore, the net utility function of all base stations in the cluster can be defined as:

$$
\mathcal{U}_{s}=\xi \mathcal{F}\left(\mathcal{P}_{b}\right)-\sum_{n \in \mathcal{N}} p_{n} q_{n}-\sum_{n \in \mathcal{N}} \mathcal{I}_{n}\left(q_{n}\right)
$$

where $\xi$ is a weight, $\mathcal{F}\left(\mathcal{P}_{b}\right)$ is the utility function of the base stations with regards to the service blocking probability $\mathcal{P}_{b}, \mathcal{N}(\mathcal{N}=\{1, \ldots, N\})$ denotes the retailer set, $p_{n}$ denotes the price provided by Retailer $n, q_{n}$ denotes the amount of electricity procured from Retailer $n$ and the function $\mathcal{I}_{n}()$ denotes the total pollutant emission cost incurred by the base stations when they procure electricity from Retailer $n$.

The utility of the base station with regards to the service blocking probability is very high as long as the blocking probability is below a threshold value, and very low when the blocking probability passes the threshold. Therefore, the utility function $\mathcal{F}\left(\mathcal{P}_{b}\right)$ can be calculated as follows:

$$
\mathcal{F}\left(\mathcal{P}_{b}\right)=U_{\text {const }}-\rho \exp \left(\left(\mathcal{P}_{b}-\delta P_{t h}+G_{b k}\right) K_{b k}\right)
$$

where $P_{t h}$ denotes the blocking probability threshold value, $U_{c o n s t}, \rho, \delta, G_{b k}$ and $K_{b k}$ are parameters for defining the utility functions for users, which might change with people and time.

The emissions of a generating unit can be described by a quadratic function of the amount of electricity generated [166-168]. Therefore, we use the following function 
to model the pollutant emission cost for the base stations when procuring an amount of electricity $q_{n}$ from Retailer $n$ :

$$
\mathcal{I}_{n}\left(q_{n}\right)=\alpha_{n} q_{n}^{2}+\beta_{n} q_{n}
$$

where $\alpha_{n}>0, \beta_{n}>0$, and $\alpha_{n}$ and $\beta_{n}$ depends on the pollutant level of Retailer $n$.

Therefore, the optimization problem for the base stations in the cluster can be formulated as:

$$
\begin{aligned}
\max _{\Psi, q_{n}} \mathcal{U}_{s}= & \xi\left(U_{\text {const }}-\rho \exp \left(\left(\mathcal{P}_{b}-\delta P_{t h}+G_{b k}\right) K_{b k}\right)\right)-\sum_{n \in \mathcal{N}} p_{n} q_{n} \\
& -\sum_{n \in \mathcal{N}}\left(\alpha_{n} q_{n}^{2}+\beta_{n} q_{n}\right)
\end{aligned}
$$

s.t.

$$
\sum_{n \in \mathcal{N}} q_{n}=U|\Psi|+V|\Psi|^{2}+W|\Psi|^{3} .
$$

\subsubsection{Smart Grid Level Game}

We assume that each retailer is independent and acts selfishly, and aims to gain as much extra profit as possible. Retailer $n$ 's utility function can be defined as:

$$
\mathcal{U}_{n}=\left(p_{n}-c_{n}\right) q_{n}
$$

where $c_{n}$ denotes the cost of electricity for Retailer $n$. The optimization problem for Retailer $n$ is

$$
\max _{p_{n}} \mathcal{U}_{n}=\left(p_{n}-c_{n}\right) q_{n}, \forall n .
$$

The choice of the optimal price for each retailer is affected by the other retailers' prices and its own pollutant level, since the retailers compete to get selected by the base stations. If a certain retailer asks such a high price that makes it less beneficial 
than the other retailers to the base stations, then the base stations will procure less from that retailer. On the other hand, if the provided price is too low, the profit of that retailer will be unnecessarily low.

\subsubsection{Analysis of the Proposed Two-Level Game}

For a Stackelberg game, existence and uniqueness of a Stackelberg equilibrium are two desirable properties. If we know there exists exactly one equilibrium, we can predict the equilibrium strategy of the players and resulting performance of the system $[16,169]$. In this section, we first obtain the close-form solutions to the proposed game. Then, we prove that the solutions are the Stackelberg equilibrium for the proposed game. Finally, we prove that the Stackelberg equilibrium of the proposed game is unique.

\subsubsection{Analysis of the Cellular Network Level Game}

For an arbitrary combination set $\Psi$, we can represent the Lagrangian for (5.13) as follows:

$$
L_{b s}\left(q_{n}, \nu\right)=\mathcal{U}_{s}+\nu\left(\sum_{n \in \mathcal{N}} q_{n}-U|\Psi|-V|\Psi|^{2}-W|\Psi|^{3}\right)
$$

where $\nu$ is the Lagrangian multiplier. Set the derivative $d L_{b s}=0$, which yields the system of equations:

$$
\begin{aligned}
\frac{\partial L_{b s}}{\partial q_{1}} & =-p_{1}-2 \alpha_{1} q_{1}-\beta_{1}+\nu=0, \\
\vdots & \\
\frac{\partial L_{b s}}{\partial q_{N}} & =-p_{N}-2 \alpha_{N} q_{N}-\beta_{N}+\nu=0, \\
\frac{\partial L_{b s}}{\partial \nu} & =\sum_{n \in \mathcal{N}} q_{n}-U|\Psi|-V|\Psi|^{2}-W|\Psi|^{3}=0 .
\end{aligned}
$$


Therefore, for set $\Psi$, the optimal quantity of electricity $q_{n, \Psi}^{*}$ procured from Retailer $n$ can be obtained as follows:

$$
\begin{aligned}
q_{n, \Psi}^{*}= & \frac{1-2 \alpha_{n} X}{4 \alpha_{n}^{2} X} p_{n}+\sum_{j \neq n} \frac{1}{4 \alpha_{n} \alpha_{j} X} p_{j}+\frac{\left(1-2 \alpha_{n} X\right) \beta_{n}}{4 \alpha_{n}^{2} X}+\sum_{j \neq n} \frac{\beta_{j}}{4 \alpha_{n} \alpha_{j} X} \\
& +\frac{U|\Psi|+V|\Psi|^{2}+W|\Psi|^{3}}{2 \alpha_{n} X},
\end{aligned}
$$

where $X=\sum_{n \in \mathcal{N}} \frac{1}{2 \alpha_{n}}$, and $n, j \in \mathcal{N}$. Among all elements of the coordination set $\Theta$, the optimal combination set $\Psi^{*}$ is an element that maximizes the net utility of the base stations in the cluster. For set $\Psi^{*}$, the optimal quantity of electricity $q_{n, \Psi^{*}}^{*}$ procured from Retailer $n$ can be calculated as follows:

$$
\begin{aligned}
q_{n, \Psi^{*}}^{*}= & \frac{1-2 \alpha_{n} X}{4 \alpha_{n}^{2} X} p_{n}+\sum_{j \neq n} \frac{1}{4 \alpha_{n} \alpha_{j} X} p_{j}+\frac{\left(1-2 \alpha_{n} X\right) \beta_{n}}{4 \alpha_{n}^{2} X}+\sum_{j \neq n} \frac{\beta_{j}}{4 \alpha_{n} \alpha_{j} X} \\
& +\frac{U\left|\Psi^{*}\right|+V\left|\Psi^{*}\right|^{2}+W\left|\Psi^{*}\right|^{3}}{2 \alpha_{n} X}
\end{aligned}
$$

Property 1 The optimal amount of electricity $q_{n, \Psi^{*}}^{*}$ procured from Retailer $n$ decreases with its price $p_{n}$, when other retailers' prices are fixed.

Proof 5 Taking the first-order derivative of $q_{n, \Psi^{*}}^{*}$, we have

$$
\frac{\partial q_{n, \Psi^{*}}^{*}}{\partial p_{n}}=\frac{1-2 \alpha_{n} X}{4 \alpha_{n}^{2} X}
$$

Since $X>0$, and $1-2 \alpha_{n} X<0, \frac{\partial q_{n, \Psi^{*}}^{*}}{\partial p_{n}}$ is less than 0. Therefore, $q_{n, \Psi^{*}}^{*}$ is decreasing with $p_{n}$. 


\subsubsection{Analysis of the Smart Grid Level Game}

Substituting (5.21) into (5.15), we have

$$
\max _{p_{n}} \mathcal{U}_{n}=\left(p_{n}-c_{n}\right) q_{n, \Psi^{*}}^{*}
$$

Taking the derivative of $\mathcal{U}_{n}$ to $p_{n}$ and equating it to zero, we have

$$
\frac{\partial \mathcal{U}_{n}}{\partial p_{n}}=q_{n, \Psi^{*}}^{*}+\left(p_{n}-c_{n}\right) \frac{\partial q_{n, \Psi^{*}}^{*}}{\partial p_{n}}=0
$$

Therefore, the prices can be denoted as follows:

$$
\begin{gathered}
p_{1}+\frac{\alpha_{1}}{2\left(1-2 \alpha_{1} X\right)}\left(\sum_{j=2}^{N} \frac{p_{j}}{\alpha_{j}}\right)+\frac{\beta_{1}-c_{1}}{2} \\
+\frac{\alpha_{1}}{2\left(1-2 \alpha_{1} X\right)}\left(\sum_{j=2}^{N} \frac{\beta_{j}}{\alpha_{j}}\right)+\frac{\alpha_{1}}{1-2 \alpha_{1} X}\left(U\left|\Psi^{*}\right|+V\left|\Psi^{*}\right|^{2}+W\left|\Psi^{*}\right|^{3}\right)=0 \\
\frac{\alpha_{N}}{2\left(1-2 \alpha_{N} X\right)}\left(\sum_{j=1}^{N-1} \frac{p_{j}}{\alpha_{j}}\right)+p_{N}+\frac{\alpha_{N}}{2\left(1-2 \alpha_{N} X\right)}\left(\sum_{j=1}^{N-1} \frac{\beta_{j}}{\alpha_{j}}\right) \\
+\frac{\beta_{N}-c_{N}}{2}+\frac{\alpha_{N}}{1-2 \alpha_{N} X}\left(U\left|\Psi^{*}\right|+V\left|\Psi^{*}\right|^{2}+W\left|\Psi^{*}\right|^{3}\right)=0
\end{gathered}
$$

Solving the above equations of $p_{n}$, we denote the solutions as $p_{n}^{*}$.

Property 2 The utility function $\mathcal{U}_{n}$ of Retailer $n$ is concave in its own price $p_{n}$, when the other retailers' prices are fixed and its optimal amount of electricity provided can be calculated in (5.21). 
Proof 6 Taking the derivatives of $\mathcal{U}_{n}$ to $p_{n}$ results in

$$
\begin{aligned}
\frac{\partial \mathcal{U}_{n}}{\partial p_{n}}= & q_{n, \Psi^{*}}^{*}+\left(p_{n}-c_{n}\right) \frac{\partial q_{n, \Psi^{*}}^{*}}{\partial p_{n}} \\
= & \frac{1-2 \alpha_{n} X}{2 \alpha_{n}^{2} X} p_{n}+\sum_{j \neq n} \frac{1}{4 \alpha_{n} \alpha_{j} X} p_{j}+\frac{\left(1-2 \alpha_{n} X\right)\left(\beta_{n}-c_{n}\right)}{4 \alpha_{n}^{2} X}+\sum_{j \neq n} \frac{\beta_{j}}{4 \alpha_{n} \alpha_{j} X} \\
& +\frac{U\left|\Psi^{*}\right|+V\left|\Psi^{*}\right|^{2}+W\left|\Psi^{*}\right|^{3}}{2 \alpha_{n} X}
\end{aligned}
$$

and,

$$
\frac{\partial^{2} \mathcal{U}_{n}}{\partial p_{n}{ }^{2}}=\frac{1-2 \alpha_{n} X}{2 \alpha_{n}^{2} X}
$$

Since $1-2 \alpha_{n} X<0$ and $X>0$, we have $\frac{\partial^{2} \mathcal{U}_{n}}{\partial p_{n}{ }^{2}}<0$. Therefore, $\mathcal{U}_{n}$ is concave with respect to $p_{n}$.

\subsubsection{Existence of Stackelberg Equilibrium for the Proposed Two-Level Game}

In this subsection, we will prove that the solutions $q_{n, \Psi^{*}}^{*}$ and $p_{n}^{*}(n \in \mathcal{N})$ are the Stackelberg equilibrium for the proposed game.

Definition 5.2.1 $q_{n}^{S E}$ and $p_{n}^{S E}$ are the Stackelberg Equilibirum of the proposed twolevel game if for every Retailer $n$, when $p_{n}$ is fixed

$$
\mathcal{U}_{s}\left(\left\{q_{n}^{S E}\right\}\right)=\sup _{\left\{q_{n}\right\}} \mathcal{U}_{s}\left(\left\{q_{n}\right\}\right)
$$

and when $q_{n}$ is fixed

$$
\mathcal{U}_{n}\left(p_{n}^{S E}\right)=\sup _{p_{n}} \mathcal{U}_{n}\left(p_{n}\right)
$$

In the following, we prove that the solution $q_{n, \Psi^{*}}^{*}$ in $(5.21)$ is the global optimum that maximizes the base stations' utility $\mathcal{U}_{s}$. Namely, we verify that $q_{n, \Psi^{*}}^{*}$ in $(5.21)$ meets 
Karush-Kuhn-Tucer (KKT) conditions. Based on (5.16), we get

$$
\nabla L_{b s}\left(q_{n, \Psi^{*}}^{*}\right)=-p_{n}-2 \alpha_{n} q_{n, \Psi^{*}}^{*}-\beta_{n}+\nu=0
$$

and

$$
\nabla^{2} L_{b s}\left(q_{n, \Psi^{*}}^{*}\right)=-2 \alpha_{n}<0
$$

Therefore, $q_{n, \Psi^{*}}^{*}$ in (5.21) is the global optimum that maximizes the base station's utility $\mathcal{U}_{s}[170] . q_{n, \Psi^{*}}^{*}$ satisfies (5.29) and is the Stackelberg equilibrium $q_{n}^{S E}$. Due to the concavity of $\mathcal{U}_{n}$, Retailer $n$ can always find its optimal price $p_{n}^{*}$. Together, we construct the following theorem:

Theorem 7 The pair of $q_{n, \Psi^{*}}^{*}$ and $\left\{p_{n}^{*}\right\}$ is the Stackelberg equilibrium for the proposed two-level game, where the Stackelberg equilibrium is defined in (5.29) and (5.30).

\subsubsection{Uniqueness of the Stackelberg Equilibrium for the Proposed Two- Level Game}

We can show that the Stackelberg equilibrium of the proposed game is unique. Namely, we can prove that smart grid level game has the unique Nash equilibrium. The Nash equilibrium gives the set of prices such that none of the retailers can increase its individual utility by choosing a different price given the prices offered by the other retailers.

Because the retailers are independent and rational, and have the goal of maximizing their profits, the best response functions can be defined and used to obtain the solution of the pricing problem in smart grid level game. When the price strategies $\mathbf{p}_{-n}$ offered by the retailers other than Retailer $n$ are given, the best response function 
$\mathcal{B}_{n}\left(\mathbf{p}_{-n}\right)$ of Retailer $n$ can be defined as follows:

$$
\mathcal{B}_{n}\left(\mathbf{p}_{-n}\right)=\arg \max _{p_{n}} \mathcal{U}_{n}\left(p_{n}, \mathbf{p}_{-n}\right)
$$

Therefore, substituting (5.21) into (5.24), the best response function $\mathcal{B}_{n}\left(\mathbf{p}_{-n}\right)$ of Retailer $n$ is as follows:

$$
\mathcal{B}_{n}\left(\mathbf{p}_{-n}\right)=\frac{\alpha_{n}}{2\left(2 \alpha_{n} X-1\right)}\left(\sum_{j \neq n} \frac{p_{j}}{\alpha_{j}}\right)+Y_{n}
$$

where

$$
\begin{aligned}
Y_{n}= & \frac{\alpha_{n}}{2\left(2 \alpha_{n} X-1\right)}\left(\sum_{j \neq n} \frac{\beta_{j}}{\alpha_{j}}\right)+\frac{c_{n}-\beta_{n}}{2} \\
& +\frac{\alpha_{n}}{2 \alpha_{n} X-1}\left(U\left|\Psi^{*}\right|+V\left|\Psi^{*}\right|^{2}+W\left|\Psi^{*}\right|^{3}\right) .
\end{aligned}
$$

In the following, we will show that an arbitrary Retailer $n$ 's best response function $\mathcal{B}_{n}\left(\mathbf{p}_{-n}\right)$ is a standard function.

Definition 5.2.2 A function $\mathcal{B}_{n}\left(\boldsymbol{p}_{-n}\right)$ is standard if for all $\boldsymbol{p}_{-n} \geq 0$, the following properties are satisfied [171]:

- Positivity: $\mathcal{B}_{n}\left(\boldsymbol{p}_{-n}\right)>0$.

- Monotonicity: If $\boldsymbol{p}_{-n} \geq \boldsymbol{p}_{-n}^{\prime}$, then $\mathcal{B}_{n}\left(\boldsymbol{p}_{-n}\right) \geq \mathcal{B}_{n}\left(\boldsymbol{p}_{-n}^{\prime}\right)$.

- Scalability: For all $\omega>1, \omega \mathcal{B}_{n}\left(\boldsymbol{p}_{-n}\right)>\mathcal{B}_{n}\left(\omega \boldsymbol{p}_{-n}\right)$.

Proposition 5.2.1 An arbitrary Retailer $n$ 's best response function $\mathcal{B}_{n}\left(\boldsymbol{p}_{-n}\right)$ is standard.

1) Positivity. 
In (5.34),

$$
\mathcal{B}_{n}\left(\mathbf{p}_{-n}\right)=\frac{\alpha_{n}}{2\left(2 \alpha_{n} X-1\right)}\left(\sum_{j \neq n} \frac{p_{j}}{\alpha_{j}}\right)+Y_{n}
$$

Since $\alpha_{n}>0,2 \alpha_{n} X-1>0, \alpha_{j}>0$ and $p_{j}>0$, the first item of the RHS of the above equation is greater than 0 . Since $Y_{n}>0$, the best response function $\mathcal{B}_{n}\left(\mathbf{p}_{-n}\right)$ is positive.

2) Monotonicity.

Suppose $\mathbf{p}_{-n}$ and $\mathbf{p}_{-n}^{\prime}$ are different price vectors, and the vector inequlity $\mathbf{p}_{-n} \geq$ $\mathbf{p}_{-n}^{\prime}$ means that $p_{j} \geq p_{j}^{\prime}, \forall j \in\{1, \ldots, N\}, j \neq n$. If $\mathcal{B}_{n}\left(\left[p_{1}, \ldots, p_{j}, \ldots, p_{N}\right]\right) \geq$ $\mathcal{B}_{n}\left(\left[p_{1}, \ldots, p_{j}^{\prime}, \ldots, p_{N}\right]\right)$, then monotonicity can be shown to hold. Therefore, the problem reduces to provide $\frac{\partial \mathcal{B}_{n}\left(\mathbf{p}_{-n}\right)}{\partial p_{j}} \geq 0$. Taking the derivitive of the best response function $\mathcal{B}_{n}\left(\mathbf{p}_{-n}\right)$ to $p_{j}$, we obtain

$$
\frac{\partial \mathcal{B}_{n}\left(\mathbf{p}_{-n}\right)}{\partial p_{j}}=\frac{\alpha_{n}}{2 \alpha_{j}\left(2 \alpha_{n} X-1\right)}>0
$$

3) Scalability.

Based on (5.34), we can obtain

$$
\begin{aligned}
& \omega \mathcal{B}_{n}\left(\mathbf{p}_{-n}\right)=\frac{\alpha_{n} \omega}{2\left(2 \alpha_{n} X-1\right)}\left(\sum_{j \neq n} \frac{p_{j}}{\alpha_{j}}\right)+\omega Y_{n} . \\
& \mathcal{B}_{n}\left(\omega \mathbf{p}_{-n}\right)=\frac{\alpha_{n} \omega}{2\left(2 \alpha_{n} X-1\right)}\left(\sum_{j \neq n} \frac{p_{j}}{\alpha_{j}}\right)+Y_{n} .
\end{aligned}
$$

Based on (5.37) and (5.38), we can obtain

$$
\omega \mathcal{B}_{n}\left(\mathbf{p}_{-n}\right)-\mathcal{B}_{n}\left(\omega \mathbf{p}_{-n}\right)=(\omega-1) Y_{n}>0
$$

Therefore, for all $\omega>1, \omega \mathcal{B}_{n}\left(\mathbf{p}_{-n}\right)>\mathcal{B}_{n}\left(\omega \mathbf{p}_{-n}\right)$. 
Theorem 8 The pair of $q_{n, \psi^{*}}^{*}$ and $\left\{p_{n}^{*}\right\}$ is the unique Stackelberg equilibrium for the proposed two-level game, where the Stackelberg equilibrium is defined in (5.29) and (5.30).

Proof 7 Since an arbitrary Retailer n's best response function $\mathcal{B}_{n}\left(\boldsymbol{p}_{-n}\right)$ is standard, smart grid level game has the unique Nash equilibrium [172]. Therefore, the proposed game has the unique Stackelberg equilibrium.

\subsubsection{Model for Multiple Base Station Clusters}

In this subsection, we consider the extension of the proposed game to the situation where $S$ independent clusters of base stations are served by several retailers, where $s \in \mathcal{S}, S=|\mathcal{S}|$. We assume that each retailer in smart grids asks a uniform price no matter which cluster of base stations they supply with electricity. Therefore, Retailer $n$ 's utility function will be modified, following from (5.14):

$$
\mathcal{U}_{n}=\left(p_{n}-c_{n}\right)\left(\sum_{s \in \mathcal{S}} q_{n}\right)
$$

where $\sum_{s \in \mathcal{S}} q_{n}$ denotes the total electricity procured by these clusters of base stations from Retailer $n$.

When there are multiple clusters of base stations in the network, the proposed scheme is still applicable: the cellular-network level game for each cluster of base stations is essentially the same as the single cluster case. The only change is in smart grid level game, where the corresponding best response function can be calculated based on the following function instead of (5.24):

$$
\sum_{s \in \mathcal{S}} q_{n, \Psi^{*}}^{*}+\left(p_{n}-c_{n}\right)\left(\sum_{s \in \mathcal{S}} \frac{\partial q_{n, \Psi^{*}}^{*}}{\partial p_{i}}\right)=0
$$


The previous methods of analysis still apply in this situation.

\subsubsection{Simulation Results and Discussions}

In this section, we use computer simulations to evaluate the performance of the proposed dynamic base station operation scheme (DBSOS). In DBSOS, according to Subsection 5.2.3.3, we derive the optimal solution for the cellular network to decide how many base stations will be active in a cluster, and how much electricity is procured from each retailer in order to maximize utility. We also derive the optimal solution for retailers to decide the electricity price. In the simulations, we assume that there are three base stations in a cluster. These base stations can procure electricity from two retailers. We set the parameters as follows: $\mathrm{a}=7.35, \mathrm{~b}=2.9, \mathrm{c}=1$, $p_{s p}=58 \mathrm{~W}, 10 \log \left(P_{\min }\right)=-120, K=0.0001, \phi=4.0, D=800 \mathrm{~m}, c_{b h}=10^{9}$ bps [165]. We set $\lambda$ and $\mu$ as 1.1 and $1 / 180$, respectively. We choose $P_{t h}=0.1[173,174], c_{1}$ $=0.3$ cents $/ k W h, c_{2}=0.4$ cents $/ k W h, \beta=0.1$ cents $/ k W h, \alpha_{1}=2 \times 10^{-2}$ and $\alpha_{2}=5 \times 10^{-2}$. Parameter $\lambda$ is varied in Fig. 5.4, and $\alpha_{1}$ is varied in Fig. 5.7, Fig. 5.8 and Fig. 5.10. We compare the proposed DBSOS with the traditional method, which does not consider the dynamics of smart grid and all of the base stations are active, in terms of operational expenditure and $\mathrm{CO}_{2}$ emissions.

We first investigate how different coordinations among active base stations in DBSOS with CoMP affect the service blocking probability in the cluster and the total amount of electricity consumed. Fig. 5.3 shows that the service blocking probability starts at 1.0 when there are no active base stations in the cluster, gradually decreases, and finally reaches to 0.0 when three active base stations coordinate. Each base station consumes approximately $5 \times 10^{4} \mathrm{~W}$ electricity when it is active. The figure also shows that the amount of electricity consumed increases with the number of active base stations in the cluster. In this scenario, if one base station in the cluster 

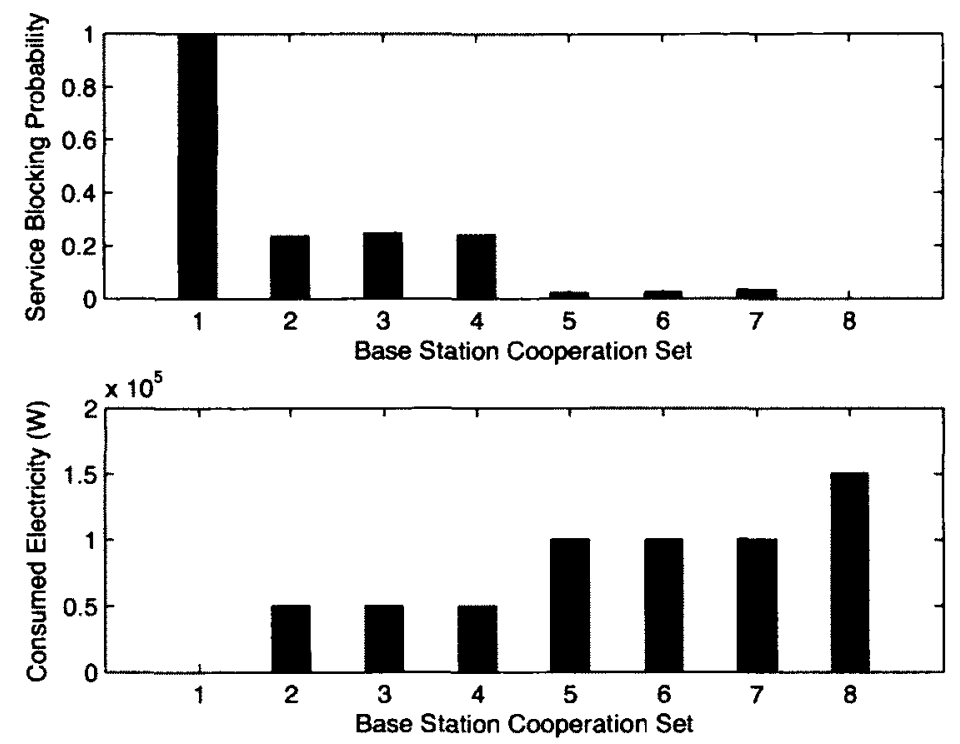

Figure 5.3: Service blocking probability and amount of electricity consumed as the combination of the base stations in a cluster varies, $\lambda=1.1$ and $\mu=1 / 180$. The numbers on the $x$ axis represent the indices of different elements of the cooperation set $\Theta$. In base station cooperation sets $2,3,4$, there is one active base station. In cooperation sets 5,6 and 7 , there are two active base stations. In cooperation set 8 , all three base stations are active.

is shut down, the service blocking probability is still less than the threshold 0.1 . The two active base stations in the cluster can extend their coverage using CoMP transmissions and receptions.

Fig. 5.4 shows that using the traditional method, these three base stations spend a constant amount of money to procure a constant amount of electricity, and therefore produce constant amount of $\mathrm{CO}_{2}$, even though the traffic arrival rate $\lambda$ varies. The figure also shows that for DBSOS, the number of base stations that need to be activated depends on the traffic arrival rate. For example, when the traffic arrival rate is less than 0.8 , one active base station is enough to meet the service blocking probability requirement. $\mathrm{CO}_{2}$ emissions depend on how many base stations that are active during that period. DBSOS has a much better performance compared to the 

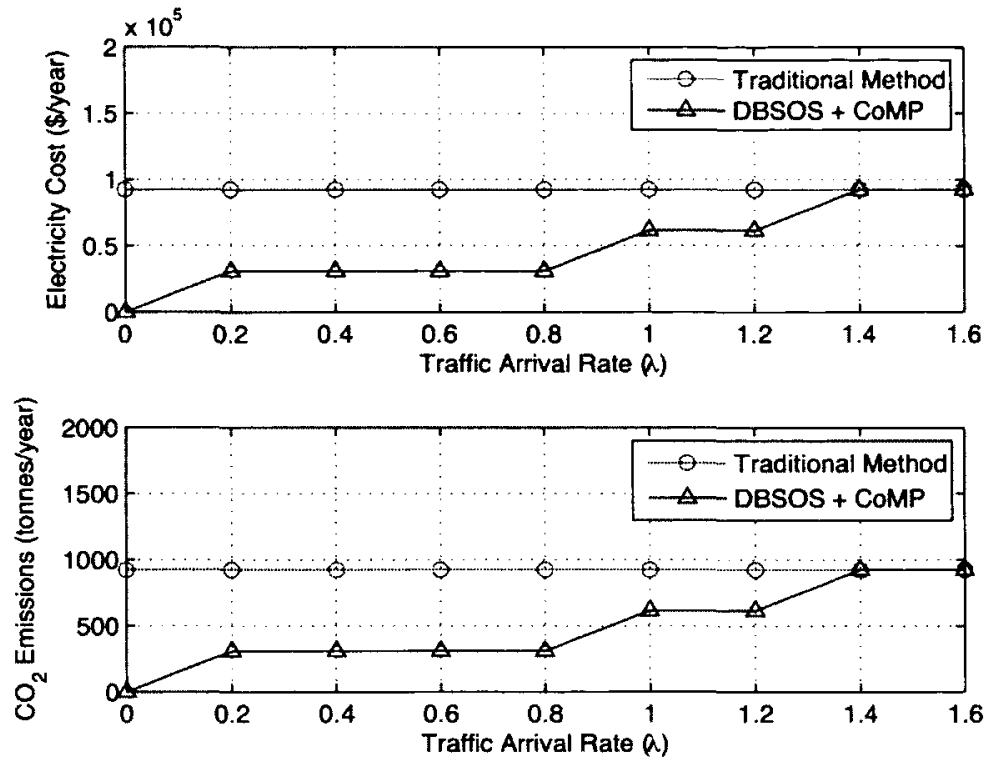

Figure 5.4: Compare the traditional method with the proposed DBSOS with CoMP, in terms of electricity cost and $\mathrm{CO}_{2}$ emission as the traffic arrival rate $\lambda$ varies.

traditional method in terms of electricity costs and $\mathrm{CO}_{2}$ emissions, especially when the traffic arrival rate is low. For example, DBSOS leads to a decrease $66.7 \%$ in electricity costs and $\mathrm{CO}_{2}$ emissions when the traffic arrival rate is between 0.2 and 0.8 compared to the traditional method.

We compare the traditional method, DBSOS with CoMP, and DBSOS with CoMP and demand response (i.e., in smart grids). This comparison is in terms of the operational expenditure of the base stations and the service blocking probability when negative electricity price is sometimes offered in smart grids. The electricity price is set to $0.0464 \$ / k W h$, the average negative electricity price is set to -0.2356 $\$ / k W h[12,14,15]$. Negative price is offered $5 \%$ of the time, and the value can increase with the higher amount of renewable energy integrated into smart grids. Fig. 5.5 shows that the traditional method has the highest operational expenditure. Compared to DBSOS with CoMP, DBSOS with CoMP in smart grids decreases the 


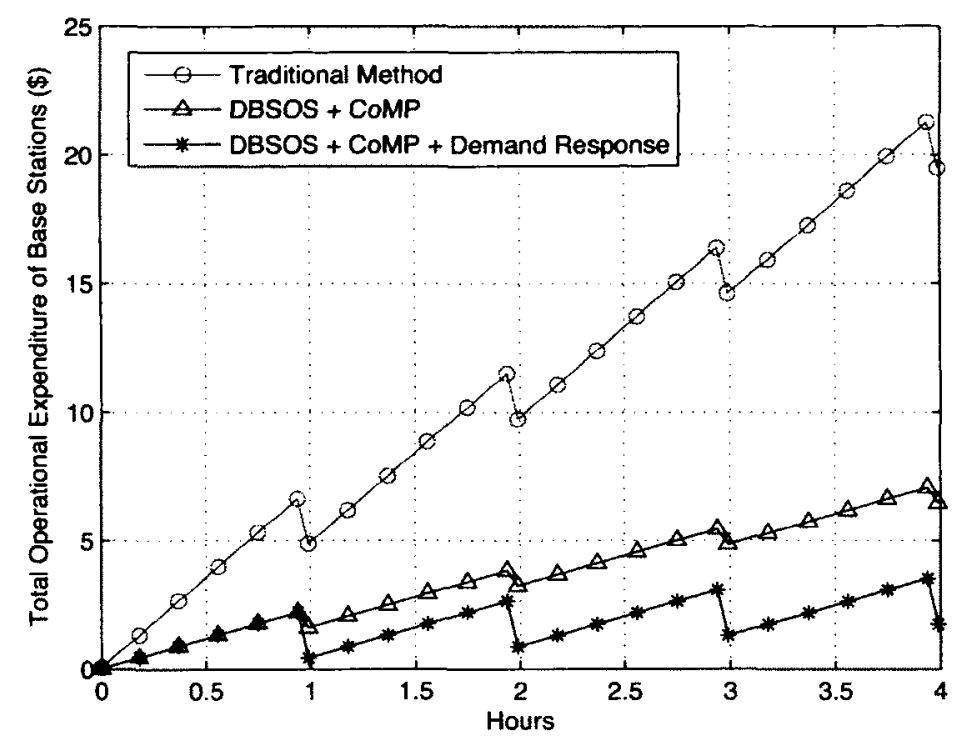

Figure 5.5: Total operational expenditure of base stations.

operational expenditure by $27.06 \%$, even though the negative price is offered only rarely. The reason for this is that in the BSSOS with CoMP in smart grids, the operation of the base stations depends not only on the service blocking probability, but also on the price of offered by smart grids. Therefore, all of the base stations might be turned on when the electricity price is low or negative.

We check how the utility of Retailer 1 and Retailer 2 is affected by the electricity price offered by Retailer 1 in the proposed game. Fig. 5.6 shows that utility function of Retailer 1 is concave in price $p_{1}$, and utility of Retailer 2 increases with price $p_{1}$. For each price offered by Retailer 1, Retailer 2 offers a price based on the best response strategy to maximize its own utility. The amount of electricity procured from Retailer 1 decreases with the increase of the price offered by Retailer 1, and the amount of electricity procured from Retailer 2 correspondingly increases.

We also study how the pollutant emission levels of Retailer 1 and Retailer 2 affect the optimal amount of electricity and the optimal prices at the Stackelberg equilibrium 


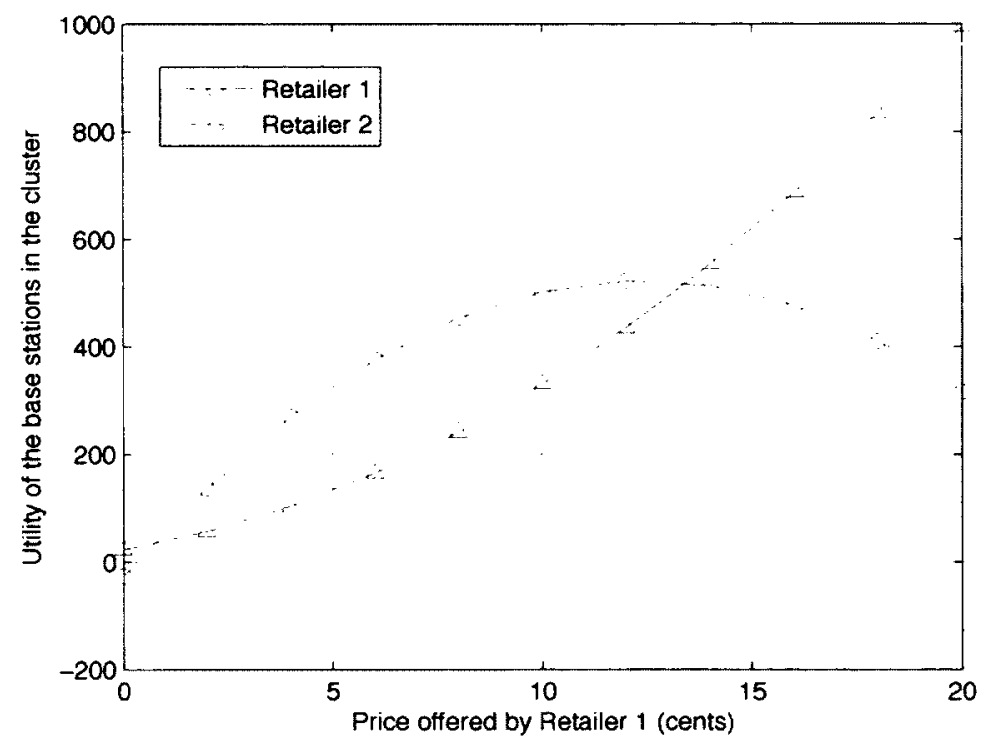

Figure 5.6: Utility of Retailer 1 and Retailer 2 as the price offered by Retailer 1 varies.

in the proposed two-level game. Fig. 5.7 shows that the optimal prices offered by Retailer 1 and Retailer 2 increase with the increase of Retailer 1's pollutant level. The retailer with a lower pollutant level can offer a higher price. Fig. 5.8 shows that the optimal amount of electricity procured from Retailer 1 decreases and that from Retailer 2 increases with the increase of Retailer 1's pollutant level, and the total amount of electricity procured from both retailers is constant. The figure also shows that higher amount of electricity is procured from the retailer with the lower pollutant level. The crossing situation in these two figures show that retailers offer the same optimal price and electricity is procured equally from these two retailers when they have the same pollutant level.

We investigate how the number of retailers affects the utility of the base stations in the cluster in the proposed game. Fig. 5.9 shows that the utility increases with the increase of the number of retailers and parameter $\xi$. As the number of retailers 


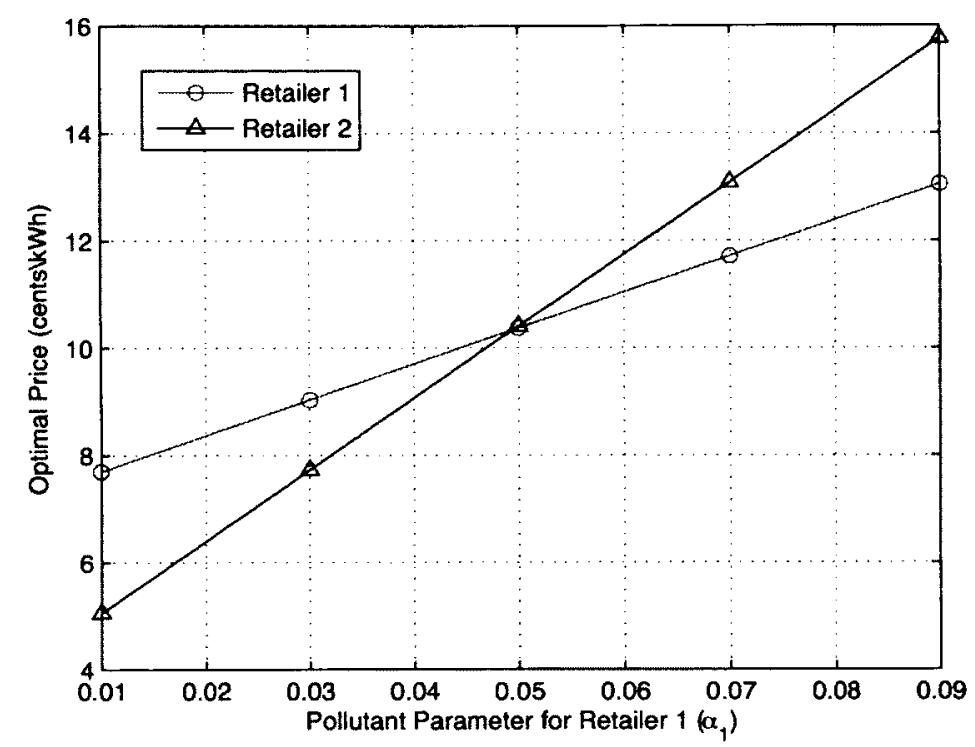

Figure 5.7: Optimal prices offered by Retailer 1 and Retailer 2 as the pollutant parameter of Retailer $1\left(\alpha_{1}\right)$ varies.

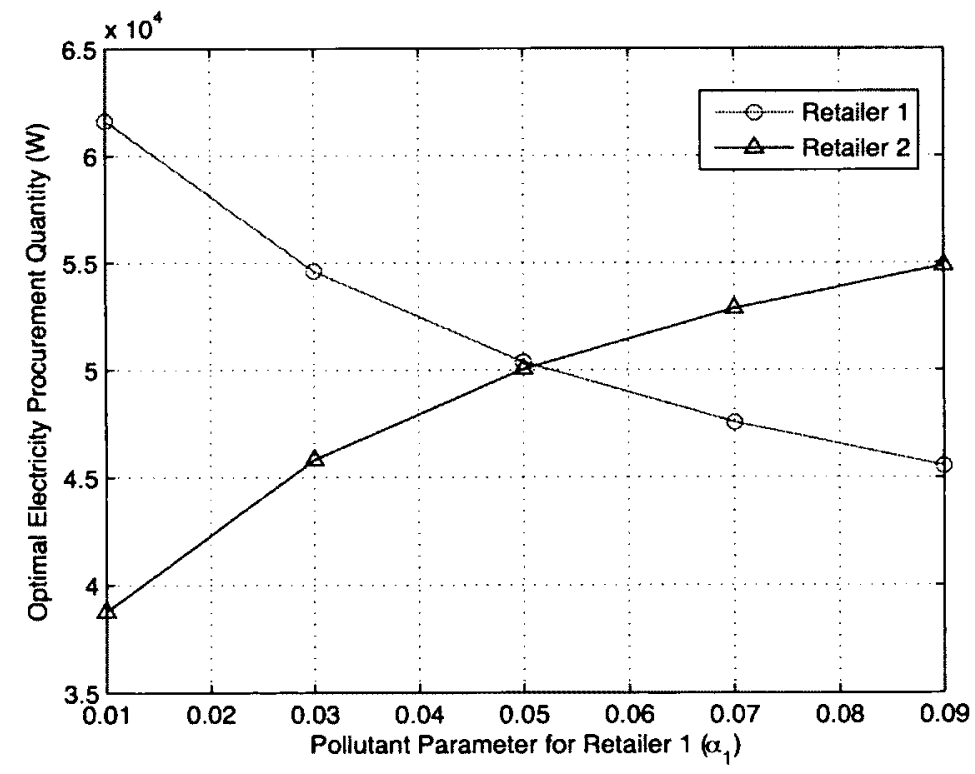

Figure 5.8: Optimal amount of electricity to procure from Retailer 1 and Retailer 2 as the pollutant parameter of Retailer $1\left(\alpha_{1}\right)$ varies. 


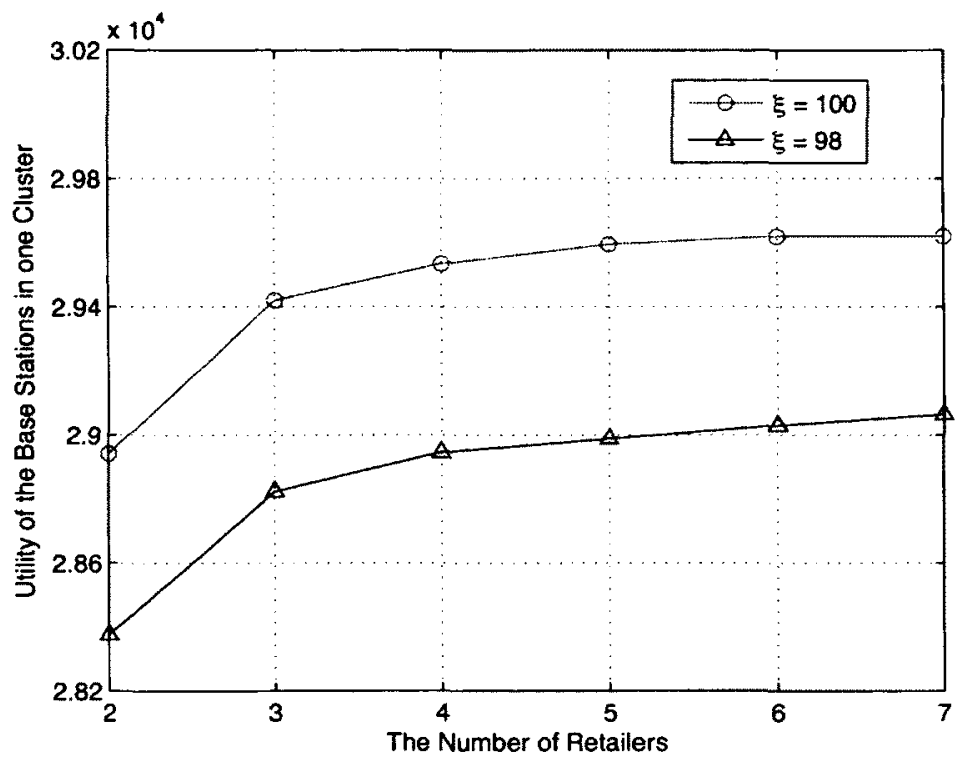

Figure 5.9: Utility of the optimal base station combination element $\Psi^{*}$ as the number of retailers varies.

increases, the competition among them becomes more severe, so the average price decreases. We finally compare the proposed scheme with or without considering the pollutant levels of retailers in terms of $\mathrm{CO}_{2}$ emissions. The scheme without considering the retailers' pollutant level means that the base stations only procure electricity from the retailer offering the lowest price. Fig. 5.10 shows that using the proposed scheme, $\mathrm{CO}_{2}$ emissions increase as the pollutant level of Retailer 1 increases. For the method without considering the pollutant levels, $\mathrm{CO}_{2}$ emissions are constant before the crossing point where two retailers have the same pollutant level, and linearly increase when Retailer 1 has a higher pollutant level than Retailer 2. This figure shows that our proposed scheme can further decrease the cost and $\mathrm{CO}_{2}$ emissions when the pollutant levels of retailers are considered. 


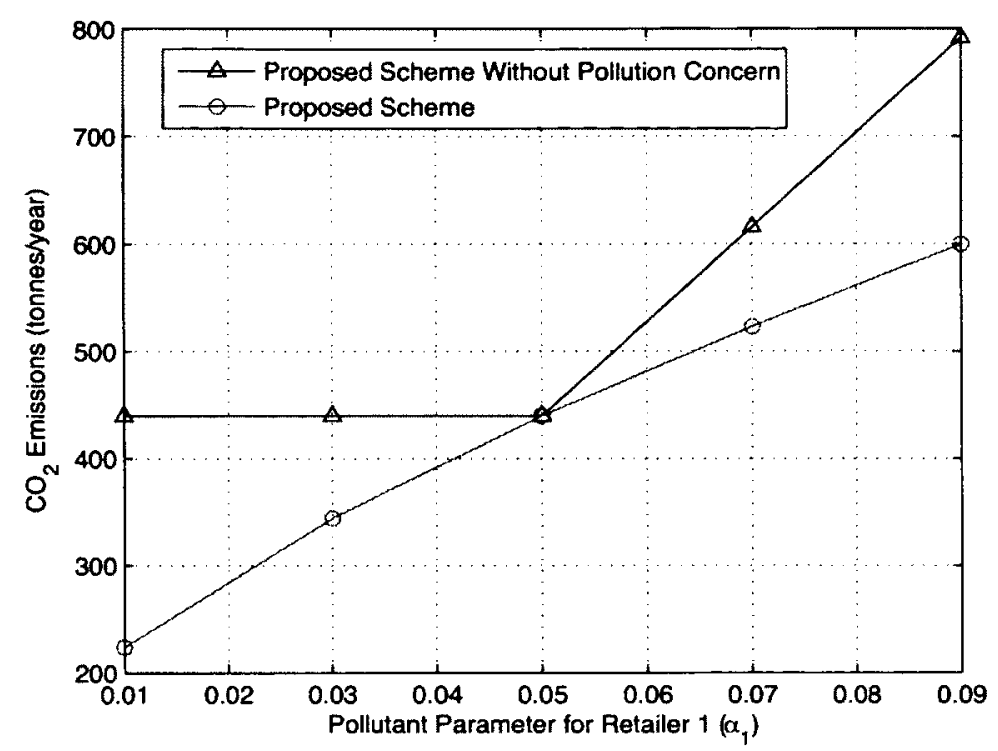

Figure 5.10: Compare these two schemes in terms of $\mathrm{CO}_{2}$ emissions as the pollutant parameter of Retailer $1\left(\alpha_{1}\right)$ varies.

\subsection{Dynamic Energy-Efficient Resource Alloca- tion in Cognitive Heterogeneous Wireless Net- works}

In this section, heterogeneous networks, cognitive radios and smart grids have been combined to improve the energy-efficiency of cellular networks. This section is organized as follows. We describe the system models in Section 5.3.1. The problem is formulated as a three-level Stackelberg game in Section 5.3.2. The proposed game is analyzed in Section 5.3.3. Simulation results are presented and discussed in Section 5.3 .4 . 


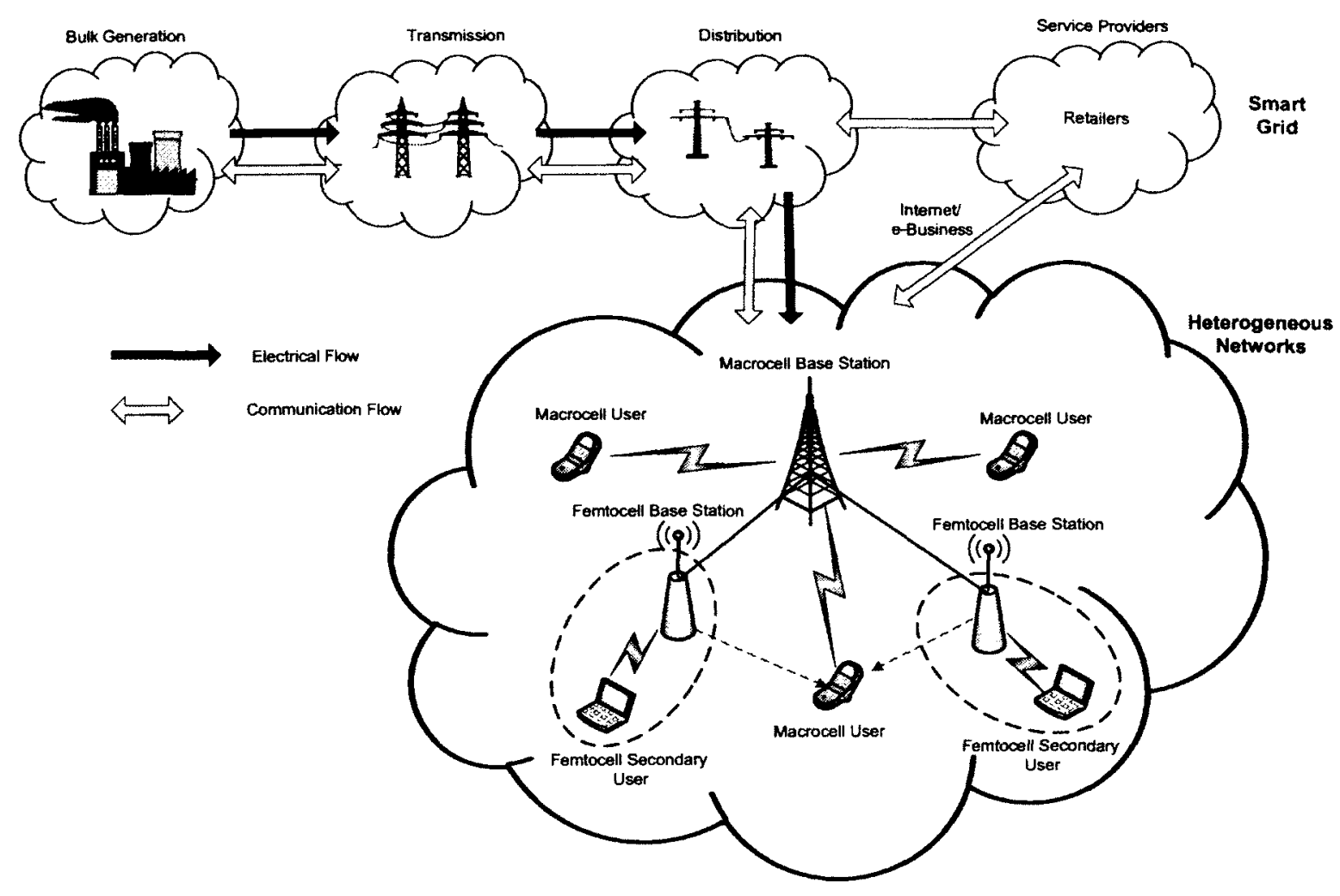

Figure 5.11: Heterogeneous networks powered by smart grids.

\subsubsection{System Models}

We consider a wireless heterogeneous network with cognitive radios and femtocells, which is powered by smart grids, as shown in Fig. 5.11. In this section, we first introduce our wireless heterogeneous network model. Then, we present an electricity consumption model for this heterogeneous network. Finally, we describe the complete system model.

\subsubsection{Wireless Heterogeneous Network Model}

We consider that in each service region there is one central MBS and multiple FBSs, each of which is connected to the MBS over a broadband connection, such as cable modem or digital subscriber line (DSL) [175]. In each femtocell, the FBS provides 
service for several users. We assume that the MBS is aware of spectrum access by the femtocells, and the FBSs can monitor the surrounding radio channel environment and are allowed the randomly access to the sub-bands. The system is operated in a time-slotted manner. In each time slot, the spectrum resource licensed to the MBS is divided into multiple subchannels. The macro users (MUs) use orthogonal frequencydivision multiple access (OFDMA) technology to access to the MBS. We assume there is one scheduled active user during each signaling slot in each femtocell, which is commonly assumed in the literature due to the opportunistic scheduling operation in practice [176].

We assume that MBS and femtocells share spectrum in the network, as is often the case in real networks [177]. Each sub-band of the MBS is allocated to one MU, and several nearby femtocells also use this sub-band. Since the femtocells share spectrum with the MBS, the cross-tier interference will greatly restrict the network performance. To mitigate the interference effect and guarantee the QoS, an interference price is proposed to allow the MBS to protect itself by charging the femtocell users. The femtocells will adaptively adjust the transmission power or change their access of sub-bands based on the channel condition and the interference price offered by the MBS. In the scenario we consider, the femtocells are deployed sparsely, so that the mutual interference between the femtocells is negligible. This is because the channel power gain drops sharply with the increase of the distance between femtocells due to path loss. Since femtocells are usually deployed indoors, the penetration loss is also significant. Therefore, it is reasonable to assume that the interference between femtocells can be ignored when the femtocells are sparsely deployed. 


\subsubsection{Electricity Consumption Model for Base Stations in Heteroge- neous Networks}

During the past decades, energy efficiency, which is commonly defined as information bits per unit transmit energy, has been studied from the information-theoretic perspective for various scenarios [178]. For energy-efficient communications, both transmit power and other parts of energy consumption need to be taken into consideration. Moreover, this additional energy consumption changes the fundamental trade-off between energy efficiency and data rate [179]. We consider that for energyefficient communications, it is desirable to maximize transmission rate and also take the electricity cost into account [101]. The energy-efficient metric used for each BS is its weighted transmission rate minus the weighted electricity cost. The electricity cost of the BS equals to the amount of consumed electricity times the real-time price offered by the chosen retailer in smart grids.

For each BS, total electricity consumption $P_{\text {total }}$ can be denoted as [180]

$$
P_{\text {total }}=\frac{P_{t}}{\eta}+N P_{D y n}+P_{S t a}
$$

where $P_{t}$ denotes the total transmit power, $\eta$ denotes the power amplifier (PA) efficiency, $N P_{D y n}$ denotes the dynamic power consumption, $N$ denotes the total BS antenna number, and $P_{S t a}$ denotes the static power, which is independent of transmit power, the number of active transmit antennas and bandwidth.

\subsubsection{Complete System Model}

We assume that there are $R$ electricity retailers in smart grids, one MBS and $K$ femtocells in the system. Each sub-band is allocated to one MU, and the nearby $K$ femtocells also use the same sub-band as MU. Therefore, there is interference between 
these $K$ femtocells and the MU. We only demonstrate the situation of one sub-band, since we mainly focus on how the decisions in smart grids, the MBS and FBSs affect each other. The model can be easily extended to multiple sub-bands situations.

The system performs as follows: each electricity retailer in smart grids offers a real-time electricity price to the MBS and FBSs. The MBS performs energy-efficient power allocation and procures electricity from the retailer offering the lowest price. The MBS also imposes a unified price on received interference from femtocell users in the network. Each femtocell updates its power allocation strategy, and also chooses the electricity retailer with the lowest offered price to procure electricity to maximize its net utility based on the interference price and the electricity price. We assume that each chosen retailer can provide enough electricity for the MBS and the FBSs.

\subsubsection{Problem Formulation}

In this section, we formulate the above problem as a three-stage Stackelberg game, jointly considering the utility maximization of the retailers in smart grids and the MBS and the FBSs in the heterogeneous network, as shown in Fig. 5.12. In stage I, each retailer in smart grids, as a leader in the Stackelberg game, offers a real-time electricity price $x_{r}$ to the MBS and also FBSs. In Stage II, the MBS, as a follower in Stage I, first decides which retailer it procure electricity from, and the amount of the electricity consumed $q_{m}$ (i.e., performs the energy-efficient power allocation $p_{m}$ ) based on the electricity prices offered by the retailers. Following this, the cognitive MBS acts as a leader in Stage II, and offers an interference price $y$ to the FBSs. In Stage III, each FBS decides on the retailer to procure electricity and the amount of the electricity it consumes by performing energy-efficient power allocation. 


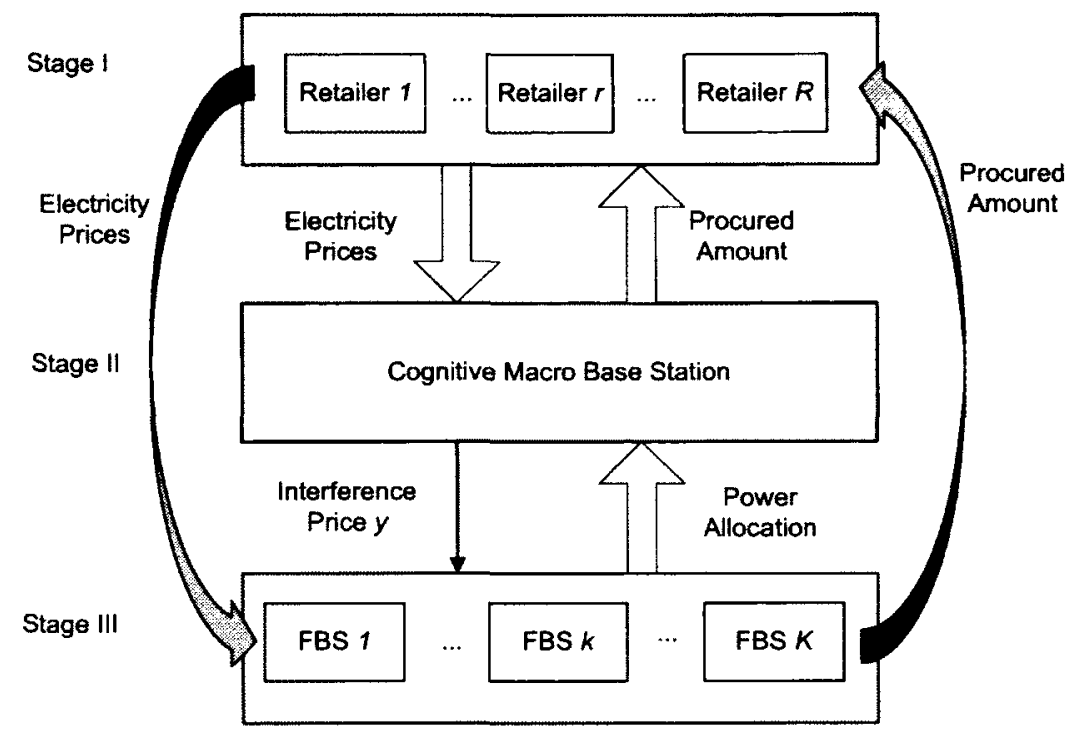

Figure 5.12: Three stage Stackelberg game.

\subsubsection{Smart Grid Level Game}

We assume that the electricity cost for each retailer includes the electricity procurement cost, and other costs, such as $\mathrm{CO}_{2}$ tax fees if the procured electricity is produced by non-renewable energy generators. We assume that the electricity cost for each electricity retailer is different from each other. Each retailer aims to gain as much profit as possible. Therefore, for an arbitrary Retailer $r$, its net utility function $\mathcal{U}_{r}$ can be defined as:

$$
\mathcal{U}_{r}=\left(x_{r}-c_{r}\right)\left(q_{m} s_{r m}+\sum_{k=1}^{K} q_{k} s_{r k}\right),
$$

where $x_{r}$ denotes the real-time electricity price, $c_{r}$ denotes the electricity cost, $q_{m}$ and $q_{k}$ denotes the amount of electricity needed by the MBS and by FBS $k$ respectively. $s_{r m}=1$ and $s_{r k}=1$ denote that MBS $m$ and FBS $k$ procure electricity from Retailer $r$ respectively. The optimization problem for Retailer $r$ can be defined as:

$$
\mathcal{U}_{r}^{*}=\max _{x_{r} \geq c_{r}}\left\{\left(x_{r}-c_{r}\right)\left(\left(P_{m f}+B_{m v} p_{m}\right) s_{r m}+\sum_{k=1}^{K}\left(P_{k f}+B_{k v} p_{k}\right) s_{r k}\right)\right\},
$$


where $P_{m f}$ and $P_{k f}$ denote the additional power consumed by MBS $m$ and FBS $k$ respectively, and $p_{m}$ and $p_{k}$ denote the transmit power consumed by MBS $m$ and femtocell BS $k$ respectively, and $B_{m v}$ and $B_{k v}$ denote 1 over the PA efficiency of MBS $m$ and FBS $k$ respectively.

\subsubsection{MBS Level Game}

For base stations, it is desirable to maximize the transmission rate with a given amount of energy cost. The MBS tries to achieve this by performing energy-efficient power allocation. The base station also needs to limit the interference from femtocells by offering the interference price. Therefore, the net utility function of the MBS can be calculated as:

$$
\begin{aligned}
\mathcal{U}_{m}= & W \log _{2}\left(1+\frac{p_{m} h_{m}^{2}}{\sigma_{m}^{2}+\sum_{k=1}^{K} g_{k m}^{2} p_{k}}\right)-\alpha\left(\sum_{r=1}^{R} x_{r}\left(P_{m f}+B_{m v} p_{m}\right) s_{r m}\right) \\
& +\beta y\left(\sum_{k=1}^{K} g_{k m}^{2} p_{k}\right)
\end{aligned}
$$

where $W$ denotes the transmission bandwidth of each subchannel, $p_{m}$ denotes the transmit power of the MBS on the subchannel, $h_{m}$ denotes the channel gain from MBS to MU $m$ including the path-loss and the smart scale fading process, $\sigma_{m}$ is the additive Gaussian white noise $(A G W N)$ with zero mean and unit variation, $g_{k m}$ denotes the channel gain between femtocell $k$ and MU $m$ including the path-loss and the small scale fading process, and $p_{k}$ denotes the transmit power for femtocell $k$, and $y$ denotes the interference price. $\beta$ and $\alpha$ denote a weight, which represents the trade-off between the transmission rate, energy cost and interference revenue. Equation (5.45) means that the MBS gains interference revenue from femtocells with the trade-off of decreasing the transmission rate. The optimization problem for the 
MBS can be formulated as:

$$
\begin{aligned}
\mathcal{U}_{m}^{*}= & \max _{s_{r m}, p_{m} \geq 0, y \geq 0}\left\{W \log _{2}\left(1+\frac{p_{m} h_{m}^{2}}{\sigma_{m}^{2}+\sum_{k=1}^{K} g_{k m}^{2} p_{k}}\right)\right. \\
& \left.-\alpha\left(\sum_{r=1}^{R} x_{r}\left(P_{m f}+B_{m v} p_{m}\right) s_{r m}\right)+\beta y\left(\sum_{k=1}^{K} g_{k m}^{2} p_{k}\right)\right\} .
\end{aligned}
$$

\subsubsection{FBS Level Game}

The FBSs need to choose which retailer they procure electricity from, and also can adaptively allocate transmission power to maximize its individual net utility based on the interference prices provided by the MBS and also electricity prices offered by the retailers in smart grids. Therefore, the net utility function for an arbitrary Femtocell $k$ can be defined as:

$$
\mathcal{U}_{k}=W \log _{2}\left(1+\frac{p_{k} h_{k}^{2}}{\sigma_{k}^{2}}\right)-\mu_{k}\left(\sum_{r=1}^{R} x_{r}\left(P_{k f}+B_{k v} p_{k}\right) s_{r k}\right)-\lambda_{k} y g_{k m}^{2} p_{k}
$$

where $h_{k}$ denotes the channel gain between Femtocell $k$ and FSU including the pathloss and the smart scale fading process, $\sigma_{k}$ denotes the effect of an interference from the MBS and the AGWN. $\mu_{k}$ and $\lambda_{k}$ denote a weight, which represent the trade-off between the transmission rate, energy cost and interference cost. The optimization problem for an arbitrary femtocell $k$ can be formulated as:

$$
\mathcal{U}_{k}^{*}=\max _{s_{r k}, p_{k} \geq 0}\left\{W \log _{2}\left(1+\frac{p_{k} h_{k}^{2}}{\sigma_{k}^{2}}\right)-\mu_{k}\left(\sum_{r=1}^{R} x_{r}\left(P_{k f}+B_{k v} p_{k}\right) s_{r k}\right)-\lambda_{k} y g_{k m}^{2} p_{k}\right\}
$$




\subsubsection{Analysis of the Proposed Three-Level Game}

In this section, we will analyze the proposed three-level game, and obtain the Stackelberg equilibrium of this game. Based on the above analysis, we know that each stage's strategy will affect other stages' strategies. Therefore, we use a backward induction method to analyze the proposed game.

\subsubsection{Analysis of Power Allocation Game for FBSs}

In order to maximize its net utility, each FBS will choose a proper retailer to procure electricity, and also adjust its individual power allocation strategy based on the interference price offered by the MBS and the electricity prices offered by the retailers in smart grids.

For an arbitrary FBS $k(k \in\{1, \ldots, K\})$, taking the derivative of $\mathcal{U}_{k}$ with respect to $p_{k}$ and equating it to zero, we have

$$
\frac{\partial \mathcal{U}_{k}}{\partial p_{k}}=\frac{W h_{k}^{2}}{\left(\sigma_{k}^{2}+p_{k} h_{k}^{2}\right) \ln 2}-\mu_{k}\left(\sum_{r=1}^{R} x_{r} B_{k v} s_{r k}\right)-\lambda_{k} y g_{k m}^{2}=0
$$

and

$$
\frac{\partial^{2} \mathcal{U}_{k}}{\partial p_{k}^{2}}=\frac{-W h_{k}^{4}}{\left(\sigma_{k}^{2}+p_{k} h_{k}^{2}\right)^{2} \ln 2}<0
$$

Therefore, the optimal power allocation strategy of an arbitrary FBS $k$ can be denoted as:

$$
p_{k}^{*}=\left[\frac{W}{\left(\mu_{k}\left(\sum_{r=1}^{R} x_{r} B_{k v} s_{r k}\right)+\lambda_{k} y g_{k m}^{2}\right) \ln 2}-\frac{\sigma_{k}^{2}}{h_{k}^{2}}\right]^{+}
$$

where

$$
[\cdot]^{+} \triangleq \max (\cdot, 0)
$$

For an arbitrary FBS $k$, the retailer offering lowest price will be chosen, which defines 
as $s_{r^{*} k}=1$. Therefore, the optimal power allocation stratgy of FBS $k$ is:

$$
p_{k}^{*}=\left[\frac{W}{\left(\mu_{k} x_{r_{k}^{*}} B_{k v}+\lambda_{k} y g_{k m}^{2}\right) \ln 2}-\frac{\sigma_{k}^{2}}{h_{k}^{2}}\right]^{+}
$$

\subsubsection{Analysis of the MBS Level Game}

In order to maximize its net utility, the MBS chooses a proper retailer to procure electricity from, and adjusts its individual power allocation strategy and offers an interference price to the FBSs based on the energy-efficient power allocation response of the FBSs. When the response strategy of each FBS is given based on the above subsection, substituting (5.53) into (5.45), we have

$$
\begin{aligned}
\mathcal{U}_{m}= & W \log _{2}\left(1+\frac{p_{m} h_{m}^{2}}{\sigma_{m}^{2}+\sum_{k=1}^{K} g_{k m}^{2}\left[\frac{W}{\left(\mu_{k} x_{r_{k}^{*}} B_{k v}+\lambda_{k} y g_{k m}^{2}\right) \ln 2}-\frac{\sigma_{k}^{2}}{h_{k}^{2}}\right]^{+}}\right) \\
& -\alpha \sum_{r=1}^{R} x_{r}\left(P_{m f}+B_{m v} p_{m}\right) s_{r m} \\
& +\beta y\left(\sum_{k=1}^{K} g_{k m}^{2}\left[\frac{W}{\left(\mu_{k} x_{r_{k}^{*}} B_{k v}+\lambda_{k} y g_{k m}^{2}\right) \ln 2}-\frac{\sigma_{k}^{2}}{h_{k}^{2}}\right]^{+}\right) .
\end{aligned}
$$

The net utility function $\mathcal{U}_{m}$ is a function of transmit power $p_{m}$ and interference price $y$. In order to maximize the net utility function $\mathcal{U}_{m}$, we can use the decomposition theory [181] and decompose the optimization problem into two sub optimization problems: fix interference price $y$ and allocate the power $p_{m}$ to maximize $\mathcal{U}_{m}$, and then find the optimal interference price.

We let

$$
I_{m}=\sigma_{m}^{2}+\sum_{k=1}^{K} g_{k m}^{2}\left[\frac{W}{\left(\mu_{k} x_{r_{k}^{*}} B_{k v}+\lambda_{k} y g_{k m}^{2}\right) \ln 2}-\frac{\sigma_{k}^{2}}{h_{k}^{2}}\right]^{+}
$$


Taking the derivative of $\mathcal{U}_{m}$ with respect to $p_{m}$ and equating it to zero, we have

$$
\frac{\partial \mathcal{U}_{m}}{\partial p_{m}}=\frac{W h_{m}^{2}}{\left(I_{m}+p_{m} h_{m}^{2}\right) \ln 2}-\alpha\left(\sum_{r=1}^{R} x_{r} B_{m v} s_{r m}\right)=0
$$

Therefore, the optimal power allocation strategy of MBS $m$ is as follows:

$$
p_{m}^{*}=\left[\frac{W}{\alpha\left(\sum_{r=1}^{R} x_{r} B_{m v} s_{r m}\right) \ln 2}-\frac{I_{m}}{h_{m}^{2}}\right]^{+} .
$$

For MBS $m$, the retailer with lowest electricity price will be chosen, which defines as $s_{r * m}=1$. Therefore, the $p_{m}^{*}$ can be calculated as:

$$
p_{m}^{*}=\left[\frac{W}{\alpha x_{r_{m}^{*}} B_{m v} \ln 2}-\frac{I_{m}}{h_{m}^{2}}\right]^{+}
$$

Due to the piecewise nature of price $y$, we introduce the indicator function for $k \in\{1, \ldots, K\}$

$$
V_{k}= \begin{cases}1, & y<\frac{\frac{W h_{k}^{2}}{\sigma_{k}^{2} \ln 2}-\mu_{k} x_{r_{k}^{*}} B_{k v}}{\lambda_{k} g_{k m}^{2}} \\ 0, & \text { otherwise. }\end{cases}
$$

Therefore, we can rewrite (5.54) as

$$
\mathcal{U}_{m}(y)=\mathcal{U}_{m 1}(y)+\mathcal{U}_{m 2}(y)
$$


where $\mathcal{U}_{m 1}(y)$ and $\mathcal{U}_{m 2}(y)$ can be described as follows:

$$
\begin{aligned}
\mathcal{U}_{m 1}(y)= & W \log _{2}\left(1+\frac{p_{m} h_{m}^{2}}{\sigma_{m}^{2}+\sum_{k=1}^{K} g_{k m}^{2} V_{k}\left(\frac{W}{\left(\mu_{k} x_{r_{k}^{*}} B_{k v}+\lambda_{k} y g_{k m}^{2}\right) \ln 2}-\frac{\sigma_{k}^{2}}{h_{k}^{2}}\right)}\right) \\
\mathcal{U}_{m 2}(y)= & \beta y\left(\sum_{k=1}^{K} g_{k m}^{2} V_{k}\left(\frac{W}{\left(\mu_{k} x_{r_{k}^{*}} B_{k v}+\lambda_{k} y g_{k m}^{2}\right) \ln 2}-\frac{\sigma_{k}^{2}}{h_{k}^{2}}\right)\right) \\
& -\alpha \sum_{r=1}^{R} x_{r}\left(P_{m f}+B_{m v} p_{m}\right) s_{r m} .
\end{aligned}
$$

Since $\mathcal{U}_{m}$ is a piecewise function of $y$, it can not get the derivation directly to solve $y$. However, when given $V_{k}$, function $\mathcal{U}_{m}$ is a continue differentiable function. We let $L_{k}(k \in\{1, \ldots, K\})$ as

$$
L_{k}=\frac{\frac{W h_{k}^{2}}{\sigma_{k}^{2} \ln 2}-\mu_{k} x_{r_{k}^{*}} B_{k v}}{\lambda_{k} g_{k m}^{2}} \text {. }
$$

We need to sort all $L_{k}$ in the order of ascending, and without loss generality, let that $L_{1} \leq \cdots \leq L_{K}$. Hence, we will get $K$ intervals $\left[0, L_{1}\right), \ldots,\left(L_{K-1}, L_{K}\right)$.

Function $\mathcal{U}_{m 1}(y)$ is a concave function of $y$ except that at most $K$ non-differentiable points at $\left\{L_{1}, \ldots, L_{K}\right\}$. We then study the function property of $\mathcal{U}_{m 2}(y)$. When $0 \leq y<L_{1}\left(V_{k}=1\right.$ for all $\left.k\right)$, we take the first derivative of $\mathcal{U}_{m 2}(y)$ with respect to $y$, and we have

$$
\frac{\partial \mathcal{U}_{m 2}(y)}{\partial y}=\beta\left(\sum_{k=1}^{K} g_{k m}^{2}\left(\frac{W \mu_{k} x_{r_{k}^{*}} B_{k v}}{\left(\mu_{k} x_{r_{k}^{*}} B_{k v}+\lambda_{k} y g_{k m}^{2}\right)^{2} \ln 2}-\frac{\sigma_{k}^{2}}{h_{k}^{2}}\right)\right)
$$

Since the second derivative of $\mathcal{U}_{m 2}(y)$ with respect to $y$

$$
\frac{\partial^{2} \mathcal{U}_{m 2}(y)}{\partial y^{2}}=-\beta\left(\sum_{k=1}^{K} \frac{2 W \mu_{k} x_{r_{k}^{*}} B_{k v} \lambda_{k} g_{k m}^{4}}{\left(\mu_{k} x_{r_{k}^{*}} B_{k v}+\lambda_{k} y g_{k m}^{2}\right)^{3} \ln 2}\right)<0
$$


$\frac{\partial \mathcal{U}_{m 2}(y)}{\partial y}$ is monotonic decreasing. We know that

$$
\lim _{y \rightarrow 0} \frac{\partial \mathcal{U}_{m 2}(y)}{\partial y}=\beta\left(\sum_{k=1}^{K} g_{k m}^{2}\left(\frac{W}{\mu_{k} x_{r_{k}^{*}} B_{k v} \ln 2}-\frac{\sigma_{k}^{2}}{h_{k}^{2}}\right)\right)>0
$$

When $y \rightarrow L_{1}$, we have two cases: (1) $\frac{\partial \mathcal{u}_{m 2}(y)}{\partial y} \geq 0$. (2) $\frac{\partial \mathcal{u}_{m 2}(y)}{\partial y}<0$. For the first case, $\frac{\partial \mathcal{U}_{m 2}(y)}{\partial y} \geq 0, \mathcal{U}_{m 2}(y)$ is a monotonic increasing function of $y$ at interval $(0, L 1)$. For the second case $\frac{\partial \mathcal{U}_{m 2}(y)}{\partial y}<0, \mathcal{U}_{m 2}(y)$ first increases with $y$, and then decreases with $y$. Therefore, $\mathcal{U}_{m 2}(y)$ is a concave function about $y$ within the interval. The above method can be used for other intervals. Therefore, function $\mathcal{U}_{m 2}(y)$ is a concave function about $y$ except at most $K$ non-differentiable points at $\left\{L_{1}, \ldots, L_{k}\right\}$. Because function $\mathcal{U}_{m 1}(y)$ and function $\mathcal{U}_{m 2}(y)$ are concave functions, function $\mathcal{U}_{m}(y)$ is a concave function except at most $K$ non-differentiable points at $\left\{L_{1}, \ldots, L_{K}\right\}$ [182]. Therefore, $\mathcal{U}_{m}(y)$ could be maximized by solving for $y$. Many optimization methods, such as a binary search algorithm and a gradient based algorithm [182], can be used to solve for $y$ in each interval.

\subsubsection{Analysis of the Homogeneous Bertrand Game for Electricity Re- tailers in Smart Grids}

The retailers use the electricity price as their strategic variable in maximizing the individual profit. The gain of an arbitrary Retailer $r$ depends on not only the electricity price it offers $x_{r}$, and its cost $c_{r}$, but also on the electricity prices offered by other retailers $\mathbf{x}_{-r}$, since the electricity users procure electricity from the retailer with lowest offered price. This price decision problem can be modeled by a homogeneous Bertrand game, since all of the retailers provide electricity, and electricity is a commodity, and so the scenario is homogeneous. The set of the players is $\mathcal{R}=\{1, \ldots, R\}$. 
The strategy set is $x_{r}$, and $\mathcal{U}_{r}$ is the payoff function of Retailer $r$. The Nash equilibrium gives the set of prices such that no retailer can increase its individual net utility by choosing a different price, given the prices offered by the other retailers. Without loss of generality, let that $\operatorname{costs} c_{1}<c_{2} \cdots<c_{R}$. We assume in a Bertrand market, any retailer with zero demand in Nash equilibrium at any price greater or equal to its cost offers a price equal to cost, since the lower price, the better chance of being chosen.

We illustrate the best response function for electricity Retailer1 and Retailer 2 in Fig. 5.13 in the case of a duopoly, where $c_{1}<c_{2}$. The best response of electricity Retailer 1 is the best price offered to maximize its total revenue, given the price offered by the Retailer 2 . In this case, the Nash equilibrium is $\mathbf{x}^{*}=\left(c_{2}-\delta, c_{2}\right)$. The reason is that if Retailer 2 sets its price to $c_{2}$, Retailer 1 has an incentive to lower its price by a small amount $\delta$, and thereby capture the whole market at a price above its cost. Pricing lower than its own cost is not optimal, since it would entail negative profits. This conclusion can extend to multiple retailers, since we assume that the retailer offering the lowest price receives the market's total electricity demand.

Property 3 The Nash equilibrium of the homogeneous Bertrand game with multiple retailers is as follows [183]:

$$
\boldsymbol{x}^{*}=\left\{x_{1}^{*}, c_{2}, c_{3}, \ldots, c_{R}\right\}
$$

where $x_{1}^{*}$ denotes retailer 1 's price strategy at the Nash equilibrium, which can be calculated as follows:

$$
x_{1}^{*}=\arg \max _{c_{1} \leq x_{1}<c_{2}} \mathcal{U}_{1}^{M}\left(x_{1}\right)
$$

where $\mathcal{U}_{1}^{M}\left(x_{1}\right)$ denotes Retailer 1's utility when the retailer supplies the whole market 


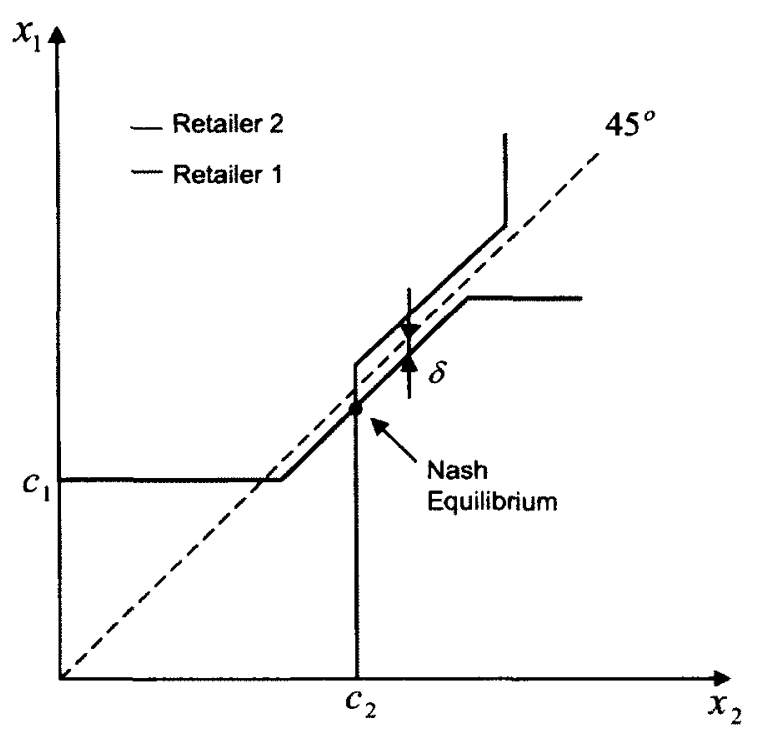

Figure 5.13: Best response functions for homogeneous Bertrand duopoly.

(i.e. monopoly), and it is described as follows:

$$
\begin{aligned}
\mathcal{U}_{1}^{M}\left(x_{1}\right)= & \left(x_{1}-c_{1}\right)\left(P_{m f}+B_{m v}\left[\frac{W}{\alpha x_{1} B_{m v} \ln 2}-\frac{I_{m}}{h_{m}^{2}}\right]^{+}\right. \\
& \left.+\sum_{k=1}^{K}\left(P_{k f}+B_{k v}\left[\frac{W}{\left(\mu_{k} x_{1} B_{k v}+\lambda_{k} y g_{k m}^{2}\right) \ln 2}-\frac{\sigma_{k}^{2}}{h_{k}^{2}}\right]^{+}\right)\right) .
\end{aligned}
$$

Due to the piecewise nature of $\mathcal{U}_{1}^{M}$ with respect to $x_{1}$, we introduce the indicator function:

$$
\begin{aligned}
& D_{k}= \begin{cases}1, & x_{1}<\frac{\frac{W h_{k}^{2}}{\sigma_{k}^{2} \ln 2}-\lambda_{k} y g_{k m}^{2}}{\mu_{k} B_{k v}} \\
0, & \text { otherwise. }\end{cases} \\
& D_{m}= \begin{cases}1, & x_{1}<\frac{W h_{m}^{2}}{\alpha I_{m} B_{m v} \ln 2} \\
0, & \text { otherwise. }\end{cases}
\end{aligned}
$$

Therefore, 


$$
\begin{aligned}
\mathcal{U}_{1}^{M}\left(x_{1}\right)= & \left(x_{1}-c_{1}\right)\left(P_{m f}+B_{m v} D_{m}\left(\frac{W}{\alpha x_{1} B_{m v} \ln 2}-\frac{I_{m}}{h_{m}^{2}}\right)\right. \\
& \left.+\sum_{k=1}^{K}\left(P_{k f}+B_{k v} D_{k}\left(\frac{W}{\left(\mu_{k} x_{1} B_{k v}+\lambda_{k} y g_{k m}^{2}\right) \ln 2}-\frac{\sigma_{k}^{2}}{h_{k}^{2}}\right)\right)\right)
\end{aligned}
$$

We let

$$
\begin{aligned}
E_{k} & =\frac{\frac{W h_{k}^{2}}{\sigma_{k}^{2} \ln 2}-\lambda_{k} y g_{k m}^{2}}{\mu_{k} B_{k v}}, \\
E_{m} & =\frac{W h_{m}^{2}}{\alpha I_{m} B_{m v} \ln 2} .
\end{aligned}
$$

We need to sort all $E_{1}, \ldots, E_{K}$ and $E_{m}$ in ascending order. Without loss generality, let that $E_{1} \leq \cdots \leq E_{k} \leq E_{m}$. Hence, we will have $K+1$ intervals $\left(0, E_{1}\right),\left[E_{1}, E_{2}\right), \ldots,\left[E_{K}, E_{m}\right)$.

When $0<x_{1}<E_{1}$, we take the first derivative of $\mathcal{U}_{1}$ with respect to $x_{1}$ and we have

$$
\begin{aligned}
\frac{\partial \mathcal{U}_{1}}{\partial x_{1}}= & P_{m f}+\sum_{k=1}^{K} P_{k f}-\sum_{k=1}^{K} \frac{B_{k v} \sigma_{k}^{2}}{h_{k}^{2}}-\frac{B_{m v} \sigma_{m}^{2}}{h_{m}^{2}}+\frac{B_{m v}}{h_{m}^{2}}\left(\sum_{k=1}^{K} \frac{g_{k m}^{2} \sigma_{k}^{2}}{h_{k}^{2}}\right) \\
& +\frac{c_{1} W}{\alpha x_{1}^{2} \ln 2}+\sum_{k=1}^{K} \frac{\left(1-\frac{g_{k m}^{2}}{h_{m}^{2}}\right) W B_{k v}\left(\mu_{k} B_{k v} c_{1}+\lambda_{k} y g_{k m}^{2}\right)}{\left(\mu_{k} x_{1} B_{k v}+\lambda_{k} y g_{k m}^{2}\right)^{2} \ln 2} .
\end{aligned}
$$

The second derivative is

$$
\frac{\partial^{2} \mathcal{U}_{1}}{\partial x_{1}^{2}}=-\frac{2 W c_{1}}{\alpha x_{1}^{3} \ln 2}-\sum_{k=1}^{K} \frac{2\left(1-\frac{g_{k m}^{2}}{h_{m}^{2}}\right) \mu_{k} B_{k v}^{2} W\left(c_{1} \mu_{k} B_{k v}+\lambda_{k} y g_{k m}^{2}\right)}{\left(\mu_{k} x_{1} B_{k v}+\lambda_{k} y g_{k m}^{2}\right)^{3} \ln 2}
$$

The first term in (5.76) is less than 0 . When $\frac{g_{k m}^{2}}{h_{m}^{2}}<1$, the second term is less than 0 . This condition can usually be satisfied in the network since FBSs are usually deployed 
indoors, and therefore do not produce significant interference with MUs. Therefore, the above second derivative is less than 0 , which means that $\frac{\partial u_{1}}{\partial x_{1}}$ is monotonic decreasing.

$$
\lim _{x_{1} \rightarrow 0} \frac{\partial \mathcal{U}_{1}\left(x_{1}\right)}{\partial x_{1}}=+\infty>0 .
$$

When $x_{1} \rightarrow E_{1}$, we have two cases: (1) $\frac{\partial \mathcal{u}_{1}\left(x_{1}\right)}{\partial x_{1}} \geq 0$. (2) $\frac{\partial \mathcal{U}_{1}\left(x_{1}\right)}{\partial x_{1}}<0$. For the first case, $\frac{\partial \mathcal{U}_{1}\left(x_{1}\right)}{\partial x_{1}} \geq 0, \mathcal{U}_{1}\left(x_{1}\right)$ is a monotonic increasing function of $x_{1}$ at interval $(0, E 1)$. For

the second case $\frac{\partial \mathcal{U}_{1}\left(x_{1}\right)}{\partial x_{1}}<0, \mathcal{U}_{1}\left(x_{1}\right)$ first increases with $x_{1}$, and then decreases with $x_{1}$. Therefore, $\mathcal{U}_{1}\left(x_{1}\right)$ is a concave function about $x_{1}$ within the interval. Using the same method of analysis on the other intervals, we can obtain that when $x_{1}<E_{m}$, function $\mathcal{U}_{1}\left(x_{1}\right)$ is a concave function about $x_{1}$ except with at most $K+1$ non-differentiable points at $\left\{E_{1}, \ldots, E_{k}, E_{m}\right\}$. Therefore, the Nash equilibrium can be calculated.

\subsubsection{The Proposed Iteration Algorithm to Obtain the Stackelberg Equilibrium}

Given the backward induction to solve the three-stage Stackelberg game in the above subsection, we propose an iteration algorithm as follows to obtain the Stackelberg equilibrium. 
Algorithm 1. An Iteration Algorithm to Obtain the Stackelberg Equilibrium

1. Initialization: For each electricity Retailer $r$, randomly

given an electricity price $x_{r}$

2. Repeat the iteration

(a). The MBS decides on the interference price $y$ and chooses which retailers to procure electricity from and how much electricity to procure

(b). Each FBS performs its energy-efficient power allocation

(c). Retailers update the prices: $x_{r}[t]=\mathcal{B}_{r}\left(\mathbf{x}_{-r}[t-1]\right)$.

(d). Until $\|\mathbf{x}[t]-\mathbf{x}[t-1]\| /\|\mathbf{x}[t-1]\| \leq \varepsilon$

3. End iteration

$\mathcal{B}_{r}()$ is the best response function of Retailer $r$. The best response of electricity Retailer $r$ at iteration $t$ is the best price offered to maximize its total revenue, given the prices offered by the other retailers $\mathbf{x}_{-r}$ at iteration $t-1$. In the proposed algorithm, for each iteration, the MBS can decide its electricity demand and the interference price offered to the FBSs based on the updated electricity prices. The FBSs then performance energy-efficient power allocations. The algorithm will continue until the electricity prices converge. The proposed algorithm can obtain the Stackelberg equilibrium when the prices converge, which will be shown in the simulation section.

In practice, the proposed iteration algorithm to obtain the three-stage Stackelberg equilibrium can be implemented as follows:

1. The electricity retailers in smart grids first randomly offer electricity prices and 
broadcast the information.

2. The MBS receives the price information, and then obtains the channel state information from the FBS and MSUs.

3. The MBS decides on the interference price and which retailer to procure electricity from, and how much electricity to procure

4. Each FBS perform its energy-efficient power allocation.

5. The electricity retailers then update the prices and repeat steps 2,3 and 4 until the prices converge.

\subsubsection{Simulation Results and Discussions}

In this section, we use computer simulations to evaluate the performance of the proposed scheme. In the simulations, we assume that the coverage radius of the macrocell is $1000 \mathrm{~m}$, and the MUs are randomly located in the macrocell. Each femtocell has a coverage radius of $20 \mathrm{~m}$, and is randomly located near the edge of the macrocell. The path loss between FSU and MBS is $15.3+37.6 \log _{10}\left(d_{n m}+L_{o w}\right)$, and the path loss between FBS and FSU is $46.86+20 \log _{10}\left(d_{n}+L_{i w}\right)$, where $d_{n m}$ is the distance between MBS and FSU, $d_{n}$ is the distance between FBS and FSU, the penetration loss of exterior wall $L_{o w}$ and the peneration loss of interior wall $L_{i w}$ are set to $20 \mathrm{~dB}$ and $10 \mathrm{~dB}$, respectively [184]. The small scale fading parameters are the same as those in [185]. The other parameters of the MBS are set as follows: $W=5, P_{d y n}=83 W$, $P_{S t a}=45.5 W, \eta=0.38, N=2[180]$. The other parameters of each FBS are set as follows: $N P_{d y n}+P_{s t a}=8.2 W$ [98]. The general parameters are set as follows: $\mu_{k}=$ $1, \lambda_{k}=1, \alpha=0.5, \beta=10$. We assume that there are 50 sub-channels in each BS.

We first analyze how each FBS's utility is affected by its energy-efficient power 
allocation decision and the interference price offered by the MBS. We also investigate how each FBS makes its power allocation decision based on the interference price offered by the MBS and the lowest price among those offered by the electricity retailers in smart grids. Fig. 5.14 shows that the utility function of the FBS is a concave function, which matches the proof in subsection 5.3.3.1. Therefore, there exists an optimal transmit power for the FBS in order to maximize its net utility. The utility increases with the increase of the transmit power at the beginning due to the corresponding increase of transmission rate. However, when the transmit power reaches a certain level, the utility starts to decrease, since the gain on transmission rate can not balance the electricity cost and interference cost. The figure also shows that a higher interference price leads to a lower utility, since the same level of interference has a higher interference cost. Fig. 5.15 shows that each FBS tries to decrease its interference cost by adaptively adjusting its transmission power to a lower value as the interference price offered by the MBS increases. The figure also shows that for the same interference price, lower optimal electricity price leads to a higher transmission power, due to the lower electricity cost.

We also study how the interference price offered by the MBS affects its utility, and how the interference price combined with the optimal price offered by the electricity retailers in smart grids affects its power allocation strategy. Fig. 5.16 demonstrates that there are two non-differentiable points at $L_{1}$ and $L_{2}$, which matches the proof in Subsection 5.3.3.2. The figure shows that at the beginning, the utility of the MBS is a concave function of the interference price offered by the MBS, and finally reaches a stable level when the interference price is higher than a certain level. The reason for that is because FBSs adjust their transmission powers to zero to avoid the expensive interference cost, when the interference price is too high. The shape of this figure can vary with the values of the parameters. However, in all situations, there exists a sole 


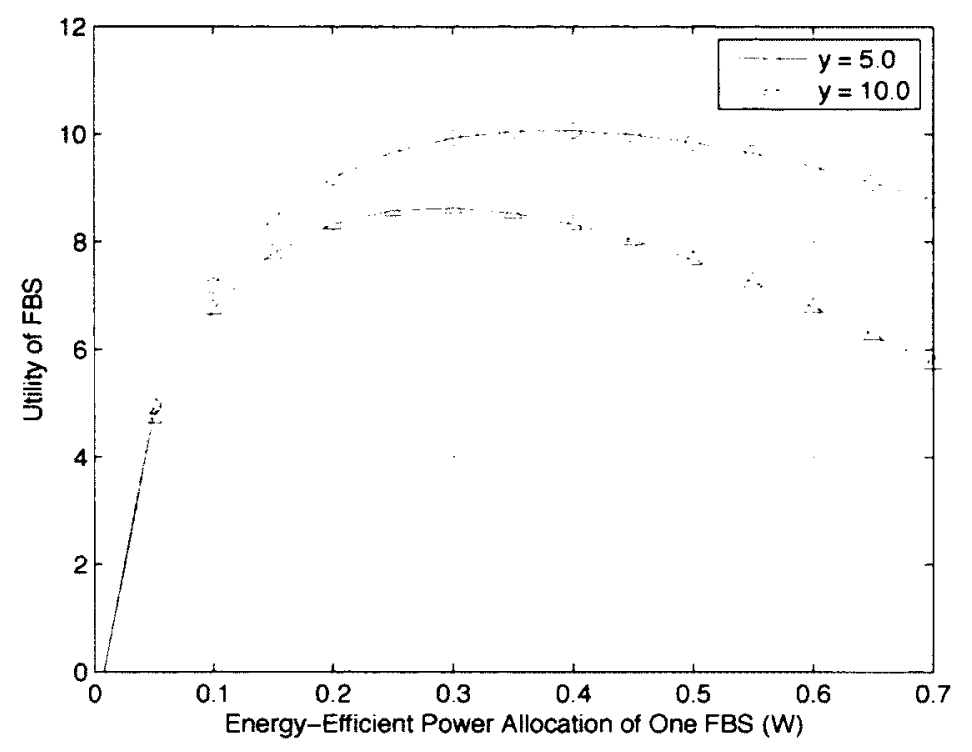

Figure 5.14: Utility function of one FBS versus its power allocation.

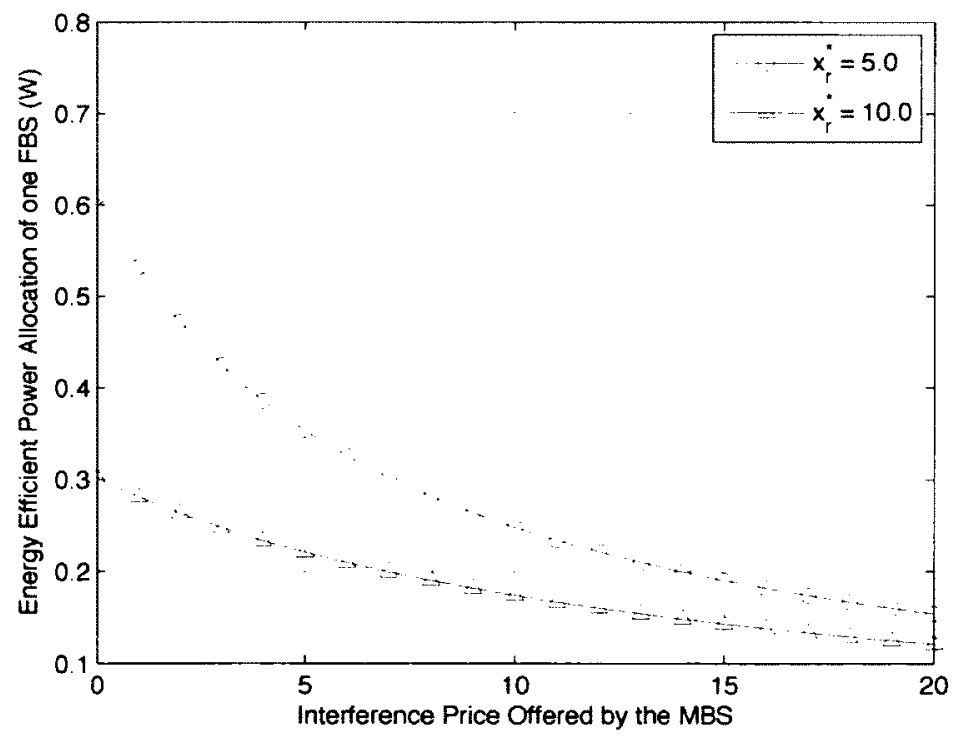

Figure 5.15: Power allocation of the FBS with respect to the interference price offered by the MBS. 


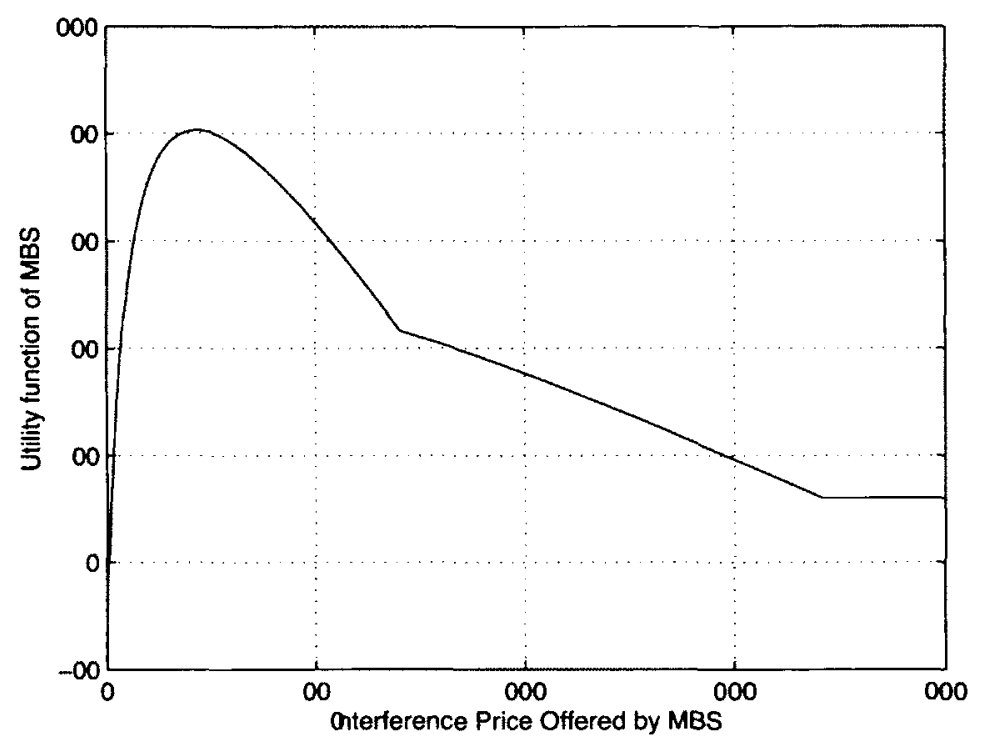

Figure 5.16: Utility function of the MBS with respect to the interference price offered by itself.

optimal interference price in order to maximize the MBS's net utility. Fig 5.17 shows that the MBS's transmit power increases correspondingly with the interference price offered by the MBS, since the interference from the FBSs decreases with the increase of the interference price. The lower optimal electricity price motivates the MBS to increase its transmit power, due to the lower electricity cost.

We analyze how the electricity prices influence the FBSs' and the MBS's strategies in the proposed game. Fig. 5.18 shows that as the lowest price offered by the retailers increases, the base stations decrease their electricity consumption by performing energy-efficient power allocation. The change of the electricity price has a more significant impact on the MBS, since the MBS consumes much more electricity than each FBS does. The exact shape of the curve in the figure changes with the parameters, but this insight remains the same. Fig. 5.19 presents that in a monopoly situation, the utility of the retailer is a concave function with respect to its offered electricity 


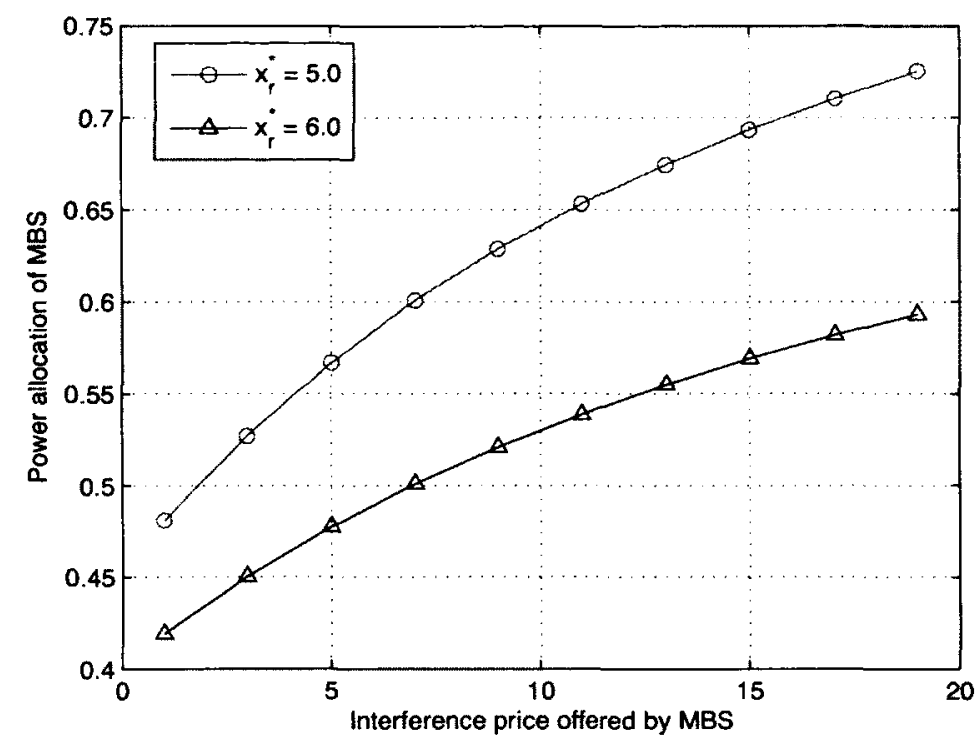

Figure 5.17: Power allocation of the MBS with respect to its interference price.

price, which matches the proof in Subsection 5.3.3.3. The shape of the figure might vary with different sets of parameters. However, there exists a sole optimal electricity price value in all of the situations. The higher the cost, the lower the profit for the electricity retailer, even though the revenue is the same. Fig. 5.20 demonstrates the results using the proposed algorithm to obtain the Stackelberg equilibrium. The figure shows that using the proposed algorithm, the strategies of the FBSs and the MBS can converge after the third iteration due to the convergence of the prices offered by the electricity retailers. Therefore, the Stackelberg equilibrium can be calculated using the proposed algorithm. For the electricity retailers, the Nash equilibrium is that Retailer 2 sets the electricity price to its cost, and the price offered by Retailer 1 is very close to Retailer 2's cost, which matches the proof in Subsection 5.3.3.3.

Finally, we compare the performance of the proposed scheme with the existing scheme proposed in [186] in terms of the total electricity cost and $\mathrm{CO}_{2}$ emissions. In the existing scheme, each BS tries to maximize its capacity under power constraints, 


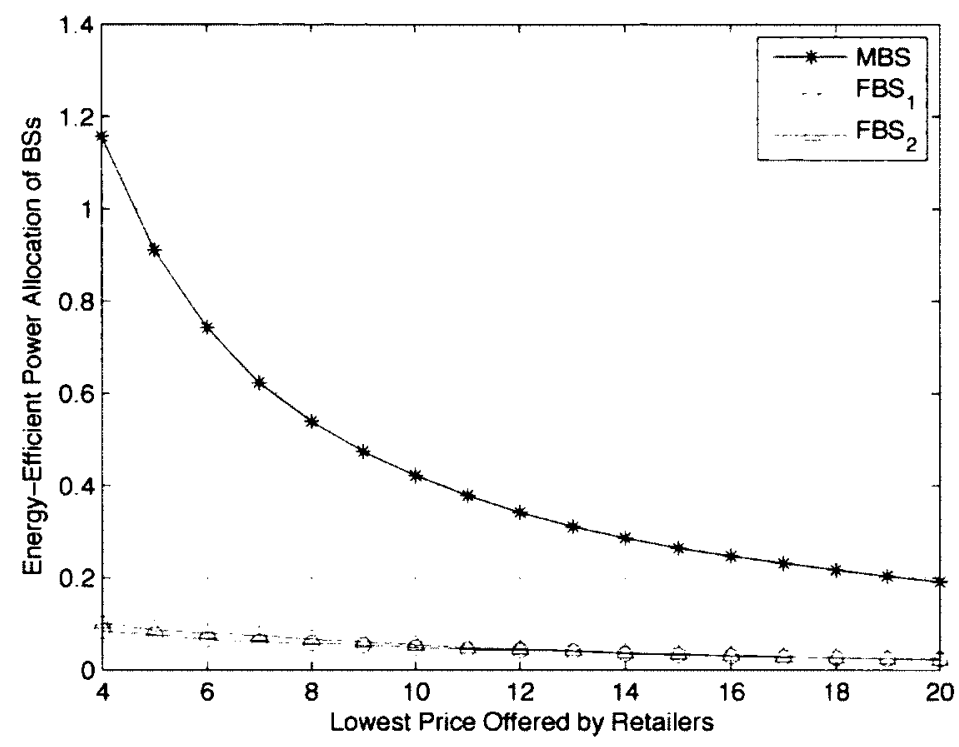

Figure 5.18: Energy-efficient power allocation of the BSs with respect to the lowest prices offered by the retailers.

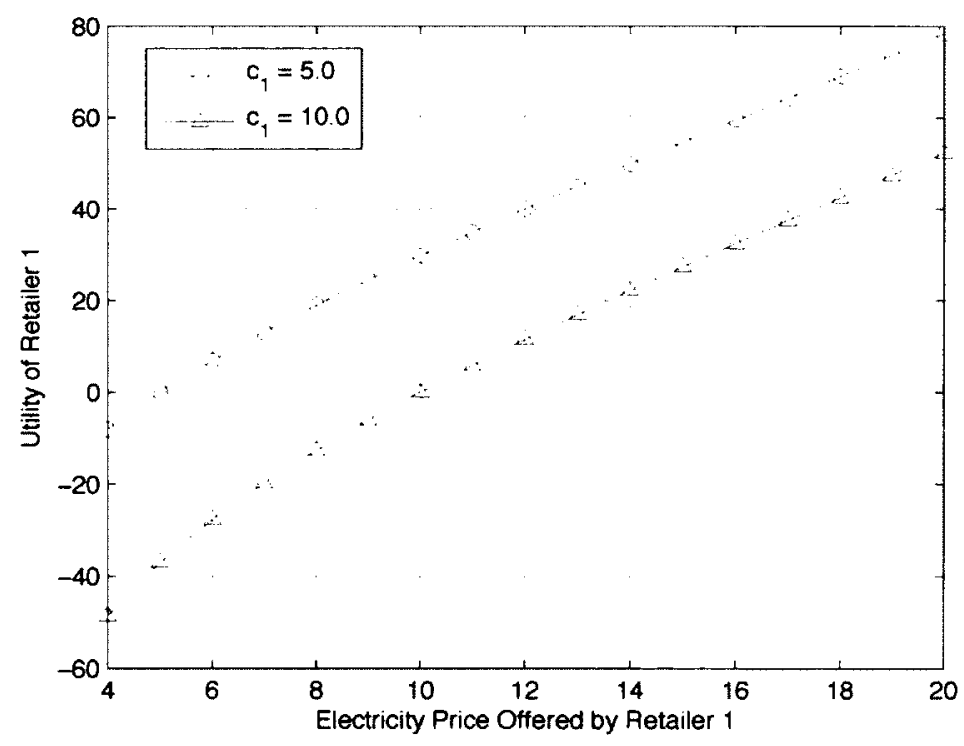

Figure 5.19: Retailer 1's utility in a monoply situation. 

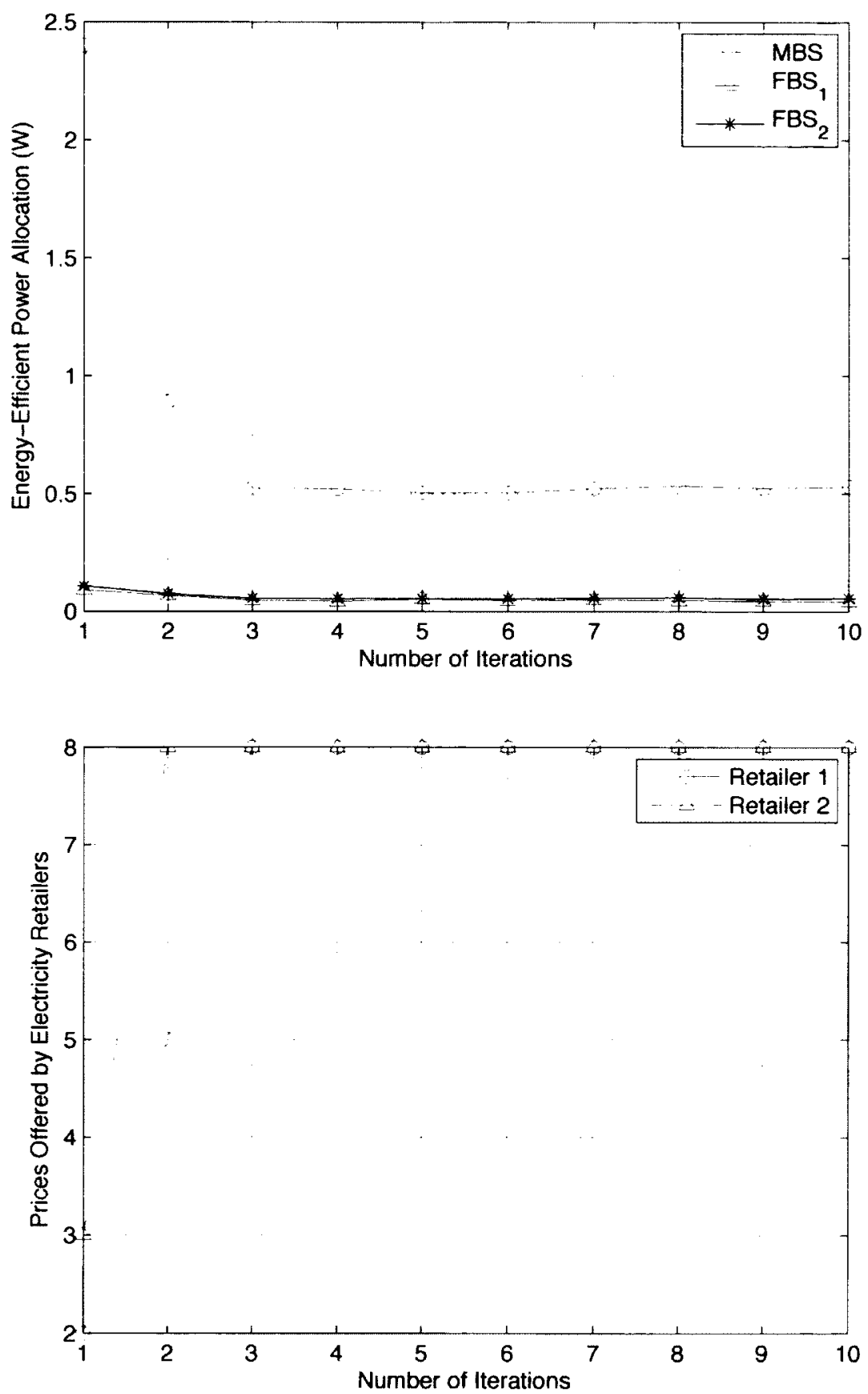

Figure 5.20: Convergence of the Stackelberg equilibrium. 


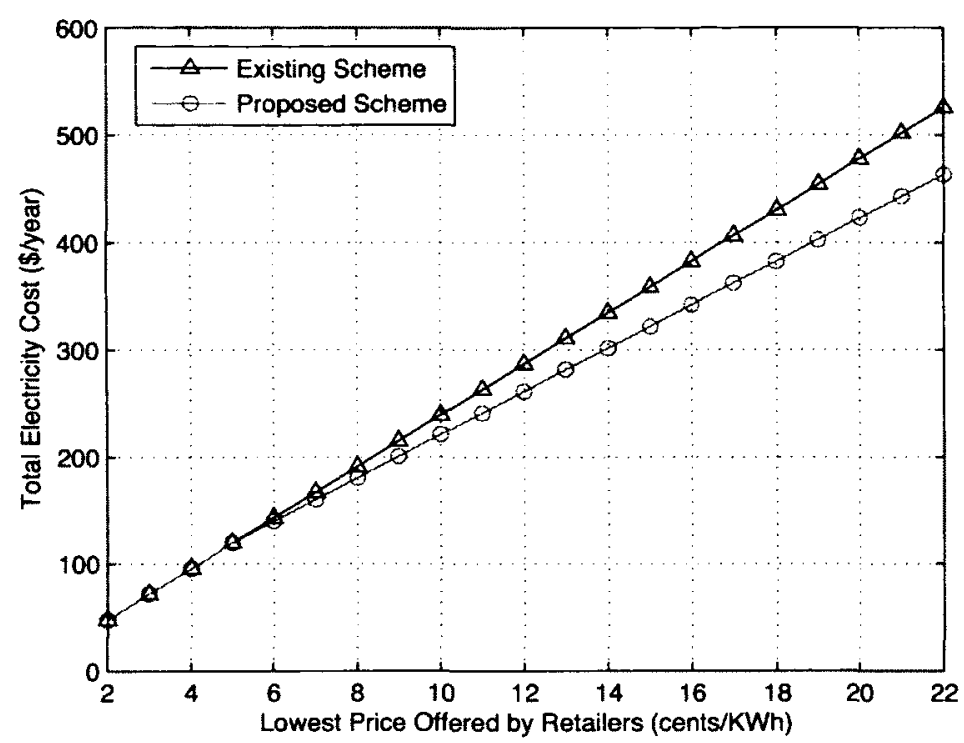

Figure 5.21: Total electricity cost with respect to the lowest price offered by the retailers.

without considering the electricity price. Fig. 5.21 shows that the total electricity cost increases with the lowest price offered by the retailers. In the existing scheme, the BSs produce a constant amount of $\mathrm{CO}_{2}$ emissions with the increasing of the lowest price offered by the retailers, as illustrated in Fig. 5.22. The reason for this is that in the existing scheme, the amount of electricity procured by the BSs does not vary with the electricity price. In the proposed scheme, as the lowest price increases, the BSs decrease their electricity consumption by performing energy-efficient power allocation. Fig. 5.21 and Fig. 5.22 also show that the proposed scheme significantly decreases the total electricity cost and the total $\mathrm{CO}_{2}$ emissions when the electricity price is relatively high. For example, when $x_{r}^{*}=22$ cents $/ k W h$, the proposed scheme leads to a $11.89 \%$ decrease in electricity cost and $\mathrm{CO}_{2}$ emissions compared to the existing scheme. 


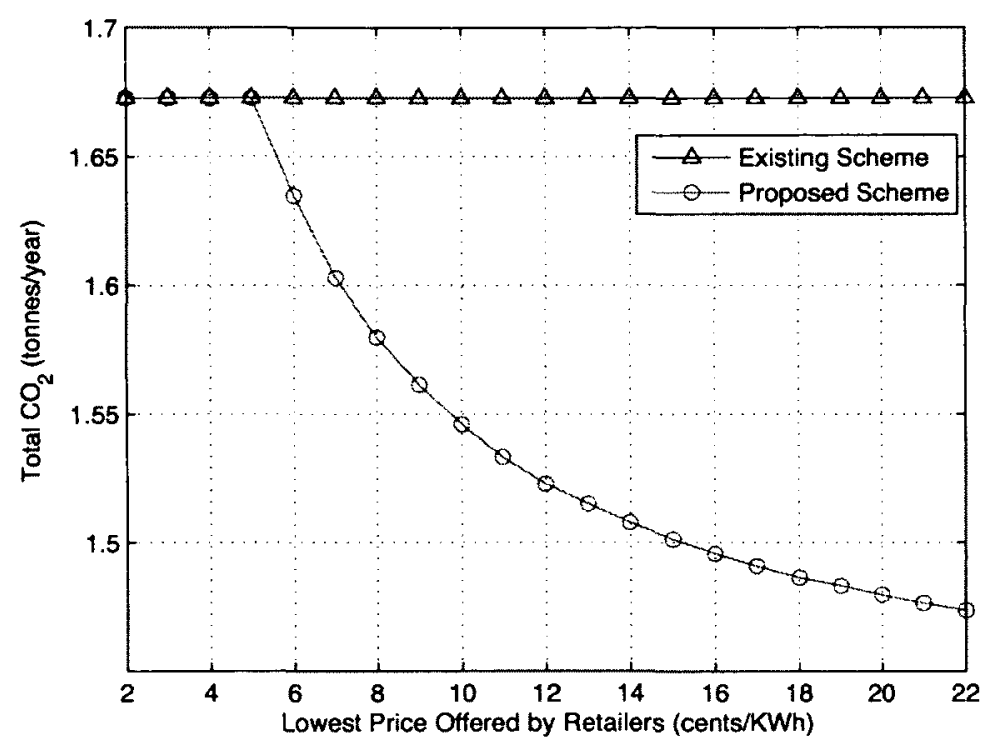

Figure 5.22: Total $\mathrm{CO}_{2}$ emissions with respect to the lowest price offered by the retailers.

\subsection{Conclusion}

In this chapter, we have discussed two important methods of improving the energyefficiency of cellular networks. The first method is the dynamic operation of cellular base stations based on the traffic arrival rate, real-time price provided by smart grids, and pollutant levels of electricity retailers. CoMP is used to extend the coverage of the active BSs, and thus the service quality of mobile users can still be ensured. In the second method, heterogeneous networks, cognitive radios and smart grids are combined to improve the energy-efficiency of the cellular networks. The MBS and FBSs adjust the amount of electricity they consume by performing energy-efficient power allocation. To mitigate the interference effect, and guarantee the QoS, an interference price has been proposed to allow the MBS to protect itself by charging the femtocell users. The system's decision problem has been modeled as a Stackelberg game. A backward induction method was used to analyze the proposed Stackelberg 
game. Simulation results have shown that smart grids have significant impacts on green wireless cellular networks, and the proposed scheme can significantly reduce operational expenditure and $\mathrm{CO}_{2}$ emissions in green wireless cellular networks. 


\section{Chapter 6}

\section{Conclusions and Future Work}

In this chapter, we draw conclusions and discuss possible extensions of the work presented in Chapter 3, Chapter 4 and Chapter 5.

\subsection{Thesis Conclusions}

- A comprehensive survey of high security green mobile ad-hoc networks (MANETs), smart grids and green wireless cellular networks has been provided in Chapter 2. In high security green MANETs, since continuous user authentication and intrusion detection systems (IDSs) have their limitations, jointly considering these two methods can be used to further improve the security performance of the green MANETs. In smart grids, renewable energy resources are intermittent, and power demand loads can be stochastic. Therefore, it is necessary to consider these two characteristics when scheduling power generation systems. In the electricity market, electricity retailers need to make a series of decisions. Therefore, it is necessary to research the full decision process of electricity retailers, since each decision is not independent from the others. Since cellular networks are power hungry elements, smart grids can have a significant impact on energy-efficiency of wireless cellular networks. 
- A distributed scheme combining continuous user authentication and intrusion detection in high security green MANETs has been proposed in Chapter 3 . We used a stochastic optimization method (a partially observable Markov decision process (POMDP) multi-armed bandit problem) to study the scheduling problem considering the security posture and energy states of the nodes. The problem's optimal policy can be chosen using Gittins indices. A value-iteration algorithm and a structural results method were used to calculate the Gittins indices of the nodes. When more than one device was used at each time slot, Dempster-Shafer theory was used for IDS and sensor fusion. The proposed scheme improves network security and network lifetime. Compared to the value iteration algorithm, the structural results method can further reduce computational complexity.

- A distributed stochastic unit commitment (UC) scheduling scheme and a novel game-theoretical decision-making scheme for electricity retailers have been proposed in Chapter 4. In the proposed UC scheduling scheme, the most suitable generation units are dynamically scheduled based on the intermittent renewable energy power generation situations, stochastic power demand loads, utility costs, reliability and pollution emissions of the generation units. Hidden Markov models and a Markov-modulated Poisson process were used in modeling renewable energy resources and the power demand loads, respectively. The UC scheduling problem was studied using a stochastic optimization method (a POMDP multi-armed bandit problem). In the game-theoretical decisionmaking scheme, the interaction between a retailer and its customers has been studied using a stochastic optimization method (a four stage Stackelberg game). Backward induction is used to determine the subgame perfect equilibrium (SPE) of the four-stage Stackelberg game. The proposed UC scheduling scheme can 
decrease the total cost and green gas emissions. The retailer and customers can achieve a higher profit using the proposed decision-making scheme.

- Two important methods of improving the energy-effiency of wireless cellular networks have been discussed in Chapter 5. The first method is the dynamic operation of cellular base stations based on the traffic arrival rate, real-time price provided by smart grids, and pollutant levels of electricity retailers. Coordinated multipoint (CoMP) is used to extend the coverage of the active base stations (BSs), and thus the service quality for the mobile users can still be ensured. In the second method, heterogeneous networks, cognitive radios and smart grids are combined to improve the energy-efficiency of the cellular networks. The macro base station (MBS) and femtocell base stations (FBSs) adjust the amount of electricity they consume by performing energy-efficient power allocation. To mitigate the interference effect, and guarantee the quality of service (QoS), an interference price has been proposed to allow the MBS to protect itself by charging the femtocell users. In these two methods, the system's decision problem has been studied using a stochastic optimization method (a Stackelberg game). A backward induction method was used to analyze the proposed Stackelberg game. The results show that smart grid has a significant impact on energy-efficiency of wireless cellular networks. The proposed scheme can significantly reduce operational expenditure and $\mathrm{CO}_{2}$ emissions in wireless cellular networks.

\subsection{Future Work}

Possible extensions of the work presented in Chapter 3 through Chapter 5 are described as follows: 
1. In the proposed scheme in Chapter 3, only energy posture and security states of the nodes are considered when making the scheduling decisions. Other nodes' state, such as mobility and wireless channels, can be considered in future work, since they have impacts on the scheduling process.

2. In the proposed UC scheduling scheme in Chapter 4 , pricing can be considered in the proposed framework. The impact of pricing on the UC problem can be evaluated and the optimal price assignment strategy can be pursued in future work. In addition, it would be interesting to study the trade-off between system complexity and model accuracy.

3. In the proposed decision-making scheme in Chapter 4 , the interaction between the retailer and the customers has been studied. In our future work, competition among the retailers can be added to the proposed scheme. The scheme can be extended to situations where complete information about customers' utility and preferences cannot be obtained. In such a situation, the system needs to be modeled as a dynamic game with incomplete information.

4. In the proposed scheme in Chapter 5 , we assume that perfect channel state information is known. However, in practice, it is difficult to have perfect knowledge of a dynamic channel. In our future work, we can consider imperfect channel state information in energy-efficient resource allocation for heterogeneous cognitive radio networks. 


\section{List of References}

[1] H. Sistek, "Green-tech base stations cut diesel usage by 80 percent." http: //news. cnet.com/8301-11128_3-9912124-54.html, Apr. 2008.

[2] H. Zhang, A. Gladisch, M. Pickavet, Z. Tao, and W. Mohr, "Energy efficiency in communications," IEEE Communications Magazine, vol. 48, pp. 48-49, Nov. 2010 .

[3] E. Oh, B. Krishnamachari, X. Liu, and Z. Niu, "Toward dynamic energyefficient operation of cellular network infrastructure," IEEE Communications Magazine, vol. 49, pp. 56-61, Jun. 2011.

[4] C. Feisst, D. Schlesinger, and W. Frye, "Smart grid, the role of electricity infrastructure in reducing greenhouse gas emissions," tech. rep., Cisco Internet Business Solution Group, Oct. 2008.

[5] J. Chow, D. Mello, and K. Cheung, "Electricity market design: An integrated approach to reliability assurance," Proceedings of the IEEE, vol. 93, pp. 19561969, Nov. 2005.

[6] M. Parvania and M. Fotuhi-Firuzabad, "Demand response scheduling by stochastic SCUC," IEEE Trans. Smart Grid, vol. 1, pp. 89-98, Jun. 2010.

[7] A. J. Kleywegt and A. Shapiro, "Stochastic optimization," in Handbook of Industrial Engineering (G. Salvendy, ed.), pp. 2625-2650, New York: John Wiley and Sons, 2001.

[8] V. Krishnamurthy and B. Wahlberg, "Partially observed Markov decision process multiarmed bandits - structural results," Math. of Oper. Res., vol. 34, pp. 287-302, May 2009.

[9] F. Y. Ettoumi, H. Sauvageot, and A. Adane, "Statistical bivariate modelling of wind using first-order Markov chain and Weibull distribution," Renewable Energy, vol. 28, pp. 1787-1802, Sept. 2003.

[10] D. Niyato, E. Hossain, and A. Fallahi, "Sleep and wakeup strategies in solarpowered wireless sensor/mesh networks: performance analysis and optimization," IEEE Trans. on Mobile Comp., vol. 6, pp. 221 - 236, Feb. 2007.

[11] A. Daniel and A. A. Chen, "Stochastic simulation and forecasting of hourly average wind speed sequences in Jamaica," Solar Energy, vol. 46, no. 1, pp. 1$11,1991$. 
[12] F. Genoese, M. Genoese, and M. Wietschel, "Occurrence of negative prices on the German spot market for electricity and their influence on balancing power markets," in Proc. International Conference on the European Energy Market, (Madrid, Spain), Jun. 2010.

[13] D. Keles, M. Genoese, D. Most, and W. Fichtner, "Comparison of extended mean-reversion and time series models for electricity spot price simulation considering negative prices," Energy Economics, Aug. 2011. online, doi:10.1016/j.physletb.2003.10.071.

[14] M. Nicolosi, "Wind power integration and power system flexibility - an empirical analysis of extreme events in Germany under the new negative price regime," Energy Policy, vol. 38, pp. 7257-7268, Nov. 2010.

[15] C. Brandstatt, G. Brunekreeft, and K. Jahnke, "How to deal with negative power pricee spikes? - Flexible voluntary curtailment agreements for large-scale integration of wind," Energy Policy, vol. 39, pp. 3732-3740, Jun. 2011.

[16] B. Wang, Y. Wu, and K. J. R. Liu, "Game theory for cognitive radio networks: an overview," Computer Networks, vol. 54, pp. 2537-2561, Oct. 2010.

[17] M. He, S. Murugesan, and J. Zhang, "Multiple timescale dispatch and scheduling for stochastic reliability in smart grids with wind generation integration," in Proc. IEEE INFOCOM, (Shanghai, P.R. China), Apr. 2011.

[18] T. Sim, S. Zhang, R. Janakriaman, and S. Kumar, "Continuous verification using multimodal biometrics," IEEE Trans. Pattern Analysis and Machine Intelligence, vol. 29, pp. 687-700, Apr. 2007.

[19] Q. Xiao, "A biometric authentication approach for high security ad-hoc networks," in Proc. IEEE Info. Assurance Workshop, (West Point, NY), Jun. 2004.

[20] J. Koreman, A. C. Morris, D. Wu, and S. A. Jassim, "Multi-modal biometrics authentication on the secure phone PDA," in Proc. Second Workshop on Multimodal User Authentication, (Toulouse, France), May 2006.

[21] A. Papanikolaou, C. Ilioudis, C. Georgiadis, and E. Pimenidis, "The importance of biometric sensor continuous secure monitoring," in Proc. Third Int'l Conf. on Digital Information Management, (London, UK), Nov. 2008.

[22] J. Muncaster and M. Turk, "Continuous multimodal authentication using dynamic Bayesian networks," in Proc. Second Workshop on Multimodal User Authentication, (Toulouse, France), May 2006.

[23] A. Azzini, S. Marrara, R. Sassi, and F. Scotti, "A fuzzy approach to multimodal biometric continuous authentication," Fuzzy Optim Decis Making, vol. 7, pp. 243-256, Sep. 2008.

[24] A. Altinok and M. Turk, "Temporal integration for continuous multimodal biometrics," in Proc. Workshop Multimodal User Authentication, (Santa Barbara, USA), Dec. 2003. 
[25] A. J. Klosterman and G. R. Ganger, "Secure continuous biometric-enhanced authentication," tech. rep., Carnegie Mellon University Technical Report, May 2000 .

[26] A. Mishra, K. Nadkarni, and V. T. A. Patcha, "Intrusion detection in wireless ad-hoc networks," IEEE Wireless Comm., vol. 11, pp. 48-60, Feb. 2004.

[27] C. Katar, "Combining multiple techniques for intrusion detection," IJCSNS International Journal of Computer Science and Network Security, vol. 6, pp. 208218, Feb. 2006.

[28] S. K. Das, A. Agah, and K. Basu, "Security in wireless mobile and sensor networks," in Wireless Communications Systems and Networks (M. Guizani, ed.), Springer, 2004.

[29] J. Hu, X. Yu, D. Qiu, and H. Chen, "A simple and efficient hidden Markov model scheme for host-based anomaly intrusion detection," IEEE Network, vol. 23, pp. 42-47, Jan. 2009.

[30] G. A. Jacoby and N. J. Davis, "Mobile host-based intrusion detection and attack identification," IEEE Wireless Comm., vol. 14, pp. 53-60, Aug. 2007.

[31] S. N. Chari and P. Cheng, "Bluebox: A policy-driven, host-based intrusion detection system," $A C M$ Trans. on Information and System Security, vol. 6, pp. 173-200, May 2003.

[32] Y. Leu and T. Yang, "A host-based real-time intrusion detection system with data mining and forensic techniques," in Proc. the IEEE 37th Annal Int'l Carnahan Conf. on Security Technology, (Taipei, Taiwan), Oct. 2003.

[33] S. Liu, D. Y. Zhang, X. Chu, H. Otrok, and P. Bhattacharya, "A game theoretic approach to optimize the performance of host-based IDS," in Proc. IEEE international conference on wireless and mobile computing, networking and communication, (Avignon, France), Oct. 2008.

[34] W. Khreich, E. Granger, R. Sabourin, and A. Miri, "Combining hidden Markov models for improved anomaly detection," in Proc. IEEE ICC, (Dresden, Germany), Jun. 2009.

[35] J. Liu, F. Yu, C. H. Lung, and H. Tang, "Optimal combined intrusion detection and biometric-based continuous authentication in high security mobile ad hoc networks," IEEE Trans. Wireless Comm., vol. 8, pp. 806-815, Feb. 2009.

[36] H. Siahkali and M. Vakilian, "Stochastic unit commitment of wind farms integrated in power system," Electric Power Systems Research, vol. 80, pp. 1006 1017, Sept. 2010.

[37] W. Ongsakul and N. Petcharaks, "unit commitment by enhanced adaptive Lagrangian relaxation," IEEE Trans. Power Syst., vol. 19, pp. 620-628, Feb. 2004.

[38] V. S. Pappala and I. Erlich, "A variable-dimension optimization approach to unit commitment problem," IEEE Trans. Power Syst., vol. 25, pp. 1696-1704, Aug. 2010. 
[39] D. N. Simopoulos, S. D. Kavatza, and C. D. Vournas, "Unit commitment by an enhanced simulated annealing algorithm," IEEE Trans. Power Syst., vol. 21, pp. 68-76, Feb. 2006.

[40] C. Y. Chung, H. Yu, and K. P. Wong, "An advance quantum-inspired evolutionary algorithm for unit commitment," IEEE Trans. Power Syst., vol. 26, pp. 847-854, May 2011.

[41] J. Wang, M. Shahidehpour, and Z. Li, "Security-constrained unit commitment with volatile wind power generation," IEEE Trans. Power Syst., vol. 23, pp. 1319-1327, Aug. 2008.

[42] A. Tuohy, P. Meibom, E. Denny, and M. O'Malley, "Unit commitment for systems with significant wind penetration," IEEE Trans. Power Syst., vol. 24, pp. 592-601, May 2009.

[43] V. S. Pappala, I. Erlich, K. Rohrig, and J. Dobschinski, "A stochastic model for the optimal operation of a wind-thermal power system," IEEE Trans. Power Syst., vol. 24, pp. 940-950, May 2009.

[44] L. Xie and M. Ilic, "Model predictive dispatch in electric energy systems with intermittent resources," in Proc. IEEE Int'l Conf. on Systems, Man and Cybernetics, (Singapore), Oct. 2008.

[45] K. Methaprayoon, C. Yingvivatanapong, W. J. Lee, and J. R. Liao, "An integration of ANN wind power estimation into unit commitment considering the forecasting uncertainty," IEEE Trans. Industry Applications, vol. 43, pp. 1441-1448, Nov./Dec. 2007.

[46] E. M. Constantinescu, V. M. Zavala, M. Rocklin, S. Lee, and M. Anitescu, "A computational framework for uncertainty quantification and stochastic optimization in unit commitment with wind power generation," IEEE Trans. Power Syst., vol. 26, pp. 431-441, Feb. 2011.

[47] J. Bialek, P. Varaiya, and F. Wu, "Risk limiting dispatching smart grid," in Talk at CNLS Smart Grid Seminar Series, (Los Alamos, NM), Aug. 2009.

[48] A. D. Sahin and Z. Sen, "First-order Markov chain approach to wind speed modeling," Journal of Wind Engineering and Industrial Aerodynamics, vol. 89, pp. 263-269, Mar. 2001.

[49] P. Poggi, M. Muselli, G. Notton, C. Cristofari, and A. Louche, "Forecasting and simulating wind speed in Corsica by using an autoregressive model," Energy Conversion and Management, vol. 44, pp. 3177-3196, Dec. 2003.

[50] S. Najid, A. Zaharim, A. Razali, M. Zainol, K. Ibrahim, and K. Sopian, "Analyzing the east coast Malaysia wind speed data," International Journal of Energy and Environment, vol. 3, pp. 53-60, 2009.

[51] M. E. Ghitany and N. F. El-Nashar, "Fitting Weibull distribution to ultraviolet solar radiation data," International Journal of Sustainable Energy, vol. 24, pp. 167-173, 2005. 
[52] T. P. Chang, "Investigation on frequency distribution of global radiation using different probability density functions," International Journal of Applied Science and Engineering, vol. 8, pp. 99-107, 2010.

[53] H. S. Bagiorgas, M. Giouli, S. Rehman, and L. M. Al-Hadhrami, "Weibull parameters estimation using four different methods and most energy-carrying wind speed analysis," International Journal of Green Energy, vol. 8, pp. 529554, Aug. 2011.

[54] J. Mur-Amada and A. A. Bayod-Rujula, "Wind power variability model part II - probabilistic power flow," in Proc. 9th International Conference Electrical Power Quality and Utilisation, (Barcelona, Spain), Oct. 2007.

[55] T. Soubdhan, R. Emilion, and R. Calif, "Classification of daily solar radiation distributions using a mixture of Dirichlet distributions," Solar Energy, vol. 83, pp. 1056-1063, Jul. 2009.

[56] G. M. Masters, Renewable and Efficient Electric Power Systems. New York: Wiley, Jul. 2004.

[57] P. Samadi, A. Mohsenian-Rad, R. Schober, V. W. S. Wong, and J. Jatskevich, "Optimal real-time pricing algorithm based on utility maximization for smart grid," in Proc. First IEEE Conf. on Smart Grid Communications, (Gaithersburg, MD), Oct. 2010.

[58] A. H. Mohsenian-Rad and A. Leon-Garcia, "Optimal residential load control with price prediction in real-time electricity pricing environments," IEEE Trans. Smart Grid, vol. 1, pp. 120-133, Sept. 2010.

[59] Hydro Ottawa. http://www.hydroottawa.com. [Online; accessed 05-Aug.2010].

[60] S. Holland and E. Mansur, "Is real-time pricing green? the environmental impacts of electricity demand variance," Rev. Econ. Stat., vol. 90, pp. 550-561, Aug. 2008.

[61] A. H. Mohsenian-Rad, V. W. S. Wong, J. Jatskevich, R. Schober, and A. LeonGarcia, "Autonomous demand-side management based on game-theoretic energy consumption scheduling for the future smart grid," IEEE Trans. Smart Grid, vol. 1, pp. 320-331, Dec. 2010.

[62] G. Gilchrist and et al., "Intelligrid consumer portal telecommunications assessment and specification," tech. rep., Electric Power Research Institute, Palo Alto, CA, Dec. 2005.

[63] Advancing Open Standards for the Information Society (OASIS) Energy Interoperation TC, "Energy interoperation version 1.0," tech. rep., Advancing Open Standards for the Information Society (OASIS), Nov. 2010.

[64] A. Hatami, H. Seif, and M. K. Sheikh-El-Eslami, "A stochastic-based decisionmaking framework for an electricity retailer: time-of-use pricing and electricity protfolio optimization," IEEE Trans. Power Syst., vol. 26, pp. 1808-1816, Nov. 2011. 
[65] A. Eydeland and K. Wolyniec, Energy and Power Risk Management. Wiley, New York, 2003.

[66] C. Triki and A. Violi, "Dynamic pricing of electricity in retailer markets," 4OR, A Quarterly Journal of Operation Research, DOI 10.1007/s10288-007-0056-2.

[67] M. Carrion, A. J. Conejo, and J. M. Arroyo, "Forward contracting and selling price determination for a retailer," IEEE Trans. Power Syst., vol. 22, pp. 21052114, Nov. 2007.

[68] R. G. Karandikar, S. A. Khaparde, and S. V. Kulkarni, "Quantifying price risk of electricity retailer based on CAPM and RAROC methodology," Elec. Power Energy Syst., vol. 29, pp. 803-809, Dec. 2007.

[69] E. Celebi and J. D. Fuller, "A model for efficient consumer pricing schemes in electricity markets," IEEE Trans. Power Syst., vol. 22, pp. 60-67, Feb. 2007.

[70] J. M. Yusta, I. J. Ramirez-Rosado, J. A. Dominguez-Navarro, and J. M. PerezVidal, "Optimal electricity price calculation model for retailers in a deregulated market," International Journal of Electrical Power and Energy Systems, vol. 27, pp. 437-447, Jun.-Jul. 2005.

[71] S. A. Gabriel, M. F. Genc, and S. Balakrishnan, "A simulation approach to balancing annual risk and reward in retail electricity power markets," IEEE Trans. Power Syst., vol. 17, pp. 1050-1057, Nov. 2002.

[72] S. A. Gabriel, A. J. Conejo, M. A. Plazas, and S. Balakrishnan, "Optimal price and quantity determination for retail electric power contracts," IEEE Trans. Power Syst., vol. 21, pp. 180-187, Feb. 2006.

[73] Y. Yan, Y. Qian, H. Sharif, and D. Tipper, "A survey on smart grid communication infrastructures: motivations, requirements and challenges," IEEE Communications Survey $\mathscr{G}$ Tutorials, vol. PP, pp. 1-16, 2012.

[74] D. Niyato, L. Xiao, and P. Wang, "Machine-to-machine communications for home energy management system in smart grid," IEEE Communications Magazine, vol. 49, pp. 53-59, Apr. 2011.

[75] NIST, "NIST framework and roadmap for smart grid interoperability standards, release 1.0," tech. rep. Office of the National Coordinator for Smart Grid Interoperability, Jan. 2010.

[76] Q. Yang, J. A. Barria, and T. C. Green, "Communication infrastructures for distributed control of power distribution networks," IEEE Trans. Ind. Inform., vol. 7, pp. 316-327, May 2011.

[77] K. Moslehi and R. Kumar, "Smart grid - a reliability perspective," in Proc. Invovative Smart Grid Technologies, Jan. 2010.

[78] V. C. Gungor, D. Sahin, T. Kocak, S. Ergut, C. Buccella, C. Cecati, and G. P. Hancke, "Smart grid technologies: communication technologies and standards," IEEE Trans. Ind. Informa., vol. 7, pp. 529-539, Nov. 2011. 
[79] T. Sauter and M. Lobashov, "End-to-end communication architecture for smart grids," IEEE Trans. Ind. Electron., vol. 58, pp. 1218-1228, Apr. 2011.

[80] R. Bo and F. Li, "Probabilistic LMP forecasting considering load uncertainty," IEEE Trans. Power Syst., vol. 24, pp. 1279-1289, Aug. 2009.

[81] G. Bumiller, L. Lampe, and H. Hrasnica, "Power line communications for largescale control and automation systems," IEEE Commun. Mag., vol. 48, pp. 106113, Apr. 2010.

[82] N. Bressan, L. Bazzaco, N. Bui, P. Casari, L. Vangelista, and M. Zorzi, "The deployment of a smart monitoring system using wireless sensors and actuators networks," in Proc. IEEE Int. Conf. Smart Grid Commun., (Gaithersburg, USA), Oct. 2010.

[83] Z. Hasan, H. Boostanimehr, and V. K. Bhargava, "Green cellular networks: a survey, some research issues and challenges," IEEE Communications Surveys \& Tutorials, vol. 13, pp. 524-540, Fourth Quarter 2011.

[84] A. Bianzino, C. Chaudet, D. Rossi, and J. Rougier, "A survey of green networking research," IEEE Communications Surveys \& Tutorials, vol. 14, pp. 3-20, First Quarter 2012.

[85] "Multiradio base station makes network evolution easier and greener than ever." http://www.nokiasiemensnetworks.com/sites/default/files/document/ NokiaSiemensNetworks_2009_02_05_enFlexiMultiradioBTS.pdf, Press Release Feb. 2009, Nokia Siemens Networks. [Online; accessed 02-Feb.-2012].

[86] L. Silu, "The green CDMA base station," Huawei Communicate, pp. $42-43$, Dec. 2008.

[87] M. Ismail and W. Zhuang, "Network cooperation for energy saving in green radio communications," IEEE Wireless Comm., vol. 18, pp. 76-81, Oct. 2011.

[88] Z. Niu, Y. Wu, J. Gong, and Z. Yang, "Cell zooming for cost-efficient green cellular networks," IEEE Communications Magazine, vol. 48, pp. 74-79, Nov. 2010 .

[89] M. A. Marsan, L. Chiaraviglio, D. Ciullo, and M. Meo, "Optimal energy savings in cellular access networks," in Proc. IEEE ICC Workshops, (Dresden, Germany), Jun. 2009.

[90] L. Chiaraviglio, D. Ciullo, M. Meo, and M. A. Marsan, "Energy-aware UMTS access networks," in Proc. International Symposium on Wireless Personal Multimedia Communications, (Lapland, Finland), Sept. 2008.

[91] S. Zhou, J. Gong, Z. Yang, Z. Niu, and P. Yang, "Green mobile access network with dynamic base station energy saving," in Proc. Mobicom Poster, (Beijing, China), Sept. 2009.

[92] A. P. Jardosh, K. Papagiannaki, E. M. Belding, K. C. Almeroth, G. Iannaccone, and B. Vinnakota, "Green WLANs: on-demand WLAN infrastructures," Mobile Networks and Applications, vol. 14, pp. 798-814, Dec. 2009. 
[93] D. Cao, S. Zhou, C. Zhang, and Z. Niu, "Energy saving performance comparison of coordinated multi-point transmission and wireless relaying," in Proc. IEEE GLOBECOM, (Honolulu, USA), Dec. 2010.

[94] G. Gur and F. Alagoz, "Green wireless communications via cognitive dimension: an overview," IEEE Network, vol. 25, pp. 50-56, Mar. 2011.

[95] J. M. III, Cognitive Radio: An Integrated Agent Architecture for Software Defined Radio. PhD thesis, KTH Royal Institute of Technology, 2000.

[96] C. Shannon, "Communication in the presence of noise," Proc. IRE, vol. 37, pp. 10-21, Jan. 1949.

[97] O. Holland, V. Friderikos, and A. Aghvami, "Green spectrum management for mobile operators," in Proc. IEEE GLOBECOM Workshops, (Miami, USA), Dec. 2010.

[98] I. Ashraf, L. T. W. Ho, and H. Claussen, "Improving energy efficiency of femtocell base stations via user activity detection," in Proc. IEEE WCNC, (Sydney, Australia), Apr. 2010.

[99] Y. Hou and D. I. Laurenson, "Energy efficiency of high QoS heterogeneous wireless communication network," in Proc. IEEE VTC Fall, (Ottawa, Canada), Sept. 2010.

[100] T. Zhang and D. H. K. Tsang, "Optimal cooperative sensing scheduling for energy-efficient cognitive radio networks," in Proc. IEEE INFOCOM, (Shanghai, China), Apr. 2011.

[101] Z. Hasan, G. Bansal, E. Hossain, and V. K. Bhargava, "Energy-efficient power allocation in OFDM-based cognitive radio systems: a risk-return model," IEEE Trans. Wireless Comm., vol. 8, pp. 6078-6088, Dec. 2009.

[102] C. H. Ko and H. Y. Wu, "On-demand resource-sharing mechanism design in two-tier ofdma femtocell networks," IEEE Trans. Veh. Tech., vol. 60, pp. 1059 1071, Mar. 2011.

[103] S. Lasaulce, Y. Hayel, R. E. Azouzi, and M. Debbah, "Introducing hierarchy in energy games," IEEE Trans. Wireless Comm., vol. 8, pp. 3833-3843, Jul. 2009.

[104] M. L. Treust and S. Lasaulce, "A repeated game formulation of energy-efficient decentralized power control," IEEE Trans. Wireless Comm., vol. 9, pp. 28602869, Sept. 2010.

[105] S. Buzzi and D. Saturnino, "A game-theoretic approach to energy-efficient power control and receiver design in cognitive cdma wireless networks," IEEE J. Sel. Topics Signal Proc., vol. 5, pp. 137-150, Feb. 2011.

[106] Y. Chen, S. Zhang, S. Xu, and G. Y. Li, "Fundamental trade-offs on green wireless networks," IEEE Communication Magazine, vol. 49, pp. 30-37, Jun. 2011. 
[107] H. Wu, Sensor fusion for context-aware computing using Dempster-Shafer theory. PhD thesis, Carnegie Mellon University, 2003.

[108] S. Bu, F. R. Yu, P. X. Liu, and H. Tang, "Structural results for combined continuous user authentication and intrusion detection in high security mobile ad-hoc networks," IEEE Trans. Wireless Comm., vol. 10, pp. 3064-3073, Sept. 2011.

[109] S. Bu, F. R. Yu, P. X. Liu, , P. Mason, and H. Tang, "Distributed combined authentication and intrusion detection with data fusion in high security mobile ad-hoc networks," IEEE Trans. Veh. Tech., vol. 60, pp. 1025-1036, Mar. 2011.

[110] S. Bu, F. R. Yu, P. X. Liu, and H. Tang, "A computationally efficient method for joint authentication and intrusion detection in mobile ad-hoc networks," in Proc. IEEE ICC, (Kyoto, Japan), Jun. 2011.

[111] S. Bu, F. R. Yu, P. X. Liu, H. Tang, and P. Mason, "Distributed combined authentication and intrusion detection with data fusion in high security mobile ad-hoc networks," in Proc. IEEE Milcom, (San Jose, USA), Oct./ Nov. 2010.

[112] P. Hu, Z. Zhou, Q. Liu, and F. Li, "The HMM-based modeling for the energy level prediction in wireless sensor networks," in Proc. IEEE Conf. on Industrial Electronics and Applications, (Harbin, P.R. China), May 2007.

[113] A. K. Jain, K. Nandakumar, and A. Nagar, "Biometric template security," EURASIP Journal on Advances in Signal Processing, Special Issue on Biometrics, vol. 2008, pp. 1-17, Jan. 2008.

[114] P. Whittle, "Multi-armed bandits and the Gittins index," Journal of Royal Statistical Society Serial B, no. 2, pp. 143-149, 1980.

[115] J. Gittins, Multi-armed Bandit Allocation Indices. Wiley, 1989.

[116] D. Ruta and B. Gabrys, "An overview of classifier fusion methods," Computing and Information Systems, pp. 1-10, 2000.

[117] T. M. Chen and V. Venkataramanan, "Dempster-Shafer theory for intrusion detection in ad hoc networks," IEEE Internet Computing, vol. 9, pp. 35-41, Nov. 2005.

[118] A. R. Cassandra, Exact and approximate algorithms for partially observed Markov decision process. PhD thesis, Brown University, 1998.

[119] V. Krishnamurthy, "Emission management for low probability intercept sensors in network centric warefare," IEEE Trans. Aeros. Elect. Systems, vol. 41, pp. 133-152, Jan. 2005.

[120] A. R. Cassandra. http://www. cassandra.org/pomdp/code/index.shtml/. [Online; accessed 05-Apr.-2010].

[121] Y. Lu, H. Lin, Y. Gu, and A. Helmy, "Towards mobility rich analysis in ad hoc networks: using contraction, expansion and hybrid models," in Proc. IEEE ICC, (Paris, France), Jun. 2004. 
[122] Y. Liang and V. Veeravalli, "Capacity of noncoherent time-selective block Rayleigh flat-fading channel," in Proc. IEEE Symposium of Information Theory, (Lausanne, Switzerland), Jun.-Jul. 2002.

[123] V. Krishnamurthy and D. Djonin, "Structured threshold policies for dynamic sensor scheduling - a partially observed Markov decision process approach," IEEE Trans.Signal Proc., vol. 55, pp. 5069-5083, Oct. 2007.

[124] D. Berstimas and J. Niño-Mora, "Restless bandits, linear programming relaxations, and a primal dual index heuristic," Operations Research, vol. 48, no. 1, pp. $80-90,2000$.

[125] L. Duan, J. Huang, and B. Shou, "Cognitive mobile virtual network operator: investment and pricing with supply uncertainty," in Proc. IEEE INFOCOM, (San Diego, USA), Mar. 2010.

[126] S. Bu, F. R. Yu, and P. X. Liu, "Distributed unit commitment scheduling in the future smart grid with intermittent renewable energy resources and stochastic power demands," under review in International Journal of Green Energy.

[127] S. Bu, F. R. Yu, and P. X. Liu, "A game-theoretical decision-making scheme in the smart grid with demand-side management: towards a smart cyber-physical power infrastructure," under review in IEEE Transactions on Parallel and Distributed System (TPDS) SI on Cyber-Physical Systems.

[128] S. Bu, F. R. Yu, and P. X. Liu, "A game-theoretical decision-making scheme for electricity retailers in the smart grid with demand-side management," in Proc. IEEE SmartGridComm, (Brussels, Belgium), Oct. 2011.

[129] S. Bu, F. R. Yu, and P. X. Liu, "Dynamic pricing for demand-side management in the smart grid," in Proc. IEEE Online Conference on Green Communications, Sept. 2011.

[130] S. Bu, F. R. Yu, P. X. Liu, and P. Zhang, "Distributed stochastic scheduling in smart grid communications with intermittent renewable energy resources," in Proc. IEEE ICC Workshop on Smart Grid Communication, (Kyoto, Japan), Jun. 2011.

[131] S. Bu, F. R. Yu, and P. X. Liu, "Stochastic unit commitment in smart grid communications," in Proc. IEEE INFOCOM Workshop on Green Communications and Networking, (Shanghai, China), Apr. 2011.

[132] R. L. Chen, Models and algorithms for stochastic network design and flow problems: applications in truckload procurement auctions and renewable energy. $\mathrm{PhD}$ thesis, The University of Michigan, 2010.

[133] K. Brokish and J. Kirtley, "Pitfalls of modeling wind power using Markov chains," in Proc. IEEE/PES Power Systems Conf. and Exposition, (Seattle, WA), Mar. 2009.

[134] A. Sayegh, T. D. Todd, and M. Smadi, "Resource allocation and cost in hybrid solar/wind powered WLAN mesh nodes," Wireless Mesh Networks: Architectures and Protocols, pp. 167-189, 2008. 
[135] M. Marwali, S. M. Shahidehpour, and M. Daneshdoost, "Probabilistic production costing for photovoltaics-utility systems with battery storage," IEEE Trans. Energy Conversion, vol. 12, pp. 175-180, Jun. 1997.

[136] W. Fischer and K. Meier-Hellstern, "The Markov-modulated Poisson process (MMPP) cookbook," Performance Evaluation, vol. 18, Sep. 1993.

[137] J. S. Song and P. Zipkin, "Inventories with multiple supply sources and networks of queues with overflow bypasses," tech. rep., Fuqua School of Business, Duke University, Jun. 2007.

[138] C. Lindemann and A. Thummler, "Performance analysis of the general packet radio service," in Proc. 21st Int. Conf. on Distributed Computing Systems (ICDCS), (Phoenix, Arizona), Apr. 2001.

[139] S. M. Ross, Stochastic Processes. John Wiley and Sons, 1983.

[140] L. Wang and C. Singh, "Multicriteria design of hybrid power generation systems based on a modified particle swarm optimization algorithm," IEEE Trans. Energy Conversion, vol. 24, pp. 163-172, Mar. 2009.

[141] B. Bagen, Reliability and cost/worth evaluation of generating systems utilizing wind and solar energy. $\mathrm{PhD}$ thesis, University of Saskatchewan, Aug. 2005.

[142] R. Billinton and R. Allan, Reliability Evaluation of Power Systems. Plenum Press, New York, 1996.

[143] R. D. Smallwood and E. J. Sondik, "The optimal control of partially observable markov processes over a finite horizon," Operations Research, vol. 21.

[144] H. Nfaoui, H. Essiarab, and A. Sayigh, "A stochastic Markov chain model for simulating wind speed time series at Tangiers, Morocco," Renewable Energy, vol. 29, pp. 1407-1418, Jul. 2004.

[145] P. Poggi, G. Notton, M. Muselli, and A. Louche, "Stochastic study of hourly total solar radiation in Corsica using a Markov model," Int'l J. Climatology, vol. 20, pp. 1843-1860, Nov. 2000.

[146] J. S. G. Ehnberg and M. H. J. Bollen, "Simulation of global solar radiation based on cloud observations," Solar Energy, vol. 78, pp. 157-162, Feb. 2005.

[147] M. Erol-Kantarci and H. T. Mouftah, "Using wireless sensor networks for energy-aware homes in smart grids," in Proc. IEEE Sym. on Computers and Commun., (Riccione, Italy), Jun. 2010.

[148] R. H. Liang and J. H. Liao, "A fuzzy-optimization approach for generation scheduling with wind and solar energy systems," IEEE Trans. Power Systems, vol. 22, pp. 1665 - 1674, Nov. 2007.

[149] P. Arun, R. Banerjee, and S. Bandyopadhyay, "Optimum sizing of batteryintegrated diesel generator for remote electrification through design-space approach," Energy, vol. 33, pp. 1155-1168, Jul. 2008. 
[150] M. Fahrioglu, M. Fern, and F. Alvarado, "Designing cost effective demand management contracts using game theory," in Proc. IEEE Power Eng. Soc. Winter Meeting, (New York, NY), Jan. 1999.

[151] T. Jin and M. Mechehoul, "Ordering electricity via internet and its potentials for smart grid systems," IEEE Trans. Smart Grid, vol. 1, pp. 302-310, Dec. 2010.

[152] A. Mas-Colell, M. D. Whinston, and J. R. Green, Microeconomic Theory. Oxford University Press, 1995.

[153] M. J. Osborne, An Introduction to Game Theory. Oxford University Press, Aug. 2003.

[154] B. Wang, Z. Han, and K. J. R. Liu, "Distributed relay selection and power control for multiuser cooperative communication networks using stackelberg game," IEEE Trans. Mobile Comput., vol. 8, pp. 975-990, Jul. 2009.

[155] S. Bu, F. R. Yu, Y. Cai, and P. X. Liu, "When the smart grid meets energyefficient communications: Green wireless cellular networks powered by the smart grid," accepted in IEEE Trans. Wireless Comm., Mar. 2012.

[156] S. Bu, F. R. Yu, and P. X. Liu, "Energy-efficient cognitive heterogeneous wireless networks in the smart grid environment," submitted to IEEE Trans. Veh. Tech., Mar. 2012.

[157] S. Bu, F. R. Yu, Y. Cai, and P. X. Liu, "Energy efficient cellular networks with comp communications and smart grid," in to be presented at ICC GCN, (Ottawa, Canada), Jun. 2012.

[158] S. Bu, F. R. Yu, and P. X. Liu, "Green wireless cellular networks in the smart grid environment," in Green Communications and Networking (F. R. Yu, X. Zhang, and V. C. M. Leung, eds.), CRC Press, 2012.

[159] R. Irmer, H. Droste, P. Marsch, M. Grieger, G. Fettweis, S. Brueck, H.-P. Mayer, L. Thiele, and V. Jungnickel, "Coordinated multipoint: concepts, performance, and field trial results," IEEE Communications Magazine, vol. 49, pp. 102-111, Feb. 2011.

[160] L. Wang and C. Yeh, "3-cell network MIMO architectures with sectorization and fractional frequency reuse," vol. 29, pp. 1185-1199, Jun. 2011.

[161] D. Gesbert, S. Hanly, H. Huang, S. S. Shitz, O. Simeone, and W. Yu, "Multi-cell MIMO cooperative networks: A new look at interference," IEEE J. Sel. Areas Commun., vol. 28, pp. 1380-1408, Dec. 2010.

[162] A. I. Elwalid and D. Mitra, "Effective bandwidth of general Markovian traffic sources and admission control of high speed networks," IEEE/ACM Trans. Netw., vol. 1, pp. 329-343, Jun. 1993.

[163] F. Yu and V. Krishnamurthy, "Effective bandwidth of multimedia traffic in packet wireless CDMA networks with LMMSE receivers - a cross-layer perspective," IEEE Trans. Wireless Comm., vol. 5, pp. 525-530, Mar. 2006. 
[164] L. Kleinrock, Queueing Systems, Volume I: Theory. New York: Wiley Interscience, 1975.

[165] A. J. Fehske, P. Marsch, and G. P. Fettweis, "Bit per joule efficiency of cooperating base stations in cellular networks," in Proc. IEEE GLOBECOM Workshops, (Miami, FL), Dec. 2010.

[166] K. Senthil, "Combined economic emission dispatch using evolutionary programming technique," IJCA Special Issue on Evolutionary Computation for Optimization Techniques, no. 2, pp. 62-66, 2010.

[167] K. Senthil and K. Manikandan, "Improved tabu search algorithm to economic emission dispatch with transmission line constraint," Int'l J. of Computer Science and Comm., vol. 1, pp. 145-149, Jul.-Dec. 2010.

[168] S. M. V. Pandian and et. al., "An efficient particle swarm optimization technique to solve combined economic emission dispatch problem," European Journal of Scientific Research, vol. 54, no. 2, pp. 187-192, 2011.

[169] G. He, S. Lasaulce, and Y. Hayel, "Stackelberg games for energy-efficient power control in wireless networks," in Proc. IEEE INFOCOM Mini-Conference, (Shanghai, China), Apr. 2011.

[170] M. S. Barzaraa, Nonlinear Programming: Theory and Algorithms. John Wiley \& Sons, 1993.

[171] R. Yates, "A framework for uplink power control in cellular radio systems," IEEE J. Sel. Areas Commun., vol. 13, pp. 1341-1347, Sept. 1995.

[172] C. U. Saraydar, N. B. Mandayam, and D. J. Goodman, "Efficient power contro via pricing in wireless data networks," IEEE Trans. Communications, vol. 50, pp. 291-303, Feb. 2002.

[173] R. G. Akl, M. V. Hegde, and M. Naraghi-Pour, "Mobility-based CAC algorithm for arbitrary call-arrival rates in CDMA cellular systems," vol. 54, pp. 639-651, Mar. 2005.

[174] D. Niyato and E. Hossain, "A novel analytical framework for integrated crosslayer study of call-level and packet-level qos in wireless mobile multimedia networks," IEEE Transactions on Mobile Computing, vol. 6, Mar. 2007.

[175] X. Kang, Y. Liang, and H. K. Garg, "Distributed power control for spectrumsharing femtocell networks using Stackelberg game," in Proc. IEEE ICC, (Kyoto, Japan), Jun. 2011.

[176] V. Chandrasekhar, J. G. Andrews, T. Muharemovic, Z. Shen, and A. Gatherer, "Power control in two-tier femtocell networks," IEEE Trans. Wireless Comm., vol. 8, pp. 4316-4328, Aug. 2009.

[177] X. Kang, R. Zhang, and M. Motani, "Price-based resource allocation for spectrum-sharing femtocell networks: A Stackelberg game approach," IEEE JSAC Special Issue on Femtocell Networks, Apr. 2012. 
[178] S. Verdu, "Spectral efficiency in the wideband regime," IEEE Trans. Inf. Theory, vol. 48, pp. 1319-1343, Jun. 2002.

[179] G. Miao, N. Himayat, G. Y. Li, A. T. Koc, and S. Talwar, "Interference-aware energy-efficient power optimization," in Proc. IEEE ICC, (Dresden, Germany), Jun. 2009.

[180] J. Xu, L. Qiu, and C. Yu, "Improving energy efficiency through multimode transmission in the downlink MIMO systems," EURASIP Journal on Wireless Communication and Networking, vol. 2011, pp. 1-12, Dec. 2011.

[181] D. P. Palomar and M. Chiang, "A tutorial on decomposition methods for network utility maximization," IEEE J. Sel. Areas Commun., vol. 24, pp. 1439 1451, Aug. 2006.

[182] S. Boyd and L. Vandenberghe, Convex Optimization. Cambridge University Press, 2004.

[183] A. Ledvina and R. Sircar, "Static and dynamic oligopoly games under asymmetric costs," submitted, 2011.

[184] S. Y. Lien, Y. Y. Lin, and K. C. Chen, "Cognitive and game-theoretical radio resource management for autonomous femtocells with qos guarantees," IEEE Trans. Wireless Comm., vol. 10, pp. 2196-2206, Jul. 2011.

[185] 3rd Generation Partnership Project, "Further advancements for e-utra physical layer aspects," Tech. Rep. 3GPP TS 36.814 V9.0.0, Oct. 2010.

[186] S. Guruacharya, D. Niyato, E. Hossain, and D. I. Kim, "Hierarchical competition in femtocell-based cellular networks," in Proc. IEEE GLOBECOM, (Miami, USA), Dec. 2010. 